HEISENBERG ALGEBRAS

\title{
AND RATIONAL DOUBLE AFFINE HECKE ALGEBRAS
}

\author{
P. SHAN AND E. VASSEROT
}

\section{Contents}

1. Introduction and notation

1.1. Introduction

1.2. Organisation

1.3. Notation

2. Reminder on rational DAHA's

2.1. The category $\mathcal{O}(W)$

2.2. The stratification of $\mathfrak{h}$

2.3. Induction and restriction functors on $\mathcal{O}(W)$

3. The cyclotomic rational DAHA

3.1. Combinatorics

3.2. The complex reflection group $\Gamma_{n}$

3.3. Definition of the cyclotomic rational DAHA

3.4. The Lie algebras $\widehat{\hat{\mathfrak{s l}}_{\ell}}$ and $\widetilde{\mathfrak{s l}}_{\ell}$

3.5. Representations of $\mathfrak{S}_{n}, \Gamma_{n}$

3.6. The category $\mathcal{O}\left(\Gamma_{n}\right)$

3.7. The functor $\mathrm{KZ}$

3.8. The functor $R$

3.9. The categorification of $\widetilde{\mathfrak{s l}}_{m}$

3.10. The filtration of $\left[\mathcal{O}\left(\Gamma_{n}\right)\right]$ by the support

3.11. The action of $E, F$ on the filtration

4. The Fock space

4.1. The Hopf $\mathbb{C}$-algebra $\lambda$

4.2. The Heisenberg algebra

4.3. The Lie algebras $\widehat{\mathfrak{g l}}_{m}$ and $\widetilde{\mathfrak{g l}}_{m}$

4.4. The Hopf $\mathbb{C}$-algebra $\lambda_{\Gamma}$

4.5. The level 1 Fock space

4.6. The level $\ell$ Fock space

5. The categorification of the Heisenberg algebra

5.1. The functors $A_{\lambda, !}, A_{\lambda}^{*}, A_{\lambda, *}$ on $D^{b}(\mathcal{O}(\Gamma))$

5.2. The $\mathfrak{S}_{r}$-action on $\left(A_{!}\right)^{r},\left(A^{*}\right)^{r}$ and $\left(A_{*}\right)^{r}$.

5.3. The functors $a_{\lambda}^{*}, a_{\lambda, *}$ on $\mathcal{O}(\Gamma)$ and the $\mathfrak{H}$-action on the Fock space

5.4. Primitive modules

5.5. Endomorphisms of induced modules

5.6. Definition of the map $\tilde{a}_{\lambda}$

Received by the editors March 22, 2011 and, in revised form, October 30, 2011, and February 12, 2012.

2010 Mathematics Subject Classification. Primary 06B15, 33D80. 
6. The filtration of the Fock space and Etingof's conjecture 1004

6.1. The representation-theoretic interpretation of $F_{0,0}(\Gamma) \quad 1005$

6.2. The representation-theoretic grading on $[\mathcal{O}(\Gamma)] \quad 1006$

6.3. Etingof's conjecture 1008

6.4. Reminder on the level-rank duality 1009

6.5. Comparison of the $\tilde{\mathfrak{g l}}_{\ell}$-modules $\mathcal{F}_{m, \ell}^{(d)}$ and $V_{\omega_{d \bmod \ell}}^{\tilde{\mathfrak{g l}}_{\ell}} 1010$

6.6. Proof of Theorem 6.10 1011

Appendix A. Reminder on Hecke algebras 1017

A.1. Affine Hecke algebras 1017

A.2. Cyclotomic Hecke algebras 1018

A.3. Induction/restriction for cyclotomic Hecke algebras 1019

Appendix B. Reminder on $\zeta$-Schur algebras 1021

B.1. The quantized modified algebra 1021

B.2. The $\zeta$-Schur algebra $\quad 1023$

B.3. The module category of $S_{\zeta}(n, m) \quad 1024$

B.4. The Schur functor 1024

B.5. The braiding and the Schur functor 1026

B.6. The braiding and the quantum Frobenius homomorphism 1027

B.7. The algebra $S_{\zeta}(m) \quad 1028$

Appendix C. Remarks on the Fock space 1028

\begin{tabular}{ll} 
Index of notation & 1029 \\
\hline
\end{tabular}

Acknowledgments $\quad 1030$

References $\quad 1030$

\section{INTRODUCTION AND NOTATION}

1.1. Introduction. In this paper we study a relationship between the representation theory of certain rational double affine Hecke algebras (= RDAHA) and the representation theory of affine Kac-Moody algebras. Such a connection is not new and has already appeared in several places in the literature. A first place is Suzuki's functor [34] which maps the Kazhdan-Lusztig category of modules over the affine Kac-Moody algebra $\widehat{\mathfrak{s l}}_{n}$ at a negative level to the representation category of the RDAHA of $\mathfrak{s l}_{m}$. A second one is a cyclotomic version of Suzuki's functor [36] which maps a more general version of the parabolic category $\mathcal{O}$ of $\widehat{\mathfrak{s l}}_{n}$ at a negative level to the representation category of the cyclotomic RDAHA. A third one comes from the relationship between the cyclotomic RDAHA and quiver varieties, see, e.g., [15, and from the relationship between quiver varieties and affine Kac-Moody algebras. Finally, a fourth one, which is closer to our study, comes from the relationship in [33. between the Grothendieck group of cyclotomic RDAHA and the level $\ell$ Fock space $\mathcal{F}_{m, \ell}$ of $\widehat{\mathfrak{s l}}_{m}$. In this paper we focus on a recent conjecture of Etingof [11] which relates the support of the objects of the category $\mathcal{O}$ of $H\left(\Gamma_{n}\right)$, the RDAHA associated with the complex reflection group $\Gamma_{n}=\mathfrak{S}_{n} \ltimes\left(\mathbb{Z}_{\ell}\right)^{n}$, to a representationtheoretic grading of the Fock space $\mathcal{F}_{\ell}=\mathcal{F}_{\ell, 1}$. This grading is constructed using the $\widehat{\mathfrak{g l}}_{\ell}$-action on $\mathcal{F}_{\ell}$ of level 1. These conjectures permit us in particular to compute the number of finite-dimensional $H\left(\Gamma_{n}\right)$-modules. This was not known so far. The appearance of the Fock space $\mathcal{F}_{\ell}$ is due to the following two facts, already noticed 
in [11. First, the category $\mathcal{O}$ of the algebras $H\left(\Gamma_{n}\right)$ with $n \geqslant 0$ categorifies $\mathcal{F}_{m, \ell}$ by 33 . Next, the Fock space $\mathcal{F}_{m, \ell}$ admits a level 1 action of $\widehat{\mathfrak{g l}}_{\ell}$, under which it is identified with $\mathcal{F}_{\ell}$. It is well known that the Fock space $\mathcal{F}_{m, \ell}$ admits an action of a Heisenberg algebra $\mathfrak{H}$ of level $m \ell$, an action of $\widehat{\mathfrak{s l}}_{\ell}$ of level $m$ and an action of $\widehat{\mathfrak{s l}}_{m}$ of level $\ell$ which commute with each other, by the level-rank duality. Our proof consists precisely of interpreting the support of the $H\left(\Gamma_{n}\right)$-modules in terms of the actions of $\widehat{\mathfrak{s l}}_{m}$ and $\mathfrak{H}$ on $\mathcal{F}_{m, \ell}$. Then, we interpret this construction in terms of the $\widehat{\mathfrak{g l}}_{\ell^{-}}$-action on $\mathcal{F}_{\ell}$ of level 1. An important new ingredient is a categorification (in a weak sense) of the action of the Heisenberg algebra $\mathfrak{H}$ on $\mathcal{F}_{m, \ell}$. The categorification of the Heisenberg algebra has recently been studied by several authors. We'll come back to this in another publication.

1.2. Organisation. The organisation of the paper is the following.

Section 2 is a reminder on rational DAHA. We recall some basic facts concerning parabolic induction/restriction functors. In particular we describe their behavior on the support of the modules.

Section 3 contains basic notation for complex reflection groups, for the cyclotomic rational DAHA $H\left(\Gamma_{n}\right)$ and for affine Lie algebras. In particular we introduce the category $\mathcal{O}\left(\Gamma_{n}\right)$ of $H\left(\Gamma_{n}\right)$-modules, the functor $\mathrm{KZ}$, and Rouquier's equivalence from $\mathcal{O}\left(\mathfrak{S}_{n}\right)$ to the module category of the $\zeta$-Schur algebra. Next we recall the categorification of the Fock space representation of $\widehat{\mathfrak{s l}}_{m}$ in 33 , and we describe the filtration by the support on $\mathcal{O}\left(\Gamma_{n}\right)$.

Section 4 is more combinatorial. We recall several constructions related to Fock spaces and symmetric polynomials. In particular we give a relation between symmetric polynomials and the representation ring of the group $\Gamma_{n}$, and we describe several representations on the level $\ell$ Fock space (of Heisenberg algebras and of affine Kac-Moody algebras).

Section 5 is devoted to the categorification of the Heisenberg action on the Fock space, using $\mathcal{O}\left(\Gamma_{n}\right)$. Then we introduce a particular class of simple objects in $\mathcal{O}\left(\Gamma_{n}\right)$, called the primitive modules, and we compute the endomorphism algebra of some modules induced from primitive modules. Finally we introduce the operators $\tilde{a}_{\lambda}$ which are analogues for the Heisenberg algebra of the Kashiwara operators $\tilde{e}_{q}, \tilde{f}_{q}$ associated with Kac-Moody algebras.

Section 6 contains the main results of the paper. Using our previous constructions we compare the filtration by the support on $\mathcal{O}\left(\Gamma_{n}\right)$ with a representationtheoretic grading on the Fock space. This confirms a conjecture of Etingof and yields, in particular, the number of finite-dimensional simple objects in $\mathcal{O}\left(\Gamma_{n}\right)$ for integral $\ell$-charge (this corresponds to some rational values of the parameters of $\left.H\left(\Gamma_{n}\right)\right)$.

Finally, there are three appendices containing basic facts on Hecke algebras, Schur algebras, quantum groups, quantum Frobenius homomorphism and on the universal R-matrix.

1.3. Notation. Now we introduce some general notation. Let $\mathcal{A}$ be a $\mathbb{C}$-category, i.e., a $\mathbb{C}$-linear additive category. We'll write $Z(\mathcal{A})$ for the center of $\mathcal{A}$, a $\mathbb{C}$-algebra. Let $\operatorname{Irr}(\mathcal{A})$ be the set of isomorphism classes of simple objects of $\mathcal{A}$. If $\mathcal{A}=\operatorname{Rep}(\mathbf{A})$, the category of all finite-dimensional representations of a $\mathbb{C}$-algebra $\mathbf{A}$, we abbreviate

$$
\operatorname{Irr}(\mathbf{A})=\operatorname{Irr}(\operatorname{Rep}(\mathbf{A})) .
$$


For an Abelian or triangulated category let $K(\mathcal{A})$ denote its Grothendieck group. We abbreviate $K(\mathbf{A})=K(\operatorname{Rep}(\mathbf{A}))$. We set

$$
[\mathcal{A}]=K(\mathcal{A}) \otimes \mathbb{C} .
$$

For an object $M$ of $\mathcal{A}$ we write $[M]$ for the class of $M$ in $[\mathcal{A}]$. For an Abelian category $\mathcal{A}$ let $D^{b}(\mathcal{A})$ denote its bounded derived category. We abbreviate $D^{b}(\mathbf{A})=$ $D^{b}(\operatorname{Rep}(\mathbf{A}))$. The symbol " $m$ " will denote both the complex

$$
\langle m\rangle=\bigoplus_{i=0}^{m-1} \mathbb{C}[-2 i] \in D^{b}(\mathbb{C})
$$

and the integer $m$ in $K(\mathbb{C})=\mathbb{Z}$. Given two Abelian $\mathbb{C}$-categories $\mathcal{A}, \mathcal{B}$ which are Artinian (i.e., objects are of finite length and Hom's are finite dimensional) we define the tensor product (over $\mathbb{C}$ )

$$
\otimes: \mathcal{A} \times \mathcal{B} \rightarrow \mathcal{A} \otimes \mathcal{B}
$$

as in [6, sec. 5.1, prop. 5.13]. Recall that for $\mathcal{A}=\operatorname{Rep}(\mathbf{A})$ and $\mathcal{B}=\operatorname{Rep}(\mathbf{B})$ we have $\mathcal{A} \otimes \mathcal{B}=\operatorname{Rep}(\mathbf{A} \otimes \mathbf{B})$. Given a category $\mathcal{A}$ and objects $A, A^{\prime} \in \mathcal{A}$, we write $\operatorname{Hom}_{\mathcal{A}}\left(A, A^{\prime}\right)$ for the collection of morphisms $A \rightarrow A^{\prime}$. Given categories $\mathcal{A}, \mathcal{B}$ and functors $F, F^{\prime}: \mathcal{A} \rightarrow \mathcal{B}$ we write $\operatorname{Hom}\left(F, F^{\prime}\right)$ for the collection of morphisms $F \rightarrow F^{\prime}$. We denote the identity morphism $A \rightarrow A$ by $\mathbf{1}_{A}$ and the identity morphism $F \rightarrow F$ by $\mathbf{1}_{F}$. Given a category $\mathcal{C}$ and a functor $G: \mathcal{B} \rightarrow \mathcal{C}$ let $G \circ F$ be the composed functor $\mathcal{A} \rightarrow \mathcal{C}$. For a functor $G^{\prime}: \mathcal{B} \rightarrow \mathcal{C}$ and morphisms of functors $\phi \in \operatorname{Hom}\left(F, F^{\prime}\right), \psi \in \operatorname{Hom}\left(G, G^{\prime}\right)$ we write $\psi \phi$ for the morphism of functors $G \circ F \rightarrow G^{\prime} \circ F^{\prime}$ given by

$$
(\psi \phi)(A)=\psi\left(F^{\prime}(A)\right) \circ G(\phi(A)) \in \operatorname{Hom}_{\mathcal{C}}\left(G(F(A)), G^{\prime}\left(F^{\prime}(A)\right)\right), \quad A \in \mathcal{A} .
$$

\section{REMinder ON RATIONAL DAHA'S}

2.1. The category $\mathcal{O}(W)$. Let $W$ be any complex reflection group. Let $\mathfrak{h}$ be the reflection representation of $W$. Let $S$ be the set of pseudo-reflections in $W$. Let $c: S \rightarrow \mathbb{C}$ be a map that is constant on the $W$-conjugacy classes. The rational $D A H A$ attached to $W$ with parameter $c$ is the quotient $H(W)$ of the smash product of $\mathbb{C} W$ and the tensor algebra of $\mathfrak{h} \oplus \mathfrak{h}^{*}$ by the relations

$$
\left[x, x^{\prime}\right]=0, \quad\left[y, y^{\prime}\right]=0, \quad[y, x]=\langle x, y\rangle-\sum_{s \in S} c_{s}\left\langle\alpha_{s}, y\right\rangle\left\langle x, \check{\alpha}_{s}\right\rangle s,
$$

for all $x, x^{\prime} \in \mathfrak{h}^{*}, y, y^{\prime} \in \mathfrak{h}$. Here $\langle\bullet, \bullet\rangle$ is the canonical pairing between $\mathfrak{h}^{*}$ and $\mathfrak{h}$, the element $\alpha_{s}$ is a generator of $\operatorname{Im}\left(\left.s\right|_{\mathfrak{h}^{*}}-1\right)$ and $\check{\alpha}_{s}$ is the generator of $\operatorname{Im}\left(\left.s\right|_{\mathfrak{h}}-1\right)$ such that $\left\langle\alpha_{s}, \check{\alpha}_{s}\right\rangle=2$. Let $R_{x}, R_{y}$ be the subalgebras generated by $\mathfrak{h}^{*}$ and $\mathfrak{h}$ respectively. We may abbreviate

$$
\mathbb{C}[\mathfrak{h}]=R_{x}, \quad \mathbb{C}\left[\mathfrak{h}^{*}\right]=R_{y} .
$$

The category $\mathcal{O}$ of $H(W)$ is the full subcategory $\mathcal{O}(W)$ of the category of $H(W)$ modules consisting of objects that are finitely generated as $\mathbb{C}[\mathfrak{h}]$-modules and $\mathfrak{h}$ locally nilpotent. We recall from [14, sec. 3] the following properties of $\mathcal{O}(W)$. It is a quasi-hereditary category. The standard modules are labeled by the set $\operatorname{Irr}(\mathbb{C} W)$ of isomorphism classes of irreducible $W$-modules. Let $\Delta_{\chi}$ be the standard module associated with the module $\chi \in \operatorname{Irr}(\mathbb{C} W)$. It is the induced module

$$
\Delta_{\chi}=\operatorname{Ind}_{W \ltimes R_{y}}^{H(W)}(\chi) .
$$


Here $\chi$ is regarded as a $W \ltimes R_{y}$-module such that $\mathfrak{h}^{*} \subset R_{y}$ acts by zero. Let $L_{\chi}$, $P_{\chi}$ denote the top and the projective cover of $\Delta_{\chi}$.

Remark 2.1. The definitions above still make sense if $\mathfrak{h}$ is any faithful finitedimensional $\mathbb{C} W$-module. To avoid any confusion we may write

$$
\mathcal{O}(W, \mathfrak{h})=\mathcal{O}(W), \quad H(W, \mathfrak{h})=H(W) .
$$

2.2. The stratification of $\mathfrak{h}$. Let $W$ be a complex reflection group. Let $\mathfrak{h}$ be the reflection representation of $W$. For a parabolic subgroup $W^{\prime} \subset W$ let $X_{W^{\prime}}^{\circ}$ be the set of points of $\mathfrak{h}$ whose stabilizer in $W$ is conjugate (in $W$ ) to $W^{\prime}$. By a theorem of Steinberg, the sets $X_{W^{\prime}}^{\circ}$, when $W^{\prime}$ runs over a set of representatives of the $W$ conjugacy classes of parabolic subgroups of $W$, form a stratification of $\mathfrak{h}$ by smooth locally closed subsets; see also [13, sec. 6] and the references there. Let $X_{W^{\prime}}$ be the closure of $X_{W^{\prime}}^{\circ}$ in $\mathfrak{h}$. To avoid any confusion we may write $X_{W^{\prime}, \mathfrak{h}}^{\circ}=X_{W^{\prime}}^{\circ}$ and $X_{W^{\prime}, \mathfrak{h}}=X_{W^{\prime}}$. The set $X_{W^{\prime}, \mathfrak{h}}$ consists of points whose $W$-conjugacy class contains a fixed point of $W^{\prime}$ in $\mathfrak{h}$. We have

$$
X_{W^{\prime}, \mathfrak{h}}=\bigsqcup X_{W^{\prime \prime}, \mathfrak{h}}^{\circ},
$$

where the union is over a set of representatives of the $W$-conjugacy classes of the parabolic subgroups $W^{\prime \prime}$ of $W$ which contain $W^{\prime}$. Further, the quotient $X_{W^{\prime}, \mathfrak{h}} / W$ is an irreducible closed subset of $\mathfrak{h} / W$.

2.3. Induction and restriction functors on $\mathcal{O}(W)$. Fix an element $b \in \mathfrak{h}$. Let $W_{b} \subset W$ be the stabilizer of $b$, and

$$
\pi_{b}: \mathfrak{h} \rightarrow \mathfrak{h} / \mathfrak{h}^{W_{b}}
$$

be the obvious projection onto the reflection representation of $W_{b}$. The parabolic induction and restriction functors associated with the point $b$ are respectively the functors 2]

$$
\operatorname{Ind}_{b}: \mathcal{O}\left(W_{b}, \mathfrak{h} / \mathfrak{h}^{W_{b}}\right) \rightarrow \mathcal{O}(W, \mathfrak{h}), \quad \operatorname{Res}_{b}: \mathcal{O}(W, \mathfrak{h}) \rightarrow \mathcal{O}\left(W_{b}, \mathfrak{h} / \mathfrak{h}^{W_{b}}\right) .
$$

Since the functors $\operatorname{Ind}_{b}$, $\operatorname{Res}_{b}$ do not depend on $b$ up to isomorphism, see [2, sec. 3.7], we may write

$$
{ }^{\mathcal{O}} \operatorname{Ind}_{W_{b}}^{W}=\operatorname{Ind}_{b}, \quad{ }^{\mathcal{O}} \operatorname{Res}_{W_{b}}^{W}=\operatorname{Res}_{b}
$$

if this does not create any confusion. The support of a module $M$ in $\mathcal{O}(W, \mathfrak{h})$ is the support of $M$ regarded as a $\mathbb{C}[\mathfrak{h}]$-module. It is a closed subset $\operatorname{Supp}(M) \subset \mathfrak{h}$. By [13, thm. 6.8] for any simple module $L$ in $\mathcal{O}(W, \mathfrak{h})$ we have $\operatorname{Supp}(L)=X_{W^{\prime}, \mathfrak{h}}$ for some parabolic subgroup $W^{\prime} \subset W$. For $b \in X_{W^{\prime}, \mathfrak{h}}^{\circ}$ the $\operatorname{module}^{\operatorname{Res}_{b}}(L)$ is a nonzero finite-dimensional module. See [2, sec. 3.8]. The support of a module is the union of the supports of all its constituents. So the support of any module in $\mathcal{O}(W, \mathfrak{h})$ is a union of $X_{W^{\prime}, \mathfrak{h}}$ 's. Let us consider the behavior of the support under restriction.

Proposition 2.2. Let $W^{\prime} \subset W$ be a parabolic subgroup. Let $\mathfrak{h}^{\prime}$ be the reflection representation of $W^{\prime}$. Let $X \subset \mathfrak{h}$ be the support of a module $M$ in $\mathcal{O}(W, \mathfrak{h})$. Let $X^{\prime} \subset \mathfrak{h}^{\prime}$ be the support of the module $M^{\prime}={ }^{\mathcal{O}} \operatorname{Res}_{W^{\prime}}^{W}(M)$.

(a) We have $M^{\prime} \neq 0$ if and only if $X_{W^{\prime}, \mathfrak{h}} \subset X$.

(b) Assume that $X=X_{W^{\prime \prime}, \mathfrak{h}}$ with $W^{\prime \prime} \subset W$ a parabolic subgroup. If $M^{\prime} \neq 0$, then $W^{\prime \prime}$ is $W$-conjugate to a subgroup of $W^{\prime}$ and we have

$$
X^{\prime}=\bigcup_{W_{1}} X_{W_{1}, \mathfrak{h}^{\prime}}=\bigsqcup_{W_{1}} X_{W_{1}, \mathfrak{h}^{\prime}}^{\circ},
$$


where $W_{1}$ runs over a set of representatives of the $W^{\prime}$-conjugacy classes of parabolic subgroups of $W^{\prime}$ containing a subgroup $W$-conjugated to $W^{\prime \prime}$.

Proof. Part $(a)$ is immediate from the definition of the restriction, because for $b \in \mathfrak{h}$ it implies that $\operatorname{Res}_{b}(M) \neq 0$ if and only if $b \in X$; see also Remark 2.3 below. Now we prove $(b)$. For a parabolic subgroup $W_{1} \subset W^{\prime}$ we have

$$
\begin{aligned}
X_{W_{1}, \mathfrak{h}^{\prime}} \subset X^{\prime} & \Longleftrightarrow{ }^{\mathcal{O}} \operatorname{Res}_{W_{1}}^{W^{\prime}}\left(M^{\prime}\right) \neq 0 \\
& \Longleftrightarrow{ }^{\mathcal{O}} \operatorname{Res}_{W_{1}}^{W}(M) \neq 0 \\
& \Longleftrightarrow X_{W_{1}, \mathfrak{h}} \subset X_{W^{\prime \prime}, \mathfrak{h}} .
\end{aligned}
$$

Here the first and third equivalence follow from $(a)$, while the second one follows from the transitivity of the restriction functor [33, cor. 2.5]. Therefore $X_{W_{1}, \mathfrak{h}^{\prime}}^{\circ} \subset X^{\prime}$ if and only if $X_{W_{1}, \mathfrak{h}^{\prime}} \subset X^{\prime}$ if and only if $W_{1}$ contains a subgroup $W$-conjugate to $W^{\prime \prime}$.

Remark 2.3. For any closed point $b$ of a scheme $X$ we denote by $X_{b}^{\wedge}$ the completion of $X$ at $b$ (a formal scheme). Assume that $M^{\prime}={ }^{\mathcal{O}} \operatorname{Res}_{W^{\prime}}^{W}(M)$ is nonzero. We define $X, X^{\prime}$ as in the proposition above. Let $\pi$ be the canonical projection $\mathfrak{h} \rightarrow \mathfrak{h}^{\prime}=$ $\mathfrak{h} / \mathfrak{h}^{W^{\prime}}$. For $b \in X_{W^{\prime}, \mathfrak{h}}^{\circ}$ the definition of the restriction functor yields the following formula:

$$
0 \in \pi^{-1}\left(X^{\prime}\right), \quad X_{b}^{\wedge}=b+\pi^{-1}\left(X^{\prime}\right)_{0}^{\wedge}
$$

Next, we consider the behavior of the support under induction. Before this we need the following two lemmas. The $\mathbb{C}$-vector space $[\mathcal{O}(W)]$ is spanned by the set $\left\{\left[\Delta_{\chi}\right]: \chi \in \operatorname{Irr}(\mathbb{C} W)\right\}$. Thus there is a unique $\mathbb{C}$-linear isomorphism

$$
\text { spe }:[\operatorname{Rep}(\mathbb{C} W)] \rightarrow[\mathcal{O}(W)], \quad[\chi] \mapsto\left[\Delta_{\chi}\right] .
$$

The parabolic induction/restriction functor is exact. We'll need the following lemma 2 .

Lemma 2.4. Let $W^{\prime} \subset W$ be a parabolic subgroup. Let $\mathfrak{h}^{\prime}$ be the reflection representation of $W^{\prime}$. Under the isomorphism (2.1) the maps

$$
{ }^{\mathcal{O}} \operatorname{Ind}_{W^{\prime}}^{W}:\left[\mathcal{O}\left(W^{\prime}, \mathfrak{h}^{\prime}\right)\right] \rightarrow[\mathcal{O}(W, \mathfrak{h})], \quad{ }^{\mathcal{O}} \operatorname{Res}_{W^{\prime}}^{W}:[\mathcal{O}(W, \mathfrak{h})] \rightarrow\left[\mathcal{O}\left(W^{\prime}, \mathfrak{h}^{\prime}\right)\right]
$$

coincide with the induction and restriction

$$
\operatorname{Ind}_{W^{\prime}}^{W}:\left[\operatorname{Rep}\left(\mathbb{C} W^{\prime}\right)\right] \rightarrow[\operatorname{Rep}(\mathbb{C} W)], \quad \operatorname{Res}_{W^{\prime}}^{W}:[\operatorname{Rep}(\mathbb{C} W)] \rightarrow\left[\operatorname{Rep}\left(\mathbb{C} W^{\prime}\right)\right] .
$$

We'll also need the following version of the Mackey induction/restriction theorem. First, observe that for any parabolic subgroup $W^{\prime} \subset W$ and any $x \in W$ there is a canonical $\mathbb{C}$-algebra isomorphism

$$
\varphi_{x}: H\left(W^{\prime}\right) \rightarrow H\left(x^{-1} W^{\prime} x\right), w \mapsto x^{-1} w x, f \mapsto x^{-1} f x, f^{\prime} \mapsto x^{-1} f^{\prime} x,
$$

for $w \in W^{\prime}, f \in R_{x}, f^{\prime} \in R_{y}$. It yields an exact functor

$$
\mathcal{O}\left(W^{\prime}\right) \rightarrow \mathcal{O}\left(x^{-1} W^{\prime} x\right), \quad M \mapsto{ }^{x} M,
$$

where ${ }^{x} M$ is the $H\left(x^{-1} W^{\prime} x\right)$-module obtained by twisting the $H\left(W^{\prime}\right)$-action on $M$ by $\varphi_{x}$. 
Lemma 2.5. Let $W^{\prime}, W^{\prime \prime} \subset W$ be parabolic subgroups. Let $\mathfrak{h}^{\prime}, \mathfrak{h}^{\prime \prime}$ be the reflection representations of $W^{\prime}, W^{\prime \prime}$. For $M \in \mathcal{O}\left(W^{\prime}, \mathfrak{h}^{\prime}\right)$ we have the following formula in $\left[\mathcal{O}\left(W^{\prime \prime}, \mathfrak{h}^{\prime \prime}\right)\right]$ :

$$
{ }^{\mathcal{O}} \operatorname{Res}_{W^{\prime \prime}}^{W} \circ{ }^{\mathcal{O}} \operatorname{Ind}_{W^{\prime}}^{W}([M])=\sum_{x}{ }^{\mathcal{O}} \operatorname{Ind}_{W^{\prime \prime} \cap x^{-1} W^{\prime} x}^{W^{\prime \prime}}{ }^{x}\left({ }^{\mathcal{O}} \operatorname{Res}_{x W^{\prime \prime} x^{-1} \cap W^{\prime}}^{W^{\prime}}([M])\right),
$$

where $x$ runs over a set of representatives of the cosets in $W^{\prime} \backslash W / W^{\prime \prime}$.

Proof. Use Lemma 2.4 and the usual Mackey induction/restriction theorem associated with the triplet of groups $W, W^{\prime}, W^{\prime \prime}$.

Remark 2.6. For a future use, note that the left-hand side of (2.2) is zero if and only if each term in the sum of the right-hand side is zero, because each of these terms is the class of a module in $\mathcal{O}\left(W^{\prime \prime}, \mathfrak{h}^{\prime \prime}\right)$.

Now, we can prove the following proposition.

Proposition 2.7. Let $W^{\prime \prime} \subset W^{\prime} \subset W$ be parabolic subgroups. Let $\mathfrak{h}^{\prime}$ be the reflection representation of $W^{\prime}$. For a simple module $L \in \mathcal{O}\left(W^{\prime}, \mathfrak{h}^{\prime}\right)$ with $\operatorname{Supp}(L)=$ $X_{W^{\prime \prime}, \mathfrak{h}^{\prime}}$, we have

$$
{ }^{\mathcal{O}} \operatorname{Ind}_{W^{\prime}}^{W}(L) \neq 0, \quad \operatorname{Supp}\left({ }^{\mathcal{O}} \operatorname{Ind}_{W^{\prime}}^{W}(L)\right)=X_{W^{\prime \prime}, \mathfrak{h}} .
$$

Proof. First, note that ${ }^{\mathcal{O}} \operatorname{Ind}_{W^{\prime}}^{W}(L) \neq 0$ by Lemma 2.5, because

$$
{ }^{\mathcal{O}} \operatorname{Res}_{W^{\prime}}^{W}{ }^{\mathcal{O}} \operatorname{Ind}_{W^{\prime}}^{W}([L])=[L]+[M]
$$

for some $M \in \mathcal{O}\left(W^{\prime}, \mathfrak{h}^{\prime}\right)$ and $[L] \neq 0$. We abbreviate $M={ }^{\mathcal{O}} \operatorname{Ind}_{W^{\prime}}^{W}(L)$. To compute the support of $M$ we first check that

$$
X_{W^{\prime \prime}, \mathfrak{h}} \subset \operatorname{Supp}(M) .
$$

By Proposition 2.2 we have

$$
\begin{aligned}
X_{W^{\prime \prime}, \mathfrak{h}} \subset \operatorname{Supp}(M) & \Longleftrightarrow X_{W^{\prime \prime}, \mathfrak{h}}^{\circ} \subset \operatorname{Supp}(M) \\
& \Longleftrightarrow{ }^{\mathcal{O}} \operatorname{Res}_{W^{\prime \prime}}^{W}(M) \neq 0 .
\end{aligned}
$$

By Remark 2.6 the last equality holds if and only if

$$
{ }^{\mathcal{O}} \operatorname{Res}_{x W^{\prime \prime} x^{-1} \cap W^{\prime}}^{W^{\prime}}(L) \neq 0
$$

for some $x \in W$. This identity is indeed true for $x=1$ because $W^{\prime \prime} \subset W^{\prime}$ and

$$
X_{W^{\prime \prime}, \mathfrak{h}^{\prime}}=\operatorname{Supp}(L) \Rightarrow{ }^{\mathcal{O}} \operatorname{Res}_{W^{\prime \prime}}^{W^{\prime}}(L) \neq 0 .
$$

Next we prove the inclusion

$$
\operatorname{Supp}(M) \subset X_{W^{\prime \prime}, \mathfrak{h}}
$$

Any point $b$ of $\mathfrak{h} \backslash X_{W^{\prime \prime}, \mathfrak{h}}$ is contained in the set $X_{W^{\prime \prime \prime}, \mathfrak{h}}^{\circ}$ for some parabolic subgroup $W^{\prime \prime \prime} \subset W$ such that $W^{\prime \prime}$ is not conjugate to a subgroup of $W^{\prime \prime \prime}$ : it suffices to set $W^{\prime \prime \prime}=W_{b}$. We must check that for such a subgroup $W^{\prime \prime \prime} \subset W$ we have

$$
X_{W^{\prime \prime \prime}, \mathfrak{h}}^{\circ} \not \subset \operatorname{Supp}(M) \text {. }
$$

By Proposition 2.2 it is enough to check that

$$
{ }^{\mathcal{O}} \operatorname{Res}_{W^{\prime \prime \prime}}^{W}(M)=0 .
$$


Now, by Lemma 2.5 we have the following formula in $\left[\mathcal{O}\left(W^{\prime \prime \prime}, \mathfrak{h}\right)\right]$ :

$$
{ }^{\mathcal{O}} \operatorname{Res}_{W^{\prime \prime \prime}}^{W}([M])=\sum_{x}{ }^{\mathcal{O}} \operatorname{Ind}_{W^{\prime \prime \prime} \cap x^{-1} W^{\prime} x}^{W^{\prime \prime \prime}} \circ{ }^{x}\left({ }^{\mathcal{O}} \operatorname{Res}_{x W^{\prime \prime \prime} x^{-1} \cap W^{\prime}}^{W^{\prime}}([L])\right) .
$$

Here $x$ runs over a set of representatives of the cosets in $W^{\prime} \backslash W / W^{\prime \prime \prime}$. Since $W^{\prime \prime}$ is not conjugate to a subgroup of $W^{\prime \prime \prime}$ it is a fortiori not conjugate to a subgroup of $x W^{\prime \prime \prime} x^{-1} \cap W^{\prime}$; i.e., we have

$$
X_{x W^{\prime \prime \prime} x^{-1} \cap W^{\prime}, \mathfrak{h}^{\prime}}^{\circ} \cap X_{W^{\prime \prime}, \mathfrak{h}^{\prime}}=\emptyset .
$$

Therefore Proposition 2.2 yields

$$
{ }^{\mathcal{O}} \operatorname{Res}_{x W^{\prime \prime \prime} x^{-1} \cap W^{\prime}}^{W^{\prime}}(L)=0,
$$

because $\operatorname{Supp}(L)=X_{W^{\prime \prime}, \mathfrak{h}^{\prime}}$. This implies that

$$
{ }^{\mathcal{O}} \operatorname{Res}_{W^{\prime \prime \prime}}^{W}([M])=0 .
$$

Hence we have also

$$
{ }^{\mathcal{O}} \operatorname{Res}_{W^{\prime \prime \prime}}^{W}(M)=0
$$

We are done.

\section{The Cyclotomic Rational DAHA}

3.1. Combinatorics. For a sequence $\lambda=\left(\lambda_{1}, \lambda_{2}, \ldots\right)$ of integers $\geqslant 0$ we set $|\lambda|=$ $\lambda_{1}+\lambda_{2}+\cdots$. Let

$$
\Lambda(\ell, n)=\left\{\lambda=\left(\lambda_{1}, \lambda_{2}, \ldots \lambda_{\ell}\right) \in \mathbb{N}^{\ell}:|\lambda|=n\right\} .
$$

It is the set of compositions of $n$ with $\ell$ parts. Let $\mathcal{P}_{n}$ be the set of partitions of $n$, i.e., the set of nonincreasing sequences $\lambda$ of integers $>0$ with sum $|\lambda|=n$. We write $\lambda^{\prime}$ for the transposed partition and $l(\lambda)$ for its length, i.e., for the number of parts in $\lambda$. We write also

$$
z_{\lambda}=\prod_{i \geqslant 1} i^{m_{i}} m_{i} !
$$

where $m_{i}$ is the number of parts of $\lambda$ equal to $i$. Given a positive integer $m$ and a partition $\lambda$ we write also

$$
m \lambda=\left(m \lambda_{1}, m \lambda_{2}, \ldots\right) .
$$

To any partition we associate a Young diagram, which is a collection of rows of square boxes with $\lambda_{i}$ boxes in the $i$-th row, $i=1, \ldots, l(\lambda)$. A box in a Young diagram is called a node. The coordinate of the $j$-th box in the $i$-th row is the pair of integers $(i, j)$. The content of the node of coordinate $(i, j)$ is the integer $j-i$. Let the set $\mathcal{P}_{0}$ consist of a single element, the unique partition of zero, which we denote by 0 . Let $\mathcal{P}=\bigsqcup_{n \geqslant 0} \mathcal{P}_{n}$ be the set of all partitions. We'll abbreviate $\mathbb{Z}_{\ell}=\mathbb{Z} / \ell \mathbb{Z}$. Let $\mathcal{P}^{\ell}$ be the set of $\ell$-partitions, i.e., the set of all partition-valued functions on $\mathbb{Z}_{\ell}$. Let $\mathcal{P}_{n}^{\ell}$ be the subset of $\ell$-tuples $\lambda=(\lambda(p))$ of partitions with $|\lambda|=\sum_{p}|\lambda(p)|=n$. Let $\Gamma$ be the group of the $\ell$-th roots of 1 in $\mathbb{C}^{\times}$. We define the sets $\mathcal{P}^{\Gamma}, \mathcal{P}_{n}^{\Gamma}$ of partition-valued functions on $\Gamma$ in the same way. 
3.2. The complex reflection group $\Gamma_{n}$. Fix nonnegative integers $\ell, n$. Unless specified otherwise we'll always assume that $\ell, n \neq 0$. Let $\mathfrak{S}_{n}$ be the symmetric group on $n$ letters and $\Gamma_{n}$ be the semi-direct product $\mathfrak{S}_{n} \ltimes \Gamma^{n}$, where $\Gamma^{n}$ is the Cartesian product of $n$ copies of $\Gamma$. We write also $\mathfrak{S}_{0}=\Gamma^{0}=\Gamma_{0}=\{1\}$. For $\gamma \in \Gamma$ let $\gamma_{i} \in \Gamma^{n}$ be the element with $\gamma$ at the $i$-th place and with 1 at the other places. Let $s_{i j}$ be the transposition $(i, j)$ in $\mathfrak{S}_{n}$. We'll abbreviate $s_{i}=s_{i, i+1}$. Write $s_{i j}^{\gamma}=s_{i j} \gamma_{i} \gamma_{j}^{-1}$ for $\gamma \in \Gamma, i \neq j$. For $p \in \mathbb{Z}_{\ell}$ let $\chi_{p}: \Gamma \rightarrow \mathbb{C}^{\times}$be the character $\gamma \mapsto \gamma^{p}$. The assignment $p \mapsto \chi_{p}$ identifies $\mathbb{Z}_{\ell}$ with the group of characters of $\Gamma$. The group $\Gamma_{n}$ is a complex reflection group. For $\ell>1$ it acts on the vector space $\mathfrak{h}=\mathbb{C}^{n}$ via the reflection representation. For $\ell=1$ the reflection representation is given by the permutation of coordinates on the hyperplane

$$
\mathbb{C}_{0}^{n}=\left\{x_{1}+\cdots+x_{n}=0\right\} \subset \mathbb{C}^{n} .
$$

We'll be interested in the following subgroups of $\Gamma_{n}$.

- To a composition $\nu$ of $n$ we associate the set

$$
I=\{1,2, \ldots, n-1\} \backslash\left\{\nu_{1}, \nu_{1}+\nu_{2}, \ldots\right\} .
$$

Let $\Gamma_{\nu}=\mathfrak{S}_{\nu} \ltimes \Gamma^{n}$, where $\mathfrak{S}_{\nu}=\mathfrak{S}_{I}$ is the subgroup of $\mathfrak{S}_{n}$ generated by the simple reflections $s_{i, i+1}$ with $i \in I$.

- For integers $m, n \geqslant 0$ and a composition $\nu$ we set $\Gamma_{n, \nu}=\Gamma_{n} \times \mathfrak{S}_{\nu}$. If $\nu=(m)$ we abbreviate $\Gamma_{n, m}=\Gamma_{n,(m)}=\Gamma_{n} \times \mathfrak{S}_{m}$. Any parabolic subgroup of $\Gamma_{n}$ is conjugate to $\Gamma_{l, \nu}$ for some $l, \nu$ with $l+|\nu| \leqslant n$.

3.3. Definition of the cyclotomic rational DAHA. Let $\mathfrak{h}=\mathbb{C}^{n}$ be the reflection representation of $\Gamma_{n}$. Denote by $y_{1}, \ldots, y_{n}$ the standard basis of $\mathfrak{h}$, and by $x_{1}, \ldots, x_{n}$ its dual basis in $\mathfrak{h}^{*}$. The actions of the group $\Gamma_{n}$ on $\mathfrak{h}$ and on $\mathfrak{h}^{*}$ are given as follows: for distinct $i, j, k$ we have

$$
\begin{gathered}
\gamma_{i}\left(y_{i}\right)=\gamma y_{i}, \quad \gamma_{i}\left(y_{j}\right)=y_{j}, \quad s_{i j}\left(y_{i}\right)=y_{j}, \quad s_{i j}\left(y_{k}\right)=y_{k}, \\
\gamma_{i}\left(x_{i}\right)=\gamma^{-1} x_{i}, \quad \gamma_{i}\left(x_{j}\right)=x_{j}, \quad s_{i j}\left(x_{i}\right)=x_{j}, \quad s_{i j}\left(x_{k}\right)=x_{k} .
\end{gathered}
$$

Fix $k \in \mathbb{C}$ and $c_{\gamma} \in \mathbb{C}$ for each $\gamma \in \Gamma$. We can define the algebra $H(W)=H(W, \mathfrak{h})$ for $W=\Gamma_{n}$. We'll call $H\left(\Gamma_{n}\right)$ the cyclotomic rational DAHA. It is the quotient of the smash product of $\mathbb{C} \Gamma_{n}$ and the tensor algebra of $\mathfrak{h} \oplus \mathfrak{h}^{*}$ by the relations

$$
\begin{gathered}
{\left[y_{i}, x_{i}\right]=-k \sum_{j \neq i} \sum_{\gamma \in \Gamma} s_{i j}^{\gamma}-\sum_{\gamma \in \Gamma} c_{\gamma} \gamma_{i}, \quad c_{1}=-1,} \\
{\left[y_{i}, x_{j}\right]=k \sum_{\gamma \in \Gamma} \gamma s_{i j}^{\gamma} \quad \text { if } i \neq j,} \\
{\left[x_{i}, x_{j}\right]=\left[y_{i}, y_{j}\right]=0 .}
\end{gathered}
$$

Let $R_{x}, R_{y}$ be the subalgebras generated by $x_{1}, x_{2}, \ldots, x_{n}$ and $y_{1}, y_{2}, \ldots, y_{n}$ respectively. We'll identify $\mathfrak{h}, \mathfrak{h}^{*}$ with the maximal spectrum of $R_{x}, R_{y}$. We'll use another presentation where the parameters are $h, h_{p}$ with $p \in \mathbb{Z}_{\ell}$ where $k=-h$ and $-c_{\gamma}=\sum_{p \in \mathbb{Z}_{\ell}} \gamma^{-p} h_{p}$. Note that $1=\sum_{p} h_{p}$. 
3.4. The Lie algebras $\widehat{\mathfrak{s l}}_{\ell}$ and $\widetilde{\mathfrak{s l}}_{\ell}$. Given complex numbers $h_{p}, p \in \mathbb{Z}_{\ell}$, with $\sum_{p} h_{p}=1$, it is convenient to consider the following level 1 weight

$$
\Lambda=\sum_{p} h_{p} \omega_{p}
$$

Here the $\omega_{p}$ 's are the fundamental weights of the affine Lie algebra

$$
\widehat{\mathfrak{s l}}_{\ell}=\left(\mathfrak{s l}_{\ell} \otimes \mathbb{C}\left[\varpi, \varpi^{-1}\right]\right) \oplus \mathbb{C} \mathbf{1},
$$

where $\mathbf{1}$ is a central element and the Lie bracket is given by

$$
\left[x \otimes \varpi^{r}, y \otimes \varpi^{s}\right]=[x, y] \otimes \varpi^{r+s}+r(x, y) \delta_{r,-s} \mathbf{1}, \quad(x, y)=\tau\left(x y^{t}\right),
$$

where $y \mapsto y^{t}$ is the transposition and $\tau$ is the trace. The affine Lie algebra $\widehat{\mathfrak{s l}}_{\ell}$ is generated by the symbols $e_{p}, f_{p}, p=0, \ldots, \ell-1$, satisfying the Serre relations. For $p \neq 0$ we have

$$
e_{p}=e_{p, p+1} \otimes 1, \quad e_{0}=e_{\ell, 1} \otimes \varpi, \quad f_{p}=e_{p+1, p} \otimes 1, \quad f_{0}=e_{1, \ell} \otimes \varpi^{-1},
$$

where $e_{p, q}$ is the usual elementary matrix in $\mathfrak{s l}_{\ell}$. We'll also use the extended affine Lie algebras $\widetilde{\mathfrak{s l}}_{\ell}$, obtained by adding to $\widehat{\mathfrak{s l}}_{\ell}$ the 1-dimensional vector space spanned by the scaling element $D$ such that $\left[D, x \otimes \varpi^{r}\right]=r x \otimes \varpi^{r}$ and $[D, \mathbf{1}]=0$. Let $\delta$ denote the dual of $D$, i.e., the smallest positive imaginary root. We equip the space of linear forms on the Cartan subalgebra of $\widetilde{\mathfrak{s l}}_{\ell}$ with the pairing such that

$$
\left\langle\omega_{p}, \omega_{q}\right\rangle=\min (p, q)-p q / \ell, \quad\left\langle\omega_{p}, \delta\right\rangle=1, \quad\langle\delta, \delta\rangle=0 .
$$

Let $U\left(\widehat{\mathfrak{s l}}_{\ell}\right)$ be the enveloping algebra of $\widehat{\mathfrak{s l}}_{\ell}$, and let $U^{-}\left(\widehat{\mathfrak{s l}}_{\ell}\right)$ be the subalgebra generated by the elements $f_{p}$ with $p=0, \ldots, \ell-1$. For $r \geqslant 0$ we write $U^{-}\left(\widehat{\mathfrak{s l}}_{\ell}\right)_{r}$ for the subspace of $U^{-}\left(\widehat{\mathfrak{s}}_{\ell}\right)$ spanned by the monomials whose weight is the sum of $r$ negative simple roots.

3.5. Representations of $\mathfrak{S}_{n}, \Gamma_{n}$. The set of isomorphism classes of irreducible $\mathfrak{S}_{n}$-modules is

$$
\operatorname{Irr}\left(\mathbb{C S}_{n}\right)=\left\{\bar{L}_{\lambda} ; \lambda \in \mathcal{P}_{n}\right\}
$$

see [27, sec. I.9]. The set of isomorphism classes of irreducible $\Gamma_{n}$-modules is

$$
\operatorname{Irr}\left(\mathbb{C} \Gamma_{n}\right)=\left\{\bar{L}_{\lambda} ; \lambda \in \mathcal{P}_{n}^{\ell}\right\}
$$

where $\bar{L}_{\lambda}$ is defined as follows. Write $\lambda=(\lambda(p))$. The tuple of positive integers $\nu_{\lambda}=(|\lambda(p)|)$ is a composition in $\Lambda(\ell, n)$. Let

$$
\bar{L}_{\lambda(p)}\left(\chi_{p-1}\right)^{\otimes|\lambda(p)|} \in \operatorname{Irr}\left(\mathbb{C} \Gamma_{|\lambda(p)|}\right)
$$

be the tensor product of the $\mathfrak{S}_{|\lambda(p)|}$-module $\bar{L}_{\lambda(p)}$ and the one-dimensional $\Gamma^{|\lambda(p)|_{-}}$ module $\left(\chi_{p-1}\right)^{\otimes|\lambda(p)|}$. The $\Gamma_{n}$-module $\bar{L}_{\lambda}$ is given by

$$
\bar{L}_{\lambda}=\operatorname{Ind}_{\Gamma_{\nu_{\lambda}}}^{\Gamma_{n}}\left(\bar{L}_{\lambda(1)} \chi_{\ell}^{\otimes|\lambda(1)|} \otimes \bar{L}_{\lambda(2)} \chi_{1}^{\otimes|\lambda(2)|} \otimes \cdots \otimes \bar{L}_{\lambda(\ell)} \chi_{\ell-1}^{\otimes|\lambda(\ell)|}\right) .
$$


3.6. The category $\mathcal{O}\left(\Gamma_{n}\right)$. Consider the $\mathbb{C}$-algebra $H\left(\Gamma_{n}\right)$ with the parameter $\Lambda$ in (3.2). The category $\mathcal{O}$ of $H\left(\Gamma_{n}\right)$ is the quasi-hereditary category $\mathcal{O}\left(\Gamma_{n}\right)$. The standard modules are the induced modules

$$
\Delta_{\lambda}=\operatorname{Ind}_{\Gamma_{n} \ltimes R_{y}}^{H\left(\Gamma_{n}\right)}\left(\bar{L}_{\lambda}\right), \quad \lambda \in \mathcal{P}_{n}^{\ell} .
$$

Here $\bar{L}_{\lambda}$ is viewed as a $\Gamma_{n} \ltimes R_{y}$-module such that $y_{1}, \ldots, y_{n}$ act trivially. Let $L_{\lambda}$, $P_{\lambda}$ denote the top and the projective cover of $\Delta_{\lambda}$. Recall the $\mathbb{C}$-linear isomorphism

$$
\text { spe : }\left[\operatorname{Rep}\left(\mathbb{C} \Gamma_{n}\right)\right] \rightarrow\left[\mathcal{O}\left(\Gamma_{n}\right)\right], \quad\left[\bar{L}_{\lambda}\right] \mapsto\left[\Delta_{\lambda}\right] .
$$

To avoid cumbersome notation for induction/restriction functors in

$$
\mathcal{O}(\Gamma)=\bigoplus_{n \geqslant 0} \mathcal{O}\left(\Gamma_{n}\right)
$$

we'll abbreviate

$$
\begin{aligned}
& { }^{\mathcal{O}} \operatorname{Ind}_{n}={ }^{\mathcal{O}} \operatorname{Ind}_{\Gamma_{n-1}}^{\Gamma_{n}}, \quad{ }^{\mathcal{O}} \operatorname{Res}_{n}={ }^{\mathcal{O}} \operatorname{Res}_{\Gamma_{n-1}}^{\Gamma_{n}}, \\
& { }^{\mathcal{O}} \operatorname{Ind}_{n,\left(m^{r}\right)}={ }^{\mathcal{O}} \operatorname{Ind}_{\Gamma_{n,\left(m^{r}\right)} \Gamma_{n+m r}}, \quad{ }^{\mathcal{O}} \operatorname{Res}_{n,\left(m^{r}\right)}={ }^{\mathcal{O}} \operatorname{Res}_{\Gamma_{n,\left(m^{r}\right)}}^{\Gamma_{n+m r}}, \\
& { }^{\mathcal{O}} \operatorname{Ind}_{n, m r}={ }^{\mathcal{O}} \operatorname{Ind}_{\Gamma_{n, m r}}^{\Gamma_{n+m r}}, \quad{ }^{\mathcal{O}} \operatorname{Res}_{n, m r}={ }^{\mathcal{O}} \operatorname{Res}_{\Gamma_{n, m r}}^{\Gamma_{n+m r}} .
\end{aligned}
$$

We write also

$$
\begin{aligned}
{ }^{\mathcal{O}} \operatorname{Ind}_{\left(m^{r}\right)} & ={ }^{\mathcal{O}} \operatorname{Ind}_{\mathfrak{S}_{m}^{r r}}^{\mathfrak{S}_{m r}^{r}}: \mathcal{O}\left(\mathfrak{S}_{m}^{r}\right) \rightarrow \mathcal{O}\left(\mathfrak{S}_{m r}\right), \\
{ }^{\mathcal{O}} \operatorname{Res} & ={ }^{\mathcal{O}} \operatorname{Res}_{\mathfrak{S}_{m}^{r}}^{\mathfrak{S}_{m r}^{r}}: \mathcal{O}\left(\mathfrak{S}_{m r}\right) \rightarrow \mathcal{O}\left(\mathfrak{S}_{m}^{r}\right) .
\end{aligned}
$$

3.7. The functor $\mathbf{K Z}$. For $\zeta \in \mathbb{C}^{\times}$and $v_{1}, v_{2}, \ldots, v_{\ell} \in \mathbb{C}^{\times}$let $\mathbf{H}_{\zeta}(n, \ell)$ be the cyclotomic Hecke algebra associated with $\Gamma_{n}$ and the parameters $\zeta, v_{1}, \ldots, v_{\ell}$; see Section A.2. We'll abbreviate $\mathbf{H}\left(\Gamma_{n}\right)=\mathbf{H}_{\zeta}(n, \ell)$. Assume that

$$
\zeta=\exp (2 i \pi h), \quad v_{p}=v_{1} \exp \left(-2 i \pi\left(h_{1}+h_{2}+\cdots+h_{p-1}\right)\right) .
$$

Then the KZ-functor [14 is a quotient functor

$$
\mathrm{KZ}: \mathcal{O}\left(\Gamma_{n}\right) \rightarrow \operatorname{Rep}\left(\mathbf{H}\left(\Gamma_{n}\right)\right) .
$$

Since KZ is a quotient functor, it admits a right adjoint functor

$$
S: \operatorname{Rep}\left(\mathbf{H}\left(\Gamma_{n}\right)\right) \rightarrow \mathcal{O}\left(\Gamma_{n}\right)
$$

such that $\mathrm{KZ} \circ S=\mathbf{1}$. By [14, thm. 5.3], for each projective module $Q \in \mathcal{O}\left(\Gamma_{n}\right)$ the canonical adjunction morphism $\mathbf{1} \rightarrow S \circ \mathrm{KZ}$ yields an isomorphism

$$
Q \rightarrow S(\mathrm{KZ}(Q)) \text {. }
$$

3.8. The functor $R$. Let $\mathbf{H}_{\zeta}(m)$ be the Hecke algebra of $G L_{m}$ over $\mathbb{C}$; see Section A.2. Let $\mathbf{S}_{\zeta}(m)$ be the $\zeta$-Schur algebra over $\mathbb{C}$; see Appendix $\mathbb{B}$ The module categories of $\mathbf{S}_{\zeta}(m), \mathbf{H}_{\zeta}(m)$ are related through the Schur functor

$$
\Phi^{*}: \operatorname{Rep}\left(\mathbf{S}_{\zeta}(m)\right) \rightarrow \operatorname{Rep}\left(\mathbf{H}_{\zeta}(m)\right) .
$$

Set

$\Lambda(m)_{+}=\Lambda(m, m) \cap \mathbb{Z}_{+}^{m}, \mathbb{Z}_{+}^{m}=\left\{\lambda=\left(\lambda_{1}, \lambda_{2}, \ldots, \lambda_{m}\right) \in \mathbb{Z}^{m}: \lambda_{1} \geqslant \lambda_{2} \geqslant \cdots \geqslant \lambda_{m}\right\}$.

The category $\operatorname{Rep}\left(\mathbf{S}_{\zeta}(m)\right)$ is quasi-hereditary with respect to the dominance order, the standard objects being the modules $\Delta_{\lambda}^{S}$ with $\lambda \in \Lambda(m)_{+}$. The comultiplication $\Delta$ yields a bifunctor (B.6)

$$
\dot{\otimes}: \operatorname{Rep}\left(\mathbf{S}_{\zeta}(m)\right) \otimes \operatorname{Rep}\left(\mathbf{S}_{\zeta}\left(m^{\prime}\right)\right) \rightarrow \operatorname{Rep}\left(\mathbf{S}_{\zeta}\left(m+m^{\prime}\right)\right) .
$$


Now, assume that $h$ is a negative rational number with denominator $d$ and let $\zeta \in \mathbb{C}^{\times}$be a primitive $d$-th root of 1 . Recall that $h$ is the parameter of the $\mathbb{C}$ algebra $H\left(\mathfrak{S}_{m}\right)$. If $h \notin 1 / 2+\mathbb{Z}$, then Rouquier's functor [32] is an equivalence of quasi-hereditary categories

$$
R: \mathcal{O}\left(\mathfrak{S}_{m}\right) \rightarrow \operatorname{Rep}\left(\mathbf{S}_{\zeta}(m)\right), \quad \Delta_{\lambda} \mapsto \Delta_{\lambda}^{S}
$$

such that $\mathrm{KZ}=\Phi^{*} \circ R$. For $m=m^{\prime}+m^{\prime \prime}$ we have a canonical equivalence of categories $\mathcal{O}\left(\mathfrak{S}_{m^{\prime}}\right) \otimes \mathcal{O}\left(\mathfrak{S}_{m^{\prime \prime}}\right)=\mathcal{O}\left(\mathfrak{S}_{m^{\prime}} \times \mathfrak{S}_{m^{\prime \prime}}\right)$ and the induction yields a bifunctor

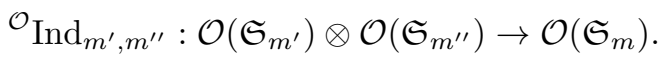

We'll abbreviate

$$
\mathcal{O}(\mathfrak{S})=\bigoplus_{n \geqslant 0} \mathcal{O}\left(\mathfrak{S}_{n}\right), \quad \operatorname{Rep}\left(\mathbf{S}_{\zeta}\right)=\bigoplus_{n \geqslant 0} \operatorname{Rep}\left(\mathbf{S}_{\zeta}(n)\right)
$$

Proposition 3.1. For $h \notin 1 / 2+\mathbb{Z}$ the functor $R$ is a tensor equivalence $\mathcal{O}(\mathfrak{S}) \rightarrow$ $\operatorname{Rep}\left(\mathbf{S}_{\zeta}\right)$.

Proof. We must check that $R$ identifies the tensor product $\dot{\otimes}$ with the induction (3.8). First, fix two projective objects $X \in \mathcal{O}\left(\mathfrak{S}_{m^{\prime}}\right)$ and $Y \in \mathcal{O}\left(\mathfrak{S}_{m^{\prime \prime}}\right)$. We have

$$
\begin{aligned}
\Phi^{*}(R(X) \dot{\otimes} R(Y)) & ={ }^{\mathbf{H}} \operatorname{Ind}_{m^{\prime}, m^{\prime \prime}}\left(\Phi^{*} R(X) \otimes \Phi^{*} R(Y)\right) \\
& ={ }^{\mathbf{H}} \operatorname{Ind}_{m^{\prime}, m^{\prime \prime}}(\mathrm{KZ}(X) \otimes \mathrm{KZ}(Y)) \\
& =\mathrm{KZ}\left({ }^{\mathcal{O}} \operatorname{Ind}_{m^{\prime}, m^{\prime \prime}}(X \otimes Y)\right) \\
& =\Phi^{*} R\left({ }^{\mathcal{O}} \operatorname{Ind}_{m^{\prime}, m^{\prime \prime}}(X \otimes Y)\right) .
\end{aligned}
$$

The first equality follows from Corollary B.4 the second one and the fourth one come from $\mathrm{KZ}=\Phi^{*} \circ R$, and the third one is the commutation of $\mathrm{KZ}$ and the induction functors; see [33. Since the modules $R(X) \dot{\otimes} R(Y)$ and $R\left({ }^{\mathcal{O}} \operatorname{Ind}_{m^{\prime}, m^{\prime \prime}}(X \otimes\right.$ $Y)$ ) are projective, and since $\Phi^{*}$ is fully faithful on projectives we get that

$$
R(X) \dot{\otimes} R(Y)=R\left({ }^{\mathcal{O}} \operatorname{Ind}_{m^{\prime}, m^{\prime \prime}}(X \otimes Y)\right) .
$$

Now, since the functors (B.6), (3.8) are exact and coincide on projective objects, and since the category $\mathcal{O}\left(\mathfrak{S}_{m}\right)$ has enough projectives, the proposition is proved.

3.9. The categorification of $\tilde{\mathfrak{s l}}_{m}$. Recall that $Z\left(\mathcal{O}\left(\Gamma_{n}\right)\right)$ is the center of the category $\mathcal{O}\left(\Gamma_{n}\right)$. Let $D_{n}(z)$ be the polynomial in $Z\left(\mathcal{O}\left(\Gamma_{n}\right)\right)[z]$ defined in [33, sec. 4.2]. For any $a \in \mathbb{C}(z)$ the projection to the generalized eigenspace of $D_{n}(z)$ with the eigenvalue $a$ yields an exact endofunctor $Q_{n, a}$ of $\mathcal{O}\left(\Gamma_{n}\right)$. Next, consider the point

$$
b_{n}=(0,0, \ldots, 0,1) \in \mathfrak{h}, \quad \mathfrak{h}=\mathbb{C}^{n} .
$$

The induction and the restriction relative to $b_{n}$ yield functors

$$
{ }^{\mathcal{O}} \operatorname{Ind}_{n}: \mathcal{O}\left(\Gamma_{n-1}\right) \rightarrow \mathcal{O}\left(\Gamma_{n}\right), \quad{ }^{\mathcal{O}} \operatorname{Res}_{n}: \mathcal{O}\left(\Gamma_{n}\right) \rightarrow \mathcal{O}\left(\Gamma_{n-1}\right) .
$$

Definition 3.2 ([33, sec. 4.2]). The $q$-restiction and the $q$-induction functors

$$
e_{q}: \mathcal{O}\left(\Gamma_{n}\right) \rightarrow \mathcal{O}\left(\Gamma_{n-1}\right), \quad f_{q}: \mathcal{O}\left(\Gamma_{n-1}\right) \rightarrow \mathcal{O}\left(\Gamma_{n}\right), \quad q=0,1, \ldots, m-1
$$

are given by

$$
\begin{aligned}
e_{q} & =\bigoplus_{a \in \mathbb{C}(z)} Q_{n-1, a /\left(z-\zeta^{q}\right)} \circ{ }^{\mathcal{O}} \operatorname{Res}_{n} \circ Q_{n, a}, \\
f_{q} & =\bigoplus_{a \in \mathbb{C}(z)} Q_{n, a\left(z-\zeta^{q}\right)}{ }^{\mathcal{O}} \operatorname{Ind}_{n} \circ Q_{n-1, a} .
\end{aligned}
$$


We'll abbreviate

$$
E=e_{0} \oplus e_{1} \oplus \cdots \oplus e_{m-1}, \quad F=f_{0} \oplus f_{1} \oplus \cdots \oplus f_{m-1} .
$$

Following [33, sec. 6.3], for $L \in \operatorname{Irr}(\mathcal{O}(\Gamma))$ we set

$$
\tilde{e}_{q}(L)=\operatorname{top}\left(e_{q}(L)\right), \quad \tilde{f}_{q}(L)=\operatorname{soc}\left(f_{q}(L)\right), \quad \tilde{e}_{q}(0)=\tilde{f}_{q}(0)=0 .
$$

Now, for each $n$ we choose the parameters of $H\left(\Gamma_{n}\right)$ in the following way:

$$
h=-1 / m, \quad h_{p}=\left(s_{p+1}-s_{p}\right) / m, \quad s_{q} \in \mathbb{Z}, \quad p \neq 0 .
$$

The following hypothesis is important for the rest of the paper:

$$
\text { from now on we'll always assume that } m>1 \text {. }
$$

The $\mathbb{C}$-vector space $[\mathcal{O}(\Gamma)]$ is canonically isomorphic to the level $\ell$ Fock space $\mathcal{F}_{m, \ell}^{(s)}$ associated with the $\ell$-charge $s=\left(s_{p}\right)$; see (5.20) below for details. The latter is equipped with an integrable representation of $\widetilde{\mathfrak{s l}}_{m}$ of level $\ell$; see Section 4.6 below.

Proposition 3.3. (a) The functors $e_{q}, f_{q}$ are exact and biadjoint.

(b) We have $E={ }^{\mathcal{O}} \operatorname{Res}_{n}$ and $F={ }^{\mathcal{O}} \operatorname{Ind}_{n}$.

(c) For $M \in \mathcal{O}\left(\Gamma_{n}\right)$ we have $E(M)=0$ (resp. $F(M)=0$ ) iff $E(L)=0$ (resp. $F(L)=0$ ) for any constituent $L$ of $M$.

(d) The operators $e_{q}, f_{q}$ equip $[\mathcal{O}(\Gamma)]$ with a representation of $\widehat{\mathfrak{s l}}_{m}$ which is isomorphic via the map (5.20) to $\mathcal{F}_{m, \ell}^{(s)}$.

(e) The tuple $\left(\operatorname{Irr}(\mathcal{O}(\Gamma)), \tilde{e}_{q}, \tilde{f}_{q}\right)$ has a crystal structure. In particular, for $L, L^{\prime} \in$ $\operatorname{Irr}(\mathcal{O}(\Gamma))$ we have $\tilde{e}_{q}(L), \tilde{f}_{q}(L) \in \operatorname{Irr}(\mathcal{O}(\Gamma)) \sqcup\{0\}$, and $\tilde{e}_{q}(L)=L^{\prime}$ if and only if $\tilde{f}_{q}\left(L^{\prime}\right)=L$.

Proof. Parts $(a),(b)$ follow from [33, prop. 4.4], part $(e)$ is contained in [33, thm. 6.3], part $(c)$ is obvious, and part $(d)$ is [33, cor. 4.5].

3.10. The filtration of $\left[\mathcal{O}\left(\Gamma_{n}\right)\right]$ by the support. Fix a positive integer $n$. Assume that $\ell \geqslant 2$. In this section we consider the tautological action of $\Gamma_{n}$ on $\mathbb{C}^{n}$. For an integer $l \geqslant 0$ and a composition $\nu$ such that $l+|\nu| \leqslant n$ we abbreviate $X_{l, \nu}^{\circ}=X_{W, \mathfrak{h}}^{\circ}$ and $X_{l, \nu}=X_{W, \mathfrak{h}}$, where $W=\Gamma_{l, \nu}$. If $\nu=\left(m^{j}\right)$ for some integer $j \geqslant 0$ such that $l+j m \leqslant n$ we write

$$
X_{l, j}^{\circ}=X_{l, \nu}^{\circ}, \quad X_{l, j}=X_{l, \nu} .
$$

Therefore $X_{l, j}$ is the set of the points in $\mathbb{C}^{n}$ with $l$ coordinates equal to zero and $j$ collections of $m$ coordinates which differ from each other by $\ell$-th roots of one. To avoid confusion we may write $X_{l, j, \mathbb{C}^{n}}=X_{l, j}$. Unless specified otherwise, for $l, j, m, n$ as above we'll set

$$
i=n-l-j m \text {. }
$$

Definition 3.4. For $i, j \geqslant 0$ we set

$$
\operatorname{Irr}\left(\mathcal{O}\left(\Gamma_{n}\right)\right)_{i, j}=\left\{L \in \operatorname{Irr}\left(\mathcal{O}\left(\Gamma_{n}\right)\right): \operatorname{Supp}(L)=X_{l, j}\right\} .
$$

Definition 3.5. For $i, j \geqslant 0$ let $F_{i, j}\left(\Gamma_{n}\right)$ be the $\mathbb{C}$-vector subspace of $\left[\mathcal{O}\left(\Gamma_{n}\right)\right]$ spanned by the classes of the modules whose support is contained in $X_{l, j}$, with $l$ as in (3.10). If $i<0$ or $j<0$ we write $F_{i, j}\left(\Gamma_{n}\right)=0$.

Definition 3.6. We define a partial order on the set of pairs of nonnegative integers $(i, j)$ such that $i+j m \leqslant n$ given by $\left(i^{\prime}, j^{\prime}\right) \leqslant(i, j)$ if and only if $X_{l^{\prime}, j^{\prime}} \subset X_{l, j}$, where $l=n-i-j m$ and $l^{\prime}=n-i^{\prime}-j^{\prime} m$. 
Since the support of a module is the union of the supports of all of its constituents, the $\mathbb{C}$-vector space $F_{i, j}\left(\Gamma_{n}\right)$ is spanned by the classes of the modules in $\operatorname{Irr}\left(\mathcal{O}\left(\Gamma_{n}\right)\right)$ whose support is contained in $X_{l, j}$, or, equivalently, $F_{i, j}\left(\Gamma_{n}\right)$ is spanned by the classes of the modules in

$$
\bigcup_{\left(i^{\prime}, j^{\prime}\right) \leqslant(i, j)} \operatorname{Irr}\left(\mathcal{O}\left(\Gamma_{n}\right)\right)_{i^{\prime}, j^{\prime}} .
$$

Remark 3.7. We have $\bigcup_{i, j} F_{i, j}\left(\Gamma_{n}\right)=\left[\mathcal{O}\left(\Gamma_{n}\right)\right]$. Indeed, for $L \in \operatorname{Irr}\left(\mathcal{O}\left(\Gamma_{n}\right)\right)$ we have $\operatorname{Supp}(L)=X_{l, \nu}$ for some $l, \nu$; see Section 2.2. For $b \in X_{l, \nu}^{\circ}$ the $H\left(\Gamma_{l, \nu}\right)$-module $\operatorname{Res}_{b}(L)$ is finite dimensional. Thus, since the parameter $h$ of $H\left(\Gamma_{l, \nu}\right)$ is equal to $-1 / m$ the parts of $\nu$ are all equal to $m$. Hence we have $\operatorname{Supp}(L)=X_{l, j}$ for some $l, j$ as above.

The subspaces $F_{i, j}\left(\Gamma_{n}\right)$ give a filtration of $\left[\mathcal{O}\left(\Gamma_{n}\right)\right]$. Consider the associated graded $\mathbb{C}$-vector space

$$
\operatorname{gr}\left(\Gamma_{n}\right)=\bigoplus_{i, j} \operatorname{gr}_{i, j}\left(\Gamma_{n}\right)
$$

The images by the canonical projection $F_{i, j}\left(\Gamma_{n}\right) \rightarrow \operatorname{gr}_{i, j}\left(\Gamma_{n}\right)$ of the classes of the modules in $\operatorname{Irr}\left(\mathcal{O}\left(\Gamma_{n}\right)\right)_{i, j}$ form a basis of the $\mathbb{C}$-vector space $\operatorname{gr}_{i, j}\left(\Gamma_{n}\right)$. So we may regard $\operatorname{gr}_{i, j}\left(\Gamma_{n}\right)$ as the subspace of $\left[\mathcal{O}\left(\Gamma_{n}\right)\right]$ spanned by $\operatorname{Irr}\left(\mathcal{O}\left(\Gamma_{n}\right)\right)_{i, j}$. We'll abbreviate

$$
\begin{array}{cc}
F_{i, \bullet}\left(\Gamma_{n}\right)=\sum_{j} F_{i, j}\left(\Gamma_{n}\right), & F_{\bullet, j}\left(\Gamma_{n}\right)=\sum_{i} F_{i, j}\left(\Gamma_{n}\right), \\
\operatorname{gr}_{i, \bullet}\left(\Gamma_{n}\right)=\bigoplus_{j} \operatorname{gr}_{i, j}\left(\Gamma_{n}\right), & \operatorname{gr}_{\bullet}, j
\end{array}
$$

Now, let us study the filtration of $\left[\mathcal{O}\left(\Gamma_{n}\right)\right]$ in detail. The subgroup $\Gamma_{l,\left(m^{j}\right)}$ of $\Gamma_{n}$ is contained in the subgroups $\Gamma_{l+1,\left(m^{j}\right)}, \Gamma_{l,\left(m^{j+1}\right)}$ and $\Gamma_{l+m,\left(m^{j-1}\right)}$ (up to conjugation by an element of $\Gamma_{n}$ ) whenever such subgroups exist. Thus we have the inclusions

$$
\begin{gathered}
X_{l+1, j}, X_{l, j+1}, X_{l+m, j-1} \subset X_{l, j}, \\
F_{i-1, j}\left(\Gamma_{n}\right), F_{i-m, j+1}\left(\Gamma_{n}\right), F_{i, j-1}\left(\Gamma_{n}\right) \subset F_{i, j}\left(\Gamma_{n}\right) .
\end{gathered}
$$

Proposition 3.8. (a) We have

$$
X_{l^{\prime}, j^{\prime}} \subsetneq X_{l, j} \Longleftrightarrow X_{l^{\prime}, j^{\prime}} \subset X_{l+1, j} \cup X_{l, j+1} \cup X_{l+m, j-1} .
$$

(b) We have an isomorphism of $\mathbb{C}$-vector spaces

$$
g r_{i, j}\left(\Gamma_{n}\right)=F_{i, j}\left(\Gamma_{n}\right) /\left(F_{i-1, j}\left(\Gamma_{n}\right)+F_{i-m, j+1}\left(\Gamma_{n}\right)+F_{i, j-1}\left(\Gamma_{n}\right)\right) .
$$

Proof. First we prove $(a)$. Recall that $X_{l, j}$ is the set of the points in $\mathbb{C}^{n}$ with $l$ coordinates equal to zero and $j$ collections of $m$ coordinates which differ from each other by $\ell$-th roots of one. Therefore we have

$$
X_{l^{\prime}, j^{\prime}} \subset X_{l, j} \Longleftrightarrow i-i^{\prime} \geqslant \max \left(0,\left(j^{\prime}-j\right) m\right) .
$$

In particular this inclusion implies that $l^{\prime} \geqslant l$. We must prove that

$$
X_{l^{\prime}, j^{\prime}} \subsetneq X_{l, j} \Rightarrow X_{l^{\prime}, j^{\prime}} \subset X_{l+1, j} \cup X_{l, j+1} \cup X_{l+m, j-1} .
$$

First, assume that $l^{\prime}=l$. Since $X_{l^{\prime}, j^{\prime}} \subsetneq X_{l, j}$ we have $i>i^{\prime}$. Then (3.10) implies that $i-i^{\prime}=\left(j^{\prime}-j\right) m$, hence that $j^{\prime}>j$ and $i-i^{\prime} \geqslant m$. So $i-i^{\prime} \geqslant \max \left(m,\left(j^{\prime}-j\right) m\right)$, and (3.11) implies that $X_{l^{\prime}, j^{\prime}} \subset X_{l, j+1}$. 
Next, assume that $l+m>l^{\prime}>l$. Since $X_{l^{\prime}, j^{\prime}} \subset X_{l, j}$ we have $i \geqslant i^{\prime}$. Further (3.10) implies that $i-i^{\prime}>\left(j^{\prime}-j\right) m$ and $i^{\prime}-i>\left(j-j^{\prime}-1\right) m$. Thus $i \geqslant i^{\prime}$ implies indeed that $i>i^{\prime}$ and $j^{\prime} \geqslant j$. So $i-1-i^{\prime} \geqslant \max \left(0,\left(j^{\prime}-j\right) m\right)$, and (3.11) implies that $X_{l^{\prime}, j^{\prime}} \subset X_{l+1, j}$.

Finally, assume that $l^{\prime} \geqslant l+m$. Since $X_{l^{\prime}, j^{\prime}} \subset X_{l, j}$ we have $i \geqslant i^{\prime}$. Further (3.10) implies that $i-i^{\prime} \geqslant\left(j^{\prime}-j+1\right) m$. So $i-i^{\prime} \geqslant \max \left(0,\left(j^{\prime}-j+1\right) m\right)$, and (3.11) implies that $X_{l^{\prime}, j^{\prime}} \subset X_{l+m, j-1}$.

Part $(b)$ is a consequence of $(a)$ and of the definition of the filtration on $\left[\mathcal{O}\left(\Gamma_{n}\right)\right]$.

Remark 3.9. The sets $X_{l+1, j}, X_{l, j+1}, X_{l+m, j-1}$ do not contain each other. Indeed, the variety $X_{l, j}$ has the dimension $i+j$. Thus the codimensions of $X_{l+1, j}, X_{l, j+1}$, $X_{l+m, j-1}$ in $X_{l, j}$ are $1, m-1,1$ respectively. However, since a point in $X_{l, j+1}^{\circ}$ has only $l$ coordinates equal to 0 , we have $X_{l, j+1} \not \subset X_{l+1, j}$ and $X_{l, j+1} \not \subset X_{l+m, j-1}$.

Remark 3.10. We have $F_{\bullet, 0}\left(\Gamma_{n}\right)=\left[\mathcal{O}\left(\Gamma_{n}\right)\right]$, because $(i, j) \leqslant(i+j m, 0)$.

Remark 3.11. We have $\left(i^{\prime}, j^{\prime}\right) \leqslant(0, j)$ if and only if $i^{\prime}=0$ and $j^{\prime} \leqslant j$.

Remark 3.12. Consider the set

$$
F_{i, j}\left(\Gamma_{n}\right)^{\circ}=F_{i, j}\left(\Gamma_{n}\right) \backslash\left(F_{i-1, j}\left(\Gamma_{n}\right)+F_{i-m, j+1}\left(\Gamma_{n}\right)+F_{i, j-1}\left(\Gamma_{n}\right)\right) .
$$

For $L \in \operatorname{Irr}\left(\mathcal{O}\left(\Gamma_{n}\right)\right)$, by Proposition 3.8 and Remark 3.7 we have

$$
\begin{aligned}
{[L] \in F_{i, j}\left(\Gamma_{n}\right)^{\circ} } & \Longleftrightarrow \operatorname{Supp}(L)=X_{l, j} \\
& \Longleftrightarrow L \in \operatorname{Irr}\left(\mathcal{O}\left(\Gamma_{n}\right)\right)_{i, j} .
\end{aligned}
$$

Remark 3.13. A representation is finite dimensional if and only if its support is zero. Thus $\operatorname{Irr}\left(\mathcal{O}\left(\Gamma_{n}\right)\right)_{0,0}$ is the set of isomorphism classes of finite-dimensional modules in $\mathcal{O}\left(\Gamma_{n}\right)$. Note that $(0,0) \leqslant(i, j)$ for all $(i, j)$.

Remark 3.14. If $\ell=1$, then, by Remark 2.1 and Section 3.2 we have $\mathcal{O}\left(\mathfrak{S}_{n}\right)=$ $\mathcal{O}\left(\mathfrak{S}_{n}, \mathbb{C}_{0}^{n}\right)$. For an integer $j \geqslant 0$ we set $X_{j}=X_{\mathfrak{S}_{m}^{j}, \mathbb{C}_{0}^{n}}$; i.e., $X_{j}$ is the set of the points in $\mathbb{C}_{0}^{n}$ with $j$ collections of $m$ equal coordinates. Then, we set $i=n-j m$ and the results of this section extend in the obvious way. In particular, we have

$$
X_{j^{\prime}} \subset X_{j} \Longleftrightarrow j^{\prime} \geqslant j, \quad X_{j^{\prime}} \subsetneq X_{j} \Longleftrightarrow X_{j^{\prime}} \subset X_{j+1} .
$$

Remark 3.15. For $\lambda \in \mathcal{P}_{r}, r \geqslant 1$, the support of the module $L_{m \lambda} \in \operatorname{Irr}\left(\mathcal{O}\left(\mathfrak{S}_{m r}\right)\right)$ is

$$
\operatorname{Supp}\left(L_{m \lambda}\right)=X_{\mathfrak{S}_{m}^{r}, \mathbb{C}_{0}^{m r}} .
$$

Indeed, formula (5.16) below and Proposition 2.7 imply that

$$
\operatorname{Supp}\left(L_{m \lambda}\right) \subset \operatorname{Supp}\left({ }^{\mathcal{O}} \operatorname{Ind}_{\left(m^{r}\right)}\left(L_{(m)}^{\otimes r}\right)\right)=X_{\mathfrak{S}_{m}^{r}, \mathbb{C}_{0}^{m r}} .
$$

Next, by Remark 3.7 there is $j=0,1, \ldots, r$ such that

$$
\operatorname{Supp}\left(L_{m \lambda}\right)=X_{\mathfrak{S}_{m}^{j}, \mathbb{C}_{0}^{m r}} .
$$

Finally the inclusion $X_{\mathfrak{S}_{m}^{j}, \mathbb{C}_{0}^{m r}} \subset X_{\mathfrak{S}_{m}^{r}, \mathbb{C}_{0}^{m r}}$ implies that $j=r$ by Remark 3.14 . Note that the equality (3.12) also follows from the work of Wilcox [37. 
3.11. The action of $E, F$ on the filtration. Let $E, F$ denote the $\mathbb{C}$-linear operators on $[\mathcal{O}(\Gamma)]$ induced by the exact functors $E, F$. Recall that the parameters of $H(\Gamma)$ are chosen as in (3.9).

Proposition 3.16. Let $L \in \operatorname{Irr}\left(\mathcal{O}\left(\Gamma_{n}\right)\right)_{i, j}$ and $l=n-i-m j$.

(a) We have $\operatorname{Supp}(F(L))=X_{l, j, \mathbb{C}^{n+1}}$.

(b) We have $E(L)=0$ iff $i=0$. We have $\operatorname{Supp}(E(L))=X_{l, j, \mathbb{C}^{n-1}}$ if $i>0$.

Proof. Recall that

$$
\operatorname{Supp}(L)=X_{l, j}=X_{l, j, \mathbb{C}^{n}}, \quad E(L)={ }^{\mathcal{O}} \operatorname{Res}_{n}(L), \quad F(L)={ }^{\mathcal{O}} \operatorname{Ind}_{n}(L) .
$$

Thus by Proposition 2.2 we have $E(L)=0$ iff $b_{n} \notin X_{l, j}$. Since $m>1$ the definition of the stratum $X_{l, j}$ in Section 3.10 shows that $b_{n} \notin X_{l, j}$ iff $i=0$. Now, assume that $i>0$. Then $l+m j \leqslant n-1$, and Proposition 2.2 yields

$$
\operatorname{Supp}(E(L))=\bigcup_{W} X_{W, \mathbb{C}^{n-1}},
$$

where $W$ runs over the parabolic subgroups of $\Gamma_{n-1}$ which are $\Gamma_{n}$-conjugate to $\Gamma_{l,\left(m^{j}\right)}$ (inside the group $\left.\Gamma_{n}\right)$. We claim that a subgroup $W \subset \Gamma_{n-1}$ as above is $\Gamma_{n-1}$-conjugate to $\Gamma_{l,\left(m^{j}\right)}$ (inside the group $\left.\Gamma_{n-1}\right)$. Therefore, we have

$$
\operatorname{Supp}(E(L))=X_{l, j, \mathbb{C}^{n-1}} .
$$

Indeed, fix $b^{\prime} \in \mathbb{C}^{n-1}$ such that $W=\left(\Gamma_{n-1}\right)_{b^{\prime}}$. For $b=\left(b^{\prime}, z\right)$ with $z \in \mathbb{C}$ generic we have $\left(\Gamma_{n}\right)_{b}=W$, where $W$ is regarded as a subgroup of $\Gamma_{n}$ via the obvious inclusion $\Gamma_{n-1} \subset \Gamma_{n}$. Since $W$ is $\Gamma_{n}$-conjugate to $\Gamma_{l,\left(m^{j}\right)}$, there is an element $g \in \Gamma_{n}$ such that the first $l$ coordinates of $g(b)$ are 0 , the next $m j$ ones consist of $j$ collections of $m$ coordinates which are proportional to each other with a ratio given by an $\ell$-th root of one, and the last $i$ coordinates of $g(b)$ are in generic position. We'll abbreviate

$$
g(b) \in 0^{l}(m)^{j} *^{i} .
$$

Since $z$ is generic it is taken by $g$ to one of the coordinates of $g(b)$ in the packet $*^{i}$. Composing $g$ by an appropriate reflection in $\mathfrak{S}_{n}$ we get an element $g^{\prime} \in \Gamma_{n-1}$ such that

$$
g^{\prime}(b)=\left(g^{\prime}\left(b^{\prime}\right), z\right) \in 0^{l}(m)^{j} *^{i} .
$$

Thus we also have

$$
g^{\prime}\left(b^{\prime}\right) \in 0^{l}(m)^{j} *^{i-1} .
$$

This implies the claim. Hence, we have

$$
\operatorname{Supp}(E(L))=X_{l, j, \mathbb{C}^{n-1}} .
$$

Finally, since $\operatorname{Supp}(L)=X_{l, j, \mathbb{C}^{n}}$, Proposition 2.7 implies that

$$
\operatorname{Supp}(F(L))=X_{l, j, \mathbb{C}^{n+1}} .
$$

Corollary 3.17. (a) We have $E\left(F_{i, j}\left(\Gamma_{n}\right)\right) \subset F_{i-1, j}\left(\Gamma_{n-1}\right)$. If $i \neq 0$ we also have $E\left(F_{i, j}\left(\Gamma_{n}\right)^{\circ}\right) \subset F_{i-1, j}\left(\Gamma_{n-1}\right)^{\circ}$.

(b) For $M \in \mathcal{O}\left(\Gamma_{n}\right)$ with $[M] \in F_{i, j}\left(\Gamma_{n}\right)^{\circ}$ we have $E([M])=0$ iff $i=0$.

(c) We have $F\left(F_{i, j}\left(\Gamma_{n}\right)\right) \subset F_{i+1, j}\left(\Gamma_{n+1}\right)$ and $F\left(F_{i, j}\left(\Gamma_{n}\right)^{\circ}\right) \subset F_{i+1, j}\left(\Gamma_{n+1}\right)^{\circ}$. 
Proof. First, let $L \in \operatorname{Irr}\left(\mathcal{O}\left(\Gamma_{n}\right)\right)$ with $[L] \in F_{i, j}\left(\Gamma_{n}\right)$. Thus $L \in \operatorname{Irr}(\mathcal{O}(\Gamma))_{i^{\prime}, j^{\prime}}$ with $\left(i^{\prime}, j^{\prime}\right) \leqslant(i, j)$. Proposition 3.16 yields

$$
\operatorname{Supp}(F(L))=X_{l^{\prime}, j^{\prime}, \mathbb{C}^{n+1}}, \quad \operatorname{Supp}(E(L))=X_{l^{\prime}, j^{\prime}, \mathbb{C}^{n-1}} \text { if } i^{\prime} \neq 0 .
$$

Hence we have $F([L]) \in F_{i+1, j}\left(\Gamma_{n+1}\right)$ and $E([L]) \in F_{i-1, j}\left(\Gamma_{n-1}\right)$. Part $(b)$ follows from Proposition 3.16 and Remarks 3.11, 3.12, Part $(c)$ follows from Proposition 3.16 and Remark 3.12. The second part of $(a)$ follows from Proposition 3.16 and Remark 3.12

Corollary 3.18. Let $L \in \operatorname{Irr}\left(\mathcal{O}\left(\Gamma_{n}\right)\right)_{i, j}$.

(a) If $\tilde{e}_{q}(L) \neq 0$, then $\tilde{e}_{q}(L) \in \operatorname{Irr}\left(\mathcal{O}\left(\Gamma_{n-1}\right)\right)_{i-1, j}$.

(b) If $\tilde{f}_{q}(L) \neq 0$, then $\tilde{f}_{q}(L) \in \operatorname{Irr}\left(\mathcal{O}\left(\Gamma_{n+1}\right)\right)_{i+1, j}$.

Proof. Set $L^{\prime}=\tilde{e}_{q}(L)$. Assume that $L^{\prime} \neq 0$. By Proposition 3.3 we have

$$
L^{\prime} \in \operatorname{Irr}\left(\mathcal{O}\left(\Gamma_{n-1}\right)\right), \quad \tilde{f}_{q}\left(L^{\prime}\right)=L .
$$

Next, since $L \in \operatorname{Irr}(\mathcal{O}(\Gamma))_{i, j}$ and since $\tilde{e}_{q}(L)$ is a constituent of $E(L)$, we have $\left[L^{\prime}\right] \in F_{i-1, j}\left(\Gamma_{n-1}\right)$ by Corollary 3.17 We must prove that $\left[L^{\prime}\right] \in F_{i-1, j}\left(\Gamma_{n-1}\right)^{\circ}$. If this is false, then we have $\left[L^{\prime}\right] \in F_{i^{\prime}, j^{\prime}}\left(\Gamma_{n-1}\right)$ with

$$
\left(i^{\prime}, j^{\prime}\right)=(i-2, j),(i-m-1, j+1),(i-1, j-1) .
$$

Thus, since $\tilde{f}_{q}\left(L^{\prime}\right)$ is a constituent of $F\left(L^{\prime}\right)$, by Corollary 3.17 we have

$$
[L] \in \operatorname{gr}_{i, j}\left(\Gamma_{n}\right) \cap F_{i^{\prime}+1, j^{\prime}}\left(\Gamma_{n}\right) .
$$

Therefore (3.11) yields $i^{\prime}+1 \geqslant i$, so $i^{\prime}=i-1$ and $j^{\prime}=j-1$. So, applying (3.11) once again we get a contradiction with (3.13). This proves $(a)$. The proof of $(b)$ is similar.

Corollary 3.19. (a) For $x \in[\mathcal{O}(\Gamma)]$ we have

$$
\left(e_{q}(x)=0, \forall q=0,1, \ldots, m-1\right) \Longleftrightarrow x \in F_{0, \bullet}(\Gamma) .
$$

(b) For $M \in \mathcal{O}(\Gamma)$ we have

$$
E(M)=0 \Longleftrightarrow E([M])=0 \Longleftrightarrow[M] \in F_{0, \bullet}(\Gamma) .
$$

(c) The space $F_{0, \bullet}(\Gamma)$ is spanned by the set

$$
\begin{aligned}
\left\{[L]: L \in \operatorname{Irr}(\mathcal{O}(\Gamma))_{0, \bullet}\right\} & =\{[L]: L \in \operatorname{Irr}(\mathcal{O}(\Gamma)), E(L)=0\} \\
& =\left\{[L]: L \in \operatorname{Irr}(\mathcal{O}(\Gamma)), \tilde{e}_{q}(L)=0, \forall q=0,1, \ldots, m-1\right\} .
\end{aligned}
$$

Proof. For $x \in[\mathcal{O}(\Gamma)]$ we write $x=\sum_{L} x_{L}[L]$, where $L$ runs over the $\operatorname{set} \operatorname{Irr}(\mathcal{O}(\Gamma))$. By [33, lem. 6.1, prop. 6.2], for each $q$ we have

$$
e_{q}(x)=0 \Longleftrightarrow x_{L}=0 \text { if } e_{q}([L]) \neq 0 .
$$

Thus the $\mathbb{C}$-vector space

$$
\left\{x \in[\mathcal{O}(\Gamma)]: e_{q}(x)=0, \forall q=0,1, \ldots, m-1\right\}
$$

is spanned by the classes of the simple modules $L$ such that $e_{q}([L])=0$ for all $q=0,1, \ldots, m-1$. Then, apply Corollary 3.17. This proves $(a)$. Parts $(b),(c)$ are obvious. Note that

$$
\tilde{e}_{q}(L)=0, \forall q \Longleftrightarrow e_{q}(L)=0, \forall q,
$$

because a nonzero finitely generated module has a nonzero top. 


\section{THe Fock SPACE}

From now on we'll abbreviate

$$
R(\mathfrak{S})=\bigoplus_{n \geqslant 0}\left[\operatorname{Rep}\left(\mathbb{C S}_{n}\right)\right], \quad R(\Gamma)=\bigoplus_{n \geqslant 0}\left[\operatorname{Rep}\left(\mathbb{C} \Gamma_{n}\right)\right]
$$

4.1. The Hopf $\mathbb{C}$-algebra $\lambda$. This section and the following one are reminders on symmetric functions and the Heisenberg algebra. First, recall that the $\mathbb{C}$-vector space $R(\mathfrak{S})$ is identified with the $\mathbb{C}$-vector space of symmetric functions

$$
\boldsymbol{\Lambda}=\mathbb{C}\left[x_{1}, x_{2}, \ldots\right]^{\mathfrak{S}_{\infty}}
$$

via the characteristic map [27, chap. I]

$$
\operatorname{ch}: R(\mathfrak{S}) \rightarrow \boldsymbol{\Lambda} \text {. }
$$

The map ch intertwines the induction/restriction in $R(\mathfrak{S})$ with the multiplication/comultiplication in $\boldsymbol{\Lambda}$. More precisely, for each $m, n \geqslant 0$ the restriction yields a linear map

$$
\operatorname{Res}_{n, m}:\left[\operatorname{Rep}\left(\mathbb{C S}_{n+m}\right)\right] \rightarrow\left[\operatorname{Rep}\left(\mathbb{C S}_{n}\right)\right] \otimes\left[\operatorname{Rep}\left(\mathbb{C S}_{n}\right)\right] .
$$

Then, under ch, the sum $\bigoplus_{n, m} \operatorname{Res}_{n, m}$ is identified with the coproduct of $\boldsymbol{\Lambda}$. The map ch takes the class of the simple module $\bar{L}_{\lambda}$ to the Schur function $S_{\lambda}$ for each $\lambda \in \mathcal{P}$. The power sum polynomials are given by

$$
P_{\lambda}=P_{\lambda_{1}} P_{\lambda_{2}} \ldots, \quad P_{r}=\sum_{i} x_{i}^{r}, \quad P_{0}=1, \quad \lambda \in \mathcal{P}, \quad r>0 .
$$

We equip the $\mathbb{C}$-vector space $\boldsymbol{\Lambda}$ with the level 1 action of $\widehat{\mathfrak{s l}}_{m}$ given by

$$
e_{q}\left(S_{\lambda}\right)=\sum_{\nu} S_{\nu}, \quad f_{q}\left(S_{\lambda}\right)=\sum_{\mu} S_{\mu}, \quad q=0, \ldots, m-1,
$$

where $\nu$ (resp. $\mu$ ) runs through all partitions obtained from $\lambda \in \mathcal{P}$ by removing (resp. adding) a node of content $q \bmod m$. We equip $\boldsymbol{\Lambda}$ with the symmetric bilinear form such that the Schur functions form an orthonormal basis. The operators $e_{q}$, $f_{q}$ are adjoint to each other for this pairing.

4.2. The Heisenberg algebra. The Heisenberg algebra is the Lie algebra $\mathfrak{H}$ spanned by the elements $\mathbf{1}$ and $b_{r}, b_{r}^{\prime}, r>0$, satisfying the following relations:

$$
\left[b_{r}^{\prime}, b_{s}^{\prime}\right]=\left[b_{r}, b_{s}\right]=0, \quad\left[b_{r}^{\prime}, b_{s}\right]=r \mathbf{1} \delta_{r, s}, \quad r, s>0 .
$$

Let $U(\mathfrak{H})$ be the enveloping algebra of $\mathfrak{H}$, and let $U^{-}(\mathfrak{H}) \subset U(\mathfrak{H})$ be the subalgebra generated by the elements $b_{r}$ with $r>0$. Write $U^{-}(\mathfrak{H})_{r}$ for the subspace of $U^{-}(\mathfrak{H})$ spanned by the monomials $b_{r_{1}} b_{r_{2}} \cdots$ with $\sum_{i} r_{i}=r$. For $\lambda \in \mathcal{P}$ and $f \in \boldsymbol{\Lambda}$ we consider the following elements in $U(\mathfrak{H})$ :

$$
\begin{aligned}
& b_{\lambda}=b_{\lambda_{1}} b_{\lambda_{2}} \cdots, b_{\lambda}^{\prime}=b_{\lambda_{1}}^{\prime} b_{\lambda_{2}}^{\prime} \cdots, \\
& b_{f}=\sum_{\lambda \in \mathcal{P}} z_{\lambda}^{-1}\left\langle P_{\lambda}, f\right\rangle b_{\lambda}, \quad b_{f}^{\prime}=\sum_{\lambda \in \mathcal{P}} z_{\lambda}^{-1}\left\langle P_{\lambda}, f\right\rangle b_{\lambda}^{\prime},
\end{aligned}
$$

where $z_{\lambda}$ is as in (3.1). For any integer $\ell$ we can equip $\boldsymbol{\Lambda}$ with the level $\ell$ action of $\mathfrak{H}$ such that $b_{r}$ acts by multiplication by $\ell P_{r}$ and $b_{r}^{\prime}$ acts by $r \partial / \partial_{P_{r}}$ for $r>0$. The operators $b_{r}, b_{r}^{\prime}$ are adjoint to each other for the pairing on $\boldsymbol{\Lambda}$ introduced in 
Section 4.1. Further, they commute with the $\widehat{\mathfrak{s l}}_{m}$-action in (4.1); see, e.g., 35, prop. 4.6]. We write $V_{\ell}^{\mathfrak{H}}=\Lambda$ regarded as a level $\ell$ module of $\mathfrak{H}$. Consider the Casimir operator

$$
\partial=\frac{1}{\ell} \sum_{r \geqslant 1} b_{r} b_{r}^{\prime} .
$$

To avoid any confusion we may also call it the level $\ell$ Casimir operator. This formal sum defines a diagonalisable $\mathbb{C}$-linear operator on $V_{\ell}^{\mathfrak{H}}$ such that

$$
\left[\partial, b_{r}\right]=r b_{r}, \quad\left[\partial, b_{r}^{\prime}\right]=-r b_{r}^{\prime} .
$$

For each integer $j$ let $V_{\ell}^{\mathfrak{H}}[j] \subset V_{\ell}^{\mathfrak{H}}$ be the eigenspace of $\partial$ associated with the eigenvalue $j$. Below, we'll equip $\boldsymbol{\Lambda}$ with the $\mathfrak{H}$-action of level 1 , i.e., we'll identify $\boldsymbol{\Lambda}=V_{1}^{\mathfrak{H}}$, unless mentioning explicitly the contrary.

4.3. The Lie algebras $\widehat{\mathfrak{g l}}_{m}$ and $\widetilde{\mathfrak{g l}}_{m}$. We define the Lie algebra $\widehat{\mathfrak{g l}}_{m}$ in the same way as $\widehat{\mathfrak{s l}}_{m}$, with $\mathfrak{g l}_{m}$ instead of $\mathfrak{s l}_{m}$. We'll also use the extended affine Lie algebra $\widetilde{\mathfrak{g l}}_{m}$, obtained by adding to $\widehat{\mathfrak{g l}}_{m}$ the 1 -dimensional vector space spanned by the scaling element $D$ such that $\left[D, x \otimes \varpi^{r}\right]=r x \otimes \varpi^{r}$ and $[D, \mathbf{1}]=0$. The Lie algebra

$$
\left(\widehat{\mathfrak{s l}}_{m} \times \mathfrak{H}\right) /(m(\mathbf{1}, 0)-(0, \mathbf{1}))
$$

is isomorphic to $\widehat{\mathfrak{g l}}_{m}$ via the obvious map, which takes the element $b_{r}^{\prime}$ to $\sum_{p=1}^{m} e_{p p} \otimes$ $\varpi^{r}$ and the element $b_{r}$ to $\sum_{p=1}^{m} e_{p p} \otimes \varpi^{-r}$ for each $r>0$. Unless specified otherwise, by a $\widehat{\mathfrak{g l}}_{m}$-module we'll always mean a module over the Lie algebra (4.3), i.e., an $\widehat{\mathfrak{s l}}_{m}$-module with a compatible $\mathfrak{H}$-action. Similarly, by a $\widetilde{\mathfrak{g l}}_{m}$-module we'll always mean a $\widehat{\mathfrak{g l}}_{m}$-module with a scaling operator $D$ such that

$$
\left[D, x \otimes \varpi^{r}\right]=r x \otimes \varpi^{r}, \quad\left[D, b_{r}\right]=-r b_{r}, \quad\left[D, b_{r}^{\prime}\right]=r b_{r}^{\prime} .
$$

Let $P^{\widehat{\mathfrak{s l}}_{m}}$ and $P^{\widetilde{\mathfrak{s l}}_{m}}$ be the weight lattices of $\widehat{\mathfrak{s l}}_{m}$ and $\widetilde{\mathfrak{s l}}_{m}$ respectively. In other words $P^{\widehat{\mathfrak{s l}}_{m}}$ is the lattice spanned by the affine fundamental weights and $P^{\widetilde{\mathfrak{s}}_{m}}$ is the direct sum of $P^{\widehat{\mathfrak{s l}} l_{m}}$ and $\mathbb{Z} \delta$. We may write $P^{\widehat{\mathfrak{g l}}_{m}}=P^{\widehat{\mathfrak{s l}}_{m}}$ and $P^{\widetilde{\mathfrak{g l}}_{m}}=P^{\widetilde{\mathfrak{s l}}_{m}}$. By a dominant integral weight of $\widehat{\mathfrak{g l}}_{m}, \widetilde{\mathfrak{g l}}_{m}$ we'll always mean a dominant integral weight of $\widehat{\mathfrak{s l}}_{m}, \widetilde{\mathfrak{s l}}_{m}$. We denote the sets of such weights by $P_{+}^{\widehat{\mathfrak{g l}}_{m}}, P_{+}^{\widetilde{\mathfrak{g l}}_{m}}$ or by $P_{+}^{\widehat{\mathfrak{s l}}_{m}}$,

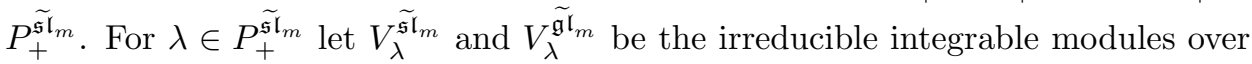
$\widetilde{\mathfrak{s l}}_{m}, \widetilde{\mathfrak{g l}}_{m}$ with the highest weight $\lambda$. We have an isomorphism of $\widetilde{\mathfrak{g l}}_{m}$-modules

$$
V_{\omega_{0}}^{\tilde{\mathfrak{g l}}_{m}}=V_{\omega_{0}}^{\widetilde{\mathfrak{s l}}_{m}} \otimes V_{m}^{\mathfrak{H}} .
$$

Let $Q^{\mathfrak{s l}_{m}}, P^{\mathfrak{s l}_{m}}$ be the root lattice and weight lattice of $\mathfrak{s l}_{m}$. The weights of the module $V_{\omega_{0}}^{\tilde{\mathfrak{s}}}{ }_{m}$ are all the weights of the form

$$
\mu=\omega_{0}+\beta-\frac{1}{2}\langle\beta, \beta\rangle \delta-i \delta, \quad \beta \in Q^{\mathfrak{s l}_{m}}, \quad i \geqslant 0 .
$$

Among those, the extremal weights are the weights for which $i=0$. The set of the extremal weights coincides with the set of the maximal weights, i.e., with the set of the weights $\mu$ such that $\mu+\delta$ is not a weight of $V_{\omega_{0}}^{\tilde{\mathfrak{s}} l_{m}}$. A weight $\mu$ of $V_{\omega_{0}}^{\tilde{\mathfrak{s}} l_{m}}$ is extremal if and only if

$$
\langle\mu, \mu\rangle=0
$$


Note also that we have $\langle\mu, \mu\rangle=-2 i$ if and only if $\mu+i \delta$ is an extremal weight. See, e.g., [4, sec. 20.3, 20.5] for details. For any $\tilde{\mathfrak{s l}}_{m}$-module (resp. $\tilde{\mathfrak{g l}}_{m}$-module) $V$ and any weight $\mu$ we'll denote by $V[\mu]$ the weight subspace in $V$ of weight $\mu$.

Now, let $T_{m}$ be the standard maximal torus in $\mathrm{SL}_{m}$, and let $\mathfrak{t}_{m}$ be its Lie algebra. Let $\widehat{\mathfrak{S}}_{m}$ be the affine symmetric group. It is the semidirect product $\mathfrak{S}_{m} \ltimes Q^{\mathfrak{s l}}$. Note that $Q^{\mathfrak{s l}_{m}}$ is the group of cocharacters of $T_{m}$. We'll regard it as a lattice in $\mathfrak{t}_{m}$ in the usual way, and we'll identify $\mathfrak{t}_{m}$ with $\mathfrak{t}_{m}^{*}$ via the standard invariant pairing on $\mathfrak{t}_{m}$. The $\widehat{\mathfrak{S}}_{m}$-action on $\mathfrak{t}_{m}^{*} \oplus \mathbb{C} \omega_{0} \oplus \mathbb{C} \delta$, see, e.g., [23, sec. 13.1], is such that the element $\beta$ in $Q^{\mathfrak{s l}_{m}}$ acts via the operator

$$
\xi_{\beta}: \mu \mapsto \mu+\mu(\mathbf{1}) \beta-\left(\langle\mu, \beta\rangle+\frac{1}{2}\langle\beta, \beta\rangle \mu(\mathbf{1})\right) \delta .
$$

In particular, we have

$$
\xi_{\beta}\left(\omega_{0}\right)=\omega_{0}+\beta-\frac{1}{2}\langle\beta, \beta\rangle \delta .
$$

We'll use the same notation for the $\widehat{\mathfrak{S}}_{m}$-action on $\mathfrak{t}_{m}^{*} \oplus \mathbb{C} \omega_{0} \oplus \mathbb{C} \delta$ and on $\mathfrak{t}_{m}^{*} \oplus \mathbb{C} \omega_{0}$, hoping it will not create any confusion. Therefore, for $\mu \in \mathfrak{t}_{m}^{*} \oplus \mathbb{C} \omega_{0}$ the symbol $\xi_{\beta}(\mu)$ will denote both the weight given by (4.4) and the weight $\mu+\mu(\mathbf{1}) \beta$. We can view the cocharacter $\beta \in Q^{\mathfrak{s l}_{m}}$ as a group-scheme homomorphism $\mathbb{G}_{m} \rightarrow T_{m}$. Thus the image $\beta(\varpi)$ of the element $\varpi \in K$ lies in $T_{m}(K)$. The coadjoint action of $\beta(\varpi)$ on $\mathfrak{t}_{m}^{*} \oplus \mathbb{C} \omega_{0} \oplus \mathbb{C} \delta$ is given by $\xi_{\beta}^{-1}$; see, e.g., [30. Therefore for any integrable $\widetilde{\mathfrak{s l}}_{m}$-module $V$ we have

$$
\beta(V[\mu])=V\left[\xi_{\beta}^{-1}(\mu)\right] .
$$

4.4. The Hopf $\mathbb{C}$-algebra $\lambda_{\Gamma}$. Now, let us consider the Hopf $\mathbb{C}$-algebras $R(\Gamma)$. Once again, the multiplication/comultiplication on $R(\Gamma)$ is given by the induction/restriction. We equip $R(\Gamma)$ with the symmetric $\mathbb{C}$-bilinear form given by

$$
\langle f, g\rangle=\left|\Gamma_{n}\right|^{-1} \sum_{x \in \Gamma_{n}} f(x) g\left(x^{-1}\right), \quad f, g \in\left[\operatorname{Rep}\left(\mathbb{C} \Gamma_{n}\right)\right] .
$$

Here we regard $f, g$ as characters of $\mathbb{C} \Gamma_{n}$. This bilinear form is a Hopf pairing. Next, we consider the Hopf $\mathbb{C}$-algebra $\boldsymbol{\Lambda}_{\Gamma}=\boldsymbol{\Lambda}^{\otimes \Gamma}$. We'll use the following elements in $\boldsymbol{\Lambda}_{\Gamma}$ :

$$
f^{\gamma}=1 \otimes \cdots \otimes 1 \otimes f \otimes 1 \otimes \cdots \otimes 1, \quad f \in \Lambda, \quad \gamma \in \Gamma,
$$

with $f$ at the $\gamma$-th place. We abbreviate

$$
P_{\mu}^{\gamma}=\left(P_{\mu}\right)^{\gamma}, \quad P_{\lambda}=\prod_{\gamma \in \Gamma} P_{\lambda(\gamma)}^{\gamma}, \quad \mu \in \mathcal{P}, \quad \lambda \in \mathcal{P}^{\Gamma} .
$$

The comultiplication in $\boldsymbol{\Lambda}_{\Gamma}$ is characterized by

$$
\Delta\left(P_{r}^{\gamma}\right)=P_{r}^{\gamma} \otimes 1+1 \otimes P_{r}^{\gamma}, \quad r>0, \quad \gamma \in \Gamma .
$$

Following [27, chap. I, app. B, (7.1)] we write

$$
P_{r, p}=\ell^{-1} \sum_{\gamma \in \Gamma} \gamma^{p} P_{r}^{\gamma}, \quad r \geqslant 0, \quad p \in \mathbb{Z}_{\ell}
$$

We equip $\boldsymbol{\Lambda}_{\Gamma}$ with the Hopf pairing such that

$$
\left\langle P_{r, p}, P_{s, q}\right\rangle=r \delta_{p, q} \delta_{r, s}, \quad r, s>0, \quad p, q \in \mathbb{Z}_{\ell} .
$$

The elements $P_{r, p}, r>0$, are algebraically independent and generate the $\mathbb{C}$-algebra $\boldsymbol{\Lambda}_{\Gamma}$. Thus, we may regard $P_{r, p}, r>0$, as the $r$-th power sum of a new sequence of 
variables $x_{i, p}, i>0$. We do not need to construct explicitly the elements $x_{i, p}, i>0$. They are auxiliary variables which are only used to define the following elements:

$$
S_{\mu, p}=S_{\mu}\left(x_{i, p}\right), \quad S_{\lambda}=\prod_{p \in \mathbb{Z}_{\ell}} S_{\lambda(p), p}, \quad \mu \in \mathcal{P}, \quad \lambda \in \mathcal{P}^{\ell} .
$$

Here, the symbol $S_{\mu}$ denotes the Schur function associated to the partition $\mu$. By construction, the elements $S_{\lambda}$ are indeed polynomials in the $P_{r, p}$ 's. So they belong to $\boldsymbol{\Lambda}_{\Gamma}$. The Hopf $\mathbb{C}$-algebras $R(\Gamma)$ and $\boldsymbol{\Lambda}_{\Gamma}$ are identified via the characteristic map [27, chap. I, app. B, (6.2)]

$$
\operatorname{ch}: R(\Gamma) \rightarrow \Lambda_{\Gamma}
$$

This map intertwines the induction in $R(\Gamma)$ with the multiplication in $\boldsymbol{\Lambda}_{\Gamma}$ by 27 , chap. I, app. B, (6.3)]. By [27, chap. I, app. B, (9.4)] and (3.4) we have

$$
\operatorname{ch}\left(\bar{L}_{\lambda}\right)=S_{\tau \lambda}, \quad \lambda \in \mathcal{P}^{\ell},
$$

where $\tau$ is the permutation of $\mathcal{P}^{\ell}$ such that $(\tau \lambda)(p)=\lambda(p+1)$ for each $p \in \mathbb{Z}_{\ell}$. For $\lambda \in \mathcal{P}^{\Gamma}$ we write

$$
z_{\lambda}=\prod_{\gamma \in \Gamma} z_{\lambda(\gamma)} \ell^{l(\lambda(\gamma))}
$$

where $z_{\lambda(\gamma)}$ is as in (3.1), and we define $\bar{\lambda} \in \mathcal{P}^{\Gamma}$ by $\bar{\lambda}(\gamma)=\lambda\left(\gamma^{-1}\right)$. Then we have

$$
\begin{array}{r}
\left\langle S_{\lambda}, S_{\mu}\right\rangle=\delta_{\lambda, \mu}, \quad \lambda, \mu \in \mathcal{P}^{\ell}, \\
\left\langle P_{\lambda}, P_{\bar{\mu}}\right\rangle=\delta_{\lambda, \mu} z_{\lambda}, \quad \lambda, \mu \in \mathcal{P}^{\Gamma} .
\end{array}
$$

The first equality is proved as in [27, chap. I, app. B, (7.4)], while the second one is [27, chap. I, app. B, $\left.\left(5.3^{\prime}\right)\right]$. By (4.7), (4.8) the map ch is an isometry. Thus it intertwines the restriction in $R(\Gamma)$ with the comultiplication in $\boldsymbol{\Lambda}_{\Gamma}$.

Proposition 4.1. (a) The restriction $\operatorname{Rep}\left(\mathbb{C} \Gamma_{n}\right) \rightarrow \operatorname{Rep}\left(\mathbb{C S}_{n}\right)$ yields the $\mathbb{C}$-algebra homomorphism $\operatorname{Res}_{\mathfrak{S}}^{\Gamma}: \boldsymbol{\Lambda}_{\Gamma} \rightarrow \boldsymbol{\Lambda}$ such that $S_{\lambda} \mapsto \prod_{p} S_{\lambda(p)}, P_{r, p} \mapsto P_{r}$.

(b) The induction $\operatorname{Rep}\left(\mathbb{C S}_{n}\right) \rightarrow \operatorname{Rep}\left(\mathbb{C}_{n}\right)$ yields the $\mathbb{C}$-algebra homomorphism $\operatorname{Ind}_{\mathfrak{S}}^{\Gamma}: \boldsymbol{\Lambda} \rightarrow \boldsymbol{\Lambda}_{\Gamma}$ such that $P_{r} \mapsto P_{r}^{1}=\sum_{p \in \mathbb{Z}_{\ell}} P_{r, p}$.

Proof. First, we concentrate on $(a)$. Recall that $\operatorname{Rep}\left(\mathbb{C} \Gamma_{n}\right), \operatorname{Rep}\left(\mathbb{C S}_{n}\right)$ are identified with $\boldsymbol{\Lambda}_{\Gamma}, \boldsymbol{\Lambda}$ via the characteristic map, which is a $\mathbb{C}$-algebra isomorphism. Under this map, the restriction $\operatorname{Rep}\left(\mathbb{C} \Gamma_{n}\right) \rightarrow \operatorname{Rep}\left(\mathbb{C S}_{n}\right)$, which is a $\mathbb{C}$-algebra homomorphism, can be regarded as a $\mathbb{C}$-algebra homomorphism $\operatorname{Res}_{\mathfrak{S}}^{\Gamma}: \boldsymbol{\Lambda}_{\Gamma} \rightarrow \boldsymbol{\Lambda}$. It takes $S_{\lambda}$ to $\prod_{p} S_{\lambda(p)}$ by formula (4.7), because, by formula (3.4), the simple module $\bar{L}_{\tau^{-1} \lambda}$ is induced from the tensor product of the representations associated with the $S_{\lambda(p)}$ 's. To prove that $\operatorname{Res}_{\mathfrak{S}}^{\Gamma_{\mathfrak{S}}}\left(P_{r, p}\right)=P_{r}$, observe that

$$
\operatorname{ch}\left(\sigma_{r, p}\right)=P_{r, p}, \quad r>0,
$$

where $\sigma_{r, p}$ is the class function on $\Gamma_{r}$ which takes the value $r\left(\gamma_{1} \gamma_{2} \cdots \gamma_{r}\right)^{p}$ on pairs $\left(w,\left(\gamma_{1}, \gamma_{2}, \ldots, \gamma_{r}\right)\right)$ such that $w$ is an $r$-cycle, and 0 elsewhere; see [12, lem. 5.1]. Now, it is easy to see that the restriction of the class function $\sigma_{r, p}$ to the group $\mathfrak{S}_{n}$ is precisely the class function associated to the symmetric polynomial $P_{r}$. Now we concentrate on $(b)$. Note that

$$
\operatorname{Res}_{\mathfrak{S}}^{\Gamma}\left(P_{0}^{\gamma}\right)=1, \quad \operatorname{Res}_{\mathfrak{S}}^{\Gamma}\left(P_{r}^{\gamma}\right)=\ell \delta_{\gamma, 1} P_{r}, \quad r>0 .
$$


Therefore, for $\lambda \in \mathcal{P}^{\Gamma}$ we have

$$
\operatorname{Res}_{\mathfrak{S}}^{\Gamma}\left(P_{\lambda}\right)=\prod_{\gamma \in \Gamma} \operatorname{Res}_{\mathfrak{S}}^{\Gamma}\left(P_{\lambda(\gamma)}^{\gamma}\right)= \begin{cases}\ell^{l(\lambda(1))} P_{\lambda(1)} & \text { if } \lambda(\gamma)=\emptyset \text { for } \gamma \neq 1 \\ 0 & \text { else. }\end{cases}
$$

If $f, g \in\left[\operatorname{Rep}\left(\mathbb{C} \Gamma_{n}\right)\right]$ are the characters of finite-dimensional $\Gamma_{n}$-modules $V, W$, then $\langle f, g\rangle$ is the dimension of the space of $\mathbb{C} \Gamma_{n}$-linear maps $V \rightarrow W$. Hence, by Frobenius reciprocity, the operator $\operatorname{Ind}_{\mathfrak{S}}^{\Gamma}$ is adjoint to the operator $\operatorname{Res}_{\mathfrak{S}}^{\Gamma}$. Thus,

$$
\left\langle\operatorname{Ind}_{\mathfrak{S}}^{\Gamma}\left(P_{r}\right), P_{\lambda}\right\rangle= \begin{cases}r \ell^{l(\lambda(1))} \delta_{\lambda(1),(r)} & \text { if } \lambda(\gamma)=\emptyset \text { for } \gamma \neq 1, \\ 0 & \text { else. }\end{cases}
$$

This implies that $\operatorname{Ind}_{\mathfrak{S}}^{\Gamma}\left(P_{r}\right)=a P_{r}^{1}$ for some $a$. To determine $a$, let $\lambda$ be such that $\lambda(\gamma)=\emptyset$ if $\gamma \neq 1$ and $\lambda(1)=(r)$. Then we have

$$
P_{\lambda}=P_{r}^{1}, \quad\left\langle P_{\lambda}, P_{\lambda}\right\rangle=r \ell .
$$

This implies that $a=1$.

Remark 4.2. Let $f \mapsto \bar{f}$ be the $\mathbb{C}$-antilinear involution of $\boldsymbol{\Lambda}_{\Gamma}$ which fixes the $P_{\lambda}$ 's with $\lambda \in \mathcal{P}^{\Gamma}$; see [27, chap. I, app. B, (5.2)]. For $\lambda \in \mathcal{P}^{\ell}$ let $\bar{\lambda}$ be the $\ell$-partition given by $\bar{\lambda}(p)=\lambda(-p)$. We have

$$
\bar{P}_{r, p}=P_{r,-p}, \quad \bar{S}_{\lambda}=S_{\bar{\lambda}}, \quad r>0, \quad p \in \mathbb{Z}_{\ell}, \quad \lambda \in \mathcal{P}^{\ell} .
$$

Remark 4.3. Setting $\ell=1$ in $\boldsymbol{\Lambda}_{\Gamma}$ we get the standard Hopf algebra structure and the Hopf pairing of $\boldsymbol{\Lambda}$.

Remark 4.4. We have [27, chap. I, app. B, $\left(7.1^{\prime}\right)$ ]

$$
P_{r}^{\gamma}=\sum_{p \in \mathbb{Z}_{\ell}} \gamma^{-p} P_{r, p}, \quad r \geqslant 0, \quad P_{0}^{\gamma}=1, \quad P_{0, p}=\delta_{0, p} .
$$

4.5. The level 1 Fock space. Fix once and for all a basis $\left(\epsilon_{1}, \ldots, \epsilon_{m}\right)$ of $\mathbb{C}^{m}$. The level 1 Fock space of $\widehat{\mathfrak{s l}}_{m}$ is the space $\mathcal{F}_{m}$ of semi-infinite wedges of the $\mathbb{C}$-vector space $V_{m}=\mathbb{C}^{m} \otimes \mathbb{C}\left[t, t^{-1}\right]$. More precisely, we have

$$
\mathcal{F}_{m}=\bigoplus_{d \in \mathbb{Z}} \mathcal{F}_{m}^{(d)}
$$

where $\mathcal{F}_{m}^{(d)}$ is the subspace spanned by the semi-infinite wedges of charge $d$, i.e., the semi-infinite wedges of the form

$$
u_{i_{1}} \wedge u_{i_{2}} \wedge \cdots, \quad i_{1}>i_{2}>\ldots, \quad u_{i-j m}=\epsilon_{i} \otimes t^{j},
$$

where $i_{k}=d-k+1$ if $k \gg 0$. We write

$$
|\lambda, d\rangle=u_{i_{1}} \wedge u_{i_{2}} \wedge \cdots, \quad \lambda \in \mathcal{P}, \quad i_{k}=\lambda_{k}+d-k+1, \quad k>0 .
$$

The elements $|\lambda, d\rangle$ with $\lambda \in \mathcal{P}$ form a basis of $\mathcal{F}_{m}^{(d)}$. We equip $\mathcal{F}_{m}^{(d)}$ with the $\mathbb{C}$-bilinear symmetric form such that this basis is orthonormal.

The Fock space $\mathcal{F}_{m}^{(d)}$ is equipped with a level one representation of $\widehat{\mathfrak{s l}}_{m}$ in the following way. First, the $\mathbb{C}$-vector space $V_{m}$ is given the level 0 action of $\widehat{\mathfrak{s l}}_{m}$ induced by the homomorphism

$$
\widehat{\mathfrak{s l}}_{m} \rightarrow \mathfrak{s l}_{m} \otimes \mathbb{C}\left[t, t^{-1}\right], \quad \mathbf{1} \mapsto 0, \quad x \otimes \varpi \mapsto x \otimes t
$$


and the obvious actions of $\mathfrak{s l}_{m}$ and $\mathbb{C}\left[t, t^{-1}\right]$ on $V_{m}$. Then, taking semi-infinite wedges, this action yields a level 1 action of $\widehat{\mathfrak{s l}}_{m}$ on $\mathcal{F}_{m}^{(d)}$; see, e.g., 35.

Next, observe that the multiplication by $t^{r}, r>0$, yields an endomorphism of $V_{m}$. Taking semi-infinite wedges it yields a linear operator $b_{r}$ on $\mathcal{F}_{m}^{(d)}$. Let $b_{r}^{\prime}$ be the adjoint of $b_{r}$. Then $b_{r}^{\prime}, b_{r}$ define a level $m$ action of $\mathfrak{H}$ on $\mathcal{F}_{m}^{(d)}$. The $\widehat{\mathfrak{s l}}_{m}$-action and the $\mathfrak{H}$-action on $\mathcal{F}_{m}^{(d)}$ glue together, yielding a level 1 representation of $\widehat{\mathfrak{g l}}_{m}$ on $\mathcal{F}_{m}^{(d)}$; see 35 again.

We have an isomorphism of (simple) $\widehat{\mathfrak{g l}}_{m}$-modules

$$
\mathcal{F}_{m}^{(d)}=V_{\omega_{d \bmod m}}^{\widehat{\mathfrak{g l}}_{m}} ;
$$

see [19, chap. 14] and the references there. It is unique up to a scalar. It identifies the symmetric bilinear form of $\mathcal{F}_{m}^{(d)}$ with the Shapovalov form on $V_{\omega_{d \bmod m}}^{\widehat{\mathrm{gl}_{m}}}$, i.e., with the unique (up to a scalar) symmetric bilinear form such that the adjoints of $b_{r}, e_{q}$ are $b_{r}^{\prime}, f_{q}$ respectively.

Remark 4.5. The $\mathbb{C}$-linear isomorphism

$$
\mathcal{F}_{m}^{(d)} \rightarrow \Lambda, \quad|\lambda, d\rangle \mapsto S_{\lambda}, \quad \lambda \in \mathcal{P}
$$

takes the operators $b_{r}^{\prime}, b_{r}, e_{q}, f_{q}$ on the left-hand side to the operators $b_{m r}^{\prime}, b_{m r}$, $e_{q-d}, f_{q-d}$ on the right-hand side.

4.6. The level $\ell$ Fock space. Fix a basis $\left(\epsilon_{1}, \ldots, \epsilon_{m}\right)$ of $\mathbb{C}^{m}$ and a basis $\left(\dot{\epsilon}_{1}, \ldots, \dot{\epsilon}_{\ell}\right)$ of $\mathbb{C}^{\ell}$. The level $\ell$ Fock space of $\widehat{\mathfrak{s l}}_{m}$ is the $\mathbb{C}$-vector space

$$
\mathcal{F}_{m, \ell}=\bigoplus_{d \in \mathbb{Z}} \mathcal{F}_{m, \ell}^{(d)}
$$

of semi-infinite wedges of the $\mathbb{C}$-vector space $V_{m, \ell}=\mathbb{C}^{m} \otimes \mathbb{C}^{\ell} \otimes \mathbb{C}\left[z, z^{-1}\right]$. The latter are defined as in (4.9) with

$$
u_{i+(j-1) m-k m \ell}=\epsilon_{i} \otimes \dot{\epsilon}_{j} \otimes z^{k} .
$$

Here $i=1, \ldots, m, j=1, \ldots, \ell$, and $k \in \mathbb{Z}$. Using the semi-infinite wedges of these elements, we define basis elements $|\lambda, d\rangle$ of $\mathcal{F}_{m, \ell}^{(d)}$ as in (4.10) with $\lambda$ running over $\mathcal{P}$. We equip $\mathcal{F}_{m, \ell}^{(d)}$ with the $\mathbb{C}$-bilinear symmetric form such that the basis elements $|\lambda, d\rangle$ are orthonormal. This yields a $\mathbb{C}$-linear isomorphism

$$
\mathcal{F}_{m, \ell}^{(d)} \rightarrow \Lambda, \quad|\lambda, d\rangle \mapsto S_{\lambda}, \quad \lambda \in \mathcal{P} .
$$

We equip the $\mathbb{C}$-vector space $\mathcal{F}_{m, \ell}^{(d)}$ with the following actions; see 35] for details:

- The level $m \ell$ action of $\mathfrak{H}$ such that $b_{r}^{\prime}, b_{r}$ are taken to the operators $b_{m \ell r}^{\prime}$, $b_{m \ell r}$ on $\boldsymbol{\Lambda}$ under the isomorphism (4.15) for $r>0$.

- The level $\ell$ action of $\widehat{\mathfrak{s l}}_{m}$ defined as follows: equip the $\mathbb{C}\left[z, z^{-1}\right]$-module $V_{m, \ell}$ with the level 0 action of $\widehat{\mathfrak{s l}}_{m}$ given by the evaluation homomorphism (4.11) and the obvious actions of $\mathfrak{s l}_{m}$ and $\mathbb{C}\left[z, z^{-1}\right]$ on $V_{m, \ell}$. Taking semi-infinite wedges we get a level $\ell$ action of $\widehat{\mathfrak{s l}}_{m}$ on $\mathcal{F}_{m, \ell}^{(d)}$.

- The level $m$ action of $\widehat{\mathfrak{s l}}_{\ell}$ which is defined as above by exchanging the roles of $m$ and $\ell$. 
The actions of $\mathfrak{H}, \widehat{\mathfrak{s l}}_{m}$ and $\widehat{\mathfrak{s l}}_{\ell}$ commute with each other. An $\ell$-tuple of integers $s=\left(s_{p}\right)$ is called an $\ell$-charge of weight $d$ if $\sum_{p} s_{p}=d$. Set

$$
\hat{\gamma}(s, m)=\left(m-s_{1}+s_{\ell}\right) \omega_{0}+\sum_{p=1}^{\ell-1}\left(s_{p}-s_{p+1}\right) \omega_{p} .
$$

The Fock space associated with the $\ell$-charge $s$ is the subspace

$$
\mathcal{F}_{m, \ell}^{(s)}=\mathcal{F}_{m, \ell}^{(d)}[\hat{\gamma}(s, m)]
$$

consisting of the elements of weight $\hat{\gamma}(s, m)$ with respect to the $\widehat{\mathfrak{s l}}_{\ell}$-action. It is an $\widehat{\mathfrak{s l}}_{m} \times \mathfrak{H}$-submodule of $\mathcal{F}_{m, \ell}^{(d)}$. Consider the basis elements $|\lambda, s\rangle, \lambda \in \mathcal{P}^{\ell}$, of $\mathcal{F}_{m, \ell}^{(s)}$ defined in [35, sec. 4.1]. The representation of $\widehat{\mathfrak{s l}}_{m}$ on $\mathcal{F}_{m, \ell}^{(s)}$ can be characterized in the following way, see e.g., [18, 35],

$$
e_{q}|\lambda, s\rangle=\sum_{\nu}|\nu, s\rangle, \quad f_{q}|\lambda, s\rangle=\sum_{\mu}|\mu, s\rangle
$$

where $\nu$ (resp. $\mu$ ) runs through all $\ell$-partitions obtained by removing (resp. adding) a node of coordinate $(i, j)$ in the $p$-th partition of $\lambda$ such that $q=s_{p}+j-i$ modulo $m$. Consider the $\mathbb{C}$-vector space isomorphism

$$
\Lambda_{\Gamma} \rightarrow \mathcal{F}_{m, \ell}^{(s)}, \quad S_{\tau \lambda} \mapsto|\lambda, s\rangle, \quad \lambda \in \mathcal{P}^{\ell}
$$

By [35, sec. 4.1] we have an equality of sets

$$
\left\{|\lambda, s\rangle: \lambda \in \mathcal{P}^{\ell}, s=\left(s_{p}\right) \in \mathbb{Z}^{\ell}, \sum_{p} s_{p}=d\right\}=\{|\lambda, d\rangle: \lambda \in \mathcal{P}\} .
$$

Thus the elements $|\lambda, s\rangle$ form an orthonormal basis of $\mathcal{F}_{m, \ell}^{(d)}$ and the map (4.19) preserves the pairings by (4.8). The representation of $\mathfrak{H}$ on $\mathcal{F}_{m, \ell}^{(s)}$ can be characterized in the following way.

Proposition 4.6. The operators $b_{r}^{\prime}, b_{r}, r>0$, on $\mathcal{F}_{m, \ell}^{(s)}$ are adjoint to each other. Further $b_{r}$ acts as the multiplication by the element $P_{m r}^{1}=\sum_{p} P_{m r, p}$ of $\boldsymbol{\Lambda}_{\Gamma}$ under the isomorphism (4.19).

Proof. The first claim is [35, prop. 5.8]. To prove the second claim, consider the isomorphism of vector spaces

$$
\mathcal{F}_{m, \ell}^{(s)} \stackrel{\sim}{\rightarrow} \bigotimes_{p \in \mathbb{Z}_{\ell}} \mathcal{F}_{m}^{\left(s_{p}\right)}, \quad|\lambda, s\rangle \mapsto \bigotimes_{p \in \mathbb{Z}_{\ell}}\left|\tau \lambda(p), s_{p}\right\rangle .
$$

Recall that $\tau \lambda(p)=\lambda(p+1)$. Next, for each $p \in \mathbb{Z}_{\ell}$ let $\Lambda^{(p)}$ be the subalgebra of $\Lambda_{\Gamma}$ generated by $P_{r, p}, r>0$. We have a canonical algebra isomorphism

$$
\Lambda^{(p)} \stackrel{\sim}{\rightarrow} \Lambda, \quad P_{r, p} \mapsto P_{r}, S_{\lambda, p} \mapsto S_{\lambda}, \forall \lambda \in \mathcal{P} ;
$$

see Section 4.4, Further, we have

$$
\bigotimes_{p \in \mathbb{Z}_{\ell}} \Lambda^{(p)} \stackrel{\sim}{\rightarrow} \Lambda_{\Gamma}, \quad \bigotimes_{p \in \mathbb{Z}_{\ell}} x_{p} \mapsto \prod_{p \in \mathbb{Z}_{\ell}} x_{p}
$$


These isomorphisms fit into a commutative diagram

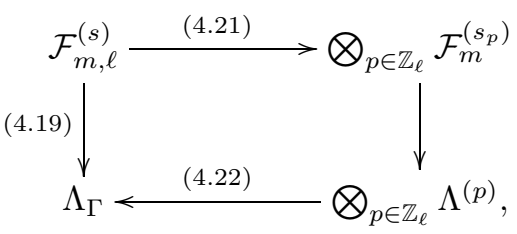

where the right vertical arrow is given by (4.13) on each component. Now, the formulas in [35, sec. 4.1, 4.3 and (25)] imply that (4.21) intertwines the operator $b_{r}$ on $\mathcal{F}_{m, \ell}^{(s)}$ and the operator

$$
b_{r} \otimes 1 \otimes \cdots \otimes 1+1 \otimes b_{r} \otimes 1 \otimes \cdots \otimes 1+\cdots+1 \otimes \cdots \otimes 1 \otimes b_{r}
$$

on $\bigotimes_{p \in \mathbb{Z}_{\ell}} \mathcal{F}_{m}^{\left(s_{p}\right)}$. By Remark 4.5 the map (4.13) sends the operator $b_{r}$ on $\mathcal{F}_{m}^{\left(s_{p}\right)}$ to the operator $b_{m r}$ on $\Lambda^{(p)}$. The latter is given by the multiplication by $P_{m r, p}$. Therefore $b_{r}$ acts on $\mathcal{F}_{m, \ell}^{(s)}$ by the multiplication by $\sum_{p \in \mathbb{Z}_{\ell}} P_{m r, p}=P_{m r}^{1}$.

Remark 4.7. The $\widehat{\mathfrak{s l}}_{m}$-action on $\mathcal{F}_{m, \ell}^{(s)}$ can be extended to an $\widetilde{\mathfrak{s l}}_{m}$-action such that the weight of $|\lambda, s\rangle$ is

$$
-\Delta(s, m) \delta+\sum_{p=1}^{\ell} \omega_{s_{p} \bmod m}-\sum_{q=0}^{m-1} n_{q}(\lambda) \alpha_{q}
$$

see [35, sec. 4.2]. Here $n_{q}(\lambda)$ is the number of $q$-nodes in $\lambda$; i.e., it is the sum over all $p$ 's of the number of nodes of coordinate $(i, j)$ in the $p$-th partition of $\lambda$ such that $s_{p}+j-i=q \bmod m$. The integer $s_{p}+j-i$ is called the content of the node $(i, j)$ of $\lambda$. We also have used the notation

$$
\Delta(s, m)=\frac{1}{2} \sum_{p=1}^{\ell}\left\langle\omega_{s_{p} \bmod m}, \omega_{s_{p} \bmod m}\right\rangle+\frac{1}{2} \sum_{p=1}^{\ell} s_{p}\left(s_{p} / m-1\right) .
$$

In particular, we have

$$
D(|\lambda, s\rangle)=-\left(\Delta(s, m)+n_{0}(\lambda)\right)|\lambda, s\rangle .
$$

\section{The CAtegorification of the Heisenberg ALGebra}

We'll abbreviate

$$
\mathcal{O}(\Gamma)=\bigoplus_{n \geqslant 0} \mathcal{O}\left(\Gamma_{n}\right)
$$

Assume that $h, h_{p}$ are rational numbers as in (3.9). Thus $\Lambda$ is a rational weight of $\widehat{\mathfrak{s l}} \ell_{\ell}$ of level 1. Let $m$ be the denominator of $h$. We'll assume that $m>2$.

5.1. The functors $A_{\lambda, !}, A_{\lambda}^{*}, A_{\lambda, *}$ on $D^{b}(\mathcal{O}(\Gamma))$. To simplify the exposition, from now on we'll assume that $\ell>1$. All the statements below have an analogous version for $\ell=1$, by replacing everywhere $\mathbb{C}^{n}$ by $\mathbb{C}_{0}^{n}$. Let $n, r$ be nonnegative integers. Consider the point

$$
b_{n, r}=(0, \ldots, 0,1, \ldots, 1) \in \mathfrak{h}=\mathbb{C}^{n+r},
$$

with $x_{i}=0$ for $1 \leqslant i \leqslant n$, and $x_{i}=1$ for $n<i \leqslant n+r$. The centralizer of $b_{n, r}$ in $\Gamma_{n+r}$ is the parabolic subgroup $\Gamma_{n, r}$. We have

$$
\mathfrak{h} / \mathfrak{h}^{\Gamma_{n, r}}=\mathbb{C}^{n} \times \mathbb{C}_{0}^{r} .
$$


Here $\mathbb{C}^{n}$ is the reflection representation of $\Gamma_{n}$ and $\mathbb{C}_{0}^{r}$ is the reflection representation of $\mathfrak{S}_{r}$. Note that

$$
\mathcal{O}\left(\Gamma_{n, r}\right)=\mathcal{O}\left(\Gamma_{n, r}, \mathbb{C}^{n} \times \mathbb{C}_{0}^{r}\right), \quad \mathcal{O}\left(\mathfrak{S}_{r}\right)=\mathcal{O}\left(\mathfrak{S}_{r}, \mathbb{C}_{0}^{r}\right) .
$$

In particular we have a canonical equivalence of categories

$$
\mathcal{O}\left(\Gamma_{n, r}\right)=\mathcal{O}\left(\Gamma_{n}\right) \otimes \mathcal{O}\left(\mathfrak{S}_{r}\right)
$$

Thus the induction and restriction relative to $b_{n, r}$ yield functors

$$
\begin{aligned}
& \mathcal{O}_{\operatorname{Ind}_{n, r}}: \mathcal{O}\left(\Gamma_{n}\right) \otimes \mathcal{O}\left(\mathfrak{S}_{r}\right) \rightarrow \mathcal{O}\left(\Gamma_{n+r}\right), \\
& { }^{\mathcal{O}} \operatorname{Res}_{n, r}: \mathcal{O}\left(\Gamma_{n+r}\right) \rightarrow \mathcal{O}\left(\Gamma_{n}\right) \otimes \mathcal{O}\left(\mathfrak{S}_{r}\right) .
\end{aligned}
$$

Now consider the functors ${ }^{\mathcal{O}} \operatorname{Ind}_{n, m r},{ }^{\mathcal{O}} \operatorname{Res}_{n, m r}$. The parameters of $H\left(\Gamma_{n+m r}\right)$ and $H\left(\Gamma_{n}\right)$ are $h, \Lambda$. The parameter of $H\left(\mathfrak{S}_{m r}\right)$ is $h$. Fix a partition $\lambda \in \mathcal{P}_{r}$. We define the functors

$$
\begin{aligned}
& \mathcal{O}\left(\Gamma_{n}\right) \otimes \mathcal{O}\left(\mathfrak{S}_{m r}\right) \rightarrow \mathcal{O}\left(\Gamma_{n}\right), \\
& M \mapsto \operatorname{Hom}_{\mathcal{O}\left(\mathfrak{S}_{m r}\right)}\left(M, L_{m \lambda}\right)^{\circledast}, \quad M \mapsto \operatorname{Hom}_{\mathcal{O}\left(\mathfrak{S}_{m r}\right)}\left(L_{m \lambda}, M\right),
\end{aligned}
$$

as the tensor product of the identity of $\mathcal{O}\left(\Gamma_{n}\right)$ and of the functors

$$
\begin{aligned}
& \mathcal{O}\left(\mathfrak{S}_{m r}\right) \rightarrow \operatorname{Rep}(\mathbb{C}), \\
& M \mapsto \operatorname{Hom}_{\mathcal{O}\left(\mathfrak{S}_{m r}\right)}\left(M, L_{m \lambda}\right)^{*}, \quad M \mapsto \operatorname{Hom}_{\mathcal{O}\left(\mathfrak{S}_{m r}\right)}\left(L_{m \lambda}, M\right) .
\end{aligned}
$$

Here the superscript $*$ denotes the dual $\mathbb{C}$-vector space. Note that the space $\operatorname{Hom}_{\mathcal{O}\left(\mathfrak{S}_{m r}\right)}\left(M, L_{m \lambda}\right)$ is finite dimensional because the category $\mathcal{O}\left(\mathfrak{S}_{m r}\right)$ is Artinian. Thus, given a decomposition $M=\bigoplus_{i} M_{i} \otimes N_{i}$, we get

$$
\begin{aligned}
\operatorname{Hom}_{\mathcal{O}\left(\mathfrak{S}_{m r}\right)}\left(M, L_{m \lambda}\right)^{\circledast} & =\bigoplus_{i} M_{i} \otimes \operatorname{Hom}_{\mathcal{O}\left(\mathfrak{S}_{m r}\right)}\left(N_{i}, L_{m \lambda}\right)^{*}, \\
\operatorname{Hom}_{\mathcal{O}\left(\mathfrak{S}_{m r}\right)}\left(L_{m \lambda}, M\right) & =\bigoplus_{i} M_{i} \otimes \operatorname{Hom}_{\mathcal{O}\left(\mathfrak{S}_{m r}\right)}\left(L_{m \lambda}, N_{i}\right) .
\end{aligned}
$$

The functor $\operatorname{Hom}_{\mathcal{O}\left(\mathfrak{S}_{m r}\right)}\left(\bullet, L_{m \lambda}\right)^{\circledast}$ is right exact and the functor $\operatorname{Hom}_{\mathcal{O}\left(\mathfrak{S}_{m r}\right)}\left(L_{m \lambda}, \bullet\right)$ is left exact. We denote the corresponding derived functors in the following way:

$$
M \mapsto \operatorname{RHom}_{D^{b}\left(\mathcal{O}\left(\mathfrak{S}_{m r}\right)\right)}\left(M, L_{m \lambda}\right)^{\circledast}, \quad M \mapsto \operatorname{RHom}_{D^{b}\left(\mathcal{O}\left(\mathfrak{S}_{m r}\right)\right)}\left(L_{m \lambda}, M\right) .
$$

Definition 5.1. For $\lambda \in \mathcal{P}_{r}$ with $r \geqslant 0$ we define the functors

$$
\begin{array}{ll}
A_{\lambda, !}: D^{b}\left(\mathcal{O}\left(\Gamma_{n+m r}\right)\right) \rightarrow D^{b}\left(\mathcal{O}\left(\Gamma_{n}\right)\right), & M \mapsto \operatorname{RHom}_{D^{b}\left(\mathcal{O}\left(\mathfrak{S}_{m r}\right)\right)}\left({ }^{\mathcal{O}} \operatorname{Res}_{n, m r}(M), L_{m \lambda}\right)^{\circledast}, \\
A_{\lambda}^{*}: D^{b}\left(\mathcal{O}\left(\Gamma_{n}\right)\right) \rightarrow D^{b}\left(\mathcal{O}\left(\Gamma_{n+m r}\right)\right), \quad M \mapsto{ }^{\mathcal{O}} \operatorname{Ind}_{n, m r}\left(M \otimes L_{m \lambda}\right), \\
A_{\lambda, *}: D^{b}\left(\mathcal{O}\left(\Gamma_{n+m r}\right)\right) \rightarrow D^{b}\left(\mathcal{O}\left(\Gamma_{n}\right)\right), \quad M \mapsto \operatorname{RHom}_{D^{b}\left(\mathcal{O}\left(\mathfrak{S}_{m r}\right)\right)}\left(L_{m \lambda},{ }^{\mathcal{O}} \operatorname{Res}_{n, m r}(M)\right) .
\end{array}
$$

Proposition 5.2. We have a triple of exact adjoint endofunctors $\left(A_{\lambda, !}, A_{\lambda}^{*}, A_{\lambda, *}\right)$ of the triangulated category $D^{b}(\mathcal{O}(\Gamma))$. For $M, N \in D^{b}(\mathcal{O}(\Gamma))$ we have

$$
\begin{aligned}
& \operatorname{RHom}_{D^{b}(\mathcal{O}(\Gamma))}\left(A_{\lambda}^{*}(M), N\right)=\operatorname{RHom}_{D^{b}(\mathcal{O}(\Gamma))}\left(M, A_{\lambda, *}(N)\right), \\
& \operatorname{RHom}_{D^{b}(\mathcal{O}(\Gamma))}\left(A_{\lambda, !}(M), N\right)=\operatorname{RHom}_{D^{b}(\mathcal{O}(\Gamma))}\left(M, A_{\lambda}^{*}(N)\right) .
\end{aligned}
$$

Proof. This is obvious because the functors ${ }^{\mathcal{O}} \operatorname{Ind}_{n, m r}$ and ${ }^{\mathcal{O}} \operatorname{Res}_{n, m r}$ are exact and biadjoint; see [2], 33]. 
5.2. The $\mathfrak{S}_{r}$-action on $\left(A_{!}\right)^{r},\left(A^{*}\right)^{r}$ and $\left(A_{*}\right)^{r}$. For $b=!$, * we write $A^{*}=A_{(1)}^{*}$ and $A_{b}=A_{(1), b}$. For $r \geqslant 1$, the transitivity of the induction and restriction functors [33, cor. 2.5] yield functor isomorphisms:

$$
\begin{aligned}
\left(A_{!}\right)^{r} & =\operatorname{RHom}_{D^{b}\left(\mathcal{O}\left(\mathfrak{S}_{\left(m^{r}\right)}\right)\right)}\left({ }^{\mathcal{O}} \operatorname{Res}_{n,\left(m^{r}\right)}(\bullet), L\right)^{\circledast}, \\
\left(A^{*}\right)^{r} & ={ }^{\mathcal{O}} \operatorname{Ind}_{n,\left(m^{r}\right)}(\bullet \otimes L)={ }^{\mathcal{O}} \operatorname{Ind}_{n, m r}\left(\bullet \otimes \mathcal{O} \operatorname{Ind}_{\left(m^{r}\right)}(L)\right), \\
\left(A_{*}\right)^{r} & =\operatorname{RHom}_{D^{b}\left(\mathcal{O}\left(\mathfrak{S}_{\left(m^{r}\right)}\right)\right.}\left(L,{ }^{\mathcal{O}} \operatorname{Res}_{n,\left(m^{r}\right)}(\bullet)\right) .
\end{aligned}
$$

Here, to unburden the notation we abbreviate $L=L_{(m)}^{\otimes r}$. The goal of this section is to construct an $\mathfrak{S}_{r}$-action on $\left(A_{!}\right)^{r},\left(A^{*}\right)^{r}$ and $\left(A_{*}\right)^{r}$, and to decompose these functors using this action. To do this, let $\mathbf{H}\left(\Gamma_{n,\left(m^{r}\right)}\right), \mathbf{H}\left(\Gamma_{n}\right), \mathbf{H}\left(\mathfrak{S}_{m}\right)$ be as in Appendix A, with the parameters $\zeta$ and $v_{p}$ as in Section 3.7. There is an obvious isomorphism

$$
\mathbf{H}\left(\Gamma_{n,\left(m^{r}\right)}\right)=\mathbf{H}\left(\Gamma_{n}\right) \otimes \mathbf{H}\left(\mathfrak{S}_{m}\right)^{\otimes r} .
$$

Let $\tau_{i} \in \mathfrak{S}_{n+m r}$ be the unique permutation such that

- $\tau_{i}$ is minimal in the coset $\mathfrak{S}_{\left(n, m^{r}\right)} \tau_{i} \mathfrak{S}_{\left(n, m^{r}\right)}$,

- $\tau_{i}\left(v w_{1} w_{2} \ldots w_{r}\right) \tau_{i}^{-1}=v w_{1} \ldots w_{i+1} w_{i} \ldots w_{r}$ for $v \in \mathfrak{S}_{n}, w_{1}, \ldots, w_{r} \in \mathfrak{S}_{m}$.

Let $\tau_{i}$ also denote the algebra isomorphism $\mathbf{H}\left(\Gamma_{n,\left(m^{r}\right)}\right) \rightarrow \mathbf{H}\left(\Gamma_{n,\left(m^{r}\right)}\right)$ given by

$$
x \otimes y_{1} \otimes \cdots \otimes y_{r} \rightarrow x \otimes y_{1} \otimes \cdots \otimes y_{i+1} \otimes y_{i} \otimes \cdots \otimes y_{r} .
$$

We have the following relation in $\mathbf{H}\left(\Gamma_{n+m r}\right)$ :

$$
T_{\tau_{i}} z=\tau_{i}(z) T_{\tau_{i}}, \quad z \in \mathbf{H}\left(\Gamma_{n,\left(m^{r}\right)}\right) .
$$

Therefore, the element $T_{\tau_{i}}$ belongs to the normalizer of $\mathbf{H}\left(\Gamma_{n,\left(m^{r}\right)}\right)$ in $\mathbf{H}\left(\Gamma_{n+m r}\right)$.

The twist of a module by $\tau_{i}$ yields the functor

$$
\begin{gathered}
\tau_{i}: \operatorname{Rep}\left(\mathbf{H}\left(\Gamma_{n,\left(m^{r}\right)}\right)\right) \rightarrow \operatorname{Rep}\left(\mathbf{H}\left(\Gamma_{n,\left(m^{r}\right)}\right)\right), \\
M \otimes N_{1} \otimes \cdots \otimes N_{r} \rightarrow M \otimes N_{1} \otimes \cdots \otimes N_{i+1} \otimes N_{i} \otimes \cdots \otimes N_{r} .
\end{gathered}
$$

We define the morphism of functors

$$
\begin{aligned}
& \mathbf{H}_{\tau_{i}:}{ }^{\mathbf{H}} \operatorname{Ind}_{n,\left(m^{r}\right)} \rightarrow{ }^{\mathbf{H}} \operatorname{Ind}_{n,\left(m^{r}\right)} \circ \tau_{i}, \quad \mathbf{H}_{\tau_{i}}(M)(h \otimes v)=h T_{\tau_{i}} \otimes \tau_{i}(v), \\
& h \in \mathbf{H}\left(\Gamma_{n+m r}\right), \quad v \in M, \quad M \in \operatorname{Rep}\left(\mathbf{H}\left(\Gamma_{n,\left(m^{r}\right)}\right)\right) .
\end{aligned}
$$

It is well-defined by (5.2). Next, the permutation $\tau_{i}$ also yields a functor

$$
\begin{gathered}
\tau_{i}: \mathcal{O}\left(\Gamma_{n,\left(m^{r}\right)}\right) \rightarrow \mathcal{O}\left(\Gamma_{n,\left(m^{r}\right)}\right), \\
M \otimes N_{1} \otimes \cdots \otimes N_{r} \rightarrow M \otimes N_{1} \otimes \cdots \otimes N_{i+1} \otimes N_{i} \otimes \cdots \otimes N_{r} .
\end{gathered}
$$

The functor $\mathrm{KZ}$ yields a $\mathbb{C}$-algebra isomorphism [33, lem. 2.4]

$$
\mathrm{KZ}: \operatorname{End}\left({ }^{\mathcal{O}} \operatorname{Ind}_{n,\left(m^{r}\right)}\right) \rightarrow \operatorname{End}\left(\mathrm{KZ}_{\circ}{ }^{\mathcal{O}} \operatorname{Ind}_{n,\left(m^{r}\right)}\right)=\operatorname{End}\left({ }^{\mathbf{H}} \operatorname{Ind}_{n,\left(m^{r}\right)} \circ \mathrm{KZ}\right) .
$$

For the same reason we have also an isomorphism

$$
\begin{aligned}
\mathrm{KZ}: & \operatorname{Hom}\left({ }^{\mathcal{O}} \operatorname{Ind}_{n,\left(m^{r}\right)},{ }^{\mathcal{O}} \operatorname{Ind}_{n,\left(m^{r}\right)} \circ \tau_{i}\right) \\
& \rightarrow \operatorname{Hom}\left({ }^{\mathbf{H}} \operatorname{Ind}_{n,\left(m^{r}\right)} \circ \mathrm{KZ},{ }^{\mathbf{H}} \operatorname{Ind}_{n,\left(m^{r}\right)} \circ \tau_{i} \circ \mathrm{KZ}\right) .
\end{aligned}
$$

So there is a unique morphism of functors

$$
\mathcal{O}_{\tau_{i}:}{ }^{\mathcal{O}} \operatorname{Ind}_{n,\left(m^{r}\right)} \rightarrow{ }^{\mathcal{O}} \operatorname{Ind}_{n,\left(m^{r}\right)} \circ \tau_{i}
$$

which satisfies the following identity:

$$
\mathrm{KZ}\left({ }^{\mathcal{O}} \tau_{i}(M)\right)={ }^{\mathbf{H}_{i}} \tau_{i}(\mathrm{KZ}(M)), \quad M \in \mathcal{O}\left(\Gamma_{n,\left(m^{r}\right)}\right) .
$$


The functor $\bullet \otimes L$ yields a map

$$
\operatorname{Hom}\left({ }^{\mathcal{O}} \operatorname{Ind}_{n,\left(m^{r}\right)},{ }^{\mathcal{O}} \operatorname{Ind}_{n,\left(m^{r}\right)} \circ \tau_{i}\right) \rightarrow \operatorname{End}\left(\left(A^{*}\right)^{r}\right) .
$$

Let $\bar{\tau}_{i}$ denote the image of ${ }^{\mathcal{O}} \tau_{i}$ by this map.

Lemma 5.3. The following relations hold in $\operatorname{End}\left(\left(A^{*}\right)^{r}\right)$ :

- $\bar{\tau}_{i}^{2}=1$,

- $\bar{\tau}_{i} \bar{\tau}_{j}=\bar{\tau}_{j} \bar{\tau}_{i}$ if $j \neq i-1, i+1$,

- $\bar{\tau}_{i} \bar{\tau}_{i+1} \bar{\tau}_{i}=\bar{\tau}_{i+1} \bar{\tau}_{i} \bar{\tau}_{i+1}$.

Proof. We'll write $L^{S}=\left(L_{(m)}^{S}\right)^{\dot{\otimes} r}$. Consider the morphism of functors

$$
\begin{gathered}
{ }^{\mathbf{H}} \tau_{i}^{0}:{ }^{\mathbf{H} \operatorname{Ind}_{\left(m^{r}\right)} \rightarrow{ }^{\mathbf{H}} \operatorname{Ind}_{\left(m^{r}\right)} \circ \tau_{i},} \quad{ }^{\mathbf{H}} \tau_{i}^{0}(M)(h \otimes v)=h T_{\tau_{i}} \otimes \tau_{i}(v), \\
h \in \mathbf{H}\left(\mathfrak{S}_{m r}\right), \quad v \in M, \quad M \in \operatorname{Rep}\left(\mathbf{H}\left(\mathfrak{S}_{m}\right){ }^{\otimes r}\right) .
\end{gathered}
$$

It is well-defined by (5.2). By (5.3) there is a unique morphism of functors

$$
{ }^{\mathcal{O}} \tau_{i}^{0}:{ }^{\mathcal{O}} \operatorname{Ind}_{\left(m^{r}\right)} \rightarrow{ }^{\mathcal{O}} \operatorname{Ind}_{\left(m^{r}\right)} \circ \tau_{i}
$$

such that

$$
\mathrm{KZ}\left({ }^{\mathcal{O}} \tau_{i}^{0}(M)\right)={ }^{\mathbf{H}} \tau_{i}^{0}(\mathrm{KZ}(M)) .
$$

We define the endomorphism $\bar{\tau}_{i}^{0}$ of the module ${ }^{\mathcal{O}} \operatorname{Ind}_{\left(m^{r}\right)}(L)$ by

$$
\bar{\tau}_{i}^{0}={ }^{\mathcal{O}} \tau_{i}^{0}(L) .
$$

The transitivity of the induction functor [33, cor. 2.5] yields

$$
\begin{gathered}
\left(A^{*}\right)^{r}(M)={ }^{\mathcal{O}} \operatorname{Ind}_{n, m r}\left(M \otimes{ }^{\mathcal{O}} \operatorname{Ind}_{\left(m^{r}\right)}(L)\right), \\
\bar{\tau}_{i}(M)={ }^{\mathcal{O}} \operatorname{Ind}_{n, m r}\left(\mathbf{1} \otimes \bar{\tau}_{i}^{0}\right) .
\end{gathered}
$$

Therefore, we are reduced to checking the following relations:

- $\left(\bar{\tau}_{i}^{0}\right)^{2}=1$,

- $\bar{\tau}_{i}^{0} \bar{\tau}_{j}^{0}=\bar{\tau}_{j}^{0} \bar{\tau}_{i}^{0}$ if $j \neq i-1, i+1$,

- $\bar{\tau}_{i}^{0} \bar{\tau}_{i+1}^{0} \bar{\tau}_{i}^{0}=\bar{\tau}_{i+1}^{0} \bar{\tau}_{i}^{0} \bar{\tau}_{i+1}^{0}$.

To prove this, recall that Rouquier's functor $R$ yields an equivalence

$$
\mathcal{O}\left(\mathfrak{S}_{m r}\right) \rightarrow \operatorname{Rep}\left(\mathbf{S}_{\zeta}(m r)\right)
$$

Here $\zeta$ is a primitive $m$-th root of 1 . We have

$$
R\left(L_{m \lambda}\right)=L_{m \lambda}^{S}
$$

By Proposition 3.1 we also have

$$
R\left({ }^{\mathcal{O}} \operatorname{Ind}_{\left(m^{r}\right)}(L)\right)=L^{S} .
$$

Thus the functor $R$ yields a $\mathbb{C}$-algebra isomorphism

$$
\operatorname{End}_{\mathcal{O}\left(\mathfrak{S}_{m r}\right)}\left({ }^{\mathcal{O}} \operatorname{Ind}_{\left(m^{r}\right)}(L)\right)=\operatorname{End}_{\mathbf{S}_{\zeta}(m r)}\left(L^{S}\right) .
$$

Therefore, we are reduced to checking the following relations in $\operatorname{End}_{\mathbf{S}_{\zeta}(m r)}\left(L^{S}\right)$ :

- $R\left(\bar{\tau}_{i}^{0}\right)^{2}=1$,

- $R\left(\bar{\tau}_{i}^{0}\right) R\left(\bar{\tau}_{j}^{0}\right)=R\left(\bar{\tau}_{j}^{0}\right) R\left(\bar{\tau}_{i}^{0}\right)$ if $j \neq i-1, i+1$,

- $R\left(\bar{\tau}_{i}^{0}\right) R\left(\bar{\tau}_{i+1}^{0}\right) R\left(\bar{\tau}_{i}^{0}\right)=R\left(\bar{\tau}_{i+1}^{0}\right) R\left(\bar{\tau}_{i}^{0}\right) R\left(\bar{\tau}_{i+1}^{0}\right)$. 
By Proposition 3.1 there is an isomorphism of functors $\mathcal{O}\left(\mathfrak{S}_{\left(m^{r}\right)}\right) \rightarrow \operatorname{Rep}\left(\mathbf{S}_{\zeta}(m r)\right)$ :

$$
(\bullet)^{\dot{\otimes}} \circ R=R \circ{ }^{\mathcal{O}} \operatorname{Ind}_{\left(m^{r}\right)} \circ(\bullet)^{\otimes r} .
$$

Since $\mathbf{1}_{R} \mathcal{O}_{\tau_{i}^{0}} \mathbf{1}_{(\bullet) \otimes r}$ is an endomorphism of the right-hand side and since $R$ is an equivalence, there is a unique endomorphism ${ }^{\mathbf{s}} \tau_{i}^{0}$ of the functor

$$
(\bullet)^{\dot{\otimes} r}: \operatorname{Rep}\left(\mathbf{S}_{\zeta}(m)\right) \rightarrow \operatorname{Rep}\left(\mathbf{S}_{\zeta}(m r)\right)
$$

such that

$$
\mathbf{s}_{\tau_{i}^{0}} \mathbf{1}_{R}=\mathbf{1}_{R} \mathcal{O}_{i}^{0} \mathbf{1}_{(\bullet) \otimes r}
$$

Consider the diagram

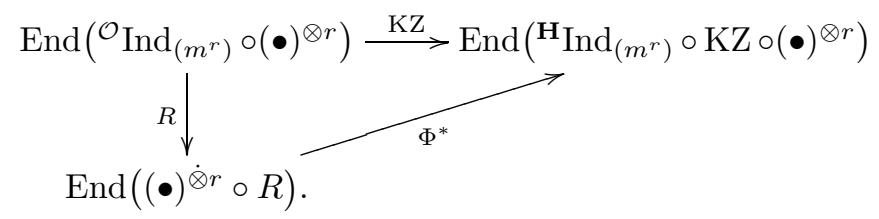

The upper map is invertible by (5.3), the vertical one by Proposition 3.1 and the lower one by Corollary B.4. The diagram is commutative because $\Phi^{*} \circ R=\mathrm{KZ}$. By (5.7) and (5.12) the image of $\mathcal{O}_{i}^{0} \mathbf{1}_{(\bullet) \otimes r}$ is given by

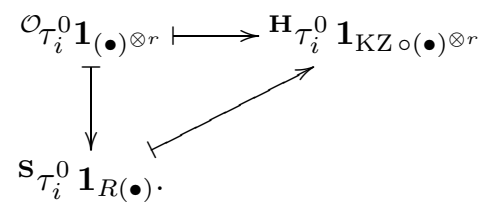

Now, recall the endomorphisms of functors $\mathcal{R}_{\bullet}, i, \mathcal{S}_{\bullet}, i$ defined in (B.10), (B.9). By Corollary B.7 the functor $\Phi^{*}$ yields a map

$$
\operatorname{End}\left((\bullet)^{\otimes r}\right) \rightarrow \operatorname{End}\left({ }^{\mathbf{H}} \operatorname{Ind}_{\left(m^{r}\right)} \circ(\bullet)^{\otimes r} \circ \Phi^{*}\right), \quad \mathcal{R}_{\bullet, i} \mapsto \mathcal{S}_{\Phi^{*}(\bullet), i}
$$

By (5.6) we have

$$
\mathcal{S}_{M, i}={ }^{\mathbf{H}} \tau_{i}^{0}\left(M^{\otimes r}\right), \quad M \in \operatorname{Rep}\left(\mathbf{H}\left(\mathfrak{S}_{m}\right)\right) .
$$

Therefore, by (5.13) we also have

$$
\mathcal{R}_{M, i}={ }^{\mathbf{s}} \tau_{i}^{0}(M), \quad M \in \operatorname{Rep}\left(\mathbf{S}_{\zeta}(m)\right) .
$$

Now, by (5.8), (5.11) and (5.12) we have

$$
R\left(\bar{\tau}_{i}^{0}\right)={ }^{\mathbf{s}} \tau_{i}^{0}\left(L_{(m)}^{S}\right) .
$$

Thus, by (5.14) we must check that the operators $\mathcal{R}_{L_{(m)}^{S}, i}$ satisfy the same relations as above. The quantum Frobenius homomorphism yields a functor

$$
\operatorname{Fr}^{*}: \operatorname{Rep}\left(\mathbf{S}_{1}(r)\right) \rightarrow \operatorname{Rep}\left(\mathbf{S}_{\zeta}(m r)\right)
$$

such that $L_{(m)}^{S}=\operatorname{Fr}^{*}\left(\bar{L}_{(1)}^{S}\right)$; see Section B.7. It is a braided tensor functor by Proposition B.9. Thus the claim follows from Proposition B.8.

We can now prove the following, which is the main result of this subsection. 
Proposition 5.4. Let $r \geqslant 1$.

(a) The group $\mathfrak{S}_{r}$ acts on the functors $\left(A^{*}\right)^{r},\left(A_{*}\right)^{r}$.

(b) We have the following $\mathfrak{S}_{r}$-equivariant isomorphisms of functors:

$$
\left(A^{*}\right)^{r}=\bigoplus_{\lambda \in \mathcal{P}_{r}} \bar{L}_{\lambda} \otimes A_{\lambda}^{*}, \quad\left(A_{*}\right)^{r}=\bigoplus_{\lambda \in \mathcal{P}_{r}} \bar{L}_{\lambda} \otimes A_{\lambda, *} .
$$

Proof. First, we concentrate on part $(a)$. To unburden the notation we abbreviate

$$
L=L_{(m)}^{\otimes r}, \quad L^{S}=\left(L_{(m)}^{S}\right)^{\dot{\otimes} r} .
$$

By Lemma 5.3 the assignment $s_{i} \mapsto \bar{\tau}_{i}$ yields a $\mathfrak{S}_{r}$-action on $\left(A^{*}\right)^{r}$. Under the adjunction $\left({ }^{\mathcal{O}} \operatorname{Ind}_{n,\left(m^{r}\right)},{ }^{\mathcal{O}} \operatorname{Res}_{n,\left(m^{r}\right)}\right)$ the isomorphism $\mathcal{O}_{\tau_{i}}$ yields a (right transposed) isomorphism of ${ }^{\mathcal{O}} \operatorname{Res}_{n,\left(m^{r}\right)}$. We'll denote it by ${ }^{\mathcal{O}} \tau_{i}$ again. By definition of the right transposition, the following square is commutative for $M \in \mathcal{O}\left(\Gamma_{n+m r}\right)$ :

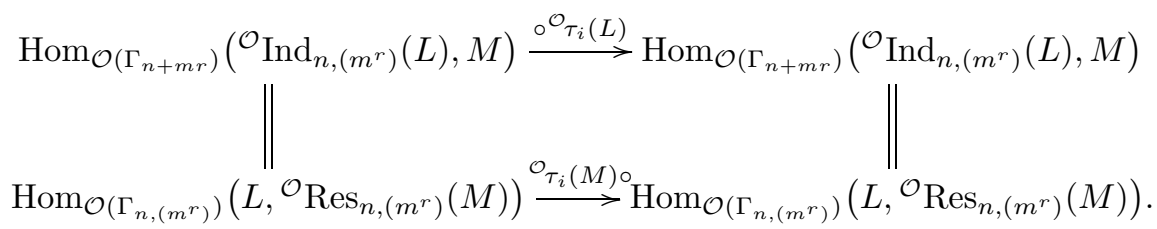

Here and in the rest of the proof, we use the canonical isomorphisms

$$
\begin{aligned}
& \operatorname{Hom}_{\mathcal{O}\left(\Gamma_{n+m r}\right)}\left({ }^{\mathcal{O}} \operatorname{Ind}_{n,\left(m^{r}\right)}(L), M\right)=\operatorname{Hom}_{\mathcal{O}\left(\Gamma_{n+m r}\right)}\left({ }^{\mathcal{O}} \operatorname{Ind}_{n,\left(m^{r}\right)}\left(\tau_{i}(L)\right), M\right), \\
& \operatorname{Hom}_{\mathcal{O}\left(\Gamma_{n,\left(m^{r}\right)}\right)}\left(L,{ }^{\mathcal{O}} \operatorname{Res}_{n,\left(m^{r}\right)}(M)\right)=\operatorname{Hom}_{\mathcal{O}\left(\Gamma_{n,\left(m^{r}\right)}\right)}\left(L, \tau_{i}\left({ }^{\mathcal{O}} \operatorname{Res}_{n,\left(m^{r}\right)}(M)\right)\right)
\end{aligned}
$$

given by $\tau_{i}(L)=L$ without mentionning them explicitly. We define the $\mathfrak{S}_{r}$-action on $\left(A_{*}\right)^{r}$ by

$$
\begin{gathered}
s_{i}(f)={ }^{\mathcal{O}} \tau_{i}(M) \circ f \\
f \in\left(A_{*}\right)^{r}(M)=\operatorname{RHom}_{D^{b}\left(\mathcal{O}\left(\mathfrak{S}_{\left(m^{r}\right)}\right)\right)}\left(L,{ }^{\mathcal{O}} \operatorname{Res}_{n,\left(m^{r}\right)}(M)\right) .
\end{gathered}
$$

Note that the formulas (5.15) do define an action of the group $\mathfrak{S}_{r}$ by Lemma 5.3 . because the square above is commutative.

Now, we prove part $(b)$. It is convenient to rewrite the $\mathfrak{S}_{r}$-action on $\left(A^{*}\right)^{r}$ in a slightly different way. Setting $n=0$ in the construction above we get a $\mathfrak{S}_{r}$-action on ${ }^{\mathcal{O}} \operatorname{Ind}_{\left(m^{r}\right)}(L)$ such that $s_{i}$ acts through the operator $\bar{\tau}_{i}^{0}$ in (5.8), and by (5.9) the reflection $s_{i}$ acts on $\left(A^{*}\right)^{r}$ through the automorphism

$$
{ }^{\mathcal{O}} \operatorname{Ind}_{n, m r}\left(\mathbf{1} \otimes \mathcal{O}_{\bar{\tau}_{i}^{0}}^{0}\right) \text {. }
$$

We claim that the following identity holds in $\operatorname{Rep}\left(\mathbb{C S}_{r}\right) \otimes \mathcal{O}\left(\mathfrak{S}_{m r}\right)$ :

$$
{ }^{\mathcal{O}} \operatorname{Ind}_{\left(m^{r}\right)}(L)=\bigoplus_{\lambda \in \mathcal{P}_{r}} \bar{L}_{\lambda} \otimes L_{m \lambda}
$$

To prove (5.16) we use Rouquier's functor $R$ as in the proof of Lemma 5.3, It is enough to check the following identity in $\operatorname{Rep}\left(\mathbb{C S}_{r}\right) \otimes \operatorname{Rep}\left(\mathbf{S}_{\zeta}(m r)\right)$ :

$$
L^{S}=\bigoplus_{\lambda \in \mathcal{P}_{r}} \bar{L}_{\lambda} \otimes L_{m \lambda}^{S}
$$

To do that, note that by Proposition B.9 the functor in Section B.7.

$$
\operatorname{Fr}^{*}: \operatorname{Rep}\left(\mathbf{S}_{1}(r)\right)=\operatorname{Rep}\left(\mathbf{S}_{(-1)^{m}}(r)\right) \rightarrow \operatorname{Rep}\left(\mathbf{S}_{\zeta}(m r)\right),
$$


given by the quantum Frobenius homomorphism, is a braided tensor functor. Further we have

$$
\operatorname{Fr}^{*}\left(\bar{L}_{\lambda}^{S}\right)=L_{m \lambda}^{S}, \quad \operatorname{Fr}^{*}\left(\left(\bar{L}_{(1)}^{S}\right)^{\dot{\otimes} r}\right)=L^{S},
$$

where $\bar{L}_{\lambda}^{S}$ is the simple $\mathbf{S}_{1}(r)$-module with the highest weight $\lambda$. Therefore, to prove (5.16) we are reduced to checking the following identity in $\operatorname{Rep}\left(\mathbb{C S}_{r}\right) \otimes \operatorname{Rep}\left(\mathbf{S}_{1}(r)\right)$ :

$$
\left(\bar{L}_{(1)}^{S}\right)^{\dot{\otimes} r}=\bigoplus_{\lambda \in \mathcal{P}_{r}} \bar{L}_{\lambda} \otimes \bar{L}_{\lambda}^{S}
$$

This is a trivial consequence of the Schur duality. The decomposition

$$
\left(A^{*}\right)^{r}=\bigoplus_{\lambda \in \mathcal{P}_{r}} \bar{L}_{\lambda} \otimes A_{\lambda}^{*}
$$

is a direct consequence of (5.16). The decomposition of the functor $\left(A_{*}\right)^{r}$ follows from (5.16) and the commutativity of the diagram above, because it implies that the canonical isomorphism

$$
\left(A_{*}\right)^{r}(M)=\operatorname{RHom}_{D^{b}\left(\mathcal{O}\left(\mathfrak{S}_{\left(m^{r}\right)}\right)\right)}\left({ }^{\mathcal{O}} \operatorname{Ind}_{\left(m^{r}\right)}(L),{ }^{\mathcal{O}} \operatorname{Res}_{n, m r}(M)\right)
$$

is $\mathfrak{S}_{r}$-equivariant.

Remark 5.5. Using an adjunction $\left({ }^{\mathcal{O}} \operatorname{Res}_{n,\left(m^{r}\right)},{ }^{\mathcal{O}} \operatorname{Ind}_{n,\left(m^{r}\right)}\right)$ for each $r$, we can construct in a similar way a $\mathfrak{S}_{r}$-action on the functor $\left(A_{!}\right)^{r}$ such that we have the decomposition

$$
\left(A_{!}\right)^{r}=\bigoplus_{\lambda \in \mathcal{P}_{r}} \bar{L}_{\lambda} \otimes A_{\lambda, !}
$$

Then, by Propositions 5.2 and 5.4, we have the triple $\left(\left(A_{!}\right)^{r},\left(A^{*}\right)^{r},\left(A_{*}\right)^{r}\right)$ of adjoint $\mathfrak{S}_{r}$-equivariant functors.

Remark 5.6. We have used the hypothesis $m>2$ in the proof of Proposition 5.4 when using Rouquier's functor $R$. Probably this is not necessary.

Proposition 5.7. For $r \geqslant 1$ we have an isomorphism of functors

$$
\left(A_{!}\right)^{r}[2 r(1-m)]=\left(A_{*}\right)^{r} .
$$

Proof. Once again we'll abbreviate $L=L_{(m)}^{\otimes r}$. Let $\mathcal{P} \operatorname{erv}\left(\mathbb{P}^{m-1}\right)$ be the category of perverse sheaves on $\mathbb{P}^{m-1}$ which are constructible with respect to the standard stratification $\mathbb{P}^{m-1}=\mathbb{C}^{0} \cup \mathbb{C}^{1} \cup \cdots \cup \mathbb{C}^{m-1}$. By [3, thm. 1.3] the category $\mathcal{O}\left(\mathfrak{S}_{m}\right)$ decomposes as the direct sum of $\operatorname{Perv}\left(\mathbb{P}^{m-1}\right)$ and semisimple blocks. Under this equivalence the module $L_{(m)}$ is taken to be the perverse sheaf $\mathbb{C}_{\mathbb{P}^{m-1}}[m-1]$. So, by Verdier duality [20, (3.1.8)], we have an isomorphism of functors from $D^{b}\left(\mathcal{O}\left(\mathfrak{S}_{m}\right)\right.$ ) to $D^{b}(\mathbb{C})$ :

$$
\operatorname{RHom}_{D^{b}\left(\mathcal{O}\left(\mathfrak{S}_{m}\right)\right)}\left(L_{(m)}, \bullet\right) \rightarrow \operatorname{RHom}_{D^{b}\left(\mathcal{O}_{\left(\mathfrak{S}_{m}\right)}\right)}\left(\bullet, L_{(m)}\right)^{*}[2(1-m)] .
$$

The tensor power of (5.18) is an isomorphism of functors $D^{b}\left(\mathcal{O}\left(\mathfrak{S}_{\left(m^{r}\right)}\right)\right) \rightarrow D^{b}(\mathbb{C})$ :

$$
\theta^{0}: \operatorname{RHom}_{D^{b}\left(\mathcal{O}\left(\mathfrak{S}_{\left(m^{r}\right)}\right)\right)}(L, \bullet) \rightarrow \operatorname{RHom}_{D^{b}\left(\mathcal{O}\left(\mathfrak{S}_{\left(m^{r}\right)}\right)\right)}(\bullet, L)^{*}[2 r(1-m)] .
$$

It yields an isomorphism of functors $D^{b}\left(\mathcal{O}\left(\Gamma_{n,\left(m^{r}\right)}\right)\right) \rightarrow D^{b}\left(\mathcal{O}\left(\Gamma_{n}\right)\right)$ :

$$
\theta: \operatorname{RHom}_{D^{b}\left(\mathcal{O}\left(\mathfrak{S}_{\left(m^{r}\right)}\right)\right)}(L, \bullet) \rightarrow \operatorname{RHom}_{D^{b}\left(\mathcal{O}_{\left.\left(\mathfrak{S}_{\left(m^{r}\right)}\right)\right)}(\bullet, L)\right.}{ }^{\circledast}[2 r(1-m)]
$$


such that

$$
\begin{gathered}
\theta\left(\tau_{i}(M)\right)\left(\tau_{i}(f)\right)=\tau_{i}(\theta(M)(f)), \\
M \in \mathcal{O}\left(\Gamma_{n,\left(m^{r}\right)}\right), \quad f \in \operatorname{RHom}_{D^{b}\left(\mathcal{O}\left(\mathfrak{S}_{\left(m^{r}\right)}\right)\right)}(L, M) .
\end{gathered}
$$

We define an isomorphism of functors $D^{b}\left(\mathcal{O}\left(\Gamma_{n+m r}\right)\right) \rightarrow D^{b}\left(\mathcal{O}\left(\Gamma_{n}\right)\right)$ by

$$
\theta^{\prime}=\theta \mathbf{1}_{\mathcal{O} \operatorname{Res}_{n,\left(m^{r}\right)}} .
$$

More precisely, we have

$$
\begin{gathered}
\theta^{\prime}: \operatorname{RHom}_{D^{b}\left(\mathcal{O}\left(\mathfrak{S}_{\left(m^{r}\right)}\right)\right)}\left(L,{ }^{\mathcal{O}} \operatorname{Res}_{n,\left(m^{r}\right)}(\bullet)\right) \\
\rightarrow \operatorname{RHom}_{D^{b}\left(\mathcal{O}\left(\mathfrak{S}_{\left(m^{r}\right)}\right)\right)}\left({ }^{\mathcal{O}} \operatorname{Res}_{n,\left(m^{r}\right)}(\bullet), L\right)^{\circledast}[2 r(1-m)] .
\end{gathered}
$$

By (5.1) we may view $\theta^{\prime}$ as an isomorphism $\left(A_{*}\right)^{r} \rightarrow\left(A_{!}\right)^{r}[2 r(1-m)]$.

Remark 5.8. Probably we can choose the $\mathfrak{S}_{r}$-action on $\left(A_{!}\right)^{r}$ in such a way that the isomorphism $\left(A_{*}\right)^{r} \rightarrow\left(A_{!}\right)^{r}[2 r(1-m)]$ is $\mathfrak{S}_{r}$-equivariant. This would imply that for $\lambda \in \mathcal{P}_{r}$ we have $A_{\lambda, !}[2 r(1-m)]=A_{\lambda, *}$. We'll not use this.

Remark 5.9. The transitivity of the induction functor [33, cor. 2.5] yields an isomorphism of functors $A_{\lambda}^{*} A_{\mu}^{*}=A_{\mu}^{*} A_{\lambda}^{*}$ for $\lambda, \mu \in \mathcal{P}$. Taking the adjoint functors we also get the isomorphisms $A_{\lambda, !} A_{\mu, !}=A_{\mu, !} A_{\lambda, !}$ and $A_{\lambda, *} A_{\mu, *}=A_{\mu, *} A_{\lambda, *}$.

Remark 5.10. The functors $A_{\lambda, !}, A_{\lambda}^{*}, A_{\lambda, *}$ yield linear endomorphisms of the $\mathbb{C}$ vector space $[\mathcal{O}(\Gamma)]$. Let us denote them by $A_{\lambda, !}, A_{\lambda}^{*}, A_{\lambda, *}$ again.

Remark 5.11. Recall that $\langle m\rangle=\bigoplus_{i=0}^{m-1} \mathbb{C}[-2 i]$. For any object $M$ of $D^{b}(\mathcal{O}(\Gamma))$ there should be a distinguished triangle

$$
\ell\langle m\rangle M \longrightarrow A_{*} A^{*}(M) \longrightarrow A^{*} A_{*}(M) \stackrel{+1}{\longrightarrow} .
$$

5.3. The functors $a_{\lambda}^{*}, a_{\lambda, *}$ on $\mathcal{O}(\Gamma)$ and the $\mathfrak{H}$-action on the Fock space. For $i \in \mathbb{Z}$ and $b=$ !, * we consider the endofunctor $H^{i}\left(A_{\lambda, b}\right)$ of $\mathcal{O}(\Gamma)$ given by

$$
H^{i}\left(A_{\lambda, b}\right)(M)=H^{i}\left(A_{\lambda, b}(M)\right), \quad M \in \mathcal{O}(\Gamma) .
$$

From now on we'll write $R a_{\lambda, b}=A_{\lambda, b}$ and $R^{i} a_{\lambda, b}=H^{i}\left(A_{\lambda, b}\right)$.

Definition 5.12. Let $a_{\lambda}^{*}$ be the restriction of $A_{\lambda}^{*}$ to the Abelian category $\mathcal{O}(\Gamma)$. Since $a_{\lambda}^{*}$ is an exact endofunctor of $\mathcal{O}(\Gamma)$, we may write $a_{\lambda}^{*}$ for $A_{\lambda}^{*}$ if it does not create any confusion. We abbreviate $a_{\lambda, b}=R^{0} a_{\lambda, b}$. The functor $a_{\lambda, *}$ is a left exact endofunctor of $\mathcal{O}(\Gamma)$, while $a_{\lambda, !}$ is right exact.

Consider the chain of $\mathbb{C}$-linear isomorphisms which is the composition of (3.5), of the characteristic map ch, and of (4.19),

$$
\begin{aligned}
& {[\mathcal{O}(\Gamma)] \rightarrow R(\Gamma) \rightarrow \Lambda_{\Gamma} \rightarrow \mathcal{F}_{m, \ell}^{(s)},} \\
& \Delta_{\lambda} \mapsto \bar{L}_{\lambda} \quad \mapsto S_{\tau \lambda} \mapsto|\lambda, s\rangle .
\end{aligned}
$$

Recall the symmetric bilinear form on $\mathcal{F}_{m, \ell}^{(s)}$ defined in Section 4.6.

Proposition 5.13. (a) The map (5.20) identifies the symmetric $\mathbb{C}$-bilinear form on $\mathcal{F}_{m, \ell}^{(s)}$ with the $\mathbb{C}$-bilinear form

$$
[\mathcal{O}(\Gamma)] \times[\mathcal{O}(\Gamma)] \rightarrow \mathbb{C}, \quad(M, N) \mapsto \sum_{i}(-1)^{i} \operatorname{dim}_{\operatorname{Ext}_{\mathcal{O}(\Gamma)}^{i}}(M, N)
$$


(b) The map (5.20) identifies the operators $b_{S_{\lambda}}, b_{S_{\lambda}}^{\prime}$ on $\mathcal{F}_{m, \ell}^{(s)}$ with the operators $a_{\lambda}^{*}, R a_{\lambda, *}$ on $[\mathcal{O}(\Gamma)]$.

Proof. Part $(a)$ is obvious because we have

$$
\operatorname{dim} \operatorname{Ext}_{\mathcal{O}\left(\Gamma_{n}\right)}^{i}\left(\Delta_{\lambda}, \nabla_{\mu}\right)=\delta_{i, 0} \delta_{\lambda, \mu}, \quad\left[\Delta_{\mu}\right]=\left[\nabla_{\mu}\right], \quad \forall \lambda, \mu \in \mathcal{P}_{n}^{\ell},
$$

because $\mathcal{O}\left(\Gamma_{n}\right)$ is a quasi-hereditary category; see, e.g., [9, prop. A.2.2]. Now we concentrate on $(b)$. By $(a)$ and Proposition 5.2, the pairs $\left(b_{S_{\lambda}}, b_{S_{\lambda}}^{\prime}\right)$ and $\left(a_{\lambda}^{*}, R a_{\lambda, *}\right)$ consist of adjoint linear operators on $\mathcal{F}_{m, \ell}^{(s)}$. So it is enough to check that under (5.20) we have the following equality:

$$
b_{S_{\lambda}}=a_{\lambda}^{*} .
$$

To do that, observe first that, by Proposition 4.1, for $r>0$ the map ch : $R(\Gamma) \rightarrow \boldsymbol{\Lambda}_{\Gamma}$ intertwines the operator

$$
R(\Gamma) \rightarrow R(\Gamma), \quad M \mapsto \operatorname{Ind}_{\Gamma \times \mathfrak{S}}^{\Gamma}\left(M \otimes \mathrm{ch}^{-1}\left(P_{m r}\right)\right)
$$

and the multiplication by $\sum_{p \in \mathbb{Z}_{\ell}} P_{m r, p}$. Here we have abbreviated

$$
\operatorname{Ind}_{\Gamma \times \mathfrak{S}}^{\Gamma}=\bigoplus_{n, r \geqslant 0} \operatorname{Ind}_{n, m r} .
$$

Next, by Proposition 4.6, the map $\Lambda_{\Gamma} \rightarrow \mathcal{F}_{m, \ell}^{(s)}$ above intertwines the multiplication by $\sum_{p \in \mathbb{Z}_{\ell}} P_{m r, p}$ and the operator $b_{r}$. By definition, the plethysm with the power sum $P_{m}$ is the $\mathbb{C}$-algebra endomorphism

$$
\psi^{m}: \boldsymbol{\Lambda} \rightarrow \boldsymbol{\Lambda}, \quad f \mapsto \sum_{\lambda \in \mathcal{P}} z_{\lambda}^{-1}\left\langle f, P_{\lambda}\right\rangle P_{m \lambda}
$$

The discussion above implies that the map $R(\Gamma) \rightarrow \mathcal{F}_{m, \ell}^{(s)}$ above identifies the action of $b_{S_{\lambda}}$ on $\mathcal{F}_{m, \ell}^{(s)}$ with the operator

$$
R(\Gamma) \rightarrow R(\Gamma), \quad M \mapsto \operatorname{Ind}_{\Gamma \times \mathfrak{S}}^{\Gamma}\left(M \otimes \operatorname{ch}^{-1} \psi^{m}\left(S_{\lambda}\right)\right) .
$$

Now, recall the maps

$$
\text { spe }:\left[\operatorname{Rep}\left(\mathbb{C} \Gamma_{n}\right)\right] \rightarrow\left[\mathcal{O}\left(\Gamma_{n}\right)\right], \quad \text { spe }:\left[\operatorname{Rep}\left(\mathbb{C S}_{m r}\right)\right] \rightarrow\left[\mathcal{O}\left(\mathfrak{S}_{m r}\right)\right] .
$$

By Lemma 2.4, they commute with the induction and restriction. We claim that

$$
\text { spe } \circ \mathrm{ch}^{-1} \circ \psi^{m}\left(S_{\lambda}\right)=L_{m \lambda} .
$$

Thus (5.21) follows from (5.20). To prove the claim, set $\zeta$ equal to a primitive $m$-th root of 1. Then Rouquier's functor yields an isomorphism, see (5.10),

$$
\left[\mathcal{O}\left(\mathfrak{S}_{m r}\right)\right]=\left[\operatorname{Rep}\left(\mathbf{S}_{\zeta}(m r)\right)\right] .
$$

Next, the quantum Frobenius homomorphism yields a commutative diagram

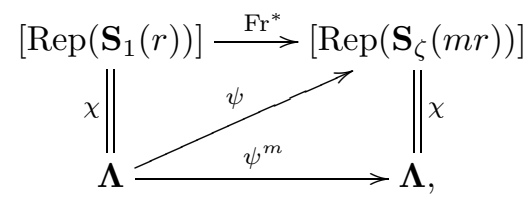


where $\chi$ is the formal character; see, e.g., [16, sec. II.H.9]. Consider the chain of maps

$$
\theta: \quad\left[\operatorname{Rep}\left(\mathbb{C S}_{m r}\right)\right] \stackrel{3.5}{=}\left[\mathcal{O}\left(\mathfrak{S}_{m r}\right)\right] \stackrel{5.10}{=}\left[\operatorname{Rep}\left(\mathbf{S}_{\zeta}(m r)\right)\right]
$$

We have

$$
\psi\left(S_{\lambda}\right)=L_{m \lambda}^{S}, \quad \theta \operatorname{ch}^{-1}\left(S_{\mu}\right)=\Delta_{\mu}^{S}, \quad \lambda \in \mathcal{P}_{r}, \quad \mu \in \mathcal{P}_{m r}
$$

Thus we have

$$
\chi\left(\theta \operatorname{ch}^{-1}\left(S_{\mu}\right)\right)=\chi\left(\Delta_{\mu}^{S}\right)=S_{\mu}, \quad \mu \in \mathcal{P}_{m r} .
$$

Therefore we also have

$$
\chi\left(\theta \circ \mathrm{ch}^{-1} \circ \psi^{m}\left(S_{\lambda}\right)\right)=\psi^{m}\left(S_{\lambda}\right)=\chi\left(\psi\left(S_{\lambda}\right)\right)=\chi\left(L_{m \lambda}^{S}\right) .
$$

This implies that $\theta \circ \mathrm{ch}^{-1} \circ \psi^{m}\left(S_{\lambda}\right)=L_{m \lambda}^{S}$, proving the claim and the proposition.

Remark 5.14. It has been conjectured in [11, sec. 6.6] that the Shapovalov form on $V_{\omega_{d \bmod \ell}}^{\widehat{\mathfrak{g}}_{\ell}}$ should be related to the bilinear form on $[\mathcal{O}(\Gamma)]$ in Proposition 5.13 . Recall that

$$
\mathcal{F}_{\ell}^{(d)}=V_{\omega_{d \bmod \ell}}^{\widehat{\mathfrak{g l}}_{\ell}}
$$

and that the Shapovalov form on the right-hand side is identified with the symmetric bilinear form on the left-hand side considered in Section 4.5. Further, the isomorphism of vector spaces

$$
\mathcal{F}_{\ell}^{(d)}=\mathcal{F}_{m, \ell}^{(d)}
$$

defined by the bijection (4.20) identifies the bilinear form on the left-hand side with the bilinear form on $\mathcal{F}_{m, \ell}^{(d)}$ in Section 4.6. Thus Proposition 5.13 implies Etingof's conjecture.

Proposition 5.15. Let $\lambda \in \mathcal{P}_{r}$ with $r \geqslant 0$.

(a) We have a triple of adjoint functors $\left(a_{\lambda, !}, a_{\lambda}^{*}, a_{\lambda, *}\right)$.

(b) For $b=*, !, q=0,1, \ldots, m-1$, and $i \geqslant 0$ there are isomorphisms of functors

$$
e_{q} R^{i} a_{\lambda, b}=R^{i} a_{\lambda, b} e_{q}, \quad e_{q} a_{\lambda}^{*}=a_{\lambda}^{*} e_{q}, \quad f_{q} R^{i} a_{\lambda, b}=R^{i} a_{\lambda, b} f_{q}, \quad f_{q} a_{\lambda}^{*}=a_{\lambda}^{*} f_{q} .
$$

Proof. By definition of the functors $A_{\lambda, *}, A_{\lambda, !}$ we have

$$
A_{\lambda, *}(\mathcal{O}(\Gamma)) \subset D^{\geqslant 0}(\mathcal{O}(\Gamma)), \quad A_{\lambda, !}(\mathcal{O}(\Gamma)) \subset D^{\leqslant 0}(\mathcal{O}(\Gamma)) .
$$

Thus, by Proposition 5.2 we have the triple of adjoint endofunctors of $\mathcal{O}(\Gamma)$ :

$$
\left(a_{\lambda, !}, a_{\lambda}^{*}, a_{\lambda, *}\right)=\left(H^{0}\left(A_{\lambda, !}\right), A_{\lambda}^{*}, H^{0}\left(A_{\lambda, *}\right)\right) .
$$

This proves $(a)$. Next, let us prove part $(b)$. It is enough to give isomorphisms of functors

$$
e_{q} a_{\lambda}^{*}=a_{\lambda}^{*} e_{q}, \quad f_{q} a_{\lambda}^{*}=a_{\lambda}^{*} f_{q} .
$$

Recall the functor $F$ defined in Section 3.9, First, observe that we have an isomorphism of functors

$$
F a_{\lambda}^{*}=a_{\lambda}^{*} F
$$


Indeed, for $M \in \mathcal{O}\left(\Gamma_{n}\right)$, the transitivity of the induction functor [33, cor. 2.5] yields

$$
\begin{aligned}
F a_{\lambda}^{*}(M) & ={ }^{\mathcal{O}} \operatorname{Ind}_{n+m r}{ }^{\mathcal{O}} \operatorname{Ind}_{n, m r}\left(M \otimes L_{m \lambda}\right) \\
& ={ }^{\mathcal{O}} \operatorname{Ind}_{\Gamma_{n+m r}}^{\Gamma_{n+m r+1}} \mathcal{O}_{\operatorname{Ind}_{\Gamma_{n, m r}}^{\Gamma_{n+m r}}\left(M \otimes L_{m \lambda}\right)} \\
& ={ }^{\mathcal{O}} \operatorname{Ind}_{\Gamma_{n, m r} \Gamma_{n+m r+1}}^{\Gamma_{n+m}}\left(M \otimes L_{m \lambda}\right), \\
a_{\lambda}^{*} F(M) & ={ }^{\mathcal{O}} \operatorname{Ind}_{n+1, m r}\left({ }^{\mathcal{O}} \operatorname{Ind}_{n}(M) \otimes L_{m \lambda}\right) \\
& ={ }^{\mathcal{O}} \operatorname{Ind}_{\Gamma_{n+1, m r}}^{\Gamma_{n+m r+1}}\left({ }^{\mathcal{O}} \operatorname{Ind}_{\Gamma_{n}}^{\Gamma_{n+1}}(M) \otimes L_{m \lambda}\right) \\
& ={ }^{\mathcal{O}} \operatorname{Ind}_{\Gamma_{n+1, m r}}^{\Gamma_{n+1}+1} \mathcal{I n d}_{\Gamma_{n, m r}}^{\Gamma_{n+1, m r}}\left(M \otimes L_{m \lambda}\right) \\
& ={ }^{\mathcal{O}} \operatorname{Ind}_{\Gamma_{n, m r}}^{\Gamma_{n+m r+1}}\left(M \otimes L_{m \lambda}\right) .
\end{aligned}
$$

By (5.24) for each $M \in \mathcal{O}\left(\Gamma_{n}\right)$ we have

$$
\bigoplus_{q} f_{q} a_{\lambda}^{*}(M)=\bigoplus_{q} a_{\lambda}^{*} f_{q}(M)
$$

We must prove that we also have an isomorphism $f_{q} a_{\lambda}^{*}(M)=a_{\lambda}^{*} f_{q}(M)$. Let $\mathcal{O}(\Gamma)_{\nu} \subset \mathcal{O}(\Gamma)$ be the full subcategory consisting of the modules whose class is a weight vector of weight $\nu$ of $[\mathcal{O}(\Gamma)]$. Here $\nu$ is any weight of the $\widetilde{\mathfrak{s l}}_{m}$-module $[\mathcal{O}(\Gamma)]$. Recall that

Lemma 5.16. We have the block decomposition $\mathcal{O}(\Gamma)=\bigoplus_{\nu} \mathcal{O}(\Gamma)_{\nu}$, where $\nu$ runs over the set of all weights of the $\tilde{\mathfrak{s l}}_{m}$-module $[\mathcal{O}(\Gamma)]$.

Proof. By [33, lem. 3.1] the image by $\mathrm{KZ}:[\mathcal{O}(\Gamma)] \rightarrow[\operatorname{Rep}(\mathbf{H}(\Gamma))]$ of the class of a standard module is the class of a Specht module. By [24, thm. 2.11] we have a block decomposition

$$
\operatorname{Rep}(\mathbf{H}(\Gamma))=\bigoplus_{\nu} \operatorname{Rep}(\mathbf{H}(\Gamma))_{\nu}
$$

where $\nu$ runs over a set of weights of $\widetilde{\mathfrak{s l}}_{m}$ and the block $\operatorname{Rep}(\mathbf{H}(\Gamma))_{\nu}$ is generated by the constituents of the Specht modules whose classes are the images by KZ of the class of a standard module in $\mathcal{O}(\Gamma)_{\nu}$. In particular, each Specht module belongs to a single block of $\operatorname{Rep}(\mathbf{H}(\Gamma))$. Now, since the standard modules in $\mathcal{O}(\Gamma)$ are indecomposable (they have a simple top), each of them belongs to a single block and any block is generated by the constituents of the standard modules in this block. Finally, by [14], the functor KZ induces a bijection from the blocks of $\mathcal{O}(\Gamma)$ to the blocks of $\operatorname{Rep}(\mathbf{H}(\Gamma))$. Hence two standard modules belong to the same block of $\mathcal{O}(\Gamma)$ if and only if their images by KZ belong to the same block of $\operatorname{Rep}(\mathbf{H}(\Gamma))$. Therefore $\mathcal{O}(\Gamma)_{\nu}$ is a block of $\mathcal{O}(\Gamma)$. This proves the lemma.

Therefore, to prove the isomorphism $f_{q} a_{\lambda}^{*}(M)=a_{\lambda}^{*} f_{q}(M)$ we may assume that $M$ lies in $\mathcal{O}(\Gamma)_{\nu}$. Then $f_{q} a_{\lambda}^{*}(M)$ and $a_{\lambda}^{*} f_{q}(M)$ belong to $\mathcal{O}(\Gamma)_{\nu-\alpha_{q}}$ by Proposition 5.13. Thus the isomorphism above follows from (5.25). The second isomorphism in (5.23) is proved. Next, let us prove that we have an isomorphism of functors

$$
E a_{\lambda}^{*}=a_{\lambda}^{*} E,
$$

where $E$ is the functor defined in Section 3.9 . The first isomorphism in (5.23) follows from (5.26) by a similar argument to the one above. For $M \in \mathcal{O}\left(\Gamma_{n}\right)$ we 
have

$$
\begin{aligned}
& E a_{\lambda}^{*}(M)={ }^{\mathcal{O}} \operatorname{Res}_{n+m r}{ }^{\mathcal{O}} \operatorname{Ind}_{n, m r}\left(M \otimes L_{m \lambda}\right), \\
& a_{\lambda}^{*} E(M)={ }^{\mathcal{O}} \operatorname{Ind}_{n-1, m r}\left({ }^{\mathcal{O}} \operatorname{Res}_{n}(M) \otimes L_{m \lambda}\right) .
\end{aligned}
$$

As above, we abbreviate $L=L_{(m)}^{\otimes r}$. By Proposition 5.4 it is enough to prove that we have a natural isomorphism

${ }^{\mathcal{O}} \operatorname{Res}_{n+m r}{ }^{\mathcal{O}} \operatorname{Ind}_{n, m r}\left(M \otimes{ }^{\mathcal{O}} \operatorname{Ind}_{\left(m^{r}\right)}(L)\right) \rightarrow{ }^{\mathcal{O}} \operatorname{Ind}_{n-1, m r}\left({ }^{\mathcal{O}} \operatorname{Res}_{n}(M) \otimes{ }^{\mathcal{O}} \operatorname{Ind}_{\left(m^{r}\right)}(L)\right)$

that is equivariant with respect to the $\mathfrak{S}_{r}$-action induced by the $\mathfrak{S}_{r}$-action on ${ }^{\mathcal{O}} \operatorname{Ind}_{\left(m^{r}\right)}(L)$ given in (5.16). To see this, note that Proposition A.2 yields the following decomposition of functors:

$$
\begin{gathered}
{ }^{\mathbf{H}} \operatorname{Res}_{n+m r} \circ{ }^{\mathbf{H}} \operatorname{Ind}_{n, m r}=\left({ }^{\mathbf{H}} \operatorname{Ind}_{n-1, m r} \circ\left({ }^{\mathbf{H}} \operatorname{Res}_{n} \otimes \mathbf{1}\right)\right) \\
\oplus\left({ }^{\mathbf{H}} \operatorname{Ind}_{n, m r-1} \circ\left(\mathbf{1} \otimes{ }^{\mathbf{H}} \operatorname{Res}_{m r}\right)\right){ }^{\oplus \ell} .
\end{gathered}
$$

Therefore we also have the following decomposition of functors:

$$
\begin{aligned}
& \mathrm{KZ}^{\circ}{ }^{\mathcal{O}} \operatorname{Res}_{n+m r} \circ{ }^{\mathcal{O}} \operatorname{Ind}_{n, m r} \\
& \quad=\left(\operatorname{KZ}{ }^{\mathcal{O}} \operatorname{Ind}_{n-1, m r} \circ\left({ }^{\mathcal{O}} \operatorname{Res}_{n} \otimes \mathbf{1}\right)\right) \oplus\left(\operatorname{KZ}{ }^{\mathcal{O}} \operatorname{Ind}_{n, m r-1} \circ\left(\mathbf{1} \otimes{ }^{\mathcal{O}} \operatorname{Res}_{m r}\right)\right)
\end{aligned}
$$

The induction and restriction functors on $\mathcal{O}(\Gamma)$ take projective modules to projective ones, because they are exact and biadjoint. Thus, by 3.7 we have a natural isomorphism

$$
\begin{aligned}
& { }^{\mathcal{O}} \operatorname{Res}_{n+m r}{ }^{\mathcal{O}} \operatorname{Ind}_{n, m r}(P) \\
& \quad={ }^{\mathcal{O}} \operatorname{Ind}_{n-1, m r}\left({ }^{\mathcal{O}} \operatorname{Res}_{n} \otimes \mathbf{1}\right)(P) \oplus\left({ }^{\mathcal{O}} \operatorname{Ind}_{n, m r-1}\left(\mathbf{1} \otimes{ }^{\mathcal{O}} \operatorname{Res}_{m r}\right)(P)\right)^{\oplus \ell}
\end{aligned}
$$

for any projective module $P \in \mathcal{O}(\Gamma)$. Since $\mathcal{O}(\Gamma)$ has enough projective objects, this yields an isomorphism of functors

$$
{ }^{\mathcal{O}} \operatorname{Res}_{n+m r}{ }^{\mathcal{O}} \operatorname{Ind}_{n, m r}={ }^{\mathcal{O}} \operatorname{Ind}_{n-1, m r}\left({ }^{\mathcal{O}} \operatorname{Res}_{n} \otimes \mathbf{1}\right) \oplus\left({ }^{\mathcal{O}} \operatorname{Ind}_{n, m r-1}\left(\mathbf{1} \otimes{ }^{\mathcal{O}} \operatorname{Res}_{m r}\right)\right){ }^{\oplus \ell} .
$$

In particular, the projection yields a morphism of functors

$$
{ }^{\mathcal{O}} \operatorname{Res}_{n+m r}{ }^{\mathcal{O}} \operatorname{Ind}_{n, m r} \rightarrow{ }^{\mathcal{O}} \operatorname{Ind}_{n-1, m r}\left({ }^{\mathcal{O}} \operatorname{Res}_{n} \otimes \mathbf{1}\right) .
$$

Applying this to the module $M \otimes{ }^{\mathcal{O}} \operatorname{Ind}_{\left(m^{r}\right)}(L)$ yields an $\mathfrak{S}_{r}$-equivariant surjective morphism

$$
\begin{aligned}
\Psi(M):{ }^{\mathcal{O}} \operatorname{Res}_{n+m r}{ }^{\mathcal{O}} \operatorname{Ind}_{n, m r}\left(M \otimes{ }^{\mathcal{O}} \operatorname{Ind}_{\left(m^{r}\right)}(L)\right) & \\
& \rightarrow{ }^{\mathcal{O}} \operatorname{Ind}_{n-1, m r}\left({ }^{\mathcal{O}} \operatorname{Res}_{n}(M) \otimes{ }^{\mathcal{O}} \operatorname{Ind}_{\left(m^{r}\right)}(L)\right) .
\end{aligned}
$$

Now, by (5.1), the left-hand side is equal to $E \circ\left(a^{*}\right)^{r}(M)$ and the right-hand side is equal to $\left(a^{*}\right)^{r} \circ E(M)$. So by Proposition 5.13 and the fact that the actions of $\mathfrak{H}$ and $\widehat{\mathfrak{s l}}_{m}$ on $\mathcal{F}_{m, \ell}^{(s)}$ commute with each other, we have

$$
\left[E \circ\left(a^{*}\right)^{r}(M)\right]=\left[\left(a^{*}\right)^{r} \circ E(M)\right] .
$$

Thus $\Psi(M)$ is indeed an isomorphism. So (5.26) is proved. 


\subsection{Primitive modules.}

Definition 5.17. A module $M \in \mathcal{O}(\Gamma)$ is primitive if $R a_{*}(M)=0$ and $E(M)=0$ (or, equivalently, if $R^{i} a_{*}(M)=e_{q}(M)=0$ for all $\left.q, i\right)$. Let $\operatorname{PI}(\mathcal{O}(\Gamma)$ ) be the set of isomorphism classes of primitive simple modules.

Proposition 5.18. For $L \in \operatorname{Irr}\left(\mathcal{O}\left(\Gamma_{n}\right)\right)$ the following are equivalent:

(a) $L \in \operatorname{PI}\left(\mathcal{O}\left(\Gamma_{n}\right)\right)$,

(b) $L \in \operatorname{Irr}\left(\mathcal{O}\left(\Gamma_{n}\right)\right)_{0,0}$,

(c) $\operatorname{dim}(L)<\infty$.

Proof. Assume that $L \in \operatorname{Irr}\left(\mathcal{O}\left(\Gamma_{n}\right)\right)$. The equivalence of $(b)$ and $(c)$ is Remark 3.13. Let us prove that $(a) \Rightarrow(b)$. Fix $l, j \geqslant 0$ such that $\operatorname{Supp}(L)=X_{l, j}$. Set $i=n-l-m j$. We first prove that $j=0$. Assume that $j>0$. Then we have

$$
\Gamma_{l,\left(m^{j}\right)}=\Gamma_{l,\left(m^{j-1}\right)} \times \mathfrak{S}_{m}, \quad \Gamma_{l,\left(m^{j-1}\right)} \subset \Gamma_{n-m} .
$$

There are modules $M_{\mu} \in \mathcal{O}\left(\Gamma_{n-m}\right), \mu \in \mathcal{P}_{m}$, such that in $\left[\mathcal{O}\left(\Gamma_{n, m}\right)\right]$ we have

$$
\left[\operatorname{Res}_{n, m}(L)\right]=\sum_{\mu \in \mathcal{P}_{m}}\left[M_{\mu} \otimes L_{\mu}\right]
$$

The transitivity of the restriction functor [33, cor. 2.5] yields the following formula:

$$
\left[\operatorname{Res}_{1}(L)\right]=\sum_{\mu}\left[\operatorname{Res}_{2}\left(M_{\mu}\right) \otimes L_{\mu}\right], \quad \operatorname{Res}_{1}={ }^{\mathcal{O}} \operatorname{Res}_{\Gamma_{l,(m j} \Gamma_{n}}^{\Gamma_{n}}, \quad \operatorname{Res}_{2}={ }^{\mathcal{O}} \operatorname{Res}_{\left.\Gamma_{l,(m} \Gamma^{n-1}\right)} \Gamma_{n-m} .
$$

The $H\left(\Gamma_{l,\left(m^{j}\right)}\right)$-module $\operatorname{Res}_{1}(L)$ is finite dimensional, because $\operatorname{Supp}(L)=X_{l, j}$. Thus we have $\operatorname{Res}_{2}\left(M_{\mu}\right)=0$ unless $\mu=(m)$, and

$$
\left[\operatorname{Res}_{1}(L)\right]=\left[\operatorname{Res}_{2}\left(M_{(m)}\right) \otimes L_{(m)}\right] .
$$

Next, since $R a_{*}([L])=0$ we have

$$
\begin{aligned}
0 & =\left[\operatorname{Res}_{2} \operatorname{Ra}_{*}(L)\right] \\
& =\sum_{\mu \in \mathcal{P}_{m}}\left[\operatorname{Res}_{2}\left(M_{\mu}\right) \otimes \operatorname{RHom}_{\mathcal{O}\left(\mathfrak{S}_{m}\right)}\left(L_{(m)}, L_{\mu}\right)\right] \\
& =\left[\operatorname{Res}_{2}\left(M_{(m)}\right) \otimes \operatorname{REnd}_{\mathcal{O}\left(\mathfrak{S}_{m}\right)}\left(L_{(m)}\right)\right] .
\end{aligned}
$$

Thus, using [3, thm. 1.3] we get $\operatorname{Res}_{2}\left(M_{(m)}\right)=0$. This yields a contradiction with (5.27) because $\operatorname{Res}_{1}(L) \neq 0$. So we have $j=0$. Next, since $E(L)=0$, by Corollary 3.19 and Remark 3.11 we have $i=0$.

Finally, we prove that $(c) \Rightarrow(a)$. We must prove that if $L$ is finite dimensional, then it is primitive. This is obvious, because ${ }^{\mathcal{O}} \operatorname{Res}_{n, m}(L)={ }^{\mathcal{O}} \operatorname{Res}_{n}(L)=0$.

Remark 5.19. By Proposition 5.18 the elements of $\operatorname{PI}\left(\mathcal{O}\left(\Gamma_{n}\right)\right)$ form a basis of $F_{0,0}\left(\Gamma_{n}\right)$.

5.5. Endomorphisms of induced modules. For $r \geqslant 1$ we consider the algebras

$$
B_{r}=\mathfrak{S}_{r} \ltimes \mathbb{C}\left[x_{1}, x_{2}, \ldots, x_{r}\right], \quad B_{r, \ell}=B_{r} /\left(x_{1}^{\ell}, x_{2}^{\ell}, \ldots, x_{r}^{\ell}\right) .
$$

The following proposition is the main result of this subsection.

Proposition 5.20. Let $r \geqslant 1$.

(a) The $\mathbb{C}$-algebra homomorphism $\mathbb{C S}_{r} \rightarrow \operatorname{End}_{\mathcal{O}(\Gamma)}\left(\left(a^{*}\right)^{r}\right)$ in Proposition 5.4 extends to a $\mathbb{C}$-algebra homomorphism $B_{r} \rightarrow \operatorname{End}_{\mathcal{O}(\Gamma)}\left(\left(a^{*}\right)^{r}\right)$ such that $x_{1}, x_{2}, \ldots, x_{r}$ map to nilpotent operators in $\operatorname{End}_{\mathcal{O}(\Gamma)}\left(\left(a^{*}\right)^{r}(L)\right)$ for each $L \in \mathcal{O}(\Gamma)$. 
(b) The $\mathbb{C}$-algebra homomorphism $B_{r} \rightarrow \operatorname{End}_{\mathcal{O}(\Gamma)}\left(\left(a^{*}\right)^{r}\right)$ factors to an isomorphism $B_{r, \ell}=\operatorname{End}_{\mathcal{O}(\Gamma)}\left(\left(a^{*}\right)^{r}(L)\right)$ for $L \in \mathrm{PI}(\mathcal{O}(\Gamma))$.

Proof. The proof of this proposition is done in several steps. Let $\mathbf{H}\left(\Gamma_{n,\left(m^{r}\right)}\right), \mathbf{H}\left(\Gamma_{n}\right)$ and $X_{i}$ be as in Appendix $\mathrm{A}$. Consider the elements

$$
\xi_{i}=X_{n+m(i-1)+1} X_{n+m(i-1)+2} \cdots X_{n+m i}, \quad i=1,2, \ldots, r .
$$

They belong to the centralizer of $\mathbf{H}\left(\Gamma_{n,\left(m^{r}\right)}\right)$ in $\mathbf{H}\left(\Gamma_{n+m r}\right)$. Thus the right multiplication by $\xi_{i}, i=1,2, \ldots, r$, defines an automorphism ${ }^{\mathbf{H}} \xi_{i}$ of the functor ${ }^{\mathbf{H}} \operatorname{Ind}_{n,\left(m^{r}\right)}$. More precisely, for an $\mathbf{H}\left(\Gamma_{n,\left(m^{r}\right)}\right)$-module $M$ we set

$$
\mathbf{H}_{\xi_{i}}(h \otimes v)=h \xi_{i} \otimes v, \quad h \in \mathbf{H}\left(\Gamma_{n+m r}\right), \quad v \in M .
$$

The functor $\mathrm{KZ}$ yields a $\mathbb{C}$-algebra isomorphism (5.3)

$$
\mathrm{KZ}: \operatorname{End}\left({ }^{\mathcal{O}} \operatorname{Ind}_{n,\left(m^{r}\right)}\right) \rightarrow \operatorname{End}\left({ }^{\mathbf{H}} \operatorname{Ind}_{n,\left(m^{r}\right)} \circ \mathrm{KZ}\right) .
$$

Thus there is a unique endomorphism ${ }^{\mathcal{O}} \xi_{i}$ of the functor ${ }^{\mathcal{O}} \operatorname{Ind}_{n,\left(m^{r}\right)}$ such that

$$
\mathrm{KZ}\left({ }^{\mathcal{O}} \xi_{i}(M)\right)={ }^{\mathbf{H}_{i}} \xi_{i}(\mathrm{KZ}(M)), \quad \forall M \in \mathcal{O}\left(\Gamma_{n,\left(m^{r}\right)}\right) .
$$

The functor $\bullet \otimes L: \mathcal{O}\left(\Gamma_{n}\right) \rightarrow \mathcal{O}\left(\Gamma_{n,\left(m^{r}\right)}\right)$ yields a $\mathbb{C}$-algebra homomorphism

$$
\operatorname{End}\left({ }^{\mathcal{O}} \operatorname{Ind}_{n,\left(m^{r}\right)}\right) \rightarrow \operatorname{End}\left(\left(a^{*}\right)^{r}\right) .
$$

Let $\bar{\xi}_{i}$ denote the image of $\mathcal{O}_{i}$ by the map (5.29). Next, recall the operators

$$
\bar{\tau}_{i} \in \operatorname{End}\left(\left(a^{*}\right)^{r}\right)=\operatorname{End}\left(\left(A^{*}\right)^{r}\right), \quad i=1,2, \ldots, r-1
$$

defined before Lemma 5.3

Lemma 5.21. The following relations hold in $\operatorname{End}\left(\left(a^{*}\right)^{r}\right)$ for $j \neq i, i+1$ :

$$
\bar{\tau}_{i} \circ \bar{\xi}_{i} \circ \bar{\tau}_{i}=\bar{\xi}_{i+1}, \quad \bar{\tau}_{i} \circ \bar{\xi}_{j} \circ \bar{\tau}_{i}=\bar{\xi}_{j} .
$$

Proof. Recall the permutation $\tau_{i}$ and the morphism of functors $\mathbf{H}_{\tau_{i}}$ defined in the beginning of Section 5.2. By (5.28) and (5.4) it is enough to prove that

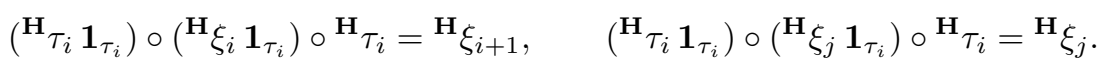

To do so, we are reduced to checking the following relations in $\mathbf{H}\left(\Gamma_{n+m r}\right)$ :

$$
T_{\tau_{i}} \xi_{i} T_{\tau_{i}}=\xi_{i+1}, \quad T_{\tau_{i}} \xi_{j} T_{\tau_{i}}=\xi_{j} .
$$

Recall that $\zeta$ is an $m$-th root of 1 . Let $a_{i}=n+(i-1) m+1, b_{i}=n+i m$, and

$$
K_{l}=T_{b_{i}-l} T_{b_{i}-l+2} \cdots T_{b_{i}+l-2} T_{b_{i}+l} .
$$

Since both words define the same element in the braid group, we have an equality

$$
T_{\tau_{i}}=K_{0} K_{1} \cdots K_{m-2} K_{m-1} K_{m-2} \cdots K_{1} K_{0} .
$$

Further, for $0 \leqslant l \leqslant m-1$ we have

$$
\begin{aligned}
& K_{l} X_{a_{i}} X_{a_{i}+1} \cdots X_{b_{i}-l-2} X_{b_{i}-l-1}\left(X_{b_{i}-l} X_{b_{i}-l+2} \cdots X_{b_{i}+l}\right) K_{l} \\
& \quad=\zeta^{l+1} X_{a_{i}} X_{a_{i}+1} \cdots X_{b_{i}-l-2}\left(X_{b_{i}-l-1} X_{b_{i}-l+1} X_{b_{i}-l+3} \cdots X_{b_{i}+l+1}\right),
\end{aligned}
$$

and for $0 \leqslant l \leqslant m-2$ we have

$$
\begin{aligned}
& K_{l}\left(X_{b_{i}-l} X_{b_{i}-l+2} \cdots X_{b_{i}+l}\right) X_{b_{i}+l+2} X_{b_{i}+l+3} \cdots X_{b_{i}+m} K_{l} \\
& \quad=\zeta^{l+1}\left(X_{b_{i}-l+1} X_{b_{i}-l+3} \cdots X_{b_{i}+l-1}\right) X_{b_{i}+l+1} X_{b_{i}+l+2} X_{b_{i}+l+3} \cdots X_{b_{i}+m} .
\end{aligned}
$$


We deduce that

$$
\begin{aligned}
T_{\tau_{i}} \xi_{i} T_{\tau_{i}} & =T_{\tau_{i}} X_{a_{i}} X_{a_{i}+1} \cdots X_{b_{i}} T_{\tau_{i}} \\
& =\zeta^{1+2+\cdots+m} K_{0} \cdots K_{m-2} X_{a_{i}+1} X_{a_{i}+3} \cdots X_{b_{i}+m-2} X_{b_{i}+m} K_{m-2} \cdots K_{0} \\
& =\zeta^{1+2+\cdots+m} \zeta^{1+2+\cdots+m-1} X_{a_{i}+m} X_{a_{i}+m+1} \cdots X_{b_{i}+m} \\
& =\zeta^{m^{2}} \xi_{i+1} \\
& =\xi_{i+1} .
\end{aligned}
$$

The relation $T_{\tau_{i}} \xi_{j} T_{\tau_{i}}=\xi_{j}$ for $j \neq i, i+1$ is obvious.

For any element $w \in \mathfrak{S}_{r}$ and any decomposition $w=s_{i_{1}} s_{i_{2}} \cdots s_{i_{k}}$, we set

$$
\bar{\tau}_{w}=\bar{\tau}_{i_{1}} \bar{\tau}_{i_{2}} \cdots \bar{\tau}_{i_{k}} \in \operatorname{End}\left(\left(a^{*}\right)^{r}\right) .
$$

This definition does not depend on the choice of the decomposition of $w$ by Lemma 5.3. Next, for a tuple $p=\left(p_{1}, p_{2}, \ldots, p_{r}\right) \in \mathbb{Z}^{r}$ such that $0 \leqslant p_{i}<\ell$ we set

$$
\xi^{p}=\xi_{1}^{p_{1}} \xi_{2}^{p_{2}} \cdots \xi_{r}^{p_{r}}, \quad \bar{\xi}^{p}=\bar{\xi}_{1}^{p_{1}} \bar{\xi}_{2}^{p_{2}} \cdots \bar{\xi}_{r}^{p_{r}} .
$$

Lemma 5.22. For any $L \in \operatorname{Irr}(\mathcal{O}(\Gamma))$ the elements $\bar{\xi}^{p} \bar{\tau}_{w}(L)$ of $\operatorname{End}_{\mathcal{O}(\Gamma)}\left(\left(a^{*}\right)^{r}(L)\right)$, with $w \in \mathfrak{S}_{r}$ and $p \in[0, \ell)^{r}$, are linearly independent.

Proof. If the decomposition $w=s_{i_{1}} s_{i_{2}} \cdots s_{i_{k}}$ is reduced, then the length of $\tau_{w}$ is the sum of the lengths of $\tau_{i_{1}}, \tau_{i_{2}}, \ldots, \tau_{i_{k}}$. For a tuple $p$ as above, we define the following elements in $\mathbf{H}\left(\Gamma_{n+m r}\right)$ :

$$
t_{w}=T_{\tau_{w}}, \quad \xi^{p}=\xi_{1}^{p_{1}} \xi_{2}^{p_{2}} \cdots \xi_{r}^{p_{r}} .
$$

Recall that the elements

$$
X_{1}^{p_{1}} X_{2}^{p_{2}} \cdots X_{n+m r}^{p_{n+m r}} T_{u}, \quad p_{i} \in[0, \ell), \quad u \in \mathfrak{S}_{n+m r},
$$

form a $\mathbb{C}$-basis of $\mathbf{H}\left(\Gamma_{n+m r}\right)$. Further $\xi^{p}$ centralizes $\mathbf{H}\left(\Gamma_{n,\left(m^{r}\right)}\right)$ and the element

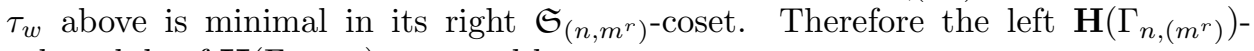
submodule of $\mathbf{H}\left(\Gamma_{n+m r}\right)$, spanned by

$$
\left\{\xi^{p} t_{w} ; w \in \mathfrak{S}_{r}, p \in[0, \ell)^{r}\right\},
$$

is indeed the direct sum

$$
\bigoplus_{p, w} \mathbf{H}\left(\Gamma_{n,\left(m^{r}\right)}\right) \xi^{p} t_{w}
$$

where $p$ runs over $[0, \ell)^{r}$ and $w$ over $\mathfrak{S}_{r}$. In other words, there is an injective $\mathbf{H}\left(\Gamma_{n,\left(m^{r}\right)}\right)$-module homomorphism

$$
\mathbf{H}_{\psi: \mathbf{H}\left(\Gamma_{n,\left(m^{r}\right)}\right)}^{\oplus \ell^{r} r !} \rightarrow \mathbf{H}\left(\Gamma_{n+m r}\right), \quad\left(h_{p, w}\right) \mapsto \sum_{p, w} h_{p, w} \xi^{p} t_{w},
$$

where $w, p$ run over $\mathfrak{S}_{r},[0, \ell)^{r}$ respectively. Further, since $\xi^{p}$ centralizes $\mathbf{H}\left(\Gamma_{n,\left(m^{r}\right)}\right)$, the relation (5.2) yields

$$
z \xi^{p} t_{w}=\xi^{p} z t_{w}=\xi^{p} t_{w} \tau_{w^{-1}}(z), \quad z \in \mathbf{H}\left(\Gamma_{n,\left(m^{r}\right)}\right) .
$$

Therefore ${ }^{\mathbf{H}} \psi$ is a $\left(\mathbf{H}\left(\Gamma_{n,\left(m^{r}\right)}\right), \mathbf{H}\left(\Gamma_{n,\left(m^{r}\right)}\right)\right)$-bimodule homomorphism, where the right $\mathbf{H}\left(\Gamma_{n,\left(m^{r}\right)}\right)$-action on $\mathbf{H}\left(\Gamma_{n,\left(m^{r}\right)}\right)^{\oplus \ell^{r} r !}$ is twisted in the obvious way. Since $\mathbf{H}_{\psi}$ 
is injective, and both sides are free $\mathbf{H}\left(\Gamma_{n,\left(m^{r}\right)}\right)$-modules, for each $M \in \mathcal{O}\left(\Gamma_{n,\left(m^{r}\right)}\right)$ we have an injective homomorphism

$$
\begin{aligned}
{ }^{\mathbf{H}} \psi(\mathrm{KZ}(M)): & \bigoplus_{p, w} \tau_{w} \mathrm{KZ}(M) \rightarrow{ }^{\mathbf{H}} \operatorname{Res}_{n,\left(m^{r}\right)} \circ{ }^{\mathbf{H}} \operatorname{Ind}_{n,\left(m^{r}\right)} \mathrm{KZ}(M) \\
& =\mathrm{KZ}^{\circ}{ }^{\mathcal{O}} \operatorname{Res}_{n,\left(m^{r}\right)} \circ{ }^{\mathcal{O}} \operatorname{Ind}_{n,\left(m^{r}\right)}(M),
\end{aligned}
$$

where $\tau_{w}: \operatorname{Rep}\left(\mathbf{H}\left(\Gamma_{n,\left(m^{r}\right)}\right)\right) \rightarrow \operatorname{Rep}\left(\mathbf{H}\left(\Gamma_{n,\left(m^{r}\right)}\right)\right)$. Further, we have

$$
\tau_{w} \circ \mathrm{KZ}=\mathrm{KZ} \circ \tau_{w},
$$

where $\tau_{w}: \mathcal{O}\left(\Gamma_{n,\left(m^{r}\right)}\right) \rightarrow \mathcal{O}\left(\Gamma_{n,\left(m^{r}\right)}\right)$ is the twist by the permutation

$$
H\left(\Gamma_{n,\left(m^{r}\right)}\right)=H\left(\Gamma_{n}\right) \otimes H\left(\mathfrak{S}_{m}\right)^{\otimes r} \rightarrow H\left(\Gamma_{n}\right) \otimes H\left(\mathfrak{S}_{m}\right)^{\otimes r}=H\left(\Gamma_{n,\left(m^{r}\right)}\right) .
$$

Let $S: \operatorname{Rep}(\mathbf{H}(\Gamma)) \rightarrow \mathcal{O}(\Gamma)$ be as in Section 3.7. The canonical adjunction morphism $P \rightarrow S(\mathrm{KZ}(P))$ is an isomorphism for each projective module $P \in \mathcal{O}(\Gamma)$. Further, the functors ${ }^{\mathcal{O}} \operatorname{Res}_{n,\left(m^{r}\right)}$ and ${ }^{\mathcal{O}} \operatorname{Ind}_{n,\left(m^{r}\right)}$ preserve the projective objects, because they are bi-adjoint and exact. Therefore, applying the left exact functor $S$ to the map ${ }^{\mathbf{H}} \psi(\mathrm{KZ}(P))$, with $P$ projective in $\mathcal{O}\left(\Gamma_{n,\left(m^{r}\right)}\right)$, we get an injection

$$
{ }^{\mathcal{O}} \psi(P): \bigoplus_{w} \tau_{w}(P)^{\oplus \ell^{r}} \rightarrow{ }^{\mathcal{O}} \operatorname{Res}_{n,\left(m^{r}\right)}{ }^{\mathcal{O}} \operatorname{Ind}_{n,\left(m^{r}\right)}(P) .
$$

Since the category $\mathcal{O}\left(\Gamma_{n,\left(m^{r}\right)}\right)$ has enough projective objects and since the functor ${ }^{\mathcal{O}} \operatorname{Res}_{n,\left(m^{r}\right)}{ }^{\mathcal{O}} \operatorname{Ind}_{n,\left(m^{r}\right)}$ is exact, the five lemma implies that there is a functorial injective morphism

$$
{ }^{\mathcal{O}} \psi(M): \bigoplus_{w} \tau_{w}(M)^{\oplus \ell^{r}} \rightarrow{ }^{\mathcal{O}} \operatorname{Res}_{n,\left(m^{r}\right)}{ }^{\mathcal{O}} \operatorname{Ind}_{n,\left(m^{r}\right)}(M), \quad M \in \mathcal{O}\left(\Gamma_{n,\left(m^{r}\right)}\right) .
$$

Now, set $M=L \otimes L_{(m)}^{\otimes r}$ with $L \in \operatorname{Irr}(\mathcal{O}(\Gamma))$. Then we have $\tau_{w}(M)=M$ for all $w$ as above. Therefore we get an injective linear map

$$
\begin{aligned}
& \mathbb{C}^{\ell^{r} r !}=\operatorname{Hom}_{\mathcal{O}(\Gamma)}\left(L \otimes L_{(m)}^{\otimes r}, L \otimes L_{(m)}^{\otimes r}\right) \oplus \ell^{r} r ! \\
& \rightarrow \operatorname{Hom}_{\mathcal{O}(\Gamma)}\left(L \otimes L_{(m)}^{\otimes r},{ }^{\mathcal{O}} \operatorname{Res}_{n,\left(m^{r}\right)}{ }^{\mathcal{O}} \operatorname{Ind}_{n,\left(m^{r}\right)}\left(L \otimes L_{(m)}^{\otimes r}\right)\right)=\operatorname{End}_{\mathcal{O}(\Gamma)}\left(\left(a^{*}\right)^{r}(L)\right) .
\end{aligned}
$$

It maps the canonical basis elements to the elements $\bar{\xi}^{p} \bar{\tau}_{w}(L)$ with $w \in \mathfrak{S}_{r}$ and $p \in[0, \ell)^{r}$.

Lemma 5.23. For $L \in \operatorname{PI}\left(\mathcal{O}\left(\Gamma_{n}\right)\right)$ the following identity holds in $\left[\mathcal{O}\left(\Gamma_{n,\left(m^{r}\right)}\right)\right]$ :

$$
\left[{ }^{\mathcal{O}} \operatorname{Res}_{n,\left(m^{r}\right)}\left(a^{*}\right)^{r}(L)\right]=\ell^{r} r !\left[L \otimes L_{(m)}^{\otimes r}\right] .
$$

Proof. By Lemma 2.5 the left-hand side is equal to

$$
\sum_{x}{ }^{\mathcal{O}} \operatorname{Ind}_{x^{-1} W_{x} x}^{\Gamma_{n,\left(m^{r}\right)}} \circ^{x}\left({ }^{\mathcal{O}} \operatorname{Res}_{W_{x}}^{\Gamma_{n,\left(m^{r}\right)}}\left(\left[L \otimes L_{(m)}^{\otimes r}\right]\right)\right),
$$

where $W_{x}=x \Gamma_{n,\left(m^{r}\right)} x^{-1} \cap \Gamma_{n,\left(m^{r}\right)}$ and $x$ runs over a set of representatives of the double cosets in $\Gamma_{n,\left(m^{r}\right)} \backslash \Gamma_{n+m r} / \Gamma_{n,\left(m^{r}\right)}$. Since $W_{x}$ is a parabolic subgroup of $\Gamma_{n,\left(m^{r}\right)}$, it is generated by reflections. Hence we can decompose the group $W_{x}$ in the following way:

$$
W_{x}=W_{x}^{\prime} \times W_{x}^{\prime \prime}, \quad W_{x}^{\prime} \subset \Gamma_{n}, \quad W_{x}^{\prime \prime} \subset \mathfrak{S}_{m}^{r} .
$$

Here $W_{x}^{\prime}, W_{x}^{\prime \prime}$ are parabolic subgroups. We have

$$
{ }^{\mathcal{O}} \operatorname{Res}_{W_{x},\left(m^{r}\right)}^{\Gamma_{n}}\left(L \otimes L_{(m)}^{\otimes r}\right)={ }^{\mathcal{O}} \operatorname{Res}_{W_{x}^{\prime}}^{\Gamma_{n}}(L) \otimes{ }^{\mathcal{O}} \operatorname{Res}_{W_{x}^{\prime \prime}}^{\mathfrak{S}_{m}^{r}}\left(L_{(m)}^{\otimes r}\right),
$$


and a similar decomposition holds for the induction functor. Further, since $L \in$ $\operatorname{PI}\left(\mathcal{O}\left(\Gamma_{n}\right)\right)$ we have ${ }^{\mathcal{O}} \operatorname{Res}_{W^{\prime}}^{\Gamma_{n}}(L)=0$ if $W_{x}^{\prime}$ is proper by Proposition 5.18. Thus we can assume that $W_{x}^{\prime}=\Gamma_{n}$; i.e., we can assume that $x$ belongs to the subgroup $\{1\} \times \Gamma_{m r} \subset \Gamma_{n+m r}$. We'll abbreviate

$$
\mathfrak{S}_{m}^{r}=\{1\} \times \mathfrak{S}_{m}^{r}, \quad \Gamma_{m r}=\{1\} \times \Gamma_{m r} .
$$

Then we have $W_{x}^{\prime \prime}=x \mathfrak{S}_{m}^{r} x^{-1} \cap \mathfrak{S}_{m}^{r}$, and we are reduced to checking that

$$
\sum_{x}{ }^{\mathcal{O}} \operatorname{Ind}_{x^{-1} W_{x} x}^{\mathfrak{S}_{m}^{r}} \circ{ }^{x}\left({ }^{\mathcal{O}} \operatorname{Res}_{W_{x}}^{\mathfrak{S}_{m}^{r}}\left(\left[L_{(m)}^{\otimes r}\right]\right)\right)=\ell^{r} r !\left[L_{(m)}^{\otimes r}\right]
$$

where $W_{x}=x \mathfrak{S}_{m}^{r} x^{-1} \cap \mathfrak{S}_{m}^{r}$ and $x$ runs over a set of representatives of the double cosets in $\mathfrak{S}_{m}^{r} \backslash \Gamma_{m r} / \mathfrak{S}_{m}^{r}$. Now, observe that

$$
\mathcal{O}^{\mathcal{R e s}}{\stackrel{\mathfrak{S}}{W_{x}}}_{r}^{r}\left(L_{(m)}^{\otimes r}\right)=0
$$

unless $x \mathfrak{S}_{m}^{r} x^{-1}=\mathfrak{S}_{m}^{r}$, and that $x \mathfrak{S}_{m}^{r} x^{-1}=\mathfrak{S}_{m}^{r}$ if and only if $x$ belongs to $N_{\Gamma_{m r}}\left(\mathfrak{S}_{m}^{r}\right)$, the normalizer of $\mathfrak{S}_{m}^{r}$ in $\Gamma_{m r}$. Further, we have a group isomorphism

$$
N_{\Gamma_{m r}}\left(\mathfrak{S}_{m}^{r}\right) / \mathfrak{S}_{m}^{r}=\Gamma_{r} .
$$

This proves the lemma.

Lemma 5.24. For $L \in \operatorname{PI}(\mathcal{O}(\Gamma))$ the elements $\bar{\xi}^{p} \bar{\tau}_{w}(L)$ with $w \in \mathfrak{S}_{r}$ and $p \in[0, \ell)^{r}$ form a basis of $\operatorname{End}_{\mathcal{O}(\Gamma)}\left(\left(a^{*}\right)^{r}(L)\right)$.

Proof. By Lemma 5.22 it is enough to check that

$$
\operatorname{dim} \operatorname{End}_{\mathcal{O}(\Gamma)}\left(\left(a^{*}\right)^{r}(L)\right) \leqslant \ell^{r} r ! .
$$

For $L \in \operatorname{PI}\left(\mathcal{O}\left(\Gamma_{n}\right)\right)$ Lemma 5.23 yields

$$
\operatorname{dim} \operatorname{End}_{\mathcal{O}(\Gamma)}\left(\left(a^{*}\right)^{r}(L)\right)=\operatorname{dim}_{\operatorname{Hom}_{\mathcal{O}(\Gamma)}}\left(L \otimes L_{(m)}^{\otimes r},{ }^{\mathcal{O}} \operatorname{Res}_{n,\left(m^{r}\right)}\left(a^{*}\right)^{r}(L)\right) \leqslant \ell^{r} r ! .
$$

Lemma 5.25. For $i=1,2, \ldots, r$ and $L \in \mathcal{O}(\Gamma)$ the operator $\bar{\xi}_{i}(L)+1$ on $\left(a^{*}\right)^{r}(L)$ is nilpotent. Further, if $L \in \operatorname{PI}(\mathcal{O}(\Gamma))$ we have $\left(\bar{\xi}_{i}(L)+1\right)^{\ell}=0$.

Proof. The $\mathbb{C}$-vector space $[\mathcal{O}(\Gamma)]$ is equipped with an $\widetilde{\mathfrak{s l}}_{m}$-action via the isomorphism (5.20); see also Remark 4.7. For a weight $\mu$ of $\widetilde{\mathfrak{s l}}_{m}$ let $\mathcal{O}(\Gamma)_{\mu} \subset \mathcal{O}(\Gamma)$ be the Serre subcategory generated by the simple modules $L$ whose class in $[\mathcal{O}(\Gamma)]$ has the weight $\mu$. Set $\mathcal{O}\left(\Gamma_{n}\right)_{\mu}=\mathcal{O}(\Gamma)_{\mu} \cap \mathcal{O}\left(\Gamma_{n}\right)$. Although we'll not need this formula, note that if $\Delta_{\lambda} \in \mathcal{O}\left(\Gamma_{n}\right)_{\mu}$, then we have

$$
\mu=\mu_{0}-\sum_{q=0}^{m-1} n_{q}(\lambda) \alpha_{q},
$$

where $\mu_{0}$ is a weight which does not depend on $n, \lambda$, and $n_{q}(\lambda)$ is the number of $q$-nodes in the $\ell$-partition $\lambda$. The element

$$
z_{n}=X_{1} X_{2} \cdots X_{n}
$$

belongs to the center of $\mathbf{H}\left(\Gamma_{n}\right)$. Thus it yields an element $\mathbf{H}_{z_{n}}$ in the center of $\operatorname{Rep}\left(\mathbf{H}\left(\Gamma_{n}\right)\right)$. Since KZ identifies the centers of $\mathcal{O}\left(\Gamma_{n}\right)$ and $\operatorname{Rep}\left(\mathbf{H}\left(\Gamma_{n}\right)\right)$, it yields also an element ${ }^{\mathcal{O}} z_{n}$ in the center of $\mathcal{O}\left(\Gamma_{n}\right)$. Let $L \in \operatorname{Irr}\left(\mathcal{O}\left(\Gamma_{n}\right)_{\mu}\right)$. Then ${ }^{\mathcal{O}} z_{n}$ acts on $L$ by multiplication by the scalar $\zeta^{\nu(\mu)}$, where $\nu$ is a linear form such that $\nu\left(\alpha_{i}\right)=i$ for $i=0,1, \ldots, m-1$; see, e.g., [33, sec. 4.1]. Now the operator $a^{*}$ maps $\mathcal{O}\left(\Gamma_{n}\right)_{\mu}$ 
to $\mathcal{O}\left(\Gamma_{n+m}\right)_{\mu+\delta}$ by Proposition 5.13 . Thus ${ }^{\mathcal{O}} z_{n+m}$ acts on $a^{*}(L)$ by multiplication by the scalar $\zeta^{\nu(\mu+\delta)}$. Therefore $\bar{\xi}_{1}$ acts on $a^{*}(L)$ by multiplication by the scalar

$$
\zeta^{\nu(\delta)}=\zeta^{m(m-1) / 2}=-1 .
$$

By Lemmas 5.21, 5.3, this implies that for any $L \in \mathcal{O}(\Gamma)$ we have $\left(\bar{\xi}_{i}(L)+1\right)^{N}=0$ in $\operatorname{End}_{\mathcal{O}(\Gamma)}\left(\left(a^{*}\right)^{r}(L)\right)$ for $i=1,2, \ldots, r$ and $N$ large enough.

Now, assume that $L \in \operatorname{PI}(\mathcal{O}(\Gamma))$. Let $N_{i}$ be the minimal integer such that $\left(\bar{\xi}_{i}(L)+1\right)^{N_{i}}=0$. By Lemmas 5.21, 5.3 we have $N_{1}=N_{2}=\cdots=N_{r}$. Hence, by Lemma 5.22 we also have $\ell=N_{1}=N_{2}=\cdots=N_{r}$.

Now we complete the proof of Proposition 5.20. The previous lemmas imply that the assignment

$$
x_{i} \mapsto \bar{\xi}_{i}+1, \quad s_{j} \mapsto \bar{\tau}_{j}, \quad i=1,2, \ldots, r, \quad j=1,2, \ldots, r-1,
$$

yields a $\mathbb{C}$-algebra morphism $B_{r} \rightarrow \operatorname{End}_{\mathcal{O}(\Gamma)}\left(\left(a^{*}\right)^{r}\right)$ such that $x_{i}$ maps to a nilpotent operator in $\operatorname{End}_{\mathcal{O}(\Gamma)}\left(\left(a^{*}\right)^{r}(L)\right)$ for each $L \in \mathcal{O}(\Gamma)$. The action of $s_{j}$ on $\left(a^{*}\right)^{r}$ given above is the same as the action of $s_{j}$ on $\left(A^{*}\right)^{r}$ in Proposition 5.4. This proves part (a). Part (b) follows from Lemmas 5.24, 5.25.

For a module $M$ in $\mathcal{O}(\Gamma)$ the adjunction yields a morphism

$$
\eta(M): M \otimes L_{(m)}^{\otimes r} \rightarrow{ }^{\mathcal{O}} \operatorname{Res}_{n,\left(m^{r}\right)}\left(a^{*}\right)^{r}(M) .
$$

Corollary 5.26. For $r \geqslant 1$ and $L \in \operatorname{PI}\left(\mathcal{O}\left(\Gamma_{n}\right)\right)$ the $\mathbb{C}$-algebra isomorphism (5.32)

$$
B_{r, \ell}=\operatorname{End}_{\mathcal{O}(\Gamma)}\left(\left(a^{*}\right)^{r}(L)\right)
$$

yields an isomorphism of $B_{r, \ell} \times H\left(\Gamma_{n,\left(m^{r}\right)}\right)$-modules

$$
B_{r, \ell} \otimes\left(L \otimes L_{(m)}^{\otimes r}\right) \rightarrow{ }^{\mathcal{O}} \operatorname{Res}_{n,\left(m^{r}\right)}\left(a^{*}\right)^{r}(L), \quad w \otimes v \mapsto{ }^{\mathcal{O}} \operatorname{Res}_{n,\left(m^{r}\right)}(w) \cdot \eta(L)(v) .
$$

Proof. The corollary follows from Proposition 5.20 and Lemma 5.23, because

$$
\operatorname{End}_{\mathcal{O}(\Gamma)}\left(\left(a^{*}\right)^{r}(L)\right)=\operatorname{Hom}_{\mathcal{O}(\Gamma)}\left(L \otimes L_{(m)}^{\otimes r},{ }^{\mathcal{O}} \operatorname{Res}_{n,\left(m^{r}\right)}\left(a^{*}\right)^{r}(L)\right)
$$

is a free $B_{r, \ell}$-module of rank one and, in $\left[\mathcal{O}\left(\Gamma_{n,\left(m^{r}\right)}\right)\right]$, we have

$$
\left[{ }^{\mathcal{O}} \operatorname{Res}_{n,\left(m^{r}\right)}\left(a^{*}\right)^{r}(L)\right]=\operatorname{dim}\left(B_{r, \ell}\right)\left[L \otimes L_{(m)}^{\otimes r}\right] .
$$

Definition 5.27. For $\lambda \in \mathcal{P}_{r}, r \geqslant 1$, we can regard the $\mathfrak{S}_{r}$-module $\bar{L}_{\lambda}$ as a $B_{r, \ell^{-}}$ module such that $x_{1}, x_{2}, \ldots, x_{r}$ act by zero. For $L \in \operatorname{PI}\left(\mathcal{O}\left(\Gamma_{n}\right)\right)$ we define

$$
\bar{a}_{\lambda}^{*}(L)=\bar{L}_{\lambda} \otimes_{B_{r, \ell}}\left(a^{*}\right)^{r}(L) \in \mathcal{O}\left(\Gamma_{n+m r}\right) .
$$

Definition 5.28. For $r \geqslant 1$ we define a functor $\mathcal{O}\left(\Gamma_{n+m r}\right) \rightarrow \operatorname{Rep}\left(\mathfrak{S}_{r}\right) \otimes \mathcal{O}\left(\Gamma_{n}\right)$ by

$$
\begin{aligned}
\Psi(M) & =\operatorname{Hom}_{\mathcal{O}\left(\mathfrak{S}_{m}^{r}\right)}\left(L_{(m)}^{\otimes r},{ }^{\mathcal{O}} \operatorname{Res}_{n,\left(m^{r}\right)}(M)\right) \\
& =\operatorname{Hom}_{\mathcal{O}\left(\mathfrak{S}_{m r}\right)}\left({ }^{\mathcal{O}} \operatorname{Ind}_{\left(m^{r}\right)}\left(L_{(m)}^{\otimes r}\right),{ }^{\mathcal{O}} \operatorname{Res}_{n, m r}(M)\right) .
\end{aligned}
$$

The $\mathfrak{S}_{r}$-action on $\Psi(M)$ is the $\mathfrak{S}_{r}$-action on ${ }^{\mathcal{O}} \operatorname{Ind}_{\left(m^{r}\right)}\left(L_{(m)}^{\otimes r}\right)$ in the proof of Proposition 5.4. In other words, we have $\Psi=\left(a_{*}\right)^{r}$, viewed as a $\mathfrak{S}_{r}$-equivariant functor as in the proof of Proposition 5.4. 
Corollary 5.29. For $r \geqslant 1$ and $L \in \operatorname{PI}\left(\mathcal{O}\left(\Gamma_{n}\right)\right)$ we have an isomorphism

$$
\left(L \otimes L_{(m)}^{\otimes r}\right) \oplus \operatorname{dim}\left(\bar{L}_{\lambda}\right)={ }^{\mathcal{O}} \operatorname{Res}_{n,\left(m^{r}\right)}\left(\bar{a}_{\lambda}^{*}(L)\right)
$$

as $H\left(\Gamma_{n,\left(m^{r}\right)}\right)$-modules, and we have an isomorphism of $\mathfrak{S}_{r} \times H\left(\Gamma_{n}\right)$-modules

$$
\bar{L}_{\lambda} \otimes L=\Psi\left(\bar{a}_{\lambda}^{*}(L)\right) .
$$

Proof. Corollary 5.26 yields an isomorphism

$$
B_{r, \ell} \otimes\left(L \otimes L_{(m)}^{\otimes r}\right)={ }^{\mathcal{O}} \operatorname{Res}_{n,\left(m^{r}\right)}\left(\left(a^{*}\right)^{r}(L)\right)
$$

which factors to an isomorphism

$$
\mathbb{C S}_{r} \otimes\left(L \otimes L_{(m)}^{\otimes r}\right)={ }^{\mathcal{O}} \operatorname{Res}_{n,\left(m^{r}\right)}\left(\overline{\left(a^{*}\right)^{r}}(L)\right),
$$

with

$$
\overline{\left(a^{*}\right)^{r}}(L)=\left(a^{*}\right)^{r}(L) / \sum_{i} x_{i}\left(a^{*}\right)^{r}(L) .
$$

Further, by taking the isotypic components we see that the isomorphism (5.33) factors to an isomorphism

$$
\left(L \otimes L_{(m)}^{\otimes r}\right){ }^{\oplus \operatorname{dim}\left(\bar{L}_{\lambda}\right)}={ }^{\mathcal{O}} \operatorname{Res}_{n,\left(m^{r}\right)}\left(\bar{a}_{\lambda}^{*}(L)\right) .
$$

This proves the first claim. To prove the second claim, observe that Corollary 5.26 and (5.33) yield compatible $\mathfrak{S}_{r} \times \mathfrak{S}_{r} \times H\left(\Gamma_{n}\right)$-module isomorphisms

$$
B_{r, \ell} \otimes L=\Psi\left(\left(a^{*}\right)^{r}(L)\right), \quad \mathbb{C S}_{r} \otimes L=\Psi\left(\overline{\left(a^{*}\right)^{r}}(L)\right) .
$$

The first $\mathfrak{S}_{r}$-action on $\Psi\left(\overline{\left(a^{*}\right)^{r}}(L)\right)$ is the $\mathfrak{S}_{r}$-action in the definition of $\Psi$, and the first $\mathfrak{S}_{r}$-action on $\mathbb{C S}_{r} \otimes L$ is the contragredient of the right $\mathfrak{S}_{r}$-action on $\mathbb{C S}_{r}$, i.e., the action such that $w \in \mathfrak{S}_{r}$ takes the element $a \otimes b$ in $\mathbb{C S}_{r} \otimes L$ to $a w^{-1} \otimes b$. The second $\mathfrak{S}_{r}$-action on $\Psi\left(\overline{\left(a^{*}\right)^{r}}(L)\right)$ is the $\mathfrak{S}_{r}$-action on $\overline{\left(a^{*}\right)^{r}}(L)$ in Corollary [5.26, and the second $\mathfrak{S}_{r}$-action on $\mathbb{C S}_{r} \otimes L$ is the left $\mathfrak{S}_{r}$-action on $\mathbb{C S}_{r}$. To identify the actions as above, it is enough to note that the isomorphism

$$
\begin{gathered}
B_{r, \ell}=\operatorname{Hom}_{\mathcal{O}\left(\Gamma_{n}\right)}\left(L, B_{r, \ell} \otimes L\right)=\operatorname{Hom}_{\mathcal{O}\left(\Gamma_{n}\right)}\left(L, \Psi\left(a^{*}\right)^{r}(L)\right) \\
=\operatorname{End}_{\mathcal{O}(\Gamma)}\left(\left(a^{*}\right)^{r}(L)\right)
\end{gathered}
$$

given by (5.34) is equal to the isomorphism (5.32), and that the $\mathfrak{S}_{r}$-actions on $\left(a^{*}\right)^{r}(L)$ are taken to the left and to the dual right $\mathfrak{S}_{r}$-action on $B_{r, \ell}$ by the map (5.35). Next, write

$$
\mathbb{C S}_{r}=\bigoplus_{\lambda} \bar{L}_{\lambda} \otimes \bar{L}_{\lambda}
$$

as an $\mathfrak{S}_{r} \times \mathfrak{S}_{r}$-module, and take the isotypic component.

\subsection{Definition of the map $\tilde{a}_{\lambda}$.}

Proposition 5.30. For $\lambda \in \mathcal{P}_{r}$ with $r \geqslant 1$ we have

$$
a_{\lambda}^{*}\left(F_{i, j}\left(\Gamma_{n}\right)\right) \subset F_{i, j+r}\left(\Gamma_{n+m r}\right), \quad a_{\lambda}^{*}\left(F_{i, j}\left(\Gamma_{n}\right)^{\circ}\right) \subset F_{i, j+r}\left(\Gamma_{n+m r}\right)^{\circ} .
$$

Proof. By Remark 3.15 we have

$$
\operatorname{Supp}\left(L_{m \lambda}\right)=X_{\mathfrak{S}_{m}^{r}, \mathbb{C}_{0}^{m r}}
$$

Let $L \in \operatorname{Irr}\left(\mathcal{O}\left(\Gamma_{n}\right)\right)$. First, assume that $L \in \operatorname{Irr}\left(\mathcal{O}\left(\Gamma_{n}\right)\right)_{i, j}$, i.e., that

$$
\operatorname{Supp}(L)=X_{l, j, \mathbb{C}^{n}}
$$


by Remark 3.12, Hence the module $L \otimes L_{m \lambda}$ has the following support:

$$
\operatorname{Supp}\left(L \otimes L_{m \lambda}\right)=X_{l, j, \mathbb{C}^{n}} \times X_{\mathfrak{S}_{m}^{r}, \mathbb{C}_{0}^{m r}}
$$

So by Proposition 2.7 we have

$$
\operatorname{Supp}\left(a_{\lambda}^{*}(L)\right)=X_{l, j+r, \mathbb{C}^{n+m r}} .
$$

Thus the class of $a_{\lambda}^{*}(L)$ belongs to $F_{i, j+r}\left(\Gamma_{n+m r}\right)^{\circ}$ by Remark 3.12. Next, assume that $[L] \in F_{i, j}\left(\Gamma_{n}\right)$, i.e.,

$$
\operatorname{Supp}(L)=X_{l^{\prime}, j^{\prime}, \mathbb{C}^{n}}, \quad X_{l^{\prime}, j^{\prime}, \mathbb{C}^{n}} \subset X_{l, j, \mathbb{C}^{n}}
$$

Thus we have

So (3.11) yields

$$
\operatorname{Supp}\left(a_{\lambda}^{*}\left(L^{\prime}\right)\right)=X_{l^{\prime}, j^{\prime}+r, \mathbb{C}^{n+m r}}
$$

$$
X_{l^{\prime}, j^{\prime}+r, \mathbb{C}^{n+m r}} \subset X_{l, j+r, \mathbb{C}^{n+m r}} ;
$$

i.e., the class of $a_{\lambda}^{*}(L)$ lies in $F_{i, j+r}\left(\Gamma_{n+m r}\right)$.

Proposition 5.31. Let $\lambda \in \mathcal{P}_{r}$ with $r \geqslant 1$, and let $L \in \operatorname{PI}\left(\mathcal{O}\left(\Gamma_{n}\right)\right)$. The module $\operatorname{top}\left(\bar{a}_{\lambda}^{*}(L)\right)$ has a unique constituent in $\operatorname{Irr}\left(\mathcal{O}\left(\Gamma_{n+m r}\right)\right)_{0, r}$.

Proof. Since the module $L$ is primitive, it belongs to $\operatorname{Irr}\left(\mathcal{O}\left(\Gamma_{n}\right)\right)_{0,0}$ by Proposition 5.18. Thus $\left[a_{\lambda}^{*}(L)\right] \in F_{0, r}\left(\Gamma_{n+m r}\right)$ by Proposition 5.30. Thus the constituents of $\bar{a}_{\lambda}^{*}(L)$ belong to the set

$$
\bigcup_{j \leqslant r} \operatorname{Irr}\left(\mathcal{O}\left(\Gamma_{n+m r}\right)\right)_{0, j}
$$

by Remark 3.11. Now, for $L^{\prime}$ in $\operatorname{Irr}\left(\mathcal{O}\left(\Gamma_{n+m r}\right)\right)_{0, j}$ we have ${ }^{\mathcal{O}} \operatorname{Res}_{n,\left(m^{r}\right)}\left(L^{\prime}\right)=0$ if $j<r$, and $\operatorname{dim}^{\mathcal{O}} \operatorname{Res}_{n,\left(m^{r}\right)}\left(L^{\prime}\right)<\infty$ if $j=r$. Further, the constituents of a finitedimensional module in $\mathcal{O}\left(\mathfrak{S}_{m}^{r}\right)$ are all isomorphic to $L_{(m)}^{\otimes r}$, and, using [3, thm. 1.3] as in the proof of Proposition [5.7, we get

$$
\operatorname{Ext}_{\mathcal{O}\left(\mathfrak{S}_{m}^{r}\right)}^{1}\left(L_{(m)}^{\otimes r}, L_{(m)}^{\otimes r}\right)=0 .
$$

Thus if $L^{\prime}$ is a constituent of $\operatorname{top}\left(\bar{a}_{\lambda}^{*}(L)\right)$, then we have a surjective map

$$
\Psi\left(\bar{a}_{\lambda}^{*}(L)\right) \rightarrow \Psi\left(L^{\prime}\right) .
$$

We also have

$$
\Psi\left(L^{\prime}\right)=\bigoplus_{\mu \in \mathcal{P}_{r}} \bar{L}_{\mu} \otimes \operatorname{Hom}_{\mathcal{O}\left(\mathfrak{S}_{m r}\right)}\left(L_{m \mu},{ }^{\mathcal{O}} \operatorname{Res}_{n, m r}\left(L^{\prime}\right)\right) .
$$

Finally, Corollary 5.29 yields an isomorphism of $\mathfrak{S}_{r} \otimes H\left(\Gamma_{n}\right)$-modules

$$
\bar{L}_{\lambda} \otimes L=\Psi\left(\bar{a}_{\lambda}^{*}(L)\right) .
$$

Thus the surjectivity of (5.36) implies that

$$
\operatorname{Hom}_{\mathcal{O}\left(\mathfrak{S}_{m r}\right)}\left(L_{m \mu},{ }^{\mathcal{O}} \operatorname{Res}_{n, m r}\left(L^{\prime}\right)\right)=0, \quad \forall \mu \neq \lambda .
$$

Since the $\mathfrak{S}_{r} \otimes H\left(\Gamma_{n}\right)$-module $\bar{L}_{\lambda} \otimes L$ is simple, the map (5.36) is invertible if it is nonzero. Assume further that $L^{\prime} \in \operatorname{Irr}\left(\mathcal{O}\left(\Gamma_{n+m r}\right)\right)_{0, r}$. Then Proposition 2.2 yields

$$
{ }^{\mathcal{O}} \operatorname{Res}_{n,\left(m^{r}\right)}\left(L^{\prime}\right) \neq 0 \text {. }
$$

Since $\operatorname{dim}^{\mathcal{O}} \operatorname{Res}_{n,\left(m^{r}\right)}\left(L^{\prime}\right)<\infty$ and the constituents of a finite-dimensional module in $\mathcal{O}\left(\mathfrak{S}_{m}^{r}\right)$ are all isomorphic to $L_{(m)}^{\otimes r}$, we also have $\Psi\left(L^{\prime}\right) \neq 0$. Therefore 
(5.36) is indeed invertible. This implies that $\operatorname{top}\left(\bar{a}_{\lambda}^{*}(L)\right)$ has a unique constituent in $\operatorname{Irr}\left(\mathcal{O}\left(\Gamma_{n+m r}\right)\right)_{0, r}$. Indeed, otherwise we would have a surjective map

$$
\bar{a}_{\lambda}^{*}(L) \rightarrow L^{\prime} \oplus L^{\prime \prime}, \quad L^{\prime}, L^{\prime \prime} \in \operatorname{Irr}\left(\mathcal{O}\left(\Gamma_{n+m r}\right)\right)_{0, r},
$$

yielding a surjective map

$$
\bar{L}_{\lambda} \otimes L=\Psi\left(\bar{a}_{\lambda}^{*}(L)\right) \rightarrow \Psi\left(L^{\prime}\right) \oplus \Psi\left(L^{\prime \prime}\right)=\left(\bar{L}_{\lambda} \otimes L\right)^{\oplus 2} .
$$

This is absurd.

Definition 5.32. For $\lambda \in \mathcal{P}_{r}$ and $L \in \operatorname{PI}(\mathcal{O}(\Gamma))$ we define $\tilde{a}_{\lambda}(L)$ to be the unique constituent of $\operatorname{top}\left(\bar{a}_{\lambda}^{*}(L)\right)$ in $\operatorname{Irr}(\mathcal{O}(\Gamma))_{0, r}$.

Proposition 5.33. For $L \in \operatorname{Irr}(\mathcal{O}(\Gamma))_{0, r}$ there is $L^{\prime} \in \operatorname{PI}(\mathcal{O}(\Gamma)), \lambda \in \mathcal{P}_{r}$ such that $\tilde{a}_{\lambda}\left(L^{\prime}\right) \simeq L$. In other words, there is a surjective map

$$
\operatorname{PI}(\mathcal{O}(\Gamma)) \times \mathcal{P}_{r} \rightarrow \operatorname{Irr}(\mathcal{O}(\Gamma))_{0, r}, \quad\left(L^{\prime}, \lambda\right) \mapsto \tilde{a}_{\lambda}\left(L^{\prime}\right)
$$

Proof. By Proposition 5.18 the module $L$ is primitive if and only if $r=0$. Thus we can assume that $r>0$, i.e., that $a_{*}(L) \neq 0$ by Corollary 3.19, else the claim is obvious. Now, we first claim that there is a module $L_{1} \in \operatorname{Irr}(\mathcal{O}(\Gamma))_{0, r-1}$ with a surjective morphism $\bar{a}^{*}\left(L_{1}\right) \rightarrow L$. Indeed, since $a_{*}(L) \neq 0$, the adjunction map $\epsilon: a^{*}\left(a_{*}(L)\right) \rightarrow L$ is nonzero; hence it is surjective. Hence, there is a constituent $L_{1}$ of $a_{*}(L)$ such that $\epsilon$ yields a surjective morphism $a^{*}\left(L_{1}\right) \rightarrow L$. We have the following lemma whose proof is postponed to the end of the section.

Lemma 5.34. If $L \in \operatorname{Irr}(\mathcal{O}(\Gamma))_{0, r}$ and $L_{1}$ is a constituent of $a_{*}(L)$ such that $a^{*}\left(L_{1}\right)$ maps onto $L$, then $L_{1} \in \operatorname{Irr}(\mathcal{O}(\Gamma))_{0, r-1}$.

Fix the integer $n$ such that $L_{1} \in \operatorname{Irr}\left(\mathcal{O}\left(\Gamma_{n}\right)\right)$. Then $\bar{\xi}_{1}$ acts on $a^{*}\left(L_{1}\right)$ as the operator

$$
\mathcal{O}_{z_{n+m}}\left(a^{*}\left(L_{1}\right)\right) \circ a^{*}\left(\mathcal{O}_{z_{n}}\left(L_{1}\right)\right)^{-1} \text {. }
$$

The second factor is a scalar because $L_{1}$ is a simple module. Hence $x_{1}$ acts on $a^{*}\left(L_{1}\right)$ as an element of the center of $\mathcal{O}\left(\Gamma_{n+m}\right)$; see (5.32). Therefore, since $L$ is simple and since the operator $x_{1}$ on $a^{*}\left(L_{1}\right)$ is nilpotent by Proposition 5.20, the operator $x_{1}$ is 0 on $L$. Thus the map $a^{*}\left(L_{1}\right) \rightarrow L$ factors to a surjective morphism

$$
\epsilon_{1}: \bar{a}^{*}\left(L_{1}\right) \rightarrow L \text {. }
$$

This proves the claim.

Now, assume that for $0<k<r$ there is a module $L_{k} \in \operatorname{Irr}(\mathcal{O}(\Gamma))_{0, r-k}$ with a surjective homomorphism

$$
\epsilon_{k}: \overline{\left(a^{*}\right)^{k}}\left(L_{k}\right) \rightarrow L, \quad \overline{\left(a^{*}\right)^{k}}\left(L_{k}\right)=\left(a^{*}\right)^{k}\left(L_{k}\right) / \sum_{i} x_{i}\left(a^{*}\right)^{k}\left(L_{k}\right) .
$$

By the claim above, there is a module $L_{k+1} \in \operatorname{Irr}(\mathcal{O}(\Gamma))_{0, r-k-1}$ with a surjective homomorphism

$$
\bar{a}^{*}\left(L_{k+1}\right) \rightarrow L_{k}
$$

Applying the functor $\left(a^{*}\right)^{k}$, which is exact, we get a surjective map

$$
\left(a^{*}\right)^{k} \bar{a}^{*}\left(L_{k+1}\right) \rightarrow\left(a^{*}\right)^{k}\left(L_{k}\right) .
$$

Taking the quotient by the action of $x_{2}, \ldots, x_{k}, x_{k+1}$, it yields a surjective map

$$
\left(a^{*}\right)^{k} \bar{a}^{*}\left(L_{k+1}\right) / \sum_{i=2}^{k+1} x_{i}\left(a^{*}\right)^{k} \bar{a}^{*}\left(L_{k+1}\right) \rightarrow \overline{\left(a^{*}\right)^{k}}\left(L_{k}\right) .
$$


Now, since $a^{*}$ is exact, we have

$$
\left(a^{*}\right)^{k} \bar{a}^{*}\left(L_{k+1}\right)=\left(a^{*}\right)^{k+1}\left(L_{k+1}\right) / x_{1}\left(a^{*}\right)^{k+1}\left(L_{k+1}\right) .
$$

Therefore we get a surjective map

$$
\overline{\left(a^{*}\right)^{k+1}}\left(L_{k+1}\right)=\left(a^{*}\right)^{k+1}\left(L_{k+1}\right) / \sum_{i=1}^{k+1} x_{i}\left(a^{*}\right)^{k} \bar{a}^{*}\left(L_{k+1}\right) \rightarrow \overline{\left(a^{*}\right)^{k}}\left(L_{k}\right) .
$$

Composing it with $\epsilon_{k}$ we get a surjective homomorphism

$$
\epsilon_{k+1}: \overline{\left(a^{*}\right)^{k+1}}\left(L_{k+1}\right) \rightarrow L
$$

By induction, this yields a module $L_{r} \in \operatorname{Irr}(\mathcal{O}(\Gamma))_{0,0}$ with a surjective homomorphism

$$
\epsilon_{r}: \overline{\left(a^{*}\right)^{r}}\left(L_{r}\right) \rightarrow L
$$

Then we have $L_{r} \in \operatorname{PI}(\mathcal{O}(\Gamma))$ by Proposition 5.18, and there is $\lambda \in \mathcal{P}_{r}$ such that $\bar{a}_{\lambda}^{*}\left(L_{r}\right)$ maps onto $L$. The proposition follows from Proposition 5.31 .

Proof of Lemma 5.34. Fix $i, j \geqslant 0$ such that $L_{1} \in \operatorname{Irr}(\mathcal{O}(\Gamma))_{i, j}$. By Proposition 5.15. since $E(L)=0$ we have $E a_{*}(L)=0$. Hence $E\left(L_{1}\right)=0$ by Proposition 3.3 . Thus $i=0$ by Corollary 3.19. So, by Proposition 5.30 we have $a^{*}\left(L_{1}\right) \in F_{0, j+1}(\Gamma)$. Since $a^{*}\left(L_{1}\right)$ maps onto $L$, we also have $[L] \in F_{0, j+1}(\Gamma)$. Since $L \in \operatorname{Irr}(\mathcal{O}(\Gamma))_{0, r}$ this implies that $r \leqslant j+1$ by Remark 3.11 .

Now, we prove that $j+1 \leqslant r$. Fix $n \geqslant 1$ such that $L \in \mathcal{O}\left(\Gamma_{n}\right)$. Recall that

$$
a_{*}(L)=\operatorname{Hom}_{\mathcal{O}\left(\mathfrak{S}_{m}\right)}\left(L_{(m)},{ }^{\mathcal{O}} \operatorname{Res}_{n-m, m}(L)\right) .
$$

Thus there is an obvious inclusion

$$
a_{*}(L) \otimes L_{(m)} \subset{ }^{\mathcal{O}} \operatorname{Res}_{n-m, m}(L) .
$$

Hence, since $L_{1}$ is a constituent of $a_{*}(L)$, the module $L_{1} \otimes L_{(m)}$ is a constituent of ${ }^{\mathcal{O}} \operatorname{Res}_{n-m, m}(L)$. Let us abbreviate

$$
W^{\prime}=\Gamma_{l,\left(m^{j}\right)}, \quad l=n-(j+1) m,
$$

regarded as a subgroup of $\Gamma_{n-m}$. Then $W^{\prime} \times \mathfrak{S}_{m} \subset \Gamma_{n-m} \times \mathfrak{S}_{m}$ in the obvious way. Since $L_{1} \in \operatorname{Irr}\left(\mathcal{O}\left(\Gamma_{n-m}\right)\right)_{0, j}$, we have

$$
\operatorname{Supp}\left(L_{1} \otimes L_{(m)}\right)=X_{W^{\prime} \times \mathfrak{S}_{m}, \mathbb{C}^{n-m} \times \mathbb{C}_{0}^{m}} .
$$

By Proposition 2.2 applied to the module $M=L$, we also have

$$
\operatorname{Supp}\left(L_{1} \otimes L_{(m)}\right)=X_{W_{1}, \mathbb{C}^{n-m} \times \mathbb{C}_{0}^{m}},
$$

where $W_{1}$ is a parabolic subgroup of $\Gamma_{n-m, m}$ containing a subgroup $\Gamma_{n}$-conjugate to $\Gamma_{n-m r,\left(m^{r}\right)}$. Hence we have $F_{0, j+1}\left(\Gamma_{n}\right) \subset F_{0, r}\left(\Gamma_{n}\right)$. Therefore we have $j+1 \leqslant r$ by Remark 3.11 .

\section{The filtration of the Fock space and Etingof's COnjecture}

Recall that $[\mathcal{O}(\Gamma)]$ is identified with the Fock space $\mathcal{F}_{m, \ell}^{(s)}$ via the map (5.20). The aim of this section is to identify the filtration on $[\mathcal{O}(\Gamma)]$ defined in Section 3.10 in terms of supports of irreducible modules, with a filtration on the Fock space given by representation-theoretic tools. We'll use the following notation: $n, m, j, i$ are integers with $n \geqslant 0, m>2, i, j \geqslant 0$ and $i=n-l-j m$. 
6.1. The representation-theoretic interpretation of $F_{0,0}(\Gamma)$. The goal of this section is to give a representation-theoretic interpretation of $F_{0,0}(\Gamma)$ using the actions of $\widehat{\mathfrak{s l}}_{m}$ and $\mathfrak{H}$ on $[\mathcal{O}(\Gamma)]$ defined in the previous sections. Note that the set $\operatorname{Irr}(\mathcal{O}(\Gamma))_{0,0}$ is a basis of the $\mathbb{C}$-vector space $F_{0,0}(\Gamma)$. Further, we have proved that $\operatorname{Irr}(\mathcal{O}(\Gamma))_{0,0}=\operatorname{PI}(\mathcal{O}(\Gamma))$ in Proposition 5.18, Recall that the operators $b_{r}^{\prime}, r \geqslant 1$, on $\mathcal{F}_{m, \ell}^{(s)}$ given in Section 4.6 act on $[\mathcal{O}(\Gamma)]$ via the map (5.20).

Lemma 6.1. For $L \in \operatorname{Irr}(\mathcal{O}(\Gamma))$ we have $L \in \operatorname{PI}(\mathcal{O}(\Gamma))$ if and only if $E([L])=$ $b_{r}^{\prime}([L])=0$ in $[\mathcal{O}(\Gamma)]$ for all $r \geqslant 1$.

Proof. It is enough to prove that for $L \in \operatorname{PI}(\mathcal{O}(\Gamma))$ we have $b_{r}^{\prime}(L)=0$ for all $r \geqslant 1$. A direct summand of the zero object is zero in any additive category. Further, for $L \in \operatorname{PI}(\mathcal{O}(\Gamma))$ we have $\left(R a_{*}\right)^{r}(L)=0$ for $r \geqslant 1$. Thus we also have $R a_{\lambda, *}(L)=0$ for all $\lambda \in \mathcal{P}$ by Proposition 5.4. By Proposition 5.13 the map (5.20) identifies the $\mathbb{C}$-linear operator $R a_{\lambda, *}$ on $[\mathcal{O}(\Gamma)]$ with the action of $b_{S_{\lambda}}^{\prime}$ on $\mathcal{F}_{m, \ell}^{(s)}$ given in Section 4.6. This proves the lemma.

In particular the lemma yields an inclusion

$$
F_{0,0}(\Gamma) \subset\left\{x \in[\mathcal{O}(\Gamma)]: e_{q}(x)=b_{r}^{\prime}(x)=0, \forall q, r\right\} .
$$

However it is not obvious that the right-hand side is spanned by classes of irreducible objects of $\mathcal{O}(\Gamma)$. This follows indeed from the next proposition.

Proposition 6.2. We have

$$
\left\{x \in[\mathcal{O}(\Gamma)]: e_{q}(x)=b_{r}^{\prime}(x)=0, \forall q, r\right\}=F_{0,0}(\Gamma) .
$$

Proof. Consider the set

$$
F_{0,0}(\Gamma)^{\prime}=\left\{x \in F_{0, \bullet}(\Gamma): b_{r}^{\prime}(x)=0, \forall r \geqslant 1\right\} .
$$

By Corollary 3.19 it is enough to prove that

$$
F_{0,0}(\Gamma)=F_{0,0}(\Gamma)^{\prime} .
$$

We have

$$
F_{0,0}(\Gamma)^{\prime}=\bigoplus_{n \geqslant 0} F_{0,0}\left(\Gamma_{n}\right)^{\prime}, \quad F_{0,0}\left(\Gamma_{n}\right)^{\prime}=F_{0,0}(\Gamma)^{\prime} \cap F_{0, \bullet}\left(\Gamma_{n}\right) .
$$

The actions of $\widehat{\mathfrak{s l}}_{m}$ and $\mathfrak{H}$ on $\mathcal{F}_{m, \ell}^{(s)}$ commute with each other. Thus, by Corollary 3.19 the $\mathbb{C}$-vector space $F_{0, \bullet}(\Gamma)$ is identified with an $\mathfrak{H}$-submodule of $\mathcal{F}_{m, \ell}^{(s)}$ via the map (5.20), and we have

$$
\sum_{n \geqslant 0} \operatorname{dim}\left(F_{0, \bullet}\left(\Gamma_{n}\right)\right) \cdot t^{n}=\sum_{n \geqslant 0} \sharp \operatorname{Irr}\left(\mathcal{O}\left(\Gamma_{n}\right)\right)_{0, \bullet} \cdot t^{n} .
$$

The representation theory of $\mathfrak{H}$ yields the following formula in $\mathbb{Z}[[t]]$ :

$$
\left(\sum_{k \geqslant 0} \operatorname{dim}\left(F_{0,0}\left(\Gamma_{k}\right)^{\prime}\right) \cdot t^{k}\right)\left(\sum_{r \geqslant 0} \sharp \mathcal{P}_{r} \cdot t^{m r}\right)=\sum_{n \geqslant 0} \operatorname{dim}\left(F_{0, \bullet}\left(\Gamma_{n}\right)\right) \cdot t^{n} .
$$

Finally, Proposition 5.33 yields a surjective map

$$
\operatorname{PI}\left(\mathcal{O}\left(\Gamma_{k}\right)\right) \times \mathcal{P}_{r} \rightarrow \operatorname{Irr}\left(\mathcal{O}\left(\Gamma_{n}\right)\right)_{0, r}, \quad(L, \lambda) \mapsto \tilde{a}_{\lambda}(L)
$$


for $k, r \geqslant 0$ such that $n=k+m r$. From (6.1) and (6.3) we get

$$
\left(\sum_{k \geqslant 0} \sharp \operatorname{PI}\left(\mathcal{O}\left(\Gamma_{k}\right)\right) \cdot t^{k}\right)\left(\sum_{r \geqslant 0} \sharp \mathcal{P}_{r} \cdot t^{m r}\right)-\sum_{n \geqslant 0} \operatorname{dim}\left(F_{0, \bullet}\left(\Gamma_{n}\right)\right) \cdot t^{n} \in \mathbb{N}[[t]] .
$$

By Corollary 3.19 and Lemma 6.1 we have $\operatorname{PI}\left(\mathcal{O}\left(\Gamma_{k}\right)\right) \subset F_{0,0}\left(\Gamma_{k}\right)^{\prime}$; hence we have

$$
\sharp \mathrm{PI}\left(\mathcal{O}\left(\Gamma_{k}\right)\right) \leqslant \operatorname{dim}\left(F_{0,0}\left(\Gamma_{k}\right)^{\prime}\right) .
$$

Therefore, comparing (6.2) and (6.4), we get the equality

$$
\sharp \operatorname{PI}\left(\mathcal{O}\left(\Gamma_{k}\right)\right)=\operatorname{dim}\left(F_{0,0}\left(\Gamma_{k}\right)^{\prime}\right) .
$$

In other words $\operatorname{PI}\left(\mathcal{O}\left(\Gamma_{k}\right)\right)$ is a basis of $F_{0,0}\left(\Gamma_{k}\right)^{\prime}$. Since $\operatorname{PI}\left(\mathcal{O}\left(\Gamma_{k}\right)\right)$ is a basis of $F_{0,0}\left(\Gamma_{k}\right)$ by Proposition 5.18, we also have

$$
F_{0,0}\left(\Gamma_{k}\right)=F_{0,0}\left(\Gamma_{k}\right)^{\prime} .
$$

Remark 6.3. The proof of Proposition 6.2 and Corollary 3.19 imply that the map (6.3) yields a bijection

$$
\operatorname{PI}\left(\mathcal{O}\left(\Gamma_{k}\right)\right) \times \mathcal{P}_{r} \rightarrow \operatorname{Irr}\left(\mathcal{O}\left(\Gamma_{n}\right)\right)_{0, r}, \quad(L, \lambda) \mapsto \tilde{a}_{\lambda}(L)
$$

for $k, r \geqslant 0$ such that $n=k+m r$. Note that Proposition 5.18 yields

$$
\operatorname{PI}\left(\mathcal{O}\left(\Gamma_{k}\right)\right)=\operatorname{Irr}\left(\mathcal{O}\left(\Gamma_{k}\right)\right)_{0,0} \text {. }
$$

6.2. The representation-theoretic grading on $[\mathcal{O}(\Gamma)]$. Using the actions of the Lie algebras $\mathfrak{H}$ and $\widehat{\mathfrak{s l}}_{m}$ we define another grading

$$
[\mathcal{O}(\Gamma)]=\bigoplus_{i, j \geqslant 0}[\mathcal{O}(\Gamma)]_{i, j}
$$

as follows. First, let us consider the level $m \ell$ Casimir operator

$$
\partial=\frac{1}{m \ell} \sum_{r \geqslant 1} b_{r} b_{r}^{\prime} ;
$$

see (4.2). Under the map (5.20) this formal sum defines a diagonalisable $\mathbb{C}$-linear operator on $[\mathcal{O}(\Gamma)]$. For any integer $j$, let $[\mathcal{O}(\Gamma)]_{\bullet, j}$ be the eigenspace of $\partial$ with the eigenvalue $j$. Note that $[\mathcal{O}(\Gamma)]_{\bullet, j}=0$ if $j<0$. Next, given an integer $i \geqslant 0$ we define $[\mathcal{O}(\Gamma)]_{i, \bullet}$ to be the image of

$$
\bigoplus_{\mu, \alpha} V_{\mu}^{\widehat{\mathfrak{s l}}_{m}}[\mu-\alpha] \otimes \operatorname{Hom}_{\widehat{\mathfrak{s l}}_{m}}\left(V_{\mu}^{\widehat{\mathfrak{s l}}_{m}},[\mathcal{O}(\Gamma)]\right)
$$

under the canonical maps

$$
V_{\mu}^{\widehat{\mathfrak{s l}}_{m}} \otimes \operatorname{Hom}_{\widehat{\mathfrak{s l}}_{m}}\left(V_{\mu}^{\widehat{\mathfrak{s l}}_{m}},[\mathcal{O}(\Gamma)]\right) \rightarrow[\mathcal{O}(\Gamma)]
$$

Here the sum runs over all $\alpha$, which is a sum of $i$ affine simple roots of $\widehat{\mathfrak{s l}}_{m}$, and over all dominant affine weights $\mu$ of $\widehat{\mathfrak{s l}}_{m}$. Recall also that $V_{\mu}^{\widehat{\mathfrak{s l}}_{m}}[\mu-\alpha]$ denotes the $(\mu-\alpha)$-weight subspace of $V_{\mu}^{\widehat{\mathfrak{s} l}}$. If $i<0$ we set $[\mathcal{O}(\Gamma)]_{i, \bullet}=0$.

Definition 6.4. We define a grading on $[\mathcal{O}(\Gamma)]$ by the following formula:

$$
[\mathcal{O}(\Gamma)]_{i, j}=[\mathcal{O}(\Gamma)]_{i, \bullet} \cap[\mathcal{O}(\Gamma)]_{\bullet, j}, \quad\left[\mathcal{O}\left(\Gamma_{n}\right)\right]_{i, j}=[\mathcal{O}(\Gamma)]_{i, j} \cap\left[\mathcal{O}\left(\Gamma_{n}\right)\right] .
$$

The following proposition compares this grading with the grading $\operatorname{gr}_{i, j}(\Gamma)$ associated with the filtration by the support introduced in Section 3.10 . 
Proposition 6.5. We have $\operatorname{dim}\left[\mathcal{O}\left(\Gamma_{n}\right)\right]_{i, j}=\operatorname{dim} g r_{i, j}\left(\Gamma_{n}\right)$ for all $n, i, j \geqslant 0$.

Proof. The vector space $[\mathcal{O}(\Gamma)]_{0, \bullet}$ is an $\mathfrak{H}$-submodule of $[\mathcal{O}(\Gamma)]$. Thus it is preserved by the linear operator $\partial$, and $[\mathcal{O}(\Gamma)]_{0, j}$ is the eigenspace with the eigenvalue $j$. Since the $\mathfrak{H}$-action on $[\mathcal{O}(\Gamma)]_{0, \bullet}$ has the level $m \ell$, we have $\left[\partial, b_{j}\right]=j b_{j}$ for all $j>0$. Next, we have

$$
[\mathcal{O}(\Gamma)]_{0, \bullet}=F_{0, \bullet}(\Gamma), \quad[\mathcal{O}(\Gamma)]_{0,0}=F_{0,0}(\Gamma)
$$

by Corollary 3.19 and Proposition 6.2, Further, the $\mathfrak{H}$-action yields an isomorphism

$$
U^{-}(\mathfrak{H})_{j} \otimes[\mathcal{O}(\Gamma)]_{0,0}=[\mathcal{O}(\Gamma)]_{0, j} .
$$

By Remark 6.3, for $n=k+m j$ we have a bijection

$$
\operatorname{Irr}\left(\mathcal{O}\left(\Gamma_{k}\right)\right)_{0,0} \times \mathcal{P}_{j} \rightarrow \operatorname{Irr}\left(\mathcal{O}\left(\Gamma_{n}\right)\right)_{0, j}, \quad(L, \lambda) \mapsto \tilde{a}_{\lambda}(L) .
$$

Thus the isomorphism (6.7) yields the following equality:

$$
\operatorname{dim}\left[\mathcal{O}\left(\Gamma_{n}\right)\right]_{0, j}=\sharp \operatorname{Irr}\left(\mathcal{O}\left(\Gamma_{n}\right)\right)_{0, j} .
$$

Now, to compare $\operatorname{dim}\left[\mathcal{O}\left(\Gamma_{n}\right)\right]_{i, j}$ and $\sharp \operatorname{Irr}\left(\mathcal{O}\left(\Gamma_{n}\right)\right)_{i, j}$ for any $i \geqslant 0$, we need some tools from canonical bases. Since the integrable $\widehat{\mathfrak{s l}}_{m}$-module $[\mathcal{O}(\Gamma)]$ is not simple, the choice of a canonical basis of this module depends on a choice of a basis of $[\mathcal{O}(\Gamma)]_{0, \bullet}$. The general theory of canonical bases yields a bijection $G$ between the canonical basis of $[\mathcal{O}(\Gamma)]$ and its crystal basis, the latter being identified with $\operatorname{Irr}(\mathcal{O}(\Gamma))$ by Proposition 3.3. The bijection $\mathrm{G}$ is such that a basis of $[\mathcal{O}(\Gamma)]_{0, \bullet}$ is given by

$$
\left\{\mathrm{G}(L): \tilde{e}_{q}(L)=0, \forall q\right\} .
$$

By Corollary 3.19 we have

$$
\begin{aligned}
\left\{L \in \operatorname{Irr}(\mathcal{O}(\Gamma)): \tilde{e}_{q}(L)=0, \forall q\right\} & =\operatorname{Irr}(\mathcal{O}(\Gamma))_{0, \bullet} \\
& =\left\{\tilde{a}_{\lambda}(L): \forall \lambda \in \mathcal{P}, \forall L \in \operatorname{Irr}(\mathcal{O}(\Gamma))_{0,0}\right\} .
\end{aligned}
$$

We'll choose the canonical basis of $[\mathcal{O}(\Gamma)]$ such that

$$
\mathrm{G}\left(\tilde{a}_{\lambda}(L)\right)=a_{\lambda}^{*}(L), \quad \forall \lambda \in \mathcal{P}, \quad \forall L \in \operatorname{Irr}(\mathcal{O}(\Gamma))_{0,0} .
$$

Then the set $\left\{\mathrm{G}(L): L \in \operatorname{Irr}(\mathcal{O}(\Gamma))_{0, j}\right\}$ is a basis of $[\mathcal{O}(\Gamma)]_{0, j}$ by (6.7) and (6.8). The $\widehat{\mathfrak{s l}}_{m}$-action on $[\mathcal{O}(\Gamma)]$ commutes with the operator $\partial$. Thus $[\mathcal{O}(\Gamma)] \bullet$ $\widehat{\mathfrak{s l}}_{m}$-module and the $\widehat{\mathfrak{s l}}_{m}$-action yields a surjective $\mathbb{C}$-linear map

$$
U^{-}\left(\widehat{\mathfrak{s l}}_{m}\right)_{i} \otimes[\mathcal{O}(\Gamma)]_{0, j} \rightarrow[\mathcal{O}(\Gamma)]_{i, j}
$$

For weight reasons, the crystal of $[\mathcal{O}(\Gamma)]$ decomposes in the following way:

$$
\operatorname{Irr}(\mathcal{O}(\Gamma))=\bigsqcup_{i, j \geqslant 0} \operatorname{Irr}(\mathcal{O}(\Gamma))_{i, j}^{\prime}, \quad \operatorname{Irr}(\mathcal{O}(\Gamma))_{i, j}^{\prime}=\left\{L \in \operatorname{Irr}(\mathcal{O}(\Gamma)): G(L) \in[\mathcal{O}(\Gamma)]_{i, j}\right\}
$$

Since $\left\{\mathrm{G}(L): L \in \operatorname{Irr}(\mathcal{O}(\Gamma))_{0, j}\right\}$ is a basis of $[\mathcal{O}(\Gamma)]_{0, j}$, we have

$$
\operatorname{Irr}(\mathcal{O}(\Gamma))_{0, j}^{\prime}=\operatorname{Irr}(\mathcal{O}(\Gamma))_{0, j}
$$

Next $\operatorname{Irr}(\mathcal{O}(\Gamma))_{\bullet, j}^{\prime}$ is the union of connected components of $\operatorname{Irr}(\mathcal{O}(\Gamma))$ whose highest weight vector is in $\operatorname{Irr}(\mathcal{O}(\Gamma))_{0, j}^{\prime}$, and by Corollary 3.18, the set $\operatorname{Irr}(\mathcal{O}(\Gamma))_{\bullet, j}$ is the union of connected components of $\operatorname{Irr}(\mathcal{O}(\Gamma))$ whose highest weight vector is in $\operatorname{Irr}(\mathcal{O}(\Gamma))_{0, j}$. Thus, for all $n$ we have

$$
\operatorname{Irr}\left(\mathcal{O}\left(\Gamma_{n}\right)\right)_{\bullet, j}^{\prime}=\operatorname{Irr}\left(\mathcal{O}\left(\Gamma_{n}\right)\right)_{\bullet, j}
$$


By Corollary 3.18 and (6.10), for all $i$ we also have the inclusion

$$
\operatorname{Irr}\left(\mathcal{O}\left(\Gamma_{n}\right)\right)_{i, j}^{\prime} \subset \operatorname{Irr}\left(\mathcal{O}\left(\Gamma_{n}\right)\right)_{i, j}
$$

Thus (6.11) is indeed an equality. By definition, we have

$$
\operatorname{dim} \operatorname{gr}_{i, j}\left(\Gamma_{n}\right)=\sharp \operatorname{Irr}\left(\mathcal{O}\left(\Gamma_{n}\right)\right)_{i, j}, \quad \operatorname{dim}\left[\mathcal{O}\left(\Gamma_{n}\right)\right]_{i, j}=\sharp \operatorname{Irr}\left(\mathcal{O}\left(\Gamma_{n}\right)\right)_{i, j}^{\prime} .
$$

Thus the corollary is proved.

Remark 6.6. Recall that $\operatorname{gr}_{i, j}(\Gamma)$ is identified with the subspace of $[\mathcal{O}(\Gamma)]$ spanned by $\operatorname{Irr}(\mathcal{O}(\Gamma))_{i, j}$; see Section 3.10 . Proposition 6.5 does not imply that $[\mathcal{O}(\Gamma)]_{i, j}=$ $\operatorname{gr}_{i, j}(\Gamma)$. Indeed, the classes of the modules in $\operatorname{Irr}(\mathcal{O}(\Gamma))_{i, j}$ may not lie in $[\mathcal{O}(\Gamma)]_{i, j}$. However, since

$$
[\mathcal{O}(\Gamma)]_{0,0}=\left\{x \in[\mathcal{O}(\Gamma)]: e_{q}(x)=b_{r}^{\prime}(x)=0, \forall q, r\right\},
$$

we do have $[\mathcal{O}(\Gamma)]_{0,0}=\operatorname{gr}_{0,0}(\Gamma)$ by Proposition 6.2 .

6.3. Etingof's conjecture. In this section we allow $h, h_{p}$ to be arbitrary complex numbers with $\sum_{p \in \mathbb{Z}_{\ell}} h_{p}=1$. Let $\alpha_{p, q}$ be the root of the elementary matrix $e_{p, q}$ in $\mathfrak{g l}_{\ell}$. Recall that $\omega_{0}, \omega_{1}, \ldots, \omega_{\ell-1}$ are the affine fundamental weights. Fix a level 1 weight

$$
\Lambda=\sum_{p} h_{p} \omega_{p}
$$

Definition 6.7. Let $\tilde{\mathfrak{a}}_{\Lambda}$ be the Lie subalgebra of $\tilde{\mathfrak{g l}}_{\ell}$ spanned by $\mathbf{1}, D$ and the elements $e_{p, q} \otimes \varpi^{r}$ with $p, q=1,2, \ldots, \ell$ and $r \in \mathbb{Z}$ such that $\left\langle\Lambda, \alpha_{p, q}\right\rangle-h r \in \mathbb{Z}$. We abbreviate $\tilde{\mathfrak{a}}=\tilde{\mathfrak{a}}_{\Lambda}$ and $\hat{\mathfrak{a}}=\tilde{\mathfrak{a}} \cap \widehat{\mathfrak{g l}}_{\ell}$.

We define the set of positive real roots of $\tilde{\mathfrak{a}}$ to be the set $\Delta_{+}^{\hat{\mathfrak{a}}}$ consisting of the real roots of $\widehat{\mathfrak{g l}}_{\ell}$ of the form

$$
\alpha-\frac{\langle\Lambda, \alpha+r \delta\rangle}{h} \delta
$$

where $\alpha$ is a root of $\mathfrak{g l}_{\ell}$ and $\alpha+r \delta$ is a positive real root of $\widehat{\mathfrak{g l}}_{\ell}$. Let $P_{+}^{\tilde{a}}$ be the set of dominant integral weights for $\tilde{\mathfrak{a}}$, i.e., the set of integral weights $\lambda$ of $\mathfrak{g l}_{\ell}$ such that $\langle\lambda, \beta\rangle \geqslant 0$ for all $\beta \in \Delta_{+}^{\hat{\mathfrak{a}}}$. For $\mu \in P_{+}^{\tilde{\mathfrak{a}}}$ let $V_{\mu}^{\tilde{\mathfrak{a}}}$ be the irreducible integrable $\tilde{\mathfrak{a}}$-module of highest weight $\mu$. We'll say that a nonzero vector of an $\tilde{\mathfrak{a}}$-module is primitive for $\tilde{\mathfrak{a}}$ (or $\tilde{\mathfrak{a}}$-primitive) if it is a weight vector whose weight belongs to $P_{+}^{\tilde{\mathfrak{a}}}$, and if it is killed by the action of the weight vectors of $\tilde{\mathfrak{a}}$ whose weights are positive roots of $\tilde{\mathfrak{a}}$. Now, let $h, h_{p}$ be the parameters of the $\mathbb{C}$-algebra $H\left(\Gamma_{n}\right)$ for each $n>0$. Assume that $h$ is a rational number with the denominator $m>1$. The elements of $\mathfrak{H}$ can be regarded as elements of $\widetilde{\mathfrak{g l}}_{\ell}$ as in (4.3). We have $b_{m r}, b_{m r}^{\prime} \in \tilde{\mathfrak{a}}$ for each $r>0$. The formal sum

$$
\partial_{m}=\frac{1}{m \ell} \sum_{r \geqslant 1} b_{m r} b_{m r}^{\prime}
$$

acts on every $\tilde{\mathfrak{a}}$-module $V_{\mu}^{\tilde{\mathfrak{a}}}$. We'll call $\partial_{m}$ the $m$-th Casimir operator of $\tilde{\mathfrak{g l}}_{\ell}$. For any weight $\lambda$ and any integer $j$ we denote by $V_{\mu}^{\tilde{a}}[\lambda, j]$ the subspace of weight $\lambda$ and eigenvalue $j$ of $\partial_{m}$. We are interested in the following conjecture [11, conj. 6.7]. 
Conjecture 6.8. There exists an isomorphism of $\mathbb{C}$-vector spaces

$$
g r_{i, j}\left(\Gamma_{n}\right)=\bigoplus_{\mu} V_{\mu}^{\tilde{\mathfrak{a}}}\left[\omega_{0}-n \delta, j\right] \otimes \operatorname{Hom}_{\tilde{\mathfrak{a}}}\left(V_{\mu}^{\tilde{\mathfrak{a}}}, V_{\omega_{0}}^{\tilde{\mathfrak{g}} l_{\ell}}\right),
$$

where the sum is over all weights $\mu \in P_{+}^{\tilde{a}}$ such that $\langle\mu, \mu\rangle=-2 i$.

Remark 6.9. If $\Lambda=\omega_{0}$, then we have

$$
\tilde{\mathfrak{a}}_{\omega_{0}}=\left(\mathfrak{g l}_{\ell} \otimes \mathbb{C}\left[\varpi^{m}, \varpi^{-m}\right]\right) \oplus \mathbb{C} \mathbf{1} \oplus \mathbb{C} D,
$$

and the map (6.19) yields a Lie algebra isomorphism $\tilde{\mathfrak{a}}_{\omega_{0}}=\tilde{\mathfrak{g l}}_{\ell}$.

Theorem 6.10. Assume that $m>2$ and that $h, h_{p}$ are as in (3.9), i.e.,

$$
h=-1 / m, \quad h_{p}=\left(s_{p+1}-s_{p}\right) / m, \quad s_{q} \in \mathbb{Z}, \quad p \neq 0 .
$$

Then Conjecture 6.8 holds.

The proof will be given in Section 6.6. In the next two subsections we give some reminders that will be useful for the proof.

6.4. Reminder on the level-rank duality. For $\lambda \in \mathbb{Z}^{\ell}$ we consider the weight $\hat{\gamma}(\lambda, m) \in P^{\widehat{\mathfrak{s} l_{\ell}}}$ defined in (4.16) and the weight

$$
\tilde{\gamma}(\lambda, m)=\hat{\gamma}(\lambda, m)-\Delta(\lambda, m) \delta \in P^{\widetilde{\mathfrak{s l}}_{\ell}}
$$

see Remark 4.7. Note that the weight $\tilde{\gamma}(\lambda, m)$ is dominant if and only if

$$
\lambda \in A(\ell, m)=\left\{\left(\lambda_{1}, \lambda_{2}, \ldots, \lambda_{\ell}\right) \in \mathbb{Z}_{+}^{\ell}: \lambda_{1}-\lambda_{\ell} \leqslant m\right\} .
$$

For $d \in \mathbb{Z}$ we write

$$
A(\ell, m)_{d}=\left\{\lambda \in A(\ell, m): \sum_{p} \lambda_{p}=d\right\} .
$$

The level-rank duality yields a bijection $A(\ell, m)_{d} \rightarrow A(m, \ell)_{d}, \lambda \mapsto \lambda^{\dagger}$ such that

- we have the equality of weights

$$
\hat{\gamma}(\lambda, m)=\sum_{p=1}^{m} \omega_{\lambda_{p}^{\dagger} \bmod \ell},
$$

- we have an $\tilde{\mathfrak{s l}}_{m} \times \mathfrak{H} \times \widetilde{\mathfrak{s l}}_{\ell}$-module isomorphism

$$
\mathcal{F}_{m, \ell}^{(d)}=\bigoplus_{\lambda \in A(\ell, m)_{d}} V_{\tilde{\gamma}\left(\lambda^{\dagger}, \ell\right)}^{\tilde{\mathfrak{s l}}_{m}} \otimes V_{m \ell}^{\mathfrak{H}} \otimes V_{\tilde{\gamma}(\lambda, m)}^{\widetilde{\mathfrak{s} l} l_{\ell}}
$$

and there are highest weight vectors $v_{\tilde{\gamma}\left(\lambda^{\dagger}, \ell\right)}, v_{m \ell}, v_{\tilde{\gamma}(\lambda, m)}$ of $V_{\tilde{\gamma}\left(\lambda^{\dagger}, \ell\right)}^{\widetilde{\mathfrak{s} l}_{m}}, V_{m \ell}^{\mathfrak{H}}$, $V_{\tilde{\gamma}(\lambda, m)}^{\widetilde{\mathfrak{s}}_{\ell}}$ such that $|0, \lambda\rangle=v_{\tilde{\gamma}\left(\lambda^{\dagger}, \ell\right)} \otimes v_{m \ell} \otimes v_{\tilde{\gamma}(\lambda, m)}$ for $\lambda \in A(\ell, m)_{d}$.

See, e.g., [29, (3.19)], [35, sec. 4.2, 4.3], for details. Let $s=\left(s_{p}\right)$ be an $\ell$-charge of weight $d$. Setting $d=0$, the formula (4.17) yields the following decomposition:

$$
\mathcal{F}_{m, \ell}^{(s)}=\bigoplus_{\lambda \in A(\ell, m)_{0}} V_{\hat{\gamma}\left(\lambda^{\dagger}, \ell\right)}^{\widehat{\mathfrak{s} l_{m}}} \otimes V_{m \ell}^{\mathfrak{H}} \otimes\left(V_{\hat{\gamma}(\lambda, m)}^{\widehat{\mathfrak{s} l}(\ell}[\hat{\gamma}(s, m)]\right) .
$$

Here the bracket indicates the weight subspace for the $\widehat{\mathfrak{s l}}_{\ell}$-action of level $m$. 
6.5. Comparison of the $\tilde{\mathfrak{g l}}_{\ell}$-modules $\mathcal{F}_{m, \ell}^{(d)}$ and $V_{\omega_{d \bmod \ell}}^{\tilde{\mathfrak{g}}_{\ell}}$. The Fock space $\mathcal{F}_{m, \ell}$ can be equipped with a level 1 representation of $\widetilde{\mathfrak{g l}}_{\ell}$ in the following way. The assignment

$$
z \mapsto t^{m}, \quad \epsilon_{i} \mapsto t^{1-i}, \quad i=1,2, \ldots, m,
$$

yields a $\mathbb{C}$-linear isomorphism

$$
\begin{gathered}
V_{m, \ell}=\mathbb{C}^{m} \otimes \mathbb{C}^{\ell} \otimes \mathbb{C}\left[z, z^{-1}\right] \rightarrow \mathbb{C}^{\ell} \otimes \mathbb{C}\left[t, t^{-1}\right]=V_{\ell}, \\
u_{i+(j-1) m-k m \ell} \mapsto u_{j+(i-1) \ell-k m \ell}
\end{gathered}
$$

see (4.9), (4.14). Taking semi-infinite wedges, it yields a $\mathbb{C}$-linear isomorphism

$$
\mathcal{F}_{m, \ell} \rightarrow \mathcal{F}_{\ell}
$$

Pulling back the representation of $\widetilde{\mathfrak{g l}}_{\ell}$ on $\mathcal{F}_{\ell}$ in Section 4.5 and Remark 4.7 (with the integers $\ell, m$ in Remark 4.7 replaced by $1, \ell$ ) by (6.17) we get a level 1 action of $\tilde{\mathfrak{g l}}_{\ell}$ on $\mathcal{F}_{m, \ell}$ such that:

- For $d \in \mathbb{Z}$ the level 1 representation of $\tilde{\mathfrak{g l}}_{\ell}$ on $\mathcal{F}_{m, \ell}$ yields an isomorphism

$$
\mathcal{F}_{m, \ell}^{(d)}=V_{\omega_{d \bmod \ell}}^{\tilde{\mathfrak{g l}}_{\ell}} .
$$

- The level $m$ action of $\widehat{\mathfrak{g l}}_{\ell}$ in $\mathcal{F}_{m, \ell}$ given in Section 4.6 can be recovered from the level 1 action by composing it with the Lie algebra homomorphism

$$
\widehat{\mathfrak{g l}}_{\ell} \rightarrow \widehat{\mathfrak{g l}}_{\ell}, \quad x \otimes \varpi^{r} \mapsto x \otimes \varpi^{m r}, \quad \mathbf{1} \mapsto m \mathbf{1} .
$$

- Pulling back the level $\ell$ representation of $\mathfrak{H}$ on $\mathcal{F}_{\ell}$ in Section 4.5 by (6.17) we get a level $\ell$ action of $\mathfrak{H}$ on $\mathcal{F}_{m, \ell}$. The level $m \ell$ action of $\mathfrak{H}$ in $\mathcal{F}_{m, \ell}$ given in Section 4.6 can be recovered from the latter by composing it with the Lie algebra homomorphism

$$
b_{r} \mapsto b_{m r}, \quad b_{r}^{\prime} \mapsto b_{m r}^{\prime}, \quad \mathbf{1} \mapsto m \mathbf{1} .
$$

Hence, the action of the Casimir operator associated with the level $m \ell$ representation of $\mathfrak{H}$ on $\mathcal{F}_{m, \ell}$,

$$
\partial=\frac{1}{m \ell} \sum_{r \geqslant 1} b_{r} b_{r}^{\prime},
$$

see (4.2), is the same as the action of the $m$-th Casimir operator

$$
\partial_{m}=\frac{1}{m \ell} \sum_{r \geqslant 1} b_{m r} b_{m r}^{\prime}
$$

associated with the level $\ell$ representation of $\mathfrak{H}$ on $\mathcal{F}_{m, \ell}$.

- To a partition $\lambda$ we associate an $\ell$-quotient $\lambda^{*}$, an $\ell$-core $\lambda^{c}$ and a content polynomial

$$
c_{\lambda}(X)=\prod_{(i, j) \in \lambda}(X+c(i, j))
$$

where $c(i, j)$ is the content of the node $(i, j)$ in $\lambda$; see [27, chap. I]. In [25, sec. 2.1] a bijection $\tau$ is given from the set of $\ell$-cores to the set of $\ell$-charges of weight 0. By [35, rem. 4.2(i)] the inverse of the map (6.17) is given by

$$
\mathcal{F}_{\ell}^{(0)} \rightarrow \mathcal{F}_{m, \ell}^{(0)}, \quad|\lambda, 0\rangle \mapsto\left|\lambda^{*}, \tau\left(\lambda^{c}\right)\right\rangle .
$$


Now, the argument which is sketched in part $(b)$ of [27, ex. I.11] shows that

$$
c_{\lambda}(X)=c_{\lambda^{c}}(X) \prod_{p=0}^{\ell-1}(X+p)^{\left|\lambda^{*}\right|} \bmod \ell .
$$

Next, by Remark 4.7 the scaling element $D$ of the level 1 representation of $\widetilde{\mathfrak{g l}}_{\ell}$ on $\mathcal{F}_{m, \ell}^{(0)}$ is given by

$$
D(|\lambda, 0\rangle)=-n_{0}(\lambda)|\lambda, 0\rangle
$$

where $n_{0}(\lambda)$ is the number of 0 -nodes in $\lambda$. Note that $n_{0}(\lambda)$ is the largest power of $X$ which divides the polynomial $c_{\lambda}(X)$. Thus, comparing (6.22) and (6.23), we get the following relation:

$$
\left[D, f_{p}\right]=-f_{p},
$$

where $p=0,1, \ldots, m-1$ and the elements $f_{p}$ are the Chevalley generators of $\widehat{\mathfrak{s l}}_{m}$ which act on $\mathcal{F}_{m, \ell}^{(0)}$; see Section 3.4. Formula (6.25) should be viewed as an equality of operators on $\mathcal{F}_{m, \ell}^{(0)}$. Note also that (6.23) and (6.24) give the following formula:

$$
D(|\lambda, 0\rangle)=-\left(n_{0}\left(\lambda^{c}\right)+\left|\lambda^{*}\right|\right)|\lambda, 0\rangle .
$$

6.6. Proof of Theorem 6.10, Set

$$
\gamma=-\sum_{p=1}^{\ell-1} \frac{h_{p}}{h}\left(\omega_{p}-\omega_{0}\right)
$$

Note that by (3.9) we have $\gamma \in P^{\mathfrak{s l} l_{\ell}}$ because

$$
\gamma=\sum_{p=1}^{\ell-1}\left(s_{p+1}-s_{p}\right)\left(\omega_{p}-\omega_{0}\right) .
$$

We have $\alpha(\gamma)=-\langle\Lambda, \alpha\rangle / h$ for each root $\alpha$ of $\mathfrak{s l}_{\ell}$. Let $\bar{K}$ be the algebraic closure of the field $K=\mathbb{C}((\varpi))$. We may view $\gamma$ as the element $\gamma(\varpi)$ in $T_{\ell}(\bar{K})$. We have

$$
\tilde{\mathfrak{a}}=\operatorname{ad}(\gamma)^{-1}\left(\tilde{\mathfrak{a}}_{\omega_{0}}\right) .
$$

Next, a short computation using the standard identification of $\omega_{p}-\omega_{0}$ with the $\ell$-tuple

$$
\left(1^{p} 0^{\ell-p}\right)-p \ell^{-1}\left(1^{\ell}\right)
$$

shows that $\gamma$ belongs to $Q^{\mathfrak{s l} \ell}$ if and only if the $\ell$-charge $s$ has weight 0 , i.e., $\sum_{p \in \mathbb{Z}_{\ell}} s_{p}=0$. In this case we have $\gamma \in T_{\ell}(K)$. In other words, $\gamma$ is a cocharacter of $T_{\ell}$. Thus the element $\xi_{\gamma}$ of the affine symmetric group $\widehat{\mathfrak{S}}_{\ell}$ is well-defined.

Assume from now on that $s$ has weight 0 . To simplify the exposition, we'll only give the proof of the theorem in this case. This will be done in several steps. The proof for arbitrary weight $d$ is similar; see Remark 6.14 for more details.

Step 1. We first relate $[\mathcal{O}(\Gamma)]$ to the level one $\widehat{\mathfrak{g l}} \ell_{\ell}$-module $V_{\omega_{0}}^{\widehat{\mathfrak{g l}}_{\ell}}$. To any level one weight $\mu$ of $\widehat{\mathfrak{g l}}_{\ell}$ we associate the level $m$ weight $\mu^{\prime}$ given by

$$
\mu^{\prime}=m \omega_{0}+\sum_{p=1}^{\ell-1} \mu_{p}\left(\omega_{p}-\omega_{0}\right), \quad \text { where } \quad \mu=\omega_{0}+\sum_{p=1}^{\ell-1} \mu_{p}\left(\omega_{p}-\omega_{0}\right) .
$$


Then by (4.4) we have

$$
\hat{\gamma}(s, m)=\xi_{\gamma}^{-1}\left(\omega_{0}\right)^{\prime},
$$

where $\hat{\gamma}(s, m) \in P^{\widehat{\mathfrak{s l}}_{\ell}}$ is as in (4.16). Using this and (6.15) we get an $\widehat{\mathfrak{s l}}_{m} \times \mathfrak{H}$-module isomorphism

$$
\mathcal{F}_{m, \ell}^{(s)}=\bigoplus_{\lambda \in A(\ell, m)_{0}} V_{\hat{\gamma}\left(\lambda^{\dagger}, \ell\right)}^{\widehat{\mathfrak{s} l_{m}}} \otimes V_{m \ell}^{\mathfrak{H} \ell} \otimes\left(V_{\hat{\gamma}(\lambda, m)}^{\widehat{\mathfrak{s} \ell_{\ell}}}\left[\xi_{\gamma}^{-1}\left(\omega_{0}\right)^{\prime}\right]\right) .
$$

Thus, by (4.17), (6.18) and (6.19) we have

$$
\mathcal{F}_{m, \ell}^{(s)}=V_{\omega_{0}}^{\widehat{\mathfrak{g}}_{\ell}}\left[\xi_{\gamma}^{-1}\left(\omega_{0}\right)\right],
$$

where the bracket indicates the weight subspace for the $\widehat{\mathfrak{g l}}_{\ell^{-}}$action of level 1. Since the map (5.20) yields an isomorphism $[\mathcal{O}(\Gamma)]=\mathcal{F}_{m, \ell}^{(s)}$, we also get an isomorphism

$$
[\mathcal{O}(\Gamma)]=V_{\omega_{0}}^{\widehat{\mathfrak{g} l}}\left[\xi_{\gamma}^{-1}\left(\omega_{0}\right)\right] .
$$

Step 2. We show that for each $n$ the isomorphism (6.33) restricts to an isomorphism

$$
\left[\mathcal{O}\left(\Gamma_{n}\right)\right]=V_{\omega_{0}}^{\widetilde{\mathfrak{g}} \mathfrak{l}_{\ell}}\left[\xi_{\gamma}^{-1}\left(\omega_{0}\right)-n \delta\right]
$$

For any $\mu \in \mathcal{P}_{n}^{\ell}$ we need to compute the weight of $|\mu, s\rangle$ in the right-hand side of (6.32). By (6.22) there exists $|\lambda, 0\rangle \in \mathcal{F}_{\ell}^{(0)}$ such that $\lambda^{*}=\mu$ and $\tau\left(\lambda^{c}\right)=s$. Then by applying Remark 4.7 to the $\widetilde{\mathfrak{s l}}_{\ell}$-action on $\mathcal{F}_{\ell}^{(0)}$ and by (6.23) we see that

$$
\operatorname{wt}(|\lambda, 0\rangle)=\operatorname{wt}\left(\left|\lambda^{c}, 0\right\rangle\right)-\left|\lambda^{*}\right| \delta .
$$

In other words we have

$$
\operatorname{wt}(|\mu, s\rangle)=\operatorname{wt}(|0, s\rangle)-|\mu| \delta=\operatorname{wt}(|0, s\rangle)-n \delta .
$$

Here the symbol wt denotes the weight with respect to the level one $\tilde{\mathfrak{g l}}_{\ell}$-action. This implies the equality (6.34), because the weight of $|0, s\rangle$ is $\xi_{\gamma}^{-1}\left(\omega_{0}\right)$ by the following lemma. Note that the proofs of Lemmas 6.11, 6.12, 6.13 below are postponed to the end of the section.

Lemma 6.11. Let $\lambda \in \mathcal{P}$.

(a) If $\lambda^{c}$ is an $\ell$-core such that $\tau\left(\lambda^{c}\right)=s$, then $n_{0}\left(\lambda^{c}\right)=\frac{1}{2}\langle\gamma, \gamma\rangle$.

(b) The element $|0, s\rangle$ is an extremal weight vector of the module $\mathcal{F}_{m, \ell}^{(0)}=V_{\omega_{0}}^{\widetilde{\mathfrak{g} l_{\ell}}}$ with the weight $\xi_{\gamma}^{-1}\left(\omega_{0}\right)$.

Step 3. Recall from Proposition 6.5 that for all $i, j$ we have

$$
\operatorname{dimgr}_{i, j}\left(\Gamma_{n}\right)=\operatorname{dim}\left[\mathcal{O}\left(\Gamma_{n}\right)\right]_{i, j} .
$$

Therefore to prove the theorem we are reduced to showing that

$$
\operatorname{dim}\left[\mathcal{O}\left(\Gamma_{n}\right)\right]_{i, j}=\sum_{\mu} \operatorname{dim}\left(V_{\mu}^{\tilde{\mathfrak{a}}}\left[\omega_{0}-n \delta, j\right] \otimes \operatorname{Hom}_{\tilde{\mathfrak{a}}}\left(V_{\mu}^{\tilde{\mathfrak{a}}}, V_{\omega_{0}}^{\tilde{\mathfrak{g} l} \ell}\right)\right),
$$

where the sum is over all weights $\mu \in P_{+}^{\tilde{a}}$ such that $\langle\mu, \mu\rangle=-2 i$. 
Step 4. Recall that

$$
\left[\mathcal{O}\left(\Gamma_{n}\right)\right]_{\bullet, j}=\bigoplus_{i \geqslant 0}\left[\mathcal{O}\left(\Gamma_{n}\right)\right]_{i, j}
$$

In this step we prove that

$$
\operatorname{dim}\left[\mathcal{O}\left(\Gamma_{n}\right)\right]_{\bullet, j}=\operatorname{dim}\left(V_{\omega_{0}}^{\widetilde{\mathfrak{g} l}}\left[\omega_{0}-n \delta, j\right]\right) .
$$

Note that

$$
\operatorname{dim}\left(V_{\omega_{0}}^{\widetilde{\mathfrak{g} l_{\ell}}}\left[\omega_{0}-n \delta, j\right]\right)=\operatorname{dim}\left(V_{\omega_{0}}^{\widetilde{\mathfrak{g} l} l}\left[\xi_{\gamma}^{-1}\left(\omega_{0}\right)-n \delta, j\right]\right),
$$

because the $m$-th Casimir operator $\partial_{m}$ commutes with the $\gamma$-action on $V_{\omega_{0}}^{\widetilde{\mathfrak{g}} l_{\ell}}$ (since the actions of $\mathfrak{H}$ and $\widehat{\mathfrak{s l}}_{\ell}$ commute). Therefore it is enough to prove that under (6.34) we have

$$
\left[\mathcal{O}\left(\Gamma_{n}\right)\right]_{\bullet, j}=V_{\omega_{0}}^{\widetilde{\mathfrak{g}}}\left[\xi_{\gamma}^{-1}\left(\omega_{0}\right)-n \delta, j\right] .
$$

Recall that by definition $\left[\mathcal{O}\left(\Gamma_{n}\right)\right]_{\bullet, j}$ is the eigenspace of eigenvalue $j$ for the action of the Casimir operator $\partial$ associated with the $\mathfrak{H}$-action of level $m \ell$ on $[\mathcal{O}(\Gamma)]$. By the discussion in Section 6.5. under (6.34) this action is sent to the action of the $m$-th Casimir operator (6.21) associated with the $\mathfrak{H}$-action on $V_{\omega_{0}}^{\widetilde{\mathfrak{g}}_{\ell}}$ of level $\ell$. So the equation (6.36) follows from the definition of $V_{\omega_{0}}^{\widetilde{\mathfrak{g l}} \ell}\left[\xi_{\gamma}^{-1}\left(\omega_{0}\right)-n \delta, j\right]$.

Step 5. Next, consider the case $i=0$. Let $\Theta_{n, 0}$ be the image of

$$
\bigoplus_{\tilde{\mu}} V_{\tilde{\mu}}^{\tilde{\mathfrak{a}}}\left[\omega_{0}-n \delta, j\right] \otimes \operatorname{Hom}_{\tilde{\mathfrak{a}}}\left(V_{\tilde{\mu}}^{\tilde{\mathfrak{a}}}, V_{\omega_{0}}^{\tilde{\mathfrak{g}} \ell}\right)
$$

under the canonical maps $V_{\tilde{\tilde{a}}}^{\tilde{\mathfrak{a}}} \otimes \operatorname{Hom}_{\tilde{\mathfrak{a}}}\left(V_{\tilde{\mu}}^{\tilde{\mathfrak{a}}}, V_{\omega_{0}}^{\tilde{\mathfrak{g} l_{\ell}}}\right) \rightarrow V_{\omega_{0}}^{\tilde{\mathfrak{g} l}}$. Here $\tilde{\mu}$ runs over the set of all weights in $P_{+}^{\tilde{a}}$ with $\langle\tilde{\mu}, \tilde{\mu}\rangle=0$. In this step we prove that the image of $\left[\mathcal{O}\left(\Gamma_{n}\right)\right]_{0, j}$ by (6.34) is isomorphic to $\Theta_{n, 0}$ as a vector space. To do that, observe first that by the definition of $\left[\mathcal{O}\left(\Gamma_{n}\right)\right]_{0, j}$ the map (6.34) takes $\left[\mathcal{O}\left(\Gamma_{n}\right)\right]_{0, j}$ onto the subspace

$$
V_{\omega_{0}}^{\widetilde{\mathfrak{g} l_{\ell}}}\left[\xi_{\gamma}^{-1}\left(\omega_{0}\right)-n \delta\right] \cap \bigoplus_{\lambda \in A(\ell, m)_{0}} v_{\hat{\gamma}\left(\lambda^{\dagger}, \ell\right)} \otimes V_{m \ell}^{\mathfrak{H}}[j] \otimes V_{\hat{\gamma}(\lambda, m)}^{\widehat{\mathfrak{s} \mathfrak{l}_{\ell}}} .
$$

Note that $v_{\hat{\gamma}\left(\lambda^{\dagger}, \ell\right)} \otimes V_{m \ell}^{\mathfrak{H} \ell}[j] \otimes V_{\hat{\gamma}(\lambda, m)}^{\widehat{\mathfrak{s l}} l_{\ell}}$ is the submodule of $\mathcal{F}_{m, \ell}^{(0)}=V_{\omega_{0}}^{\widehat{\mathfrak{g} l} \ell}$ generated by the vector $|0, \lambda\rangle$ for the level $m$ action of $\widehat{\mathfrak{g l}}_{\ell}$. Note also that $\tilde{\mathfrak{a}}_{\omega_{0}} \simeq \widetilde{\mathfrak{g l}}_{\ell}$ by Remark 6.9. Finally, the set of weights of $V_{\omega_{0}}^{\widehat{\mathfrak{g} l}}$ is

$$
\mathrm{Wt}\left(V_{\omega_{0}}^{\widehat{\mathfrak{g l}}_{\ell}}\right)=\left\{\omega_{0}+\beta: \beta \in Q^{\mathfrak{s l} \mathfrak{l}_{\ell}}\right\},
$$

see Section 4.3, and we have the following lemma.

Lemma 6.12. (a) We have $\nu \in P_{+}^{\hat{\mathfrak{a}}_{\omega_{0}}}$ if and only if $\nu^{\prime} \in P_{+}^{\widehat{\mathfrak{g} l_{\ell}}}$.

(b) We have $\left\{\nu^{\prime}: \nu \in P_{+}^{\hat{\mathfrak{a}}_{\omega_{0}}} \cap \mathrm{Wt}\left(V_{\omega_{0}}^{\widehat{\mathfrak{g}} \ell}\right)\right\}=\left\{\hat{\gamma}(\lambda, m): \lambda \in A(\ell, m)_{0}\right\}$.

Thus, by Lemmas 6.11, 6.12 the subspace 6.38) of $V_{\omega_{0}}^{\tilde{\mathfrak{g}}_{\ell}}\left[\xi_{\gamma}^{-1}\left(\omega_{0}\right)-n \delta\right]$ is indeed equal to

$$
\bigoplus_{\tilde{\nu}} V_{\tilde{\nu}}^{\tilde{\tilde{a}} \omega_{0}}\left[\xi_{\gamma}^{-1}\left(\omega_{0}\right)-n \delta, j\right]
$$


where the sum is over all extremal weights $\tilde{\nu}$ in $P_{+}^{\tilde{\mathfrak{a}}_{\omega_{0}}} \cap \operatorname{Wt}\left(V_{\omega_{0}}^{\tilde{\mathfrak{g}} l_{\ell}}\right)$ and $V_{\tilde{\nu}}^{\tilde{\mathfrak{a}}_{\omega_{0}}}$ is identified with the $\tilde{\mathfrak{a}}_{\omega_{0}}$-submodule of $V_{\omega_{0}}^{\tilde{\mathfrak{g}}_{\ell}}$ generated by a nonzero extremal weight vector of weight $\tilde{\nu}$. Now, let us consider the space $\Theta_{n, 0}$. Recall that $\langle\tilde{\mu}, \tilde{\mu}\rangle=0$ if and only if $\tilde{\mu}$ is an extremal weight of $V_{\omega_{0}}^{\tilde{\mathfrak{g}}_{\ell}}$. Further the weight subspace corresponding to an extremal weight is one-dimensional; see Section 4.3. Thus $\Theta_{n, 0}$ is equal to the sum

$$
\bigoplus_{\tilde{\mu}} V_{\tilde{\mu}}^{\tilde{a}}\left[\omega_{0}-n \delta, j\right]
$$

where $\tilde{\mu}$ runs over the set of all extremal weights such that $V_{\omega_{0}}^{\tilde{\mathfrak{g}} l_{\ell}}$ contains an $\tilde{\mathfrak{a}}$ primitive vector of weight $\tilde{\mu}$. For such a weight $\tilde{\mu}$, let $v_{\tilde{\mu}}$ be an $\tilde{\mathfrak{a}}$-primitive vector of weight $\tilde{\mu}$. Then $V_{\tilde{\tilde{a}}}^{\tilde{\mathfrak{a}}}$ is identified with the $\tilde{\mathfrak{a}}$-submodule of $V_{\omega_{0}}^{\tilde{\mathfrak{g}} l_{\ell}}$ generated by $v_{\tilde{\mu}}$. Now, recall from (6.28) that

$$
\tilde{\mathfrak{a}}=\operatorname{ad}(\gamma)^{-1}\left(\tilde{\mathfrak{a}}_{\omega_{0}}\right) .
$$

Thus, the definition of $\Delta_{+}^{\hat{\mathfrak{a}}}$ in Section 6.3 yields

$$
\xi_{\gamma}(\tilde{\mu}) \in P_{+}^{\tilde{\mathfrak{a}}} \Longleftrightarrow \tilde{\mu} \in P_{+}^{\tilde{\mathfrak{a}}_{\omega_{0}}} .
$$

Thus the $\gamma$-action yields a linear automorphism of $V_{\omega_{0}}^{\widetilde{\mathfrak{g} l}}$ such that

$$
\gamma^{-1}\left(V_{\tilde{\mu}}^{\tilde{\mathfrak{a}}_{\omega_{0}}}\left[\xi_{\gamma}^{-1}\left(\omega_{0}\right)-n \delta, j\right]\right)=V_{\xi_{\gamma}(\tilde{\mu})}^{\tilde{\mathfrak{a}}}\left[\omega_{0}-n \delta, j\right], \quad \forall \tilde{\mu} \in P_{+}^{\tilde{\mathfrak{a}}_{\omega_{0}}} .
$$

Thus (6.40) is equal to $\Theta_{n, 0}$ by the following lemma.

Lemma 6.13. For all weights $\mu$ in $P_{+}^{\hat{\mathfrak{a}}_{\omega_{0}}} \cap \mathrm{Wt}\left(V_{\omega_{0}}^{\widehat{\mathfrak{g}_{\ell}}}\right)$ the module $V_{\omega_{0}}^{\widetilde{\mathfrak{g} l}} c_{\ell}$ contains an $\tilde{\mathfrak{a}}_{\omega_{0}}$-primitive vector of weight $\tilde{\mu}$, where $\tilde{\mu}$ is the unique extremal weight in $P^{\tilde{\mathfrak{g}} l_{\ell}}$ associated with $\mu$.

Step 6. Now we prove the general case. Fix the integers $n, j$. Let $\Theta_{n, i}$ be the image of

$$
\bigoplus_{\tilde{\nu}} V_{\tilde{\nu}}^{\tilde{\mathfrak{a}}}\left[\omega_{0}-n \delta, j\right] \otimes \operatorname{Hom}_{\tilde{\mathfrak{a}}}\left(V_{\tilde{\nu}}^{\tilde{\mathfrak{a}}}, V_{\omega_{0}}^{\widetilde{\mathfrak{g} l_{\ell}}}\right)
$$

under the canonical maps $V_{\tilde{\nu}}^{\tilde{\mathfrak{a}}} \otimes \operatorname{Hom}_{\tilde{\mathfrak{a}}}\left(V_{\tilde{\nu}}^{\tilde{\mathfrak{a}}}, V_{\omega_{0}}^{\tilde{\mathfrak{g} l_{\ell}}}\right) \rightarrow V_{\omega_{0}}^{\tilde{\mathfrak{g} l_{\ell}}}$. Here the sum is over all weights $\tilde{\nu} \in P_{+}^{\tilde{a}}$ such that $\langle\tilde{\nu}, \tilde{\nu}\rangle=-2 i$. On the other hand, let $\Theta_{n, i}^{\prime}$ be the image of

$$
\bigoplus_{\tilde{\mu}} V_{\tilde{\mu}}^{\tilde{\mathfrak{a}}_{\omega_{0}}}\left[\xi_{\gamma}^{-1}\left(\omega_{0}\right)-n \delta, j\right] \otimes \operatorname{Hom}_{\tilde{\mathfrak{a}}_{\omega_{0}}}\left(V_{\tilde{\mu}}^{\tilde{\mathfrak{a}}_{\omega_{0}}}, V_{\omega_{0}}^{\widetilde{\mathfrak{g} l_{\ell}}}\right)
$$

under the canonical map $V_{\tilde{\mu}}^{\tilde{\mathfrak{a}}_{\omega_{0}}} \otimes \operatorname{Hom}_{\tilde{\mathfrak{a}}_{\omega_{0}}}\left(V_{\tilde{\mu}}^{\tilde{\mathfrak{a}}_{\omega}}, V_{\omega_{0}}^{\tilde{\mathfrak{g} l_{\ell}}}\right) \rightarrow V_{\omega_{0}}^{\tilde{\mathfrak{g}}_{\ell}}$, where the sum is over all weights $\tilde{\mu} \in P_{+}^{\tilde{\mathfrak{a}}_{\omega}}$ such that $\langle\tilde{\mu}, \tilde{\mu}\rangle=-2 i$. Then the same argument as in Step 5 implies that

$$
\Theta_{n, i}=\gamma^{-1}\left(\Theta_{n, i}^{\prime}\right)
$$

since the composition by the automorphism $\gamma^{-1}$ of $V_{\omega_{0}}^{\tilde{\mathfrak{g}}_{\ell}}$ yields a linear isomorphism

$$
\operatorname{Hom}_{\tilde{\mathfrak{a}}}\left(V_{\xi_{\gamma}(\tilde{\mu})}^{\tilde{\mathfrak{a}}}, V_{\omega_{0}}^{\widetilde{\mathfrak{g} l_{\ell}}}\right)=\operatorname{Hom}_{\tilde{\mathfrak{a}}_{\omega_{0}}}\left(V_{\tilde{\mu}}^{\tilde{\mathfrak{a}}_{\omega_{0}}}, V_{\omega_{0}}^{\widetilde{\mathfrak{g} l} l}\right) .
$$


Let us prove that (6.34) maps $\left[\mathcal{O}\left(\Gamma_{n}\right)\right]_{i, j}$ onto $\Theta_{n, i}^{\prime}$. The proof of Step 5 implies that (6.34) maps $\left[\mathcal{O}\left(\Gamma_{n}\right)\right]_{0, j}$ onto $\Theta_{n, 0}^{\prime}$. By (6.10) we have

$$
U^{-}\left(\widehat{\mathfrak{s l}}_{m}\right)_{i}\left(\left[\mathcal{O}\left(\Gamma_{n}\right)\right]_{0, j}\right)=\left[\mathcal{O}\left(\Gamma_{n}\right)\right]_{i, j}
$$

By (6.25) we also have

$$
U^{-}\left(\widehat{\mathfrak{s l}}_{m}\right)_{i}\left(\Theta_{n, 0}^{\prime}\right) \subset \Theta_{n, i}^{\prime},
$$

because the actions of $\widehat{\mathfrak{s l}}_{m}$ and $\hat{\mathfrak{a}}_{\omega_{0}}$ commute with each other. Therefore, we have

$$
\left[\mathcal{O}\left(\Gamma_{n}\right)\right]_{i, j} \subset \Theta_{n, i}^{\prime} \text {. }
$$

On the other hand, Step 4 implies that

$$
\left[\mathcal{O}\left(\Gamma_{n}\right)\right]_{\bullet, j}=\bigoplus_{i \geqslant 0} \Theta_{n, i}^{\prime}
$$

Thus we have the equality $\left[\mathcal{O}\left(\Gamma_{n}\right)\right]_{i, j}=\Theta_{n, i}^{\prime}$. The theorem is proved.

Proof of Lemma 6.11. A direct computation shows that

$$
\frac{1}{2}\langle\gamma, \gamma\rangle=\frac{1}{2} \sum_{p=1}^{\ell} s_{p}^{2} .
$$

Now, consider the partition $\lambda^{c}=\left(\lambda_{1}, \ldots, \lambda_{k \ell}\right)$. We choose $k$ to be large enough such that $\lambda_{k \ell}=0$. Write

$$
\begin{gathered}
\lambda_{i}-i+1=\left(a_{i}-1\right) \ell+b_{i}, \quad 1 \leqslant b_{i} \leqslant \ell, \\
i-1=a_{i}^{\prime} \ell+b_{i}^{\prime}, \quad 0 \leqslant b_{i}^{\prime} \leqslant \ell-1 .
\end{gathered}
$$

The number of 0 -nodes in the $i$-th row of the Young diagram associated with $\lambda^{c}$ is equal to $a_{i}+a_{i}^{\prime}$. So, by definition of $n_{0}\left(\lambda^{c}\right)$, we have

$$
n_{0}\left(\lambda^{c}\right)=\sum_{i=1}^{k \ell}\left(a_{i}+a_{i}^{\prime}\right) .
$$

We have

$$
\sum_{i=1}^{k \ell} a_{i}^{\prime}=\frac{-k(-k+1) \ell}{2}
$$

By the definition of the bijection $\tau$ in Section [6.5, see also [25, sec. 2.1], we have

$$
\begin{aligned}
\sum_{i=1}^{k \ell} a_{i} & =\sum_{p=1}^{\ell}\left((-k+1)+(-k+2)+\cdots+s_{p}\right) \\
& =\frac{1}{2} \sum_{p=1}^{\ell} s_{p}^{2}-\frac{-k(-k+1) \ell}{2} .
\end{aligned}
$$

This proves part $(a)$. For part $(b)$, note that $(a)$ and (6.24) yield

$$
D(|0, s\rangle)=-\frac{1}{2}\langle\gamma, \gamma\rangle|0, s\rangle \text {. }
$$

Further $|0, s\rangle$ is a weight vector for the level one representation of $\widehat{\mathfrak{g l}}_{\ell}$ with the weight $\omega_{0}-\gamma$; see [35, (28)]. Thus $|0, s\rangle$ is a weight vector for the level one representation of $\tilde{\mathfrak{g l}}_{\ell}$ with the weight

$$
\xi_{\gamma}^{-1}\left(\omega_{0}\right)=\omega_{0}-\gamma-\frac{1}{2}\langle\gamma, \gamma\rangle \delta
$$


The latter is an extremal weight; see Section 4.3 .

Proof of Lemma 6.12. Part $(a)$ is easy and is left to the reader. It follows from the formula for $\tilde{\mathfrak{a}}_{\omega_{0}}$ in Remark 6.9. Next, we concentrate on part (b). By (6.13), the set of all dominant integral weights of $\widehat{\mathfrak{s l}}_{\ell}$ of level $m$ is

$$
\begin{aligned}
\{\hat{\gamma}(\lambda, m): \lambda \in A(\ell, m)\} & =\left\{\left(m-\lambda_{1}+\lambda_{\ell}\right) \omega_{0}+\sum_{p=1}^{\ell-1}\left(\lambda_{p}-\lambda_{p+1}\right) \omega_{p}: \lambda \in A(\ell, m)\right\} \\
& =\left\{m \omega_{0}+\sum_{p=1}^{\ell-1}\left(\lambda_{p}-\lambda_{p+1}\right)\left(\omega_{p}-\omega_{0}\right): \lambda \in A(\ell, m)\right\} .
\end{aligned}
$$

Set $\beta=\sum_{p=1}^{\ell-1}\left(\lambda_{p}-\lambda_{p+1}\right)\left(\omega_{p}-\omega_{0}\right)$ with $\lambda \in A(\ell, m)$. Identifying $\omega_{p}-\omega_{0}$ with the $\ell$-tuple (6.29), a short computation shows that $\beta \in Q^{\mathfrak{s l}_{\ell}}$ if and only if $\lambda$ belongs to the subset $A(\ell, m)_{0}$ of $A(\ell, m)$. Therefore, by (6.39), we have

$$
\left\{\hat{\gamma}(\lambda, m): \lambda \in A(\ell, m)_{0}\right\}=\left\{\nu^{\prime}: \nu \in \mathrm{Wt}\left(V_{\omega_{0}}^{\widehat{\mathfrak{g} l} \ell}\right), \nu^{\prime} \in P_{+}^{\widehat{\mathfrak{g}} l_{\ell}}\right\} .
$$

Thus, part (b) follows from part $(a)$.

Proof of Lemma 6.13. Fix a weight $\mu$ in $P_{+}^{\hat{\mathfrak{a}}_{\omega}} \cap \mathrm{Wt}\left(V_{\omega_{0}}^{\widehat{\mathfrak{g}}_{\ell}}\right)$. Recall that

$$
\tilde{\mu}=\mu-\frac{1}{2}\langle\mu, \mu\rangle \delta
$$

Fix a nonzero element $v \in V_{\omega_{0}}^{\tilde{\mathfrak{g}}_{\ell}}$ of weight $\tilde{\mu}$. We must prove that $v$ is $\tilde{\mathfrak{a}}_{\omega_{0}}$-primitive. The argument is taken from [11, sec. 6.2]. By Remark 6.9, it is enough to prove that $\tilde{\mu}+\nu$ is not a weight of $V_{\omega_{0}}^{\tilde{\mathfrak{g}}_{\ell}}$ for any element $\nu$ in the set

$$
\left\{\alpha_{p, p+1}, \tilde{\mu}-\alpha_{1, \ell}+m \delta: p=1,2, \ldots, \ell-1\right\} .
$$

In fact, since $\tilde{\mu} \in P_{+}^{\tilde{a}}$, for such a $\nu$ we have

$$
\langle\tilde{\mu}+\nu, \tilde{\mu}+\nu\rangle=\langle\nu, \nu\rangle+2\langle\tilde{\mu}, \nu\rangle=2+2\langle\tilde{\mu}, \nu\rangle>0 .
$$

Therefore $\tilde{\mu}+\nu$ is not a weight of $V_{\omega_{0}}^{\tilde{\mathfrak{g}}_{\ell}}$ by Section 4.3.

Remark 6.14. Now, assume that the $\ell$-charge $s$ has any weight $d$, i.e., that we have $\sum_{p} s_{p}=d$, where $d$ may be nonzero. Thus $\gamma$ belongs to $P^{\mathfrak{s l}_{\ell}}$ but not necessarily to $Q^{\mathfrak{s l}_{\ell}}$. So it defines an element $\xi_{\gamma}$ in the extended affine Weyl group

$$
\widehat{\mathfrak{S}}_{\ell}^{e t}=\mathfrak{S}_{\ell} \ltimes P^{\mathfrak{s l}_{\ell}} \text {. }
$$

Thus it acts on $P^{\widehat{\mathfrak{s} l} \ell}$ as in (4.4) and we have again

$$
\hat{\gamma}(s, m)=\xi_{\gamma}^{-1}\left(\omega_{0}\right)^{\prime} .
$$

Thus, by (4.17), (6.18) and (6.19) we have

$$
\mathcal{F}_{m, \ell}^{(s)}=V_{\omega_{d \text { mod } \ell}}^{\widehat{\mathfrak{g l}}_{\ell}}\left[\xi_{\gamma}^{-1}\left(\omega_{0}\right)\right] .
$$

Now, the left-hand side is identified with $[\mathcal{O}(\Gamma)]$ by (5.20). Further, the extended affine Weyl group is isomorphic to the semi-direct product $\Omega \ltimes \widehat{\mathfrak{S}}_{\ell}$, where $\Omega=$ 
$P^{\mathfrak{s l}_{\ell}} / Q^{\mathfrak{s l}_{\ell}}$ is the group of automorphisms of the Dynkin diagram of $\widehat{\mathfrak{s l}}_{\ell}$; see, e.g., 5 , (3.1.6)]. Its braid group acts on the sum

$$
\bigoplus_{d=0}^{\ell-1} V_{\tilde{\omega}_{d}}^{\tilde{\mathfrak{g}}_{\ell}}, \quad \tilde{\omega}_{d}=\omega_{d \bmod \ell}-\frac{1}{2}\left\langle\omega_{d \bmod \ell}, \omega_{d \bmod \ell}\right\rangle=\xi_{\omega_{d \bmod \ell}-\omega_{0}}\left(\omega_{0}\right),
$$

such that the action of the braid group of $\widehat{\mathfrak{S}}_{\ell}$ preserves each summand. A direct computation using (6.29) shows that $\gamma \in \omega_{d \bmod \ell}-\omega_{0}+Q^{\mathfrak{s} l_{\ell}}$. Thus, for each integer $n \geqslant 0$ we get an isomorphism

$$
V_{\tilde{\omega}_{d}}^{\widetilde{\mathfrak{g}}_{\ell}}\left[\xi_{\gamma}^{-1}\left(\omega_{0}\right)-n \delta\right]=V_{\omega_{0}}^{\widetilde{\mathfrak{g} l}}\left[\omega_{0}-n \delta\right] .
$$

So using (6.18) we get an isomorphism

$$
\mathcal{F}_{m, \ell}^{(d)}=V_{\omega_{d \bmod \ell}}^{\tilde{\mathfrak{g}}_{\ell}}=V_{\tilde{\omega}_{d}}^{\tilde{\mathfrak{g}}_{\ell}},
$$

where the second equality is given by a shift on the weight. Now, Lemma 6.11 has the following analogue. The weight of $|0, s\rangle$, regarded as an element in $V_{\omega_{d \bmod \ell}}^{\widetilde{\mathfrak{g l}}_{\ell}}$, is

$$
\xi_{\gamma}^{-1}\left(\omega_{0}\right)+\frac{1}{2}\left\langle\omega_{d \bmod \ell}, \omega_{d \bmod \ell}\right\rangle \delta
$$

Thus its weight in $V_{\tilde{\omega}_{d}}^{\widetilde{\mathfrak{g} l_{\ell}}}$ is $\xi_{\gamma}^{-1}\left(\omega_{0}\right)$. Using the same argument as in Step 2 and (6.42) this implies that

$$
\left[\mathcal{O}\left(\Gamma_{n}\right)\right]=V_{\omega_{0}}^{\widetilde{\mathfrak{g}} \mathfrak{l}_{\ell}}\left[\omega_{0}-n \delta\right] .
$$

We can use this isomorphism to identify the filtration on the left-hand side in the same way as above. For example, note that (6.42) identifies

$$
\bigoplus_{\tilde{\mu} \in P_{+}^{\tilde{\tilde{a}} \omega_{0}} ;\langle\tilde{\mu}, \tilde{\mu}\rangle=-2 i} V_{\tilde{\mu}}^{\tilde{\mathfrak{a}}_{0}}\left[\xi_{\gamma}^{-1}\left(\omega_{0}\right)-n \delta, j\right] \otimes \operatorname{Hom}_{\tilde{\mathfrak{a}}_{\omega_{0}}}\left(V_{\tilde{\mu}}^{\tilde{\mathfrak{a}}_{\omega_{0}}}, V_{\tilde{\omega}_{d}}^{\tilde{\mathfrak{g} l_{\ell}}}\right)
$$

with

$$
\bigoplus_{\tilde{\nu} \in P_{+}^{\tilde{a}} ;\langle\tilde{\nu}, \tilde{\nu}\rangle=-2 i} V_{\tilde{\nu}}^{\tilde{\mathfrak{a}}}\left[\omega_{0}-n \delta, j\right] \otimes \operatorname{Hom}_{\tilde{\mathfrak{a}}}\left(V_{\tilde{\nu}}^{\tilde{\mathfrak{a}}}, V_{\omega_{0}}^{\tilde{\mathfrak{g} l} \ell}\right) .
$$

The same proof as in Lemma 6.12 (b) yields that

$$
\left\{\nu^{\prime}: \nu \in P_{+}^{\hat{\mathfrak{a}}_{\omega_{0}}} \cap \mathrm{Wt}\left(V_{\omega_{d \bmod \ell}}^{\widehat{\mathfrak{g l}}_{\ell}}\right)\right\}=\left\{\hat{\gamma}(\lambda, m): \lambda \in A(\ell, m)_{d}\right\} .
$$

Further, under the action of $\omega_{d \bmod \ell}-\omega_{0}$, viewed as an element of the braid group of $\widehat{\mathfrak{S}}_{\ell}^{e t}$, Lemma 6.13 implies that the set $P_{+}^{\hat{\mathfrak{a}}_{0}} \cap \mathrm{Wt}\left(V_{\omega_{d \bmod \ell}}^{\widehat{\mathfrak{g}}_{\ell}}\right)$ is in bijection with the set of highest weights of the $\tilde{\mathfrak{a}}_{\omega_{0}}$-submodules in $V_{\tilde{\omega}_{d}}^{\tilde{\mathfrak{g}}_{\ell}}$. The details are left to the reader.

\section{Appendix A. Reminder on Hecke algebras}

A.1. Affine Hecke algebras. The affine Hecke algebra of type $G L_{n}$ with parameter $\zeta \in \mathbb{C}^{\times}$is the $\mathbb{C}$-algebra $\hat{\mathbf{H}}_{\zeta}(n)$ generated by the symbols $X_{1}, X_{2}, \ldots, X_{n}$, 
$T_{1}, T_{2}, \ldots, T_{n-1}$ modulo the defining relations

$$
\begin{gathered}
X_{i} X_{j}=X_{j} X_{i}, \quad 1 \leqslant i, j \leqslant n, \\
T_{i} X_{j}=X_{j} T_{i}, \quad j \neq i, i+1, \\
T_{i} X_{i} T_{i}=\zeta X_{i+1}, \quad 1 \leqslant i \leqslant n-1, \\
\left(T_{i}+1\right)\left(T_{i}-\zeta\right)=0, \quad 1 \leqslant i \leqslant n-1, \\
T_{i} T_{j}=T_{j} T_{i}, \quad|i-j|>2, \\
T_{i} T_{i+1} T_{i}=T_{i+1} T_{i} T_{i+1}, \quad 1 \leqslant i \leqslant n-2 .
\end{gathered}
$$

For $I \subset\{1,2, \ldots, n-1\}$ let $\hat{\mathbf{H}}_{\zeta}(I) \subset \hat{\mathbf{H}}_{\zeta}(n)$ be the corresponding parabolic subalgebra. It is generated by the elements $T_{i}, X_{j}$ with $i \in I, j=1,2, \ldots, n$. For a reduced expression $w=s_{i_{1}} s_{i_{2}} \cdots s_{i_{k}}$ of an element $w \in \mathfrak{S}_{n}$ we write $T_{w}=T_{i_{1}} T_{i_{2}} \cdots T_{i_{k}}$. We abbreviate $T_{i j}=T_{s_{i j}}$. Let $D_{I}$ be the set of minimal length representatives of the left cosets in $\mathfrak{S}_{n} / \mathfrak{S}_{I}$. We'll abbreviate $D_{I, J}=D_{I}^{-1} \cap D_{J}$. For $x \in D_{I, J}$ the map

$$
\mathfrak{S}_{I \cap x J} \rightarrow \mathfrak{S}_{x^{-1} I \cap J}, \quad w \mapsto x^{-1} w x
$$

defines a length-preserving homomorphism. Hence there is a $\mathbb{C}$-algebra isomorphism

$$
\hat{\mathbf{H}}_{\zeta}(I \cap x J) \rightarrow \hat{\mathbf{H}}_{\zeta}\left(x^{-1} I \cap J\right), \quad T_{w} \mapsto T_{x^{-1} w x}, \quad X_{j} \mapsto X_{x^{-1}(j)} .
$$

Let

$$
\operatorname{Rep}\left(\hat{\mathbf{H}}_{\zeta}\left(x^{-1} I \cap J\right)\right) \rightarrow \operatorname{Rep}\left(\hat{\mathbf{H}}_{\zeta}(I \cap x J)\right), \quad M \mapsto{ }^{x} M
$$

be the corresponding twist functor. The following is well known; see, e.g., [28, thm. 1].

Lemma A.1 (Affine Mackey theorem). Let $M \in \operatorname{Rep}\left(\hat{\mathbf{H}}_{\zeta}(J)\right)$. The module

$$
\operatorname{Res}_{\hat{\mathbf{H}}_{\zeta}(I)}^{\hat{\hat{H}}_{\zeta}(n)} \operatorname{Ind}_{\hat{\mathbf{H}}_{\zeta}(J)}^{\hat{\mathbf{H}}_{\zeta}(n)}(M)
$$

admits a filtration with subquotients isomorphic to

$$
\operatorname{Ind}_{\hat{\mathbf{H}}_{\zeta}(I \cap x J)}^{\hat{\mathbf{H}}_{\zeta}(I)} \stackrel{x}{\operatorname{Res}} \hat{\mathbf{H}}_{\hat{\mathbf{H}}_{\zeta}(J)}^{\hat{y}_{\left(x^{-1} I \cap J\right)}}(M),
$$

one for each $x \in D_{I, J}$. The subquotients are taken in any order refining the Bruhat order on $D_{I, J}$. In particular we have the inclusion

$$
\operatorname{Ind}_{\hat{\mathbf{H}}_{\zeta}(I \cap J)}^{\hat{\mathbf{H}}_{\zeta}(I)} \operatorname{Res}_{\hat{\mathbf{H}}_{\zeta}(I \cap J)}^{\hat{\mathbf{H}}_{\zeta}(J)}(M) \subset \operatorname{Res}_{\hat{\mathbf{H}}_{\zeta}(I)}^{\hat{\mathbf{H}}_{\zeta}(n)} \operatorname{Ind}_{\hat{\mathbf{H}}_{\zeta}(J)}^{\hat{\mathbf{H}}_{\zeta}(n)}(M) .
$$

A.2. Cyclotomic Hecke algebras. The cyclotomic Hecke algebra $\mathbf{H}_{\zeta}(n, \ell)$ associated with $\Gamma_{n}$ and the parameters $\zeta, v_{1}, v_{2}, \ldots, v_{\ell} \in \mathbb{C}^{\times}$is the quotient of $\hat{\mathbf{H}}_{\zeta}(n)$ by the two-sided ideal generated by the element

$$
\left(X_{1}-v_{1}\right)\left(X_{1}-v_{2}\right) \ldots\left(X_{1}-v_{\ell}\right) \text {. }
$$

We'll denote the image of the generator $X_{1}$ in $\mathbf{H}_{\zeta}(n, \ell)$ by the symbol $T_{0}$. For a subset $I \subset\{0,1, \ldots, n-1\}$ we define $\Gamma_{I} \subset \Gamma_{n}$ as the subgroup $\mathfrak{S}_{I}$ if $0 \notin I$, or as the subgroup generated by $\mathfrak{S}_{I \backslash\{0\}}$ and $\left\{\gamma_{1} ; \gamma \in \Gamma\right\}$ otherwise. This yields all parabolic subgroups of $\Gamma_{n}$. We consider also the parabolic subalgebra $\mathbf{H}_{\zeta}(I, \ell) \subset \mathbf{H}_{\zeta}(n, \ell)$ which is the subalgebra generated by the elements $T_{i}$ with $i \in I$. Recall that $T_{0}=X_{1}$. To unburden the notation, we abbreviate

$$
\mathbf{H}\left(\Gamma_{n}\right)=\mathbf{H}_{\zeta}(n, \ell), \quad \mathbf{H}\left(\mathfrak{S}_{m}\right)=\mathbf{H}_{\zeta}(m), \quad \mathbf{H}\left(\Gamma_{I}\right)=\mathbf{H}_{\zeta}(I, \ell) .
$$


For $I=\{0,1, \ldots, n+m\} \backslash\{n\}$ we also write

$$
\mathbf{H}\left(\Gamma_{n, m}\right)=\mathbf{H}\left(\Gamma_{I}\right) \subset \mathbf{H}\left(\Gamma_{n+m}\right) .
$$

Note that

$$
\mathbf{H}\left(\Gamma_{n, m}\right) \simeq \mathbf{H}\left(\Gamma_{n}\right) \otimes \mathbf{H}\left(\mathfrak{S}_{m}\right) .
$$

A.3. Induction/restriction for cyclotomic Hecke algebras. We'll abbreviate

$$
\begin{aligned}
& { }^{\mathbf{H}} \operatorname{Ind}_{n}=\operatorname{Ind}_{\mathbf{H}\left(\Gamma_{n-1}\right)}^{\mathbf{H}\left(\Gamma_{n}\right)}, \quad \mathbf{H}_{\operatorname{Res}_{n}}=\operatorname{Res}_{\mathbf{H}\left(\Gamma_{n-1}\right)}^{\mathbf{H}\left(\Gamma_{n}\right)}, \\
& { }^{\mathbf{H}_{1 n d}} \operatorname{Ind}_{n,\left(m^{r}\right)}=\operatorname{Ind}_{\mathbf{H}\left(\Gamma_{n,\left(m^{r}\right)}\right)}^{\mathbf{H}\left(\Gamma_{n+m r}\right)}, \quad \mathbf{H}_{\boldsymbol{R e s}_{n,\left(m^{r}\right)}}=\operatorname{Res}_{\mathbf{H}\left(\Gamma_{n,\left(m^{r}\right)}\right)}^{\mathbf{H}\left(\Gamma_{n+m r}\right)}, \\
& { }^{\mathbf{H}} \operatorname{Ind}_{n, m r}=\operatorname{Ind}_{\mathbf{H}\left(\Gamma_{n, m r}\right)}^{\mathbf{H}\left(\Gamma_{n+m r}\right)}, \quad \mathbf{H}_{\operatorname{Res}_{n, m r}}=\operatorname{Res}_{\mathbf{H}\left(\Gamma_{n, m r}\right)}^{\mathbf{H}\left(\Gamma_{n+m r}\right)} .
\end{aligned}
$$

We also write

$$
\begin{aligned}
& { }^{\mathbf{H}} \operatorname{Ind}_{\left(m^{r}\right)}={ }^{\mathbf{H}} \operatorname{Ind}_{\mathfrak{S}_{m}^{r r}}^{\mathfrak{S}_{m r}}: \operatorname{Rep}\left(\mathbf{H}\left(\mathfrak{S}_{m}^{r}\right)\right) \rightarrow \operatorname{Rep}\left(\mathbf{H}\left(\mathfrak{S}_{m r}\right)\right), \\
& { }^{\mathbf{H}} \operatorname{Res}_{\left(m^{r}\right)}={ }^{\mathbf{H}} \operatorname{Res}_{\mathfrak{S}_{m}^{r r}}^{\mathfrak{S}_{m r}}: \operatorname{Rep}\left(\mathbf{H}\left(\mathfrak{S}_{m r}\right)\right) \rightarrow \operatorname{Rep}\left(\mathbf{H}\left(\mathfrak{S}_{m}^{r}\right)\right) .
\end{aligned}
$$

Now, we consider the Mackey decomposition of the functor

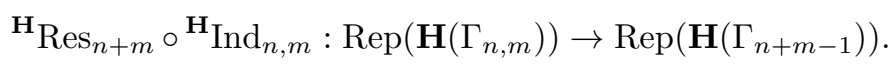

A short computation shows that a set of representatives of the double cosets in

$$
\Gamma_{n+m-1} \backslash \Gamma_{n+m} / \Gamma_{n, m}
$$

is $\left\{\gamma_{n+m}, s_{n, n+m}: \gamma \in \Gamma\right\}$. For

$$
I=\{0, \ldots, n+m-1\} \backslash\{n-1, n\}, \quad J=\{0, \ldots, n+m-2\} \backslash\{n-1\}
$$

we have

$$
\mathbf{H}\left(\Gamma_{I}\right) \subset \mathbf{H}\left(\Gamma_{n, m}\right), \quad \mathbf{H}\left(\Gamma_{J}\right)=\mathbf{H}\left(\Gamma_{n-1, m}\right) \subset \mathbf{H}\left(\Gamma_{n+m-1}\right) .
$$

Further, there is an algebra isomorphism

$$
\varphi: \mathbf{H}\left(\Gamma_{J}\right) \rightarrow \mathbf{H}\left(\Gamma_{I}\right), T_{w} \mapsto T_{s w s^{-1}}, X_{i} \mapsto X_{s i},
$$

where $s=s_{n} s_{n+1} \cdots s_{n+m-1}$. For each $i, p$ we write $X_{i}^{p}=\left(X_{i}\right)^{p}$. We have the following decomposition. It is well known in the case $m=1$; see, e.g., [21, lem. 7.6.1] in the degenerate case.

Proposition A.2. (a) We have an isomorphism of $\mathbf{H}\left(\Gamma_{n+m-1}\right)$-modules

$$
\mathbf{H}\left(\Gamma_{n+m}\right)=\bigoplus_{0 \leqslant p<\ell} \bigoplus_{1 \leqslant j \leqslant n+m} \mathbf{H}\left(\Gamma_{n+m-1}\right) T_{j, n+m} X_{j}^{p}
$$

(b) We have an isomorphism of $\left(\mathbf{H}\left(\Gamma_{n+m-1}\right), \mathbf{H}\left(\Gamma_{n, m}\right)\right)$-bimodules

$$
\mathbf{H}\left(\Gamma_{n+m}\right)=\mathbf{H}\left(\Gamma_{n+m-1}\right) T_{n, n+m} \mathbf{H}\left(\Gamma_{n, m}\right) \oplus \bigoplus_{0 \leqslant p<\ell} \mathbf{H}\left(\Gamma_{n+m-1}\right) X_{n+m}^{p} \mathbf{H}\left(\Gamma_{n, m}\right) .
$$

(c) There are isomorphisms of $\left(\mathbf{H}\left(\Gamma_{n+m-1}\right), \mathbf{H}\left(\Gamma_{n, m}\right)\right)$-bimodules

$$
\begin{aligned}
\mathbf{H}\left(\Gamma_{n+m-1}\right) T_{n, n+m} \mathbf{H}\left(\Gamma_{n, m}\right) & =\mathbf{H}\left(\Gamma_{n+m-1}\right) \otimes_{\mathbf{H}\left(\Gamma_{n-1, m}\right)} \mathbf{H}\left(\Gamma_{n, m}\right), \\
\mathbf{H}\left(\Gamma_{n+m-1}\right) X_{n+m}^{p} \mathbf{H}\left(\Gamma_{n, m}\right) & =\mathbf{H}\left(\Gamma_{n+m-1}\right) \otimes_{\mathbf{H}\left(\Gamma_{n, m-1}\right)} \mathbf{H}\left(\Gamma_{n, m}\right),
\end{aligned}
$$

where the algebra homomorphism $\mathbf{H}\left(\Gamma_{n-1, m}\right) \rightarrow \mathbf{H}\left(\Gamma_{n, m}\right)$ is given by $\varphi$. 
Proof. Part (a) is standard; see, e.g., 21, lem. 7.6.1] in the degenerate case. Let us concentrate on $(b)$. Write $t_{j, i}=T_{j} T_{j-1} \cdots T_{i}$ for $1 \leqslant i \leqslant j$, and $t_{j, i}=1$ for $i>j$. By $(a)$ we are reduced to proving the following identities:

$$
\bigoplus_{0 \leqslant p<\ell} \bigoplus_{1 \leqslant j \leqslant n} \mathbf{H}\left(\Gamma_{n+m-1}\right) t_{n+m-1, j} X_{j}^{p}=\mathbf{H}\left(\Gamma_{n+m-1}\right) t_{n+m-1, n} \mathbf{H}\left(\Gamma_{n, m}\right),
$$

$$
\bigoplus_{0 \leqslant p<\ell} \bigoplus_{n<j \leqslant n+m} \mathbf{H}\left(\Gamma_{n+m-1}\right) t_{n+m-1, j} X_{j}^{p}=\bigoplus_{0 \leqslant p<\ell} \mathbf{H}\left(\Gamma_{n+m-1}\right) X_{n+m}^{p} \mathbf{H}\left(\Gamma_{n, m}\right) .
$$

We have

$$
u t_{n+m-1, n}=t_{n+m-1, n} \varphi(u), \quad u \in \mathbf{H}\left(\Gamma_{n-1, m}\right),
$$

because for $i=1,2, \ldots, n-1$ and $j \in J \backslash\{0\}$ we have

$$
\begin{gathered}
T_{j} t_{n+m-1, n}=t_{n+m-1, n} T_{s(j)}=t_{n+m-1, n} \varphi\left(T_{j}\right), \\
X_{i} t_{n+m-1, n}=t_{n+m-1, n} X_{i}=t_{n+m-1, n} \varphi\left(X_{i}\right) .
\end{gathered}
$$

Hence, by $(a)$, the right-hand side of (A.2) is

$$
\begin{aligned}
& =\bigoplus_{0 \leqslant p<\ell} \bigoplus_{1 \leqslant j \leqslant n} \mathbf{H}\left(\Gamma_{n+m-1}\right) t_{n+m-1, n} \mathbf{H}\left(\Gamma_{I}\right) t_{n-1, j} X_{j}^{p} \\
& =\bigoplus_{0 \leqslant p<\ell} \bigoplus_{1 \leqslant j \leqslant n} \mathbf{H}\left(\Gamma_{n+m-1}\right) \mathbf{H}\left(\Gamma_{n-1, m}\right) t_{n+m-1, n} t_{n-1, j} X_{j}^{p} \\
& =\bigoplus_{0 \leqslant p<\ell} \bigoplus_{1 \leqslant j \leqslant n} \mathbf{H}\left(\Gamma_{n+m-1}\right) t_{n+m-1, j} X_{j}^{p} .
\end{aligned}
$$

This proves (A.2). Next, a short calculation involving the relation

$$
X_{j+1}^{p} T_{j}-T_{j} X_{j}^{p} \in \mathbb{C}\left[X_{j}, X_{j+1}\right]
$$

proves that the sum

$$
\sum_{0 \leqslant p<\ell} \sum_{n<j \leqslant n+m} \mathbf{H}\left(\Gamma_{n+m-1}\right) t_{n+m-1, j} X_{j}^{p}
$$

is indeed a direct sum; i.e., it is equal to the left-hand side of (A.3). Thus the identity (A.3) follows from the following equalities:

$$
\begin{aligned}
\mathbf{H}\left(\Gamma_{n+m-1}\right) X_{n+m}^{p} \mathbf{H}\left(\Gamma_{n, m}\right) & =\sum_{n<j \leqslant n+m} \mathbf{H}\left(\Gamma_{n+m-1}\right) X_{n+m}^{p} T_{j, n+m} \\
& =\sum_{n<j \leqslant n+m} \mathbf{H}\left(\Gamma_{n+m-1}\right) X_{n+m}^{p} t_{n+m-1, j} \\
& =\sum_{n<j \leqslant n+m} \mathbf{H}\left(\Gamma_{n+m-1}\right) t_{n+m-1, j} X_{j}^{p} .
\end{aligned}
$$

Finally, let us prove $(c)$. To prove the second claim, note that the left multiplication by the element $X_{n+m}^{p}$, which is invertible, yields an isomorphism of $\left(\mathbf{H}\left(\Gamma_{n+m-1}\right), \mathbf{H}\left(\Gamma_{n, m}\right)\right)$-sub-bimodules of $\mathbf{H}\left(\Gamma_{n+m}\right)$ :

$$
\mathbf{H}\left(\Gamma_{n+m-1}\right) \mathbf{H}\left(\Gamma_{n, m}\right)=X_{n+m}^{p} \mathbf{H}\left(\Gamma_{n+m-1}\right) \mathbf{H}\left(\Gamma_{n, m}\right)=\mathbf{H}\left(\Gamma_{n+m-1}\right) X_{n+m}^{p} \mathbf{H}\left(\Gamma_{n, m}\right) .
$$

Finally, the obvious epimorphism

$$
\phi: \mathbf{H}\left(\Gamma_{n+m-1}\right) \otimes_{\mathbf{H}\left(\Gamma_{n, m-1}\right)} \mathbf{H}\left(\Gamma_{n, m}\right) \rightarrow \mathbf{H}\left(\Gamma_{n+m-1}\right) \mathbf{H}\left(\Gamma_{n, m}\right)
$$


is also invertible. The reason is the following: consider the canonical isomorphisms

$$
\mathbf{H}\left(\Gamma_{n, m}\right) \simeq \mathbf{H}\left(\Gamma_{n}\right) \otimes \mathbf{H}\left(\mathfrak{S}_{m}\right), \quad \mathbf{H}\left(\Gamma_{n, m-1}\right) \simeq \mathbf{H}\left(\Gamma_{n}\right) \otimes \mathbf{H}\left(\mathfrak{S}_{m-1}\right) .
$$

By applying part $(a)$ to $\mathbf{H}\left(\mathfrak{S}_{m}\right)$ we see that

$$
\mathbf{H}\left(\Gamma_{n, m}\right)=\bigoplus_{j=n+1}^{n+m} \mathbf{H}\left(\Gamma_{n, m-1}\right) T_{j, n+m} .
$$

Therefore the left-hand side of $\phi$ is a free left $\mathbf{H}\left(\Gamma_{n+m-1}\right)$-module with basis $\{1 \otimes$ $\left.T_{j, n+m} ; n<j \leqslant n+m\right\}$, and the sum $\sum_{j=n+1}^{n+m} \mathbf{H}\left(\Gamma_{n+m-1}\right) T_{j, n+m}$ in $\mathbf{H}\left(\Gamma_{n+m}\right)$ is direct by $(a)$ again. So $\phi$ is also injective. To prove the first claim in $(c)$ we define a map

$$
\begin{aligned}
\mathbf{H}\left(\Gamma_{n+m-1}\right) \times \mathbf{H}\left(\Gamma_{n, m}\right) & \rightarrow \mathbf{H}\left(\Gamma_{n+m-1}\right) T_{n, n+m} \mathbf{H}\left(\Gamma_{n, m}\right), \\
(u, v) & \mapsto u t_{n+m-1, n} v .
\end{aligned}
$$

By (A.4) this map factors to a surjective homomorphism

$$
\psi: \mathbf{H}\left(\Gamma_{n+m-1}\right) \otimes_{\mathbf{H}\left(\Gamma_{n-1, m}\right)} \mathbf{H}\left(\Gamma_{n, m}\right) \rightarrow \mathbf{H}\left(\Gamma_{n+m-1}\right) T_{n, n+m} \mathbf{H}\left(\Gamma_{n, m}\right) .
$$

Since we have

$$
\mathbf{H}\left(\Gamma_{n, m}\right) \simeq \mathbf{H}\left(\Gamma_{n}\right) \otimes \mathbf{H}\left(\mathfrak{S}_{m}\right), \quad \mathbf{H}\left(\Gamma_{n-1, m}\right) \simeq \mathbf{H}\left(\Gamma_{n-1}\right) \otimes \mathbf{H}\left(\mathfrak{S}_{m}\right),
$$

by applying ( $a)$ to $\mathbf{H}\left(\Gamma_{n}\right)$ we see that the left-hand side of $\psi$ is a free $\mathbf{H}\left(\Gamma_{n+m-1}\right)$ module on the basis

$$
1 \otimes t_{n-1, j} X_{j}^{p}, \quad 1 \leqslant j \leqslant n, \quad 0 \leqslant p<\ell .
$$

But $\psi$ maps these elements to

$$
t_{n+m-1, j} X_{j}^{p}, \quad 1 \leqslant j \leqslant n, \quad 0 \leqslant p<\ell .
$$

Further, the latter are $\mathbf{H}\left(\Gamma_{n+m-1}\right)$-linearly independent in $\mathbf{H}\left(\Gamma_{n+m}\right)$ by $(a)$ again. Therefore $\psi$ is injective. We are done.

\section{Appendix B. Reminder on $\zeta$-Schur Algebras}

B.1. The quantized modified algebra. Let $v$ be a formal variable. The quantized modified algebra $\dot{\mathbf{U}}(n)$ of $\mathfrak{g l}_{n}$ is the associative $\mathbb{Q}(v)$-algebra with generators $E_{i}, F_{i}$, where $i=1, \ldots, n-1$ and $1_{\lambda}$ where $\lambda \in \mathbb{Z}^{n}$, with the defining relations [26. sec. 23]

- $1_{\lambda} 1_{\mu}=\delta_{\lambda, \mu} 1_{\lambda}$,

- $E_{i} F_{j}-F_{j} E_{i}=\delta_{i j} \sum_{\lambda}\left[\lambda_{i}-\lambda_{i+1}\right] 1_{\lambda}$,

- $E_{i} 1_{\lambda}=1_{\lambda+\alpha_{i}} E_{i}$,

- $1_{\lambda} F_{i}=F_{i} 1_{\lambda+\alpha_{i}}$,

- $E_{i} E_{j}=E_{j} E_{i}$ if $i \neq j \pm 1, E_{i}^{2} E_{j}-\left(v+v^{-1}\right) E_{i} E_{j} E_{i}+E_{j} E_{i}^{2}=0$ else,

- $F_{i} F_{j}=F_{j} F_{i}$ if $i \neq j \pm 1, F_{i}^{2} F_{j}-\left(v+v^{-1}\right) F_{i} F_{j} F_{i}+F_{j} F_{i}^{2}=0$ else.

Here $[m]$ is the usual $v$-analogue of $m$ for any $m \in \mathbb{N}$, and $\alpha_{i} \in \mathbb{Z}^{n}$ is the root $(0, \ldots, 0,1,-1,0, \ldots, 0)$ with 1 at the $i$-th spot. The comultiplication of $\dot{\mathbf{U}}(n)$ is the $\mathbb{Q}(v)$-algebra homomorphism

$$
\Delta: \dot{\mathbf{U}}(n) \rightarrow \prod_{\lambda, \lambda^{\prime}}\left(\dot{\mathbf{U}}(n) 1_{\lambda} \otimes \dot{\mathbf{U}}(n) 1_{\lambda^{\prime}}\right)
$$


given by

- $\Delta\left(1_{\lambda}\right)=\prod_{\lambda=\lambda^{\prime}+\lambda^{\prime \prime}} 1_{\lambda^{\prime}} \otimes 1_{\lambda^{\prime \prime}}$,

- $\Delta\left(E_{i} 1_{\lambda}\right)=\prod_{\lambda=\lambda^{\prime}+\lambda^{\prime \prime}}\left(E_{i} 1_{\lambda^{\prime}} \otimes 1_{\lambda^{\prime \prime}}+v^{\left(\alpha_{i}, \lambda^{\prime}\right)} 1_{\lambda^{\prime}} \otimes E_{i} 1_{\lambda^{\prime \prime}}\right)$,

- $\Delta\left(F_{i} 1_{\lambda}\right)=\prod_{\lambda=\lambda^{\prime}+\lambda^{\prime \prime}}\left(F_{i} 1_{\lambda^{\prime}} \otimes v^{-\left(\alpha_{i}, \lambda^{\prime \prime}\right)} 1_{\lambda^{\prime \prime}}+1_{\lambda^{\prime}} \otimes F_{i} 1_{\lambda^{\prime \prime}}\right)$.

Set $\mathcal{A}=\mathbb{Z}\left[v, v^{-1}\right]$. The integral quantized modified algebra is the $\mathcal{A}$-subalgebra $\dot{\mathbf{U}}_{\mathcal{A}}(n) \subset \dot{\mathbf{U}}(n)$ generated by the $1_{\lambda}$ 's and all quantum divided powers $E_{i}^{(d)}, F_{i}^{(d)}$. The comultiplication yields an $\mathcal{A}$-algebra homomorphism

$$
\dot{\mathbf{U}}_{\mathcal{A}}(n) \rightarrow \prod_{\lambda, \lambda^{\prime}}\left(\dot{\mathbf{U}}_{\mathcal{A}}(n) 1_{\lambda} \otimes \dot{\mathbf{U}}_{\mathcal{A}}(n) 1_{\lambda^{\prime}}\right)
$$

For $\epsilon \in \mathbb{C}^{\times}$we consider the $\mathbb{C}$-algebra

$$
\dot{\mathbf{U}}_{\epsilon}(n)=\dot{\mathbf{U}}_{\mathcal{A}}(n) \otimes_{\mathcal{A}} \mathbb{C}\left[v, v^{-1}\right] /(v-\epsilon) .
$$

For $V, V^{\prime} \in \operatorname{Rep}\left(\dot{\mathbf{U}}_{\epsilon}(n)\right)$ let $\mathbf{s}_{V, V^{\prime}}: V \otimes V^{\prime} \rightarrow V^{\prime} \otimes V$ be the permutation $v \otimes v^{\prime} \mapsto$ $v^{\prime} \otimes v$. An $R$-matrix is a $\mathbb{C}$-linear endomorphism $R_{V, V^{\prime}}$ of $V \otimes V^{\prime}$ such that the composed map

$$
\mathcal{R}_{V, V^{\prime}}=\mathbf{s}_{V, V^{\prime}} \circ R_{V, V^{\prime}}
$$

is an isomorphism of $\dot{\mathbf{U}}_{\epsilon}(n)$-modules $V \otimes V^{\prime} \rightarrow V^{\prime} \otimes V$. We fix an $R$-matrix $R_{V, V^{\prime}}$ as follows:

$$
\begin{gathered}
R_{V, V^{\prime}}\left(v \otimes v^{\prime}\right)=R\left(v \otimes v^{\prime}\right), \quad R=\bar{\Pi} \bar{\Theta} \\
\bar{\Pi}=\prod_{\lambda, \lambda^{\prime}} \epsilon^{-\left(\lambda, \lambda^{\prime}\right)} 1_{\lambda} \otimes 1_{\lambda^{\prime}}, \quad \bar{\Theta} \in \prod_{\lambda, \lambda^{\prime}}\left(\dot{\mathbf{U}}_{\epsilon}(n) 1_{\lambda} \otimes \dot{\mathbf{U}}_{\epsilon}(n) 1_{\lambda^{\prime}}\right) .
\end{gathered}
$$

Here, the element $\bar{\Theta}$ is defined as in [26, sec. 32]. We call $R$ the universal $R$ matrix. To avoid confusion we may write $R_{\epsilon}$ for $R$. We'll write $\mathcal{R}_{V, V^{\prime}}$ again for the braiding of right $\dot{\mathbf{U}}_{\epsilon}(n)$-modules $V, V^{\prime}$. If $\epsilon$ is a primitive $2 d$-th root of 1 , then we have $\epsilon^{d^{2}}=(-1)^{d}$. Hence the quantum Frobenius homomorphism [26, sec. 35.1] is a $\mathbb{C}$-algebra homomorphism

$$
\text { Fr }: \dot{\mathbf{U}}_{\epsilon}(n) \rightarrow \dot{\mathbf{U}}_{(-1)^{d}}(n)
$$

such that

- $\operatorname{Fr}\left(E_{i}^{(m)} 1_{\lambda}\right)=E_{i}^{(m / d)} 1_{\lambda / d}$ if $m \in d \mathbb{Z}$ and $\lambda \in d \mathbb{Z}^{n}$, and 0 otherwise,

- $\operatorname{Fr}\left(F_{i}^{(m)} 1_{\lambda}\right)=F_{i}^{(m / d)} 1_{\lambda / d}$ if $m \in d \mathbb{Z}$ and $\lambda \in d \mathbb{Z}^{n}$, and 0 otherwise.

The formulas in [26, sec. 3.1.5] imply that

$$
\Delta \circ \operatorname{Fr}=\operatorname{Fr} \circ \Delta \text {. }
$$

Proposition B.1. We have $(\operatorname{Fr} \otimes \operatorname{Fr})\left(R_{\epsilon}\right)=R_{(-1)^{d}}=\prod_{\lambda, \lambda^{\prime}}(-1)^{d\left(\lambda, \lambda^{\prime}\right)}\left(1_{\lambda} \otimes 1_{\lambda^{\prime}}\right)$.

Proof. To avoid confusion we'll write $\bar{\Theta}_{\epsilon}, \bar{\Pi}_{\epsilon}$ for $\bar{\Theta}, \bar{\Pi}$. If $n=2$ the proposition follows from the formula [26, sec. 4.1.4]. More precisely, since

$$
\bar{\Theta}_{\epsilon}=\prod_{\lambda, \lambda^{\prime}} \sum_{k \geqslant 0}(-1)^{k} \epsilon^{-k(k-1) / 2}\{k\}_{\epsilon} F^{(k)} 1_{\lambda} \otimes E^{(k)} 1_{\lambda^{\prime}}, \quad\{k\}_{\epsilon}=\prod_{i=1}^{k}\left(\epsilon^{i}-\epsilon^{-i}\right),
$$

we have the following formula:

$$
(\operatorname{Fr} \otimes \operatorname{Fr})\left(\bar{\Theta}_{\epsilon}\right)=\prod_{\lambda, \lambda^{\prime}}\left(1_{\lambda} \otimes 1_{\lambda^{\prime}}\right)
$$


Further, in $\dot{\mathbf{U}}_{(-1)^{d}}(n) \otimes \dot{\mathbf{U}}_{(-1)^{d}}(n)$ we also have

$$
(\operatorname{Fr} \otimes \operatorname{Fr})\left(\bar{\Pi}_{\epsilon}\right)=\prod_{\lambda, \lambda^{\prime}}(-1)^{d\left(\lambda, \lambda^{\prime}\right)}\left(1_{\lambda} \otimes 1_{\lambda^{\prime}}\right)
$$

and

$$
\bar{\Theta}_{(-1)^{d}}=\prod_{\lambda, \lambda^{\prime}}\left(1_{\lambda} \otimes 1_{\lambda^{\prime}}\right), \quad \bar{\Pi}_{(-1)^{d}}=\prod_{\lambda, \lambda^{\prime}}(-1)^{d\left(\lambda, \lambda^{\prime}\right)}\left(1_{\lambda} \otimes 1_{\lambda^{\prime}}\right) .
$$

This proves the formula for $n=2$. Now, let $n$ be any integer $\geqslant 2$. The braid group of $\mathfrak{S}_{n}$ acts on $\dot{\mathbf{U}}_{\epsilon}(n)$ via the operators $T_{1,1}^{\prime \prime}, T_{2,1}^{\prime \prime}, \ldots, T_{n-1,1}^{\prime \prime}$ in [26, sec. 41]. For $i=1,2, \ldots, n-1$ we set

$$
S_{i}=T_{i, 1}^{\prime \prime} \otimes T_{i, 1}^{\prime \prime}, \quad \bar{\theta}_{i, \epsilon}=\sum_{k \geqslant 0}(-1)^{k} \epsilon^{-k(k-1) / 2}\{k\}_{\epsilon} F_{i}^{(k)} \otimes E_{i}^{(k)} .
$$

For a reduced decomposition $s_{i_{1}} s_{i_{2}} \cdots s_{i_{r}}$ of the longest element in $\mathfrak{S}_{n}$, the universal R-matrix is given by the following formula (see [22, thm. 3]):

$$
\bar{\Theta}_{\epsilon}=\prod_{\lambda, \lambda^{\prime}} \bar{\theta}_{\epsilon}\left(1_{\lambda} \otimes 1_{\lambda^{\prime}}\right), \quad \bar{\theta}_{\epsilon}=S_{i_{r}}^{-1} \cdots S_{i_{3}}^{-1} S_{i_{2}}^{-1}\left(\bar{\theta}_{i_{1}, \epsilon}\right) \cdots S_{i_{r}}^{-1}\left(\bar{\theta}_{i_{r-1}, \epsilon}\right) \bar{\theta}_{i_{r}, \epsilon} .
$$

Thus (B.3) yields

$$
\bar{\Theta}_{(-1)^{d}}=\prod_{\lambda, \lambda^{\prime}}\left(1_{\lambda} \otimes 1_{\lambda^{\prime}}\right) .
$$

Since the braid group action is compatible with the quantum Frobenius homomorphism, see [26, sec. 41.1.9], by (B.1) we also have

$$
(\operatorname{Fr} \otimes \operatorname{Fr})\left(\bar{\Theta}_{\epsilon}\right)=\prod_{\lambda, \lambda^{\prime}}\left(1_{\lambda} \otimes 1_{\lambda^{\prime}}\right) .
$$

Finally, a direct computation yields

$$
(\operatorname{Fr} \otimes \operatorname{Fr})\left(\bar{\Pi}_{\epsilon}\right)=\prod_{\lambda, \lambda^{\prime}}(-1)^{d\left(\lambda, \lambda^{\prime}\right)}\left(1_{\lambda} \otimes 1_{\lambda^{\prime}}\right)=\bar{\Pi}_{(-1)^{d}} .
$$

This proves the proposition.

Remark B.2. It is proved in [26, prop. 33.2.3] that the assignment

$$
E_{i} 1_{\lambda} \mapsto(-1)^{i d\left(\lambda_{i}-\lambda_{i+1}\right)} E_{i} 1_{\lambda}, \quad F_{i} 1_{\lambda} \mapsto(-1)^{(i+1) d\left(\lambda_{i}-\lambda_{i+1}\right)+d} E_{i} 1_{\lambda}, \quad 1_{\lambda} \mapsto 1_{\lambda}
$$

yields a $\mathbb{C}$-algebra isomorphism $\dot{\mathbf{U}}_{1}(n) \rightarrow \dot{\mathbf{U}}_{(-1)^{d}}(n)$. Thus we can regard Fr as a map $\dot{\mathbf{U}}_{\epsilon}(n) \rightarrow \dot{\mathbf{U}}_{1}(n)$. Note that the isomorphism above does not commute with the comultiplication.

B.2. The $\zeta$-Schur algebra. Recall the set $\Lambda(n, m)$ from Section 3.1, The $v$-Schur algebra $\mathbf{S}(n, m)$ is the associative $\mathbb{Q}(v)$-algebra with 1 generated by $E_{i}, F_{i}$, where $i=1, \ldots, n-1$ and by $1_{\lambda}$, where $\lambda \in \Lambda(n, m)$, modulo the defining relations [10, thm. 2.4]

- $1_{\lambda} 1_{\mu}=\delta_{\lambda, \mu} 1_{\lambda}, \sum_{\lambda} 1_{\lambda}=1$,

- $E_{i} F_{j}-F_{j} E_{i}=\delta_{i j} \sum_{\lambda}\left[\lambda_{i}-\lambda_{i+1}\right] 1_{\lambda}$,

- $E_{i} 1_{\lambda}=1_{\lambda+\alpha_{i}} E_{i}$ if $\lambda+\alpha_{i} \in \Lambda(n, m), 0$ else,

- $1_{\lambda} E_{i}=E_{i} 1_{\lambda-\alpha_{i}}$ if $\lambda-\alpha_{i} \in \Lambda(n, m), 0$ else,

- $F_{i} 1_{\lambda}=1_{\lambda-\alpha_{i}} F_{i}$ if $\lambda-\alpha_{i} \in \Lambda(n, m), 0$ else, 
- $1_{\lambda} F_{i}=F_{i} 1_{\lambda+\alpha_{i}}$ if $\lambda+\alpha_{i} \in \Lambda(n, m), 0$ else,

- $E_{i} E_{j}=E_{j} E_{i}$ if $i \neq j \pm 1, E_{i}^{2} E_{j}-\left(v+v^{-1}\right) E_{i} E_{j} E_{i}+E_{j} E_{i}^{2}=0$ else,

- $F_{i} F_{j}=F_{j} F_{i}$ if $i \neq j \pm 1, F_{i}^{2} F_{j}-\left(v+v^{-1}\right) F_{i} F_{j} F_{i}+F_{j} F_{i}^{2}=0$ else.

The integral $v$-Schur algebra is the $\mathcal{A}$-subalgebra $\mathbf{S}_{\mathcal{A}}(n, m) \subset \mathbf{S}(n, m)$ generated by the $1_{\lambda}$ 's and all quantum divided powers $E_{i}^{(d)}, F_{i}^{(d)}$. In other words, we have a canonical isomorphism

$$
\mathbf{S}_{\mathcal{A}}(n, m)=1_{m} \dot{\mathbf{U}}_{\mathcal{A}}(n) 1_{m}, \quad 1_{m}=\sum_{\lambda \in \Lambda(n, m)} 1_{\lambda} .
$$

The comultiplication of $\dot{\mathbf{U}}_{\mathcal{A}}(n)$ factors through an $\mathcal{A}$-algebra homomorphism

$$
\Delta: \mathbf{S}_{\mathcal{A}}(n, m) \rightarrow \bigoplus_{m=m^{\prime}+m^{\prime \prime}} \mathbf{S}_{\mathcal{A}}\left(n, m^{\prime}\right) \otimes \mathbf{S}_{\mathcal{A}}\left(n, m^{\prime \prime}\right)
$$

For $\zeta, \epsilon \in \mathbb{C}^{\times}$with $\zeta=\epsilon^{2}$ we consider the $\mathbb{C}$-algebra

$$
\begin{aligned}
\mathbf{S}_{\zeta}(n, m) & =\mathbf{S}_{\mathcal{A}}(n, m) \otimes_{\mathcal{A}} \mathbb{C}\left[v, v^{-1}\right] /(v-\epsilon) \\
& =1_{m} \dot{\mathbf{U}}_{\epsilon}(n) 1_{m} .
\end{aligned}
$$

Indeed $\mathbf{S}_{\zeta}(n, m)$ depends only on $\zeta$ and not on the choice of $\epsilon$. If $\zeta$ is a primitive $d$-th root of 1 , we choose $\epsilon$ to be a primitive $2 d$-th root of 1 . Then the quantum Frobenius homomorphism $\mathrm{Fr}: \dot{\mathbf{U}}_{\epsilon}(n) \rightarrow \dot{\mathbf{U}}_{1}(n)$ factors through a $\mathbb{C}$-algebra homomorphism

$$
\operatorname{Fr}: \mathbf{S}_{\zeta}(n, d m) \rightarrow \mathbf{S}_{1}(n, m) \text {. }
$$

Note that we have used the identification $\dot{\mathbf{U}}_{(-1)^{d}}(n)=\dot{\mathbf{U}}_{1}(n)$ in Remark B.2.

B.3. The module category of $S_{\zeta}(n, m)$. Recall the set $\mathbb{Z}_{+}^{n}$ from Section 3.8. For $\lambda \in \mathbb{Z}_{+}^{n}$, let $\Delta_{\lambda}^{U}$ and $L_{\lambda}^{U}$ denote the Weyl module and the simple module with highest weight $\lambda$ in $\operatorname{Rep}\left(\dot{\mathbf{U}}_{\epsilon}(n)\right)$. See [31, [1] for the details. Set

$$
\Lambda(n, m)_{+}=\Lambda(n, m) \cap \mathbb{Z}_{+}^{n} .
$$

The category $\operatorname{Rep}\left(\mathbf{S}_{\zeta}(n, m)\right)$ is equivalent to the full subcategory of $\operatorname{Rep}\left(\dot{\mathbf{U}}_{\epsilon}(n)\right)$ consisting of the modules such that all constituents have a highest weight in the set $\Lambda(n, m)_{+}$. Parshall and Wang 31] were the first to show that the $\zeta$-Schur algebra is quasi-hereditary. It is quasi-hereditary with respect to the dominance order, the standard objects being the modules $\Delta_{\lambda}^{S}$ with $\lambda \in \Lambda(n, m)_{+}$. Here, for $\lambda \in \Lambda(n, m)_{+}$, we write

regarded as objects in $\operatorname{Rep}\left(\mathbf{S}_{\zeta}(n, m)\right)$.

$$
\Delta_{\lambda}^{S}=\Delta_{\lambda}^{U}, \quad L_{\lambda}^{S}=L_{\lambda}^{U},
$$

B.4. The Schur functor. Assume that $n \geqslant m$. There is a $\mathbb{C}$-algebra isomorphism [10, sec. 11]

$$
\mathbf{H}_{\zeta}(m)=f \mathbf{S}_{\zeta}(n, m) f, \quad f=1_{\left(1^{m} 0^{n-m}\right)} .
$$

Thus the vector space $\mathbf{T}_{\zeta}(n, m)=\mathbf{S}_{\zeta}(n, m) f$ is a $\left(\mathbf{S}_{\zeta}(n, m), \mathbf{H}_{\zeta}(m)\right)$-bimodule, and $\mathbf{V}_{\zeta}(n, m)=f \mathbf{S}_{\zeta}(n, m)$ is a $\left(\mathbf{H}_{\zeta}(m), \mathbf{S}_{\zeta}(n, m)\right)$-bimodule. Consider the triple of adjoint functors $\left(\Phi_{!}, \Phi^{*}, \Phi_{*}\right)$ :

$$
\begin{array}{ll}
\Phi^{*}: \operatorname{Rep}\left(\mathbf{S}_{\zeta}(n, m)\right) \rightarrow \operatorname{Rep}\left(\mathbf{H}_{\zeta}(m)\right), & M \mapsto f M, \\
\Phi_{*}: \operatorname{Rep}\left(\mathbf{H}_{\zeta}(m)\right) \rightarrow \operatorname{Rep}\left(\mathbf{S}_{\zeta}(n, m)\right), & N \mapsto \operatorname{Hom}_{\mathbf{H}_{\zeta}(m)}\left(\mathbf{V}_{\zeta}(n, m), N\right), \\
\Phi_{!}: \operatorname{Rep}\left(\mathbf{H}_{\zeta}(m)\right) \rightarrow \operatorname{Rep}\left(\mathbf{S}_{\zeta}(n, m)\right), & M \mapsto \mathbf{T}_{\zeta}(n, m) \otimes_{\mathbf{H}_{\zeta}(m)} M .
\end{array}
$$


We call $\Phi^{*}$ the Schur functor. It is a quotient functor; i.e., it is exact and the counit $\Phi^{*} \Phi_{*} \rightarrow 1$ is invertible. The double centralizer property holds; i.e., we have

$$
\mathbf{S}_{\zeta}(n, m)=\operatorname{End}_{\mathbf{H}_{\zeta}(m)}\left(\mathbf{V}_{\zeta}(n, m)\right) .
$$

Equivalently, the functor $\Phi^{*}$ is fully faithful on projectives, or, equivalently again, the unit $P \rightarrow \Phi_{*} \Phi^{*}(P)$ is invertible whenever $P$ is projective. See [32, prop. 4.33] for the details. Since $\Phi^{*}$ is a quotient functor, the functor $\Phi_{!}$takes projectives to projectives and the unit $1 \rightarrow \Phi^{*} \Phi_{\text {! }}$ is an isomorphism of functors. For $m=m^{\prime}+m^{\prime \prime}$ the comultiplication (B.4) yields a functor

$$
\dot{\otimes}: \operatorname{Rep}\left(\mathbf{S}_{\zeta}\left(n, m^{\prime}\right)\right) \otimes \operatorname{Rep}\left(\mathbf{S}_{\zeta}\left(n, m^{\prime \prime}\right)\right) \rightarrow \operatorname{Rep}\left(\mathbf{S}_{\zeta}(n, m)\right) .
$$

We'll abbreviate ${ }^{\mathbf{H}} \operatorname{Ind}_{m^{\prime}, m^{\prime \prime}}=\operatorname{Ind}_{\mathbf{H}_{\zeta}\left(m^{\prime}\right) \otimes \mathbf{H}_{\zeta}\left(m^{\prime \prime}\right)}^{\mathbf{H}_{\zeta}}$.

Proposition B.3. (a) We have a $\left(\mathbf{S}_{\zeta}(n, m), \mathbf{H}_{\zeta}\left(m^{\prime}\right) \otimes \mathbf{H}_{\zeta}\left(m^{\prime \prime}\right)\right)$-bimodule isomorphism can : $\mathbf{T}_{\zeta}\left(n, m^{\prime}\right) \dot{\otimes} \mathbf{T}_{\zeta}\left(n, m^{\prime \prime}\right) \rightarrow \mathbf{T}_{\zeta}(n, m)$. For $M^{\prime} \in \operatorname{Rep}\left(\mathbf{H}_{\zeta}\left(m^{\prime}\right)\right), M^{\prime \prime} \in$ $\operatorname{Rep}\left(\mathbf{H}_{\zeta}\left(m^{\prime \prime}\right)\right)$ the map can yield an isomorphism

$$
\text { can : } \Phi_{!}\left({ }^{\mathbf{H}} \operatorname{Ind}_{m^{\prime}, m^{\prime \prime}}\left(M^{\prime} \otimes M^{\prime \prime}\right)\right) \rightarrow \Phi_{!}\left(M^{\prime}\right) \dot{\otimes} \Phi_{!}\left(M^{\prime \prime}\right) .
$$

(b) We have an isomorphism of $\left(\mathbf{H}_{\zeta}\left(m^{\prime}\right) \otimes \mathbf{H}_{\zeta}\left(m^{\prime \prime}\right), \mathbf{S}_{\zeta}(n, m)\right)$-bimodules can : $\mathbf{V}_{\zeta}\left(n, m^{\prime}\right) \dot{\otimes} \mathbf{V}_{\zeta}\left(n, m^{\prime \prime}\right) \rightarrow \mathbf{V}_{\zeta}(n, m)$. For $M^{\prime} \in \operatorname{Rep}\left(\mathbf{H}_{\zeta}\left(m^{\prime}\right)\right), M^{\prime \prime} \in \operatorname{Rep}\left(\mathbf{H}_{\zeta}\left(m^{\prime \prime}\right)\right)$ the map can yields an isomorphism

$$
\text { can : } \Phi_{*}\left({ }^{\mathbf{H}} \operatorname{Ind}_{m^{\prime}, m^{\prime \prime}}\left(M^{\prime} \otimes M^{\prime \prime}\right)\right) \rightarrow \Phi_{*}\left(M^{\prime}\right) \dot{\otimes} \Phi_{*}\left(M^{\prime \prime}\right) .
$$

Proof. By definition $\mathbf{T}_{\zeta}(n, m)$ is the v-tensor space in [8, def. 2.6]. According to [7, sec. 3.3, 4.4] it is identified with the $m$-th tensor power of the natural representation of the (modified) quantized enveloping algebra of $\mathfrak{g l}_{n}$, in such a way that the $\mathbf{H}_{\zeta}(m)$ action comes from the R-matrix; see also [17. This proves part $(a)$. Part $(b)$ follows also by taking the dual spaces.

Corollary B.4. We have an isomorphism

$$
\text { can : }{ }^{\mathbf{H}} \operatorname{Ind}_{m^{\prime}, m^{\prime \prime}}\left(\Phi^{*} M^{\prime} \otimes \Phi^{*} M^{\prime \prime}\right) \rightarrow \Phi^{*}\left(M^{\prime} \dot{\otimes} M^{\prime \prime}\right)
$$

for $M^{\prime} \in \operatorname{Rep}\left(\mathbf{S}_{\zeta}\left(n, m^{\prime}\right)\right)$ and $M^{\prime \prime} \in \operatorname{Rep}\left(\mathbf{S}_{\zeta}\left(n, m^{\prime \prime}\right)\right)$.

Proof. For $M^{\prime} \in \operatorname{Rep}\left(\mathbf{S}_{\zeta}\left(n, m^{\prime}\right)\right)$ and $M^{\prime \prime} \in \operatorname{Rep}\left(\mathbf{S}_{\zeta}\left(n, m^{\prime \prime}\right)\right)$, Proposition B.3 yields an isomorphism

$$
\Phi_{*}{ }^{\mathbf{H}} \operatorname{Ind}_{m^{\prime}, m^{\prime \prime}}\left(\Phi^{*} M^{\prime} \otimes \Phi^{*} M^{\prime \prime}\right)=\Phi_{*} \Phi^{*} M^{\prime} \dot{\otimes} \Phi_{*} \Phi^{*} M^{\prime \prime} .
$$

Composing it with $\Phi^{*}$ we get an isomorphism

$$
{ }^{\mathbf{H}} \operatorname{Ind}_{m^{\prime}, m^{\prime \prime}}\left(\Phi^{*} M^{\prime} \otimes \Phi^{*} M^{\prime \prime}\right)=\Phi^{*}\left(\Phi_{*} \Phi^{*} M^{\prime} \dot{\otimes} \Phi_{*} \Phi^{*} M^{\prime \prime}\right) .
$$

Composing it with the unit $1 \rightarrow \Phi_{*} \Phi^{*}$ we get a functorial map

$$
\Phi^{*}\left(M^{\prime} \dot{\otimes} M^{\prime \prime}\right) \rightarrow{ }^{\mathbf{H}} \operatorname{Ind}_{m^{\prime}, m^{\prime \prime}}\left(\Phi^{*} M^{\prime} \otimes \Phi^{*} M^{\prime \prime}\right),
$$

which is invertible whenever $M^{\prime}, M^{\prime \prime}$ are projectives, because the unit is invertible on projective modules. Thus it is always invertible, because $\Phi^{*}$ and ${ }^{\mathbf{H}} \operatorname{Ind}_{m^{\prime}, m^{\prime \prime}}$ are exact and because there are enough projectives in $\operatorname{Rep}\left(\mathbf{S}_{\zeta}(n, m)\right)$. 
B.5. The braiding and the Schur functor. For $M^{\prime} \in \operatorname{Rep}\left(\mathbf{H}_{\zeta}\left(m^{\prime}\right)\right)$ and $M^{\prime \prime} \in$ $\operatorname{Rep}\left(\mathbf{H}_{\zeta}\left(m^{\prime \prime}\right)\right)$ the R-matrix yields an isomorphism of $\mathbf{S}_{\zeta}(n, m)$-modules

$$
\mathcal{R}_{\Phi_{*} M^{\prime}, \Phi_{*} M^{\prime \prime}}: \Phi_{*} M^{\prime} \dot{\otimes} \Phi_{*} M^{\prime \prime} \rightarrow \Phi_{*} M^{\prime \prime} \dot{\otimes} \Phi_{*} M^{\prime} .
$$

Let $\tau \in \mathfrak{S}_{m}$ be the unique element such that

- $\tau$ is minimal in the coset $\left(\mathfrak{S}_{m^{\prime}} \times \mathfrak{S}_{m^{\prime \prime}}\right) \tau\left(\mathfrak{S}_{m^{\prime \prime}} \times \mathfrak{S}_{m^{\prime}}\right)$,

- we have $\tau^{-1}\left(\mathfrak{S}_{m^{\prime}} \times \mathfrak{S}_{m^{\prime \prime}}\right) \tau=\mathfrak{S}_{m^{\prime \prime}} \times \mathfrak{S}_{m^{\prime}}$.

We have the following formula in $\mathbf{H}_{\zeta}(m)$ :

$$
T_{\tau}\left(h^{\prime \prime} \otimes h^{\prime}\right)=\left(h^{\prime} \otimes h^{\prime \prime}\right) T_{\tau}, \quad h^{\prime} \in \mathbf{H}_{\zeta}\left(m^{\prime}\right), h^{\prime \prime} \in \mathbf{H}_{\zeta}\left(m^{\prime \prime}\right) .
$$

Thus there is a unique functorial $\mathbf{H}_{\zeta}(m)$-module isomorphism

$$
\mathcal{S}_{M^{\prime}, M^{\prime \prime}}:{ }^{\mathbf{H}} \operatorname{Ind}_{m^{\prime}, m^{\prime \prime}}\left(M^{\prime} \otimes M^{\prime \prime}\right) \rightarrow{ }^{\mathbf{H}} \operatorname{Ind}_{m^{\prime \prime}, m^{\prime}}\left(M^{\prime \prime} \otimes M^{\prime}\right)
$$

given by

$$
\mathcal{S}_{M^{\prime}, M^{\prime \prime}}\left(h \otimes\left(v^{\prime} \otimes v^{\prime \prime}\right)\right)=h T_{\tau} \otimes\left(v^{\prime \prime} \otimes v^{\prime}\right), \quad h \in \mathbf{H}_{\zeta}(m), v^{\prime} \in M^{\prime}, v^{\prime \prime} \in M^{\prime \prime} .
$$

Proposition B.5. For $M^{\prime} \in \operatorname{Rep}\left(\mathbf{H}_{\zeta}\left(m^{\prime}\right)\right), M^{\prime \prime} \in \operatorname{Rep}\left(\mathbf{H}_{\zeta}\left(m^{\prime \prime}\right)\right)$ the following square is commutative:

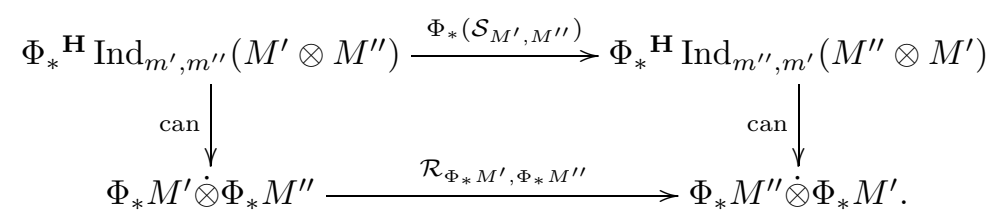

Proof. We abbreviate $\mathbf{H}=\mathbf{H}_{\zeta}(m), \mathbf{H}^{\prime}=\mathbf{H}_{\zeta}\left(m^{\prime}\right), \mathbf{H}^{\prime \prime}=\mathbf{H}_{\zeta}\left(m^{\prime \prime}\right), \mathbf{V}=\mathbf{V}_{\zeta}(n, m)$, $\mathbf{V}^{\prime}=\mathbf{V}_{\zeta}\left(n, m^{\prime}\right)$ and $\mathbf{V}^{\prime \prime}=\mathbf{V}_{\zeta}\left(n, m^{\prime \prime}\right)$. First, we have a commutative square

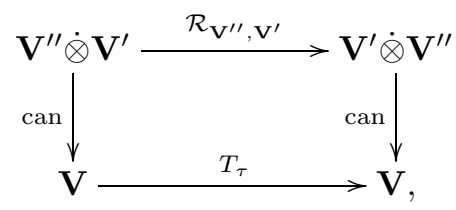

where the lower map is the left multiplication with $T_{\tau}$. See [17] and the discussion in the proof of Proposition B.3. In particular, we have

$$
\begin{gathered}
\mathcal{R}_{\mathbf{V}^{\prime \prime}, \mathbf{V}^{\prime}}\left(h^{\prime \prime} v^{\prime \prime} \otimes h^{\prime} v^{\prime}\right)=\left(h^{\prime \prime} \otimes h^{\prime}\right) \mathcal{R}_{\mathbf{V}^{\prime \prime}, \mathbf{V}^{\prime}}\left(v^{\prime} \otimes v^{\prime \prime}\right), \\
v^{\prime} \in \mathbf{V}^{\prime}, v^{\prime \prime} \in \mathbf{V}^{\prime \prime}, h^{\prime} \in \mathbf{H}^{\prime}, h^{\prime \prime} \in \mathbf{H}^{\prime \prime} .
\end{gathered}
$$

Therefore, the composition with $\mathcal{R}_{\mathbf{V}^{\prime \prime}, \mathbf{V}^{\prime}}$ yields a linear map

$$
\begin{aligned}
& \operatorname{Hom}_{\mathbf{H}^{\prime} \otimes \mathbf{H}^{\prime \prime}}\left(\mathbf{V}, M^{\prime} \otimes M^{\prime \prime}\right)=\Phi_{*}{ }^{\mathbf{H}} \operatorname{Ind}_{m^{\prime}, m^{\prime \prime}}\left(M^{\prime} \otimes M^{\prime \prime}\right) \\
\rightarrow & \operatorname{Hom}_{\mathbf{H}^{\prime \prime} \otimes \mathbf{H}^{\prime}}\left(\mathbf{V}, M^{\prime \prime} \otimes M^{\prime}\right)=\Phi_{*}{ }^{\mathbf{H}} \operatorname{Ind}_{m^{\prime \prime}, m^{\prime}}\left(M^{\prime \prime} \otimes M^{\prime}\right) .
\end{aligned}
$$

The commutativity of the square (B.8) implies that this map is equal to $\Phi_{*}\left(\mathcal{S}_{M^{\prime}, M^{\prime \prime}}\right)$. It is easy to see that this map also coincides with $\mathcal{R}_{\Phi_{*} M^{\prime}, \Phi_{*} M^{\prime \prime}}$. 
Corollary B.6. For $M^{\prime} \in \operatorname{Rep}\left(\mathbf{S}_{\zeta}\left(n, m^{\prime}\right)\right), M^{\prime \prime} \in \operatorname{Rep}\left(\mathbf{S}_{\zeta}\left(n, m^{\prime \prime}\right)\right)$ the following square is commutative:

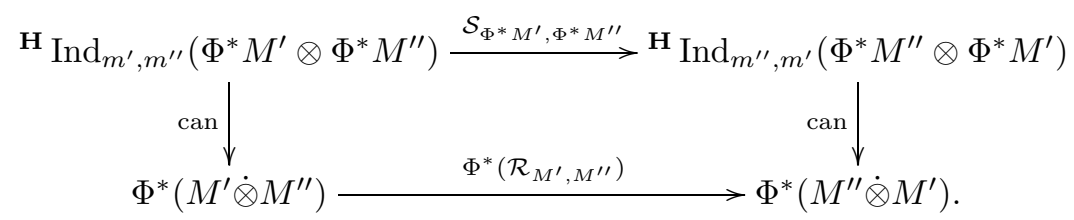

Proof. Use the same argument as in the proof of Corollary B.4.

Let $r \geqslant 1$ and $i=1,2, \ldots, r-1$. For $M \in \operatorname{Rep}\left(\mathbf{H}_{\zeta}(m)\right)$ we consider the automorphism of the $\mathbf{H}_{\zeta}(m r)$-module ${ }^{\mathbf{H}} \operatorname{Ind}_{\left(m^{r}\right)}\left(M^{\otimes r}\right)$ given by

$$
\begin{gathered}
\mathcal{S}_{M, i}={ }^{\mathbf{H}} \operatorname{Ind}_{\mathbf{H}}^{\mathbf{H}_{\zeta}(m r)}\left(\mathbf{1}^{\otimes i-1} \otimes \mathcal{S}_{M, M} \otimes \mathbf{1}^{\otimes r-i-1}\right), \\
\mathbf{H}=\mathbf{H}_{\zeta}(m)^{\otimes i-1} \otimes \mathbf{H}_{\zeta}(2 m) \otimes \mathbf{H}_{\zeta}(m)^{\otimes r-i-1} .
\end{gathered}
$$

For $M \in \operatorname{Rep}\left(\mathbf{S}_{\zeta}(n, m)\right)$ we consider the automorphism of the $\mathbf{S}_{\zeta}(n, m r)$-module $M^{\dot{\otimes} r}$ given by

$$
\mathcal{R}_{M, i}=\mathbf{1}^{\dot{\otimes} i-1} \dot{\otimes} \mathcal{R}_{M, M} \dot{\otimes} \mathbf{1}^{\dot{\otimes} r-i-1} .
$$

Corollary B.7. For $M \in \operatorname{Rep}\left(\mathbf{S}_{\zeta}(n, m)\right), r \geqslant 1$ and $i=1,2, \ldots, r-1$ we have $a$ commutative square with invertible vertical maps

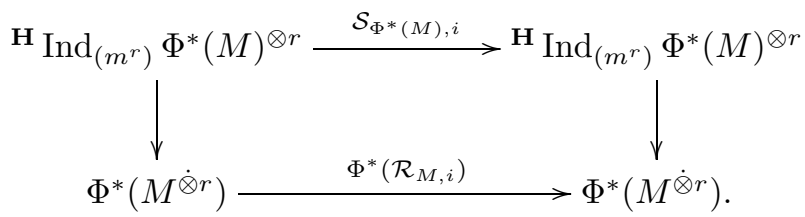

B.6. The braiding and the quantum Frobenius homomorphism. Recall that if $\zeta$ is a primitive $d$-th root of 1 , then the quantum Frobenius homomorphism (B.5) yields a functor

$$
\operatorname{Fr}^{*}: \operatorname{Rep}\left(\mathbf{S}_{1}(n, m)\right)=\operatorname{Rep}\left(\mathbf{S}_{(-1)^{d}}(n, m)\right) \rightarrow \operatorname{Rep}\left(\mathbf{S}_{\zeta}(n, d m)\right) .
$$

Here we have identified $\mathbf{S}_{(-1)^{d}}(n, m)$ and $\mathbf{S}_{1}(n, m)$ as in Remark B.2. Let $m^{\prime}, m^{\prime \prime}>$ 0 with $m=m^{\prime}+m^{\prime \prime}$. By Proposition B.1 for $M \in \operatorname{Rep}\left(\mathbf{S}_{(-1)^{d}}(n, m)\right), M^{\prime} \in$ $\operatorname{Rep}\left(\mathbf{S}_{(-1)^{d}}\left(n, m^{\prime}\right)\right)$ the braiding operator

$$
\mathcal{R}_{M, M^{\prime}}: M \dot{\otimes} M^{\prime} \rightarrow M^{\prime} \dot{\otimes} M
$$

is the composition of the permutation $\mathbf{s}_{M, M^{\prime}}$ and of the operator

$$
R_{M, M^{\prime}}=\prod_{\lambda, \lambda^{\prime}}(-1)^{d\left(\lambda, \lambda^{\prime}\right)}\left(1_{\lambda} \dot{\otimes} 1_{\lambda^{\prime}}\right) .
$$

Proposition B.8. For $r \geqslant 1, i, j=1,2, \ldots, r-1$, and $M \in \operatorname{Rep}\left(\mathbf{S}_{(-1)^{d}}(n, m)\right)$ the following relations hold in $\operatorname{End}_{\mathbf{S}_{(-1) d}(n, m r)}\left(M^{\dot{\otimes} r}\right)$ :

- $\mathcal{R}_{M, i}^{2}=1$,

- $\mathcal{R}_{M, i} \mathcal{R}_{M, j}=\mathcal{R}_{M, j} \mathcal{R}_{M, i}$ if $j \neq i-1, i+1$,

- $\mathcal{R}_{M, i} \mathcal{R}_{M, i+1} \mathcal{R}_{M, i}=\mathcal{R}_{M, i+1} \mathcal{R}_{M, i} \mathcal{R}_{M, i+1}$ if $i \neq r-1$. 
Proof. The first relation is obvious by the definition of the braiding operator; see above. The other relations are consequences of the general properties of a braiding.

Further, the functor $\mathrm{Fr}^{*}$ is a braided tensor functor; i.e., we have the following.

Proposition B.9. For $M \in \operatorname{Rep}\left(\mathbf{S}_{(-1)^{d}}\left(n, m^{\prime}\right)\right), M^{\prime} \in \operatorname{Rep}\left(\mathbf{S}_{(-1)^{d}}\left(n, m^{\prime \prime}\right)\right)$ we have a functorial isomorphism $\operatorname{Fr}^{*}\left(M \dot{\otimes} M^{\prime}\right)=\operatorname{Fr}^{*}(M) \dot{\otimes} \operatorname{Fr}^{*}\left(M^{\prime}\right)$ in $\operatorname{Rep}\left(\mathbf{S}_{\zeta}(n, d m)\right)$ such that $\operatorname{Fr}^{*}\left(\mathcal{R}_{M, M^{\prime}}\right)=\mathcal{R}_{\operatorname{Fr}^{*}(M), \operatorname{Fr}^{*}\left(M^{\prime}\right)}$.

Proof. This is obvious by Proposition B.1.

B.7. The algebra $S_{\zeta}(m)$. We'll abbreviate $\mathbf{S}_{\zeta}(m)=\mathbf{S}_{\zeta}(m, m)$. If $n \geqslant m$ the algebra $\mathbf{S}_{\zeta}(n, m)$ is Morita equivalent to $\mathbf{S}_{\zeta}(m)$; see, e.g., [8, lem. 1.3]. Thus $\dot{\otimes}$ can be viewed as a functor (choosing $n \geqslant m=m^{\prime}+m^{\prime \prime}$ )

$$
\dot{\otimes}: \operatorname{Rep}\left(\mathbf{S}_{\zeta}\left(m^{\prime}\right)\right) \otimes \operatorname{Rep}\left(\mathbf{S}_{\zeta}\left(m^{\prime \prime}\right)\right) \rightarrow \operatorname{Rep}\left(\mathbf{S}_{\zeta}(m)\right)
$$

If $\zeta$ is a primitive $d$-th root of 1 , then the quantum Frobenius homomorphism can be viewed as a functor (choosing $n \geqslant d m$ )

$$
\operatorname{Fr}^{*}: \operatorname{Rep}\left(\mathbf{S}_{1}(m)\right)=\operatorname{Rep}\left(\mathbf{S}_{(-1)^{d}}(m)\right) \rightarrow \operatorname{Rep}\left(\mathbf{S}_{\zeta}(d m)\right) .
$$

\section{Appendix C. Remarks on the Fock SPACE}

We record here some remarks concerning the Fock space. They have not been used in the paper. First, there is a tautological $\mathbb{C}$-linear isomorphism $\mathbb{C}^{m} \otimes \mathbb{C}^{\ell}=$ $\mathbb{C}^{m \ell}$. It yields $\mathbb{C}$-linear isomorphisms $V_{m, \ell} \rightarrow V_{m \ell}$ and $\mathcal{F}_{m, \ell} \rightarrow \mathcal{F}_{m \ell}$. Recall that $\mathcal{F}_{m \ell}$ is equipped with a level 1 action of $\widehat{\mathfrak{s l}}_{m \ell}$, and that $\mathcal{F}_{m, \ell}$ is equipped with a level $(\ell, m)$ action of $\widehat{\mathfrak{s l}}_{m} \times \widehat{\mathfrak{s l}}_{\ell}$. Now, there is a well-known Lie algebra inclusion

$$
\left(\widehat{\mathfrak{s l}}_{m} \times \widehat{\mathfrak{s l}}_{\ell}\right) /(m(\mathbf{1}, 0)-\ell(0, \mathbf{1})) \subset \widehat{\mathfrak{s l}}_{m \ell}, \quad(\mathbf{1}, 0) \mapsto \ell \mathbf{1}, \quad(0, \mathbf{1}) \mapsto m \mathbf{1} .
$$

This inclusion intertwines the $\widehat{\mathfrak{s l}}_{m} \times \widehat{\mathfrak{s l}}_{\ell}$ action on $\mathcal{F}_{m, \ell}$ and the $\widehat{\mathfrak{s l}}_{m \ell}$ action on $\mathcal{F}_{m, \ell}=\mathcal{F}_{m \ell}$. Further, we want to compare the $\widehat{\mathfrak{s l}}_{m \ell}$ action on $\mathcal{F}_{m, \ell}$ with the level one $\widehat{\mathfrak{s l}}_{\ell}$ action on $\mathcal{F}_{m, \ell}$ given in the beginning of this section. The $\mathbb{C}$-linear isomorphisms (6.16) and (6.17) yield a $\mathbb{C}$-linear isomorphism

$$
\mathcal{F}_{\ell} \rightarrow \mathcal{F}_{m, \ell}=\mathcal{F}_{m \ell}
$$

The right-hand side is equipped with a level 1 action of $\widehat{\mathfrak{s l}}_{m \ell}$, and the left-hand side with a level 1 action of $\widehat{\mathfrak{s l}}_{\ell}$. Consider the following elements in $\mathfrak{s l}_{m} \otimes \mathbb{C}\left[\varpi, \varpi^{-1}\right]$ :

$$
\begin{gathered}
x(i+k m)=\sum_{j=1}^{m-i} e_{j, i+j} \otimes \varpi^{k}+\sum_{j=m-i+1}^{m} e_{j, i+j-m} \otimes \varpi^{k+1}, \\
1 \leqslant i \leqslant m, \quad k \in \mathbb{Z} .
\end{gathered}
$$

For $x \in \mathfrak{s l}_{m} \otimes \mathbb{C}\left[\varpi, \varpi^{-1}\right]$ and $p, q=1,2, \ldots, \ell$ we define the element $x^{(p, q)} \in$ $\mathfrak{s l}_{m \ell} \otimes \mathbb{C}\left[\varpi, \varpi^{-1}\right]$ by

$$
x^{(p, q)}=\sum_{i, j=1}^{m} e_{i+(p-1) m, j+(q-1) m} \otimes a_{i, j} \quad \text { for } \quad x=\sum_{i, j=1}^{m} e_{i, j} \otimes a_{i, j} .
$$


The following claim is proved by a direct computation, which is left to the reader.

Proposition C.1. (a) There is a Lie algebra inclusion $\widehat{\mathfrak{s l}}_{\ell} \subset \widehat{\mathfrak{s l}}_{m \ell}$ given by

$$
\mathbf{1} \mapsto \mathbf{1}, \quad e_{p, q} \otimes \varpi^{r} \mapsto x(r)^{(p, q)}, \quad p, q=1,2, \ldots, \ell, \quad r \in \mathbb{Z} .
$$

(b) The map (C.1) intertwines the $\widehat{\mathfrak{s l}}_{\ell}$ action on $\mathcal{F}_{\ell}$ and the $\widehat{\mathfrak{s l}}_{m \ell}$ action on $\mathcal{F}_{m \ell}$.

\section{INDEX OF NOTATION}

1.3: $Z(\mathcal{A}), \operatorname{Irr}(\mathcal{A}), \operatorname{Rep}(\mathbf{A}), \operatorname{Irr}(\mathbf{A}),[\mathcal{A}], D^{b}(\mathcal{A}), D^{b}(\mathbf{A}),\langle m\rangle, \mathcal{A} \otimes \mathcal{B}, \mathbf{1}_{F}$.

2.1: $W, \mathfrak{h}, c, H(W), R_{x}, R_{y}, \mathcal{O}(W), \Delta_{\chi}, \mathcal{O}(W, \mathfrak{h}), H(W, \mathfrak{h})$.

2.2: $X_{W^{\prime}}^{\circ}, X_{W^{\prime}}, X_{W^{\prime}, \mathfrak{h}}^{\circ}, X_{W^{\prime}, \mathfrak{h}}$.

2.3: $W_{b}, \pi_{b}, \operatorname{Ind}_{b}, \operatorname{Res}_{b},{ }^{\mathcal{O}} \operatorname{Ind}_{W_{b}}^{W},{ }^{\mathcal{O}} \operatorname{Res}_{W_{b}}^{W}, \operatorname{Supp}(M)$, spe, ${ }^{x} M$.

3.1: $|\lambda|, \Lambda(\ell, n), \mathcal{P}_{n}, \lambda^{\prime}, l(\lambda), z_{\lambda}, m \lambda, \mathbb{Z}_{\ell}, \mathcal{P}^{\ell}, \mathcal{P}_{n}^{\ell}, \Gamma, \mathcal{P}^{\Gamma}, \mathcal{P}_{n}^{\Gamma}$.

3.2: $\mathfrak{S}_{n}, \Gamma_{n}, \gamma_{i}, s_{i j}, s_{i}, s_{i j}^{\gamma}, \chi_{p}, \mathbb{C}_{0}^{n}, \Gamma_{\nu}, \Gamma_{n, \nu}, \Gamma_{n, m}$.

3.3: $x_{i}, y_{i}, H\left(\Gamma_{n}\right), h, h_{p}$.

3.4: $\Lambda, \omega_{p}, \widehat{\mathfrak{s l}}_{\ell}, \mathbf{1}, e_{p}, f_{p}, e_{p, q}, \widetilde{\mathfrak{s l}}_{\ell}, D, U\left(\widehat{\mathfrak{s l}}_{\ell}\right), U^{-}\left(\widehat{\mathfrak{s l}}_{\ell}\right), U^{-}\left(\widehat{\mathfrak{s l}}_{\ell}\right)_{r}$.

3.5: $\bar{L}_{\lambda}$.

3.6: $\Delta_{\lambda}, L_{\lambda}, P_{\lambda},{ }^{\mathcal{O}} \operatorname{Ind}_{n},{ }^{\mathcal{O}} \operatorname{Res}_{n},{ }^{\mathcal{O}} \operatorname{Ind}_{n,\left(m^{r}\right)},{ }^{\mathcal{O}} \operatorname{Res}_{n,\left(m^{r}\right)},{ }^{\mathcal{O}} \operatorname{Ind}_{n, m r},{ }^{\mathcal{O}} \operatorname{Res}_{n, m r}$, ${ }^{\mathcal{O}} \operatorname{Ind}_{\left(m^{r}\right)},{ }^{\mathcal{O}} \operatorname{Res}\left(m^{r}\right)$.

3.7: $\mathbf{H}\left(\Gamma_{n}\right), \zeta, v_{p}, \mathrm{KZ}, S$.

3.8: $\mathbf{H}_{\zeta}(m), \mathbf{S}_{\zeta}(m), \Phi^{*}, \Lambda(m)_{+}, \mathbb{Z}_{+}^{m}, \Delta_{\lambda}^{S}, \dot{\otimes}, R, \mathcal{O}(\mathfrak{S}), \operatorname{Rep}\left(\mathbf{S}_{\zeta}\right)$.

3.9: $D_{n}(z), b_{n}, e_{q}, f_{q}, E, F, \tilde{e}_{q}, \tilde{f}_{q}, s=\left(s_{p}\right), \mathcal{F}_{m, \ell}^{(s)}$.

3.10: $X_{l, \nu}^{\circ}, X_{l, \nu}, X_{l, j}^{\circ}, X_{l, j}, X_{l, j, \mathbb{C}^{n}}, \operatorname{Irr}\left(\mathcal{O}\left(\Gamma_{n}\right)\right)_{i, j}, F_{i, j}\left(\Gamma_{n}\right), \operatorname{gr}\left(\Gamma_{n}\right), \operatorname{gr}_{i, j}\left(\Gamma_{n}\right)$, $F_{i, \bullet}\left(\Gamma_{n}\right), F_{\bullet}, j\left(\Gamma_{n}\right), \operatorname{gr}_{i, \bullet}\left(\Gamma_{n}\right), \operatorname{gr}_{\bullet}\left(\Gamma_{n}\right), F_{i, j}\left(\Gamma_{n}\right)^{\circ}$.

3.11: $E, F$.

4.1: $R(\mathfrak{S}), R(\Gamma), \boldsymbol{\Lambda}, \mathrm{ch}, S_{\lambda}, P_{r}, P_{\lambda}$.

4.2: $\mathfrak{H}, \mathbf{1}, b_{r}, b_{r}^{\prime}, U(\mathfrak{H}), U^{-}(\mathfrak{H}), U^{-}(\mathfrak{H})_{r}, b_{\lambda}, b_{\lambda}^{\prime}, b_{f}, b_{f}^{\prime}, V_{\ell}^{\mathfrak{H}}, \partial, V_{\ell}^{\mathfrak{H}}[j]$.

4.3: $\widehat{\mathfrak{g l}}_{m}, \widetilde{\mathfrak{g l}}_{m}, P^{\widehat{\mathfrak{s l}}_{m}}, P^{\widetilde{\mathfrak{s l}}_{m}}, P^{\widehat{\mathfrak{g l}}_{m}}, P^{\widetilde{\mathfrak{g l}}_{m}}, P_{+}^{\widehat{\mathfrak{g l}}_{m}}, P_{+}^{\widetilde{\mathfrak{g} l_{m}}}, P_{+}^{\widehat{\mathfrak{s l}}_{m}}, P_{+}^{\widetilde{\mathfrak{s l}}_{m}}, V_{\lambda}^{\widetilde{\mathfrak{s l}}_{m}}, V_{\lambda}^{\widetilde{\mathfrak{g l}}_{m}}$, $Q^{\mathfrak{s l}_{m}}, P^{\mathfrak{s l}_{m}}, V[\mu], T_{m}, \mathfrak{t}_{m}, \widehat{\mathfrak{S}}_{m}, \xi_{\beta}, \beta(\varpi)$.

4.4: $\Lambda_{\Gamma}, f^{\gamma}, P_{\mu}^{\gamma}, P_{\lambda}, P_{r, p}, S_{\mu, p}, S_{\lambda}, \operatorname{ch}, \tau, z_{\lambda}, \bar{\lambda}$.

4.5: $\epsilon_{i}, \mathcal{F}_{m}, V_{m}, \mathcal{F}_{m}^{(d)}, u_{i},|\lambda, d\rangle$.

4.6: $\dot{\epsilon}_{i}, \mathcal{F}_{m, \ell}, V_{m, \ell}, u_{i}, \hat{\gamma}(s, m), \mathcal{F}_{m, \ell}^{(s)},|\lambda, s\rangle, n_{q}(\lambda), \Delta(s, m)$.

5.1: $\mathcal{O}(\Gamma), b_{n, r}, \operatorname{Hom}_{\mathcal{O}\left(\mathfrak{S}_{m r}\right)}\left(\bullet, L_{m \lambda}\right)^{\circledast}, A_{\lambda, !}, A_{\lambda}^{*}, A_{\lambda, *}$.

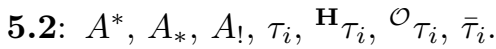

5.3: $R a_{\lambda, *}, R a_{\lambda, !}, R^{i} a_{\lambda, *}, R^{i} a_{\lambda, !}, a_{\lambda}^{*}, a_{\lambda, *}, a_{\lambda, !}$.

5.4: $\operatorname{PI}\left(\mathcal{O}\left(\Gamma_{n}\right)\right)$.

5.5: $B_{r}, B_{r, \ell},{ }^{\mathbf{H}_{\xi}} \xi_{i}{ }^{\mathcal{O}} \xi_{i}, \bar{\xi}_{i}, \bar{\tau}_{w}, \bar{a}_{\lambda}^{*}, \Psi$.

5.6: $\tilde{a}_{\lambda}(L)$.

6.2: $[\mathcal{O}(\Gamma)]_{\bullet, j},[\mathcal{O}(\Gamma)]_{i, \bullet},[\mathcal{O}(\Gamma)]_{i, j},\left[\mathcal{O}\left(\Gamma_{n}\right)\right]_{i, j}$.

6.3: $\alpha_{p, q}, \tilde{\mathfrak{a}}_{\Lambda}, \Delta_{+}^{\tilde{\mathfrak{a}}}, P_{+}^{\tilde{\mathfrak{a}}}, V_{\mu}^{\tilde{\mathfrak{a}}}, \partial_{m}, V_{\mu}^{\tilde{\mathfrak{a}}}[\lambda, j]$. 
6.4: $\tilde{\gamma}(\lambda, m), A(\ell, m), A(\ell, m)_{d}, \lambda^{\dagger}$.

6.5: $\lambda^{*}, \lambda^{c}, \tau\left(\lambda^{c}\right), c_{\lambda}(X)$.

6.6: $\mu^{\prime}, \widehat{\mathfrak{S}}_{\ell}^{e t}, \tilde{\omega}_{d}$.

A.1: $\hat{\mathbf{H}}_{\zeta}(n), X_{i}, T_{i}, \hat{\mathbf{H}}_{\zeta}(I), T_{w}, T_{i j}, D_{I}, D_{I, J}$.

A.2: $\mathbf{H}_{\zeta}(n, \ell), T_{0}, \Gamma_{I}, \mathbf{H}_{\zeta}(I, \ell), \mathbf{H}\left(\Gamma_{n}\right), \mathbf{H}\left(\mathfrak{S}_{m}\right), \mathbf{H}\left(\Gamma_{I}\right)$.

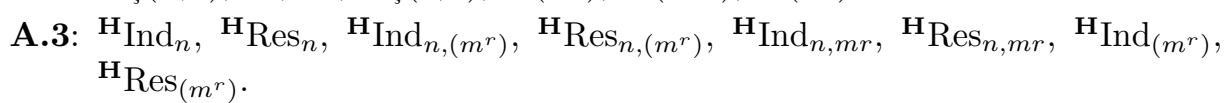

B.1: $\dot{\mathbf{U}}(n), 1_{\lambda},[m], \Delta, \mathcal{A}, \epsilon, \mathbf{s}_{V, V^{\prime}}, R_{V, V^{\prime}}, \mathcal{R}_{V, V^{\prime}}, \bar{\Pi}, \bar{\Theta}$, Fr.

B.2: $\mathbf{S}(n, m), \mathbf{S}_{\mathcal{A}}(n, m), \mathbf{S}_{\zeta}(n, m)$.

B.3: $\Delta_{\lambda}^{U}, L_{\lambda}^{U}, \Lambda(n, m)_{+}, \Delta_{\lambda}^{S}, L_{\lambda}^{S}$.

B.4: $f, \Phi^{*}, \Phi_{*}, \Phi_{!}, \dot{\otimes}$.

B.5: $\mathcal{S}_{M^{\prime}, M^{\prime \prime}}$.

B.6: $\mathrm{Fr}^{*}$.

B.7: $\mathbf{S}_{\zeta}(m)$.

\section{ACKNowledgments}

The authors are grateful to I. Losev for a careful reading of a preliminary draft of the paper.

\section{REFERENCES}

[1] Andersen, H.H., Polo, P., Wen, K., Representations of quantum algebras, Invent. Math., 104 (1991), 1-60. MR1094046 (92e:17011)

[2] Bezrukavnikov, R., Etingof, P., Parabolic induction and restriction functors for rational Cherednik algebras, Selecta Math. (N.S.) 14 (2009), 397-425. MR2511190 (2010e:20007)

[3] Berest, Y., Etingof, P., Ginzburg, V., Finite dimensional representations of rational Cherednik algebras, Int. Math. Res. Not. 19 (2003), 1053-1088. MR1961261 (2004h:16027)

[4] Carter, R., Lie algebras of finite and affine type, Cambridge University Press, Cambridge, 2005. MR2188930(2006i:17001)

[5] Cherednik, I., Double affine Hecke algebras, London Mathematical Society Lecture Note Series 319, Cambridge University Press, Cambridge, 2005. MR2133033 (2007e:32012)

[6] Deligne, P., Catégories tannakiennes, The Grothendieck Festschrift, Vol. II, 111-195, Progr. Math., 87, Birkhäuser Boston, Boston, MA, 1990. MR1106898 (92d:14002)

[7] Dipper, R., Donkin, S. Quantum $G L_{n}$, Proc. London Math. Soc. 63 (1991), 165-211. MR:1105721 (92g:16055)

[8] Dipper, R., James, G., q-Tensor Space and $q$-Weyl Modules, Trans. Amer. Math. Soc. 327 (1991), 251-282. MR.1012527 (91m:20061)

[9] Donkin, S., The q-Schur Algebra, London Mathematical Society Lecture Note Series, 253, Cambridge University Press, Cambridge, 1998. MR1707336 (2001h:20072)

[10] Doty, S., Giaquinto, A. Presenting Schur algebras, Int. Math. Res. Not. 36 (2002), 19071944. MR 1920169 (2004e:16037)

[11] Etingof, P., Symplectic reflection algebras and affine Lie algebras, arXiv:1011.4584.

[12] Frenkel, I, Jing, N., Wang, W., Vertex representations via finite groups and the McKay correspondence, Internat. Math. Res. Notices 4 (2000), 195-222. MR1747618 (2001c:17042)

[13] Ginzburg, V., On primitive ideals, Selecta Math. (N.S.) 9 (2003), 379-407. MR2006573 (2005f:16039)

[14] Ginzburg, V., Guay, N., Opdam, E., Rouquier, R., On the category O for rational Cherednik algebras, Invent. Math. 154 (2003), 617-651. MR2018786 (2005f:20010)

[15] Gordon, I., Quiver varieties, category $\mathcal{O}$ for rational Cherednik algebras and Hecke algebras, Int. Math. Res. Pap. 3 (2008). MR2457847 (2010c:16032) 
[16] Jantzen, J.C., Representations of Algebraic Groups, 2nd edition, Mathematical Surveys and Monographs, 107, American Mathematical Society, Providence, RI. 2003. MR2015057 (2004h:20061)

[17] Jimbo, M., A q-analogue of $U(g l(n+1))$, Hecke algebra, and the Yang-Baxter equation, Lett. Math. Phys. 11 (1986), 247-252. MR841713 (87k:17011)

[18] Jimbo, M., Misra, K.C., Miwa, T., Okado, M., Combinatorics of representations of $U q(\widehat{\operatorname{sl}}(n))$ at $q=0$, Comm. Math. Phys. 136 (1991), 543-566. MR1099695 (93a:17015)

[19] Kac, V. Infinite dimensional Lie algebras, 3rd edition, Cambridge Univ. Press, Cambridge, 1990. MR 1104219 (92k:17038)

[20] Kashiwara, M., Schapira, P., Sheaves on manifolds, Springer, 2006. MR.1299726 (95g:58222)

[21] Kleshchev, A., Linear and projective representations of symmetric groups, Cambridge Tracts in Mathematics, Cambridge University Press, Cambridge, 2005. MR2165457 (2007b:20022)

[22] Kirillov, A.N., Reshetikhin, N. $q$-Weyl group and a multiplicative formula for universal R-matrices, Comm. Math. Phys. 134 (1990), 421-431. MR1081014 (92c:17023)

[23] Kumar, S. Kac-Moody groups, their flag varieties and representation theory, Birkhäuser, 2002. MR $1923198(2003 \mathrm{k}: 22022)$

[24] Lyle, S., Mathas, A., Blocks of cyclotomic Hecke algebras, Adv. Math. 216 (2007), 854-878. MR:2351381 (2008m:20012)

[25] Leclerc, B., Miyachi, H., Some closed formulas for canonical bases of Fock spaces, Representation Theory 6 (2002), 290-312. MR.1927956 (2004a:17022)

[26] Lusztig, G., Introduction to quantum groups, Progress in Mathematics, 110, Birkhäuser Boston, Boston, 1993. MR1227098 (94m:17016)

[27] Macdonald, I.G. Symmetric Functions and Hall Polynomials, 2nd edition, Oxford Mathematical Monographs, Oxford Science Publications, The Clarendon Press, Oxford University Press, New York, 1995. MR1354144 (96h:05207)

[28] Miemietz, V. On representations of affine Hecke algebras of type B, Algebr. Represent. Theor. 11 (2008), 369-405. MR2417511 (2010j:20010)

[29] Nakanishi, T., Tsushiya, T., Level-Rank Duality of WZW Models in Conformal Field Theory, Comm. Math. Phys. 144 (1992), 351-372. MR1152377(93a:81181)

[30] Pressley, A., Segal, G., Loop groups, Oxford University Press, 1988. MR0900587 (88i:22049)

[31] Parshall, B., Wang, J. P., Quantum linear groups, Memoirs Amer. Math. Soc. 439 (1991). MR 1048073 (91g:16028)

[32] Rouquier, R., q-Schur algebras and complex reflection groups, Mosc. Math. J. 8 (2008), 119-158. MR2422270 (2010b:20081)

[33] Shan, P., Crystals of Fock spaces and cyclotomic rational double affine Hecke algebras, Ann. Sci. Ecole Norm. Sup. 44 (2011), 147-182. MR2760196 (2012c:20009)

[34] Suzuki, T., Double affine Hecke algebras, affine coinvariants and Kostka polynomials, C. R. Math. Acad. Sci. Paris 343 (2006), 383-386. MR2259877 (2007f:17038)

[35] Uglov, D. Canonical bases of higher-level q-deformed Fock spaces and Kazhdan-Lusztig polynomials, in "Physical combinatorics (Kyoto 1999)", 249-299, Birkhäuser, Boston, 2000. MR $1768086(2001 \mathrm{k}: 17030)$

[36] Varagnolo, M., Vasserot, E., Cyclotomic double affine Hecke algebras and affine parabolic category $\mathcal{O}$, Adv. Math. 225 (2010), 1523-1588. MR2673739

[37] Wilcox, S., Supports of representations of the rational Cherednik algebra of type A, arXiv:1012.2585.

Université Paris 7, UMR CNRS 7586, F-75013 Paris, France

E-mail address: shan@math.jussieu.fr

Université PAris 7, UMR CNRS 7586, F-75013 PARIs, France

E-mail address: vasserot@math.jussieu.fr 